\title{
Lumbar extensor training in low back pain management
}

Citation for published version (APA):

Helmhout, P. H. (2009). Lumbar extensor training in low back pain management. [Doctoral Thesis, Maastricht University]. Datawyse. https://doi.org/10.26481/dis.20091217ph

Document status and date:

Published: 01/01/2009

DOI:

10.26481/dis.20091217ph

Document Version:

Publisher's PDF, also known as Version of record

\section{Please check the document version of this publication:}

- A submitted manuscript is the version of the article upon submission and before peer-review. There can be important differences between the submitted version and the official published version of record.

People interested in the research are advised to contact the author for the final version of the publication, or visit the DOI to the publisher's website.

- The final author version and the galley proof are versions of the publication after peer review.

- The final published version features the final layout of the paper including the volume, issue and page numbers.

Link to publication

\footnotetext{
General rights rights.

- You may freely distribute the URL identifying the publication in the public portal. please follow below link for the End User Agreement:

www.umlib.nl/taverne-license

Take down policy

If you believe that this document breaches copyright please contact us at:

repository@maastrichtuniversity.nl

providing details and we will investigate your claim.
}

Copyright and moral rights for the publications made accessible in the public portal are retained by the authors and/or other copyright owners and it is a condition of accessing publications that users recognise and abide by the legal requirements associated with these

- Users may download and print one copy of any publication from the public portal for the purpose of private study or research.

- You may not further distribute the material or use it for any profit-making activity or commercial gain

If the publication is distributed under the terms of Article $25 \mathrm{fa}$ of the Dutch Copyright Act, indicated by the "Taverne" license above, 


\section{Lumbar Extensor Training in Low Back Pain Management}


The studies presented in this thesis were conducted at the Department of Training Medicine and Training Physiology of the Royal Netherlands Army, and supervised by the Department of Epidemiology and Caphri Research School of the Maastricht University.

\section{Design and Lay-out:}

Hans de Haas

Sectie Grafische Diensten OG CLAS, Koninklijke Landmacht

\section{Printed by:}

Datawyse, Maastricht

\section{ISBN:}

(C) 2009, P.H. Helmhout 


\section{Lumbar Extensor Training in Low Back Pain Management}

\section{Proefschrift}

ter verkrijging van de graad van doctor

aan de Universiteit Maastricht,

op gezag van de Rector Magnificus,

Prof.mr. G.P.M.F. Mols,

volgens het besluit van het College van Decanen,

in het openbaar te verdedigen

op donderdag 17 december 2009 om 14.00 uur

door

Pieter Herman Helmhout 


\section{Promotor:}

Prof.dr. R.A. de Bie

\section{Copromotor:}

Dr. J.B. Staal

\section{Beoordelingscommissie:}

Prof.dr. L.W. van Rhijn (voorzitter)

Mw. prof.dr. M.L. Peters

Prof.dr. H. Kuipers

Prof.dr. W. van Mechelen, Vrije Universiteit Amsterdam

Dr. H.H.C.M. Savelberg 
Voor

Ans Dalenoord en Pieter Helmhout sr. 



\section{Content}

General introduction

Comparison of a high-intensity and low-intensity lumbar extensor training program as minimal intervention treatment in low back pain: a randomized trial.

A high-intensity lumbar extensor strengthening program is little better than a lowintensity program or a waiting-list group for chronic low back pain: a randomized trial

Rationale and design of a multicenter randomized controlled trial on a 'minimal intervention' in Dutch army personnel with nonspecific low back pain [ISRCTN19334317]

Isolated lumbar extensor strengthening versus regular physiotherapy in an army working population with nonacute low back pain: a randomized controlled trial

Prognostic factors for improvement in nonspecific low back pain in an army working population: secondary analyses of three randomized clinical trials

Can a fatigue test of the isolated lumbar extensor muscles of untrained young men predict strength progression in a resistance exercise program?

Exercise therapy and low back pain. Insights and proposals to improve the design, conduct, and reporting of clinical trials.

General Discussion.

Summary

Samenvatting

Dankwoord 



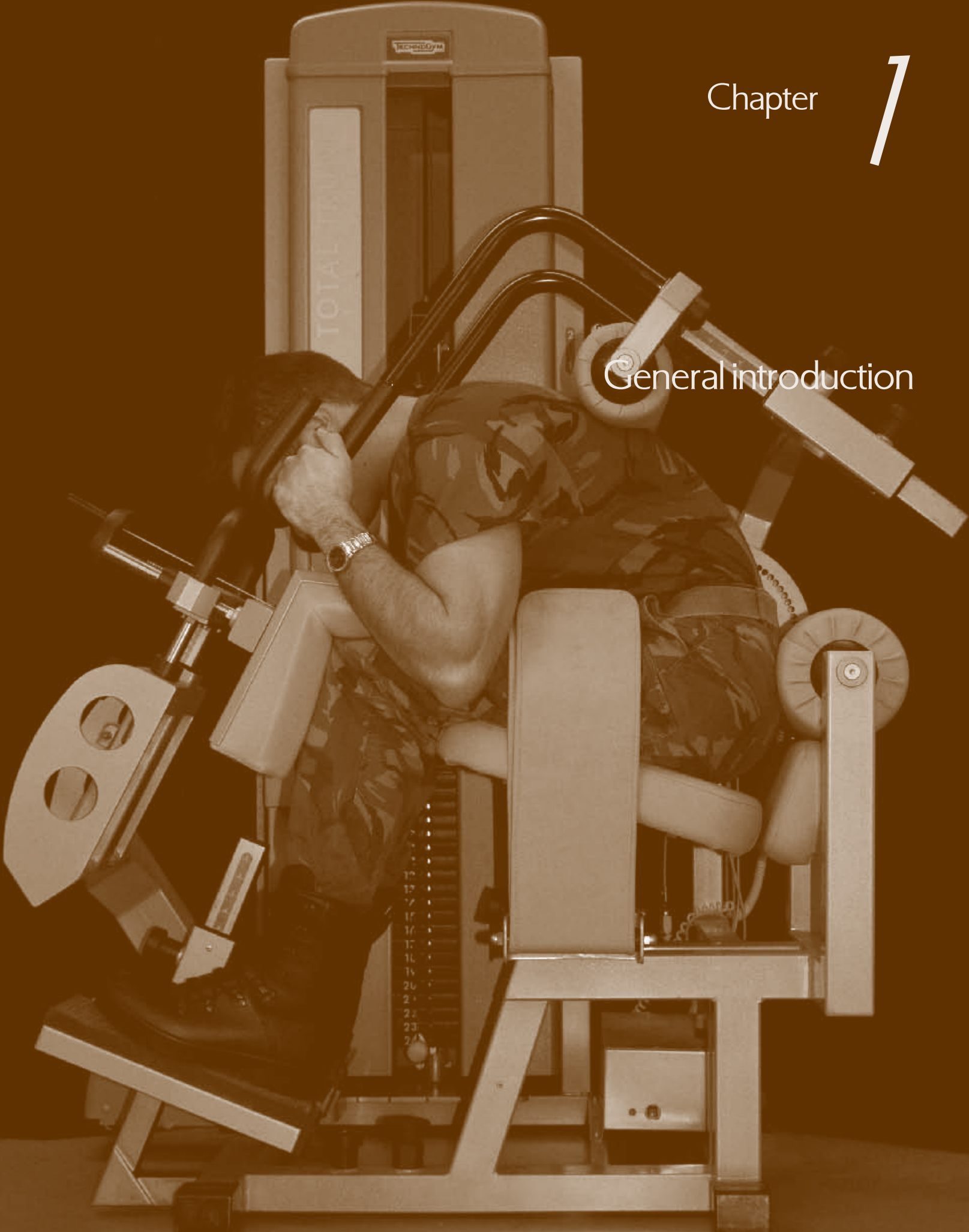




\section{Definition and aetiology of low back pain}

Low back pain (LBP) can be defined as any form of pain, muscle tension, or stiffness localized between the costal margins and the inferior gluteal folds, with or without radiation into the lower limbs. ${ }^{1}$ Any deformation of (functional-) anatomic structures of the spine may cause LBP, such as vertebrae, intervertebral discs, joints, ligaments, nerves, and muscles. The abnormality can be of mechanical origin (e.g., disc prolapse, spinal stenosis) or can have an underlying malignant or inflammatory disease (e.g., spinal tumor, ankylosing spondylitis). ${ }^{2}$ These, mostly serious, forms of spinal pathology are referred to as "specific" LBP and can cause a great personal burden for those affected.

For the majority of cases, however, no medical cause can be found for their back complaints and therefore no definitive diagnosis can be given. Labelled as "nonspecific" LBP, this group accounts for 85 to $90 \%$ of all low back pain cases. $^{3-4}$ A variety of diagnostic labels for nonspecific LBP have been applied by health care professionals, using different subgrouping schemes. ${ }^{5}$ There is no evidence to date that these diagnostic classifications can validly infer different prognoses within the nonspecific LBP population. The symptoms of nonspecific LBP may be related to mechanical strain (e.g., manual material handlings, sports activities) and/or psychosocial stressors (e.g., complex cognitive demands, job dissatisfaction), but they can also develop spontaneously. ${ }^{3}$ Moreover, more recent research has suggested a role for a genetic influence on liability to LBP. ${ }^{6}$ This thesis describes research on individuals with nonspecific LBP, unless stated otherwise.

\section{Epidemiology of low back pain}

Low back pain is a common, chronic, and recurrent condition in the general adult population of Western industrialized countries. Epidemiological studies report annual societal incidence and prevalence proportions of $19 \%$ and $45 \%$, respectively, and annual prevalence proportions in working populations ranging 
from $46 \%$ to $55 \% .^{7-9}$ Reported life-time incidences vary from $49 \%$ to $63 \%$, depending both on nationality, age or gender of the population and on the sampling method used. Lifetime prevalences widely vary from $49 \%$ to $90 \%$, with the peak prevalence occuring between ages 35 and 55.1,10

Low back pain has long been considered a benign disorder with a favourable prognosis, based on the widely accepted belief that up to $90 \%$ of LBP episodes resolve spontaneously in 3 months. ${ }^{11}$ However, more recent reviews show that LBP not always resolves itself completely when left untreated. ${ }^{12,13}$ Despite the fact that acute symptoms normally resolve in one to two months, they recur intermittently in most patients: subsequent LBP episodes range from 20-44\% within 1 year in working populations to lifetimes recurrences of up to $85 \% .{ }^{14}$ People reporting chronic LBP may have continuation of an initial episode or periodic recurrences and remissions.

Available data on the natural history, clinical course, and variation in episode duration of LBP are often incomplete and confusing, partly as a result of methodological difficulties. ${ }^{12,13,15}$ Several new definitions of a "LBP episode" have been proposed, considering pain episodes as well as intermittent pain-free periods. ${ }^{16,17}$ Throughout this thesis, nevertheless, we will use the conservative definitions of acute LBP ( $<6$ weeks of duration), sub-acute LBP (6-12 weeks of duration), and chronic LBP (> 12 weeks of duration). The main focus of this thesis is on individuals with nonacute LBP.

\section{Impact of low back pain on society, industry, health care, and the military} As said, LBP can be seen as a largely self-limiting problem, when based on the presenting complaints at a certain time point. Once back pain is showing itself as a recurrent and chronic phenomenon, it is associated with long-term disability and, consequently, a significant socioeconomic burden: some $80 \%$ of the health care and social costs are caused by $10 \%$ of persons with chronic pain and disability. ${ }^{18}$

The huge economic impact of LBP on society is mainly caused by indirect (compensation) costs due to lost work productivity and disablement, indicating 
that LBP typically affects the working population. ${ }^{1,19}$ Picavet et al ${ }^{9}$ have calculated from a cross-sectional sample of Dutch society that $50 \%$ (women) to $70 \%$ (men) of the total burden of illness of LBP problems on society - prevalences and consequences - can be addressed to the working population aged 20 to 59 years. The economic burden of chronic LBP also applies to the industry: $15 \%$ of the employees with back pain for more than 1 month are responsible for half of the work absenteeism as a result of to these complaints. ${ }^{20}$ Once chronic, the back pain condition can significantly impact an injured worker's quality of life and productivity, ${ }_{1}^{21-22}$ generating substantial costs related to medical service utilization, sick leave compensation, disability pension, and replacement costs. ${ }^{23-26}$

Back problems are among the symptoms most commonly encountered in clinical practice. Studies in Sweden and the US have reported that LBP alone accounts for 2 to $2.5 \%$ of all GP office visits, making it a top-5 reason for consulting a physician. ${ }^{27,28}$ Comparatively, data on US military populations show that back disorders are ranked second (4\%) in the age-group 17-39 and first in the agegroup 40-65 (5\%), respectively, as most frequent diagnosis at outpatient clinic visits among US Army servicemen. ${ }^{29}$ In the Royal Netherlands Army (RNLA) health care, back-related disorders represent one of the most prevalent health problems. [P. Helmhout, unpublished data, 1998] Acute LBP is the primary reason for soldiers to visit the general practitioner at a military health center. Chronic nonspecific LBP, defined as having complaints for at least 12 weeks, is one of the three most diagnosed disorders during consulting hours of Dutch military company doctors, and takes on average $15 \%$ of their weekly consulting hours time. [P. Helmhout, unpublished data, 2003]

The Royal Netherlands Army is a major professional organization in the Netherlands, employing approximately 25,000 military and civilian personnel. The diverse workforce is involved in a wide range of jobs, making the Dutch army comparable to other organizations with a high degree of physical straining job activities, such as the police force, fire brigade, health service or the construction industry. Military occupations that involve heavy physical training programs (e.g., Special Forces, Infantrymen) and/or frequent manual material handling tasks (e.g., Construction Equipment Operators, Heavy Vehicle Mechanics) are 
associated with higher-than-average risk of disability, exposing workers to occupational risk factors such as repetitive lifting, frequent bending and twisting, pushing and pulling, and awkward body postures. , $^{1,30-32}$

\section{Effectiveness of low back pain interventions}

Given the burden of LBP on society and industry, there is an urgent need for (cost-) effective therapeutic interventions specifically aimed at back complaints. Unfortunately, despite an immense investment of research in the last decades, there is disappointingly little consensus about the management of especially nonspecific LBP. A large variety of treatments exist, but none seem to be clearly superior to others. ${ }^{33-35}$

In an evidence-based review published in 2006, Van Tulder et a ${ }^{36}$ conclude that there is strong evidence that staying active and using NSAIDs and/or muscle relaxants are effective interventions for short-term pain relief in acute LBP. Advice to stay active is also effective for long-term improvement of function in acute LBP. In chronic LBP, there is strong evidence that, amongst others, NSAIDs, exercise therapy, cognitive-respondent treatment, and intensive multidisciplinary treatments are effective for short-term pain relief. NSAIDs, back schools, muscle relaxants, exercise therapy, and multidisciplinary treatments have a positive short-term effect on functional limitations. None of these interventions provide sufficient evidence for long-term effects on pain and function. Moreover, improvements are generally small.

\section{Exercise in low back pain management}

Consistently throughout the recent literature, exercise is considered an effective treatment for nonacute low back pain, at least in the short term. ${ }^{37-42}$ Maintenance of exercise benefits may require patient education and motivational strategies to reinforce behavioural change and exercise compliance..$^{41}$ One rationale behind the use of exercise in LBP management is the assumed role of decreased levels of physical activity in daily life when individuals are hindered by chronic (back) pain. The consequences of long-term inactivity have been described in terms of "Disuse Syndrome" for the general population and "Deconditioning Syndrome" for chronic LBP patients, respectively. ${ }^{43,44}$ In a review by Verbunt et $\mathrm{al}_{1}{ }^{45}$ these 
constructs and their interrelations have been modelled. Figure 1 (below) represents this model of disuse in LBP: fear of movement and/or (re)injury (avoidance behaviour) may lead to decreased levels of physical activity (disuse), which in its turns leads to physiological and functional changes (deconditioning), psychological changes, and social changes, respectively, together labelled as Disuse Syndrome. Recently, however, the validity of a deconditioning mechanism in LBP has been questioned in a review by Smeets et al. ${ }^{46}$

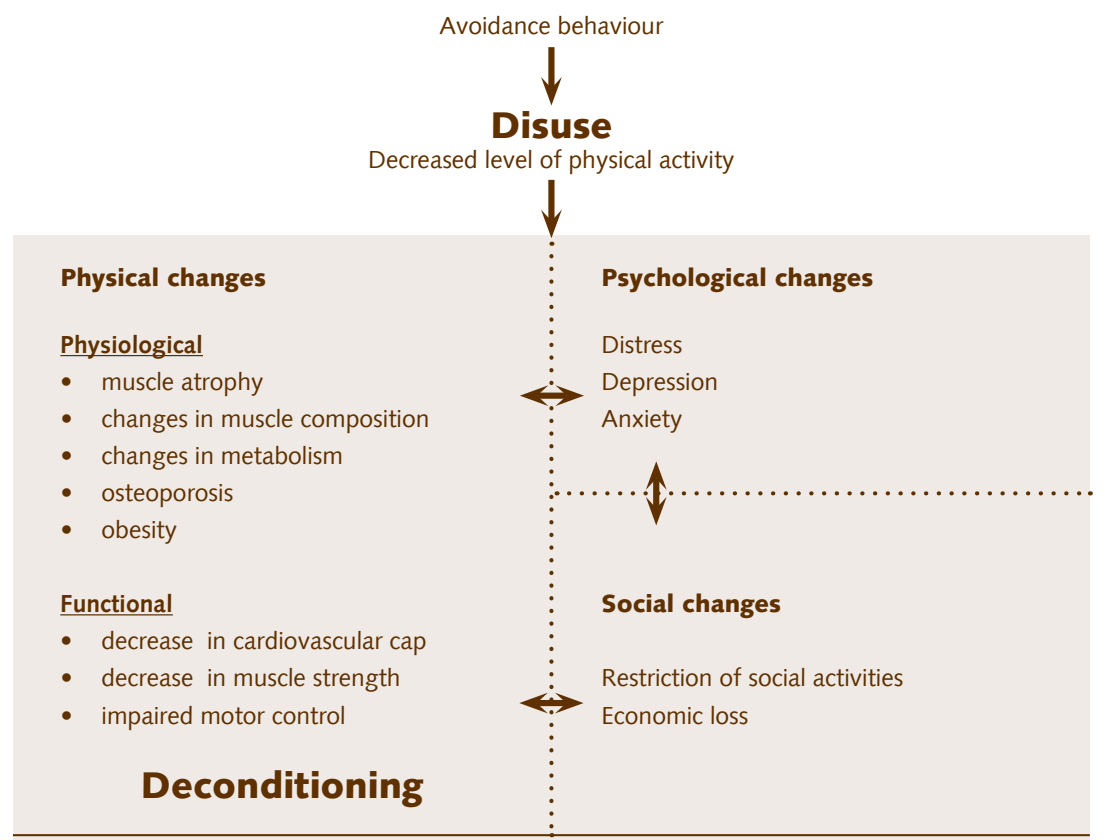

\section{Disuse Syndrome}

Figure 1. Disuse syndrome: consequences of long-term inactivity (from Verbunt et al, 2003).

For several decades, physical reconditioning by means of exercise therapy has been widely applied in clinical practice as a goal in the treatment of LBP patients. When applying exercise as a therapeutic modality, the risks as well as the benefits must be considered. In other words: does exercising a healthy or injured back increase the risk for acute back problems or further spinal injury, respectively? In a review by Rainville et al, ${ }^{40}$ several epidemiological studies have been assessed that examined the prevalence of back pain related to exercise. In summary, these studies suggest that for the general population, exercise does not increase 
the risk of back pain or sciatica and may actually have a slight protective effect against back pain. For individuals with LBP, exercise may have a positive influence on the recurrence rates of back pain and work absenteeism.

Despite the beneficial effects and relative safety of exercise, the usefulness in the treatment of chronic LBP has not gone entirely unchallenged; a number of questions regarding the method of its application and exact prescription still remain to be answered. While "exercise" is sometimes regarded as a single class of treatment, this conceptualization has failed to recognize the high variation among exercise programs and treatment protocols that have been applied in research interventions, varying widely from land-based exercise versus exercise in water to device-supported exercise versus a walking program. It is unlikely that all programs are equally effective for all patients. Reviewers have, nevertheless, routinely attempted a single summary conclusion on the efficacy of exercise, and this may contribute to the small size of its reported effects.

Several reviews $s^{37,46}$ conclude that the optimal type of exercise for chronic LBP is still unknown because the effects of specific exercises have not been systematically assessed. It is recommended that future studies should not examine interventions consisting of multiple intervention components. Instead, exercise interventions that make use of a singular concept need to be studied, whether it is strengthening specific back muscles, using mobilizing techniques, or maybe just improving general aerobic fitness aimed at reducing fear of movement.

The focus of this thesis is on one particular "unimodal" concept of exercise therapy based on sports medicine principles: isolated lumbar extensor training. In the next paragraph, we will give a summary of the rationale to use this approach in a LBP research program we have carried out in the Royal Netherlands Army (RNLA) in this last decade.

\section{Isolated lumbar extensor strengthening}

For the last ten years, RNLA clinical researchers from the Department of Training Medicine and Training Physiology have studied the potential of a sports medicine based back training approach in LBP patients. In this approach, several princi- 
ples were applied that can be derived from the rehabilitation of injured athletes. In general, return to the sports activity is the main goal of rehabilitation of sports injuries. Likewise, when managing LBP in a general population, treatment should primarily focus on a return to normal function. More specifically, early exercise is applied that is controlled, active in nature, quota-based, and progressive (sometimes referred to as "aggressive"). ${ }^{47,48}$ Progressive resistance exercise (PRE) is a method of increasing the ability of muscles to generate force. ${ }^{49}$ The principles of PRE are: (1) to perform a small number of repetitions until fatigue, (2) to allow sufficient rest between exercises for recovery, and (3) to increase the resistance as the ability to generate forces increases. These principles are detailed in the guidelines of the American College of Sports Medicine. ${ }^{50}$

In the nineties, several research groups, most of them allied to the MedX company in Florida, USA, have studied the efficacy of a specific form of progressive resistance exercise in healthy subjects and chronic back patients: isolated Lumbar Extensor Strengthening (LES). ${ }^{51-53}$ Main principle of the LES concept is that pelvic stabilization devices are used to isolate the lumbar extensor muscles and to minimize the contribution of the hip and leg muscles. ${ }^{54}$ It is hypothesized that stabilization of the pelvis is required to minimize the contribution of the (stronger) hip and leg muscles, and, consequently, to enable accurate force measurement, effective neuro-muscular education and muscular overload of the lumbar extensor muscles.

Another principle of the LES concept is that individual development of back strength is monitored with sequential isometric strength measurements of the lumbar extensors. The reason behind this choice is that, despite the fact that physical daily activities are more reflected in dynamic strength testing, isometric strength measurement is thought to allow for the identification and correction of gravity and other "nonmuscular factors", without being hampered by movement artifacts of impact and friction that appear in dynamic strength testing. ${ }^{55}$ Moreover, isometric testing accurately correlates position and torque and therefore allows reproducible measurements. ${ }^{56} \mathrm{~A}$ third principle of the LES approach is the relatively low number of training sessions that is prescribed for optimal results. Resistance training is typically described at a frequency of three times per 
week; two days of rest has been found to allow adequate recovery from exercise for most individuals beginning resistance training programs. ${ }^{50}$ However, studies on the LES concept reported significant improvements in strengths after training at a frequency of two or even one day per week, comparable to improvements after a training frequency of three times per week. ${ }^{57-59}$ It was hypothesized that the consistent, specific nature of this stimulus would create a greater amount of fatigue than was normally found with other muscle groups and that therefore a longer recovery period between training sessions was indicated.

In short, the alleged theoretical mechanism of action of LES is that it strengthens the paraspinal muscles through progressive loading, and improves the metabolic exchange of the lumbar discs through repetitive motion. ${ }^{60}$ The lumbar extensors have been considered the "weak link" in lower trunk function in healthy subjects as well as in chronic LBP patients. ${ }^{61-63}$ Through appropriate resistance training programs, reversal of these muscular dysfunctions and structural abnormalities has been documented in LBP patients..$^{53,64}$

To summarize, the "MedX studies" suggest that progressive exercise using LES is a valuable treatment for chronic LBP. Overall, promising results have been reported regarding strength improvements and pain relief in both well-trained and back-injured individuals. ${ }^{51-53,59}$ However, several methodological shortcomings hinder solid interpretation of these findings. Most encountered problems were small study population sizes, lack of randomization and inadequate or missing control groups..$^{51-53,65}$ Not surprisingly, Miltner et al ${ }^{66}$ have stressed in a review the need for randomized trials to further assess the efficacy of isolated lumbar extension exercise in LBP management. Beside methodological issues, we believed from our own experience with LES that some aspects of the described concept would need revision, to generate a truly optimal training stimulus for lumbar patients. We therefore adjusted the pelvic stabilization system for more solid and comfortable isolation of the lumbar extensor muscles, we used a different multiple isometric strength measurement procedure to better correct for nonmuscular factors, and we individualized the training load curve of each subject. [P. Helmhout, unpublished data, 2007] 


\section{Minimal exercise intervention in the RNLA}

Using this modified LES concept, we have built an exercise treatment program of 8 to 12 weeks, 1 to 2 times a week, with the training sessions taking no more than 10 to 15 minutes of specific training of the isolated lumbar extensors on modified commercially available low back training machines. This "minimal exercise intervention" has been evaluated in different RNLA population in various health care settings these last few years.

Physical fitness can be seen as a critical aspect of military preparedness and an inherent part of military service. The necessary high levels of physical fitness needed by armed forces can only be achieved through systematic, repeated, general and task-specific physical exercise. From the initial military education, soldiers are exposed to unit physical fitness training programs, including progressive resistance training on exercise machines. It can be hypothesized that this repeated work-related exposure to physical exercise shapes the beliefs, attitudes and coping behaviour of, for instance, a soldier with nonspecific back pain symptoms towards a strong physical orientation. Interventions such as our minimal exercise intervention, which predominantly focus on physical reconditioning using devices, may therefore be especially appropriate for the military population. Besides, the limited time investment per treatment session (approx. $15 \mathrm{~min}$ ) compared to usual care (approx. $30 \mathrm{~min}$ ) is expected to be highly appreciated in our military setting.

\section{Objective and research questions}

Main objective of this thesis is to assess the value of the LES concept in the management of nonacute nonspecific LBP. The two primary research questions are:

1. What is the effectiveness of a minimal intervention aimed at strengthening the (isolated) lumbar extensor muscles on nonacute nonspecific LBP in a working population, compared to a similar intervention aimed at mobilizing this muscle group and to usual care, respectively?

2. What are important prognostic factors in the 6-month development of non acute nonspecific LBP in a working population that was treated for their back using exercise therapy? 


\section{Study settings}

The research studies in this thesis were conducted within the health care service system of the Royal Netherlands Army (RNLA). First, two randomized controlled trials took place at the Department of Training Medicine and Training Physiology, Utrecht, The Netherlands, under supervision of sports physicians and health scientists. Subsequently, a multicenter trial was carried out by physiotherapists from six RNLA primary health care centers in The Netherlands and Germany, under supervision of health scientists from the Department of Training Medicine and Training Physiology.

\section{Outline of the thesis}

In Chapters 2 and 3, the results are reported of the two in-house trials on the effectiveness of the LES treatment in chronic nonspecific LBP patients, both at short-term and long-term. In both studies, LES was compared to a lower-intensity ("mobilizing") treatment program; the latter of the two studies also included a control group of subjects who were on a waiting-list for treatment. Chapter 4 describes the rationale and design of a multicenter randomized controlled trial that was performed at several RNLA military health centers, and Chapter 5 the results of this trial. The LES concept studied in the multicenter trial is slightly different from the one we used in our in-house trials, in the sense that a different test and training machine based on the same principles was used, as well as a somewhat different training protocol (10 weeks, 2 times per week versus 12 and 8 weeks, respectively, 1-2 times per week in the first trials). Chapter 6 presents secondary analyses on the merged data of the three trials, in an attempt to reveal prognostic factors for improvement in nonacute nonspecific LBP in our study population. In Chapter 7, a study is described in which the prognostic value of a three-staged fatigue test of the lumbar extensors was assessed in a healthy population of young and untrained men. Chapter 8 reflects plenary presentations and discussions of an expert panel and workshop that was held at the Amsterdam Forum VIII on Low Back Pain Research in Primary Care. Main goal of these sessions was to highlight important issues regarding the design, conduct and reporting of exercise trials and to provide suggestions for improvement in each domain. To conclude, the findings of this thesis are critically reviewed and discussed in Chapter 9. 


\section{References}

1. van Tulder M, Koes B, Bombardier C. Low back pain. Best Pract Res Clin Rheumatol 2002;16:761-75.

2. Deyo RA, Weinstein JN. Low back pain. N Engl J Med 2001;344:363-70.

3. Waddell G. The back pain revolution. London: Churchill Livingstone, 1998.

4. Deyo RA, Rainville J, Kent DL. What can the history and physical examination tell us about low back pain? JAMA 1992;268:760-5.

5. Kent $\mathrm{P}$, Keating JL. Classification in nonspecific low back pain: what methods do primary care clinicians currently use? Spine 2005;30:1433-40.

6. Hestbaek L, lachine IA, Leboeuf-Yde C, et al. Heredity of low back pain in a young population: a classical twin study. Twin Res 2004;7:16-26.

7. Jacob T. Low back pain incident episodes: a community-based study. Spine J. 2006;6:306-10.

8. Cassidy JD, Cote P, Carroll LJ, et al. Incidence and course of low back pain episodes in the general population. Spine 2005;30:2817-23.

9. Picavet HS, Schouten JS, Smit HA. Prevalence and consequences of low back problems in The Netherlands, working vs non-working population, the MORGEN-Study. Monitoring Project on Risk Factors for Chronic Disease. Public Health 1999;113:73-7.

10. Anderson WH. Perception of disease and its meanings. Lancet 1999; 354 Suppl SIV49.

11. Indahl A, Velund L, Reikeraas $O$. Good prognosis for low back pain when left untampered. A randomized clinical trial. Spine 1995;20:473-7.

12. Hestbaek L, Leboeuf-Yde C, Manniche C. Low back pain: what is the longterm course? A review of studies of general patient populations. Eur Spine J 2003;12:149 65 .

13. Pengel LH, Herbert RD, Maher CG, et al. Acute low back pain: systematic review of its prognosis. BMJ 2003;327:323.

14. Anderson GB. Epidemiological features of chronic low-back pain.Lancet 1999;354:581-5.

15. Manek NJ, MacGregor AJ. Epidemiology of back disorders: prevalencerisk factors, and prognosis. Curr Opin Rheumatol 2005;17:134-40.

16. Dionne C, Dunn KM, Croft PR, et al. A consensus approach toward the standardization of back pain definitions for use in prevalence studies. Spine 1998;33:95-103.

17. de Vet HC, Heymans MW, Dunn KM, et al. Episodes of low back pain: a proposal for uniform definitions to be used in research. Spine 2002; 27:2409-16.

18. Nachemson AL, Jonsson E. Neck and back pain: The scientific evidence of causes, diagnosis and treatment. Philadelphia: Lippincott Williams \& Wilkins, 2000

19. Dagenais $S$, Caro J, Haldeman S. A systematic review of low back pain cost of illness studies in the United States and internationally. Spine J 2008; 8:8-20.

20. Watson PJ, Main CJ, Waddell G, et al. Medically certified work loss, recurrence and costs of wage compensation for back pain: a follow-up study of the working population of Jersey. Br J Rheumatol 1998;37:82-6.

21. Morken T, Riise T, Moen B, et al. Low back pain and widespread pain predict sickness absence among industrial workers. BMC Musculoskelet Disord 2003;4:21.

22. Morken T, Riise T, Moen B, et al. Frequent musculoskeletal symptoms and reduced 
health-related quality of life among industrial workers. Occup Med (Lond) 2002; 52:91-8.

23. Lahiri S, Markkanen P, Levenstein C. The cost effectiveness of occupational health interventions: preventing occupational back pain. Am J Ind Med 2005;48:515-29.

24. Alexanderson KA, Borg KE, Hensing GK. Sickness absence with lowback, shoulder, or neck diagnoses: an 11-year follow-up regarding gender differences in sickness absence and disability pension. Work 2005;25:115-24.

25. Berkowitz SM, Feuerstein M, Lopez MS, et al. Occupational back disability in U.S. Army personnel. Mil Med 1999;164:412-8.

26. Hashemi L, Webster BS, Clancy EA, et al. Length of disability and cost of workers' compensation low back pain claims. J Occup Environ Med 1997;39:937-45.

27. Deyo RA, Mirza SK, Martin BI. Back pain prevalence and visit rates: estimates from U.S. national surveys, 2002. Spine 2006;31:2724-7.

28. Ekman L, Johnell O, Lidqren L. The economic cost of low back pain in Sweden in 2001. Acta Orthop 2005;76:275-84.

29. McDonald M, Hertz R. The health status of the United States Army. Available at: http://www.usariem.army.mil/download/usarmystatus.pdf. Accessed June 26, 2008.

30. Feuerstein M, Berkowitz SM, Peck CA, Jr. Musculoskeletal-related disability in US Army personnel: prevalence, gender, and military occupational specialties. J Occup Environ Med 1997;39:68-78.

31. Marras WS. Occupational low back disorder causation and control. Ergonomics 2000;43:880-902.

32. Burdorf A, Sorock GS, Herrick RF, et al. Advancing epidemiologic studies of occupational injury-approaches and future directions. Am J Ind Med 1997;32:180-3.

33. Ferreira ML, Ferreira PH, Latimer J, et al. Comparison of general exercise, motor control exercise and spinal manipulative therapy for chronic low back pain: A randomized trial. Pain 2007;131:31-7.

34. Cairns MC, Foster NE, Wright C. Randomized controlled trial of specific spinal stabilization exercises and conventional physiotherapy for recurrent low back pain. Spine 2006;31:E670-81.

35. Mannion AF, Muntener M, Taimela S, et al. A randomized clinical trial of three active therapies for chronic low back pain. Spine 1999;24:2435-48.

36. van Tulder MW, Koes B, Malmivaara A. Outcome of non-invasive treatment modalities on back pain: an evidence-based review. Eur Spine J 2006;15 Supp 1:S64-81.

37. Slade SC, Keating JL. Trunk-strengthening exercises for chronic low back pain: a systematic review. J Manipulative Physiol Ther 2006;29:163-73.

38. Hayden JA, van Tulder MW, Malmivaara AV, et al. Meta-analysis: exercise therapy for nonspecific low back pain. Ann Intern Med 2005;142:765-75.

39. Kool J, de Bie R, Oesch $P$, et al. Exercise reduces sick leave in patients with non-acute non-specific low back pain: a meta-analysis. J Rehabil Med 2004;36:49-62.

40. Rainville J, Hartigan C, Martinez E, et al. Exercise as a treatment for chronic low back pain. Spine J 2004;4:106-15.

41. Liddle SD, Baxter GD, Gracey JH. Exercise and chronic low back pain: what works? Pain 2004;107:176-90.

42. Mior S. Exercise in the treatment of chronic pain. Clin J Pain 2001;17:S77-85. 
43. Bortz WM. The disuse syndrome. West J Med 1984;141:691-4.

44. Mayer TG, Gatchel RJ. Functional restoration for spinal disorders: the sports medicine approach. Philadelphia, PA: Lea and Febiger; 1988.

45. Verbunt JA, Seelen HA, Vlaeyen JW, et al. Disuse and deconditioning in chronic low back pain: concepts and hypotheses on contributing mechanisms. Eur J Pain 2003;7:9-21.

46. Smeets RJ, Wade $D$, Hidding $A$, et al. The association of physical deconditioning and chronic low back pain: a hypothesis-oriented systematic review. Disabil Rehabil 2006;28:673-93.

47. Cohen I, Rainville J. Aggressive exercise as treatment for chronic low back pain. Sports Med 2002;32:75-82.

48. Lively MW. Sports medicine approach to low back pain. South Med J 2002;95:642-6.

49. Taylor NF, Dodd KJ, Damiano DL. Progressive resistance exercise and physical therapy: a summary of systematic reviews. Phys Ther 2005;85:1208-23.

50. ACSM. ACSM position stand on the recommended quantity and quality of exercise for developing and maintaining cardiorespiratory and muscular fitness, and flexibility in adults. Med Sci Sports Exerc 1998;30:975-91.

51. Leggett $S$, Mooney $V$, Matheson LN, et al. Restorative exercise for clinic lowback pain. A prospective two-center study with 1-year follow-up. Spine 1999;24:889-98.

52. Nelson BW, O'Reilly E, Miller M, et al. The clinical effects of intensive, specific exercise on chronic low back pain: a controlled study of 895 consecutive patients with 1-year follow up. Orthopedics 1995;18:971-81.

53. Risch SV, Norvell NK, Pollock ML, et al. Lumbar strengthening in chronic lowback pain patients. Physiologic and psychological benefits. Spine 1993;18:232-8.

54. Graves JE, Pollock ML, Leggett $\mathrm{SH}$, et al. Limited range-of-motion lumbar extension strength training. Med Sci Sports Exerc 1992;24:128-33.

55. Jones A. The lumbar spine. the cervical spine and the knee. Testing and Rehabilitation. MedX Corporation, Ocala, Florida/USA, 1993.

56. Shirado O, Ito T, Kaneda K, Strax T. Concentric and eccentric strength of trunk muscles: influence of test postures on strength and characteristics of patients with chronic low-back pain. Arch Phys Med Rehabil 1995;75:604-11.

57. Tucci JT, Carpenter DM, Pollock ML, et al. Effect of reduced frequency of training and detraining on lumbar extension strength. Spine 1992;17:1497-501.

58. Carpenter DM, Graves JE, Pollock ML, et al. Effect of 12 and 20 weeks of resistance training on lumbar extension torque production. Phys Ther 1991;71:580-8.

59. Graves JE, Pollock ML, Foster $D$, et al. Effect of training frequency and specificity on isometric lumbar extension strength. Spine 1990;15:504-9.

60. Mooney V, Verna J, Morris C. Clinical management of chronic, disabling lowback syndromes. In: Morris $C$, ed. Rehabilitation of the spine: a practitioner's manual. New York: McGraw-Hill, 2006.

61. Pollock $M L$, Leggett $\mathrm{SH}$, Graves JE, et al. Effect of resistance training on lumbar extension strength. Am J Sports Med 1989;17:624-9.

62. Häkkinen A, Kuukkanen $T$, Tarvainen $U$, et al. Trunk muscle strength in flexion, extension, and axial rotation in patients managed with lumbar disc herniation surgery and in healthy control subjects. Spine 2003;28:1068-73. 
63. Kankaanpaa, Taimela, Laaksonen $D$, et al. Back and hip extensor fatiga bility in chronic low back pain patients and controls. Arch Phys Med Rehabil 1998;79:412-7.

64. Parkkola R, Kujala $U$, Rytökoski $U$. Response of the trunk muscles to training assessed by magnetic resonance imaging and muscle strength. Eur J Appl Physiol Occup Physiol. 1992;65(5):383-7.

65. Taimela S, Diederich $C$, Hubsch $M$, et al. The role of physical exercise and inactivity in pain recurrence and absenteeism from work after active out patient rehabilitation for recurrent or chronic low back pain: a follow-up study. Spine 2000;25:1809-16.

66. Miltner $\mathrm{O}$, Wirtz DC, Siebert $\mathrm{CH}$. [Strengthening lumbar extensorstherapy of chronic back pain-an overview and meta-analysis]. Z Orthop Ihre Grenzgeb 2001;139:287-93. 



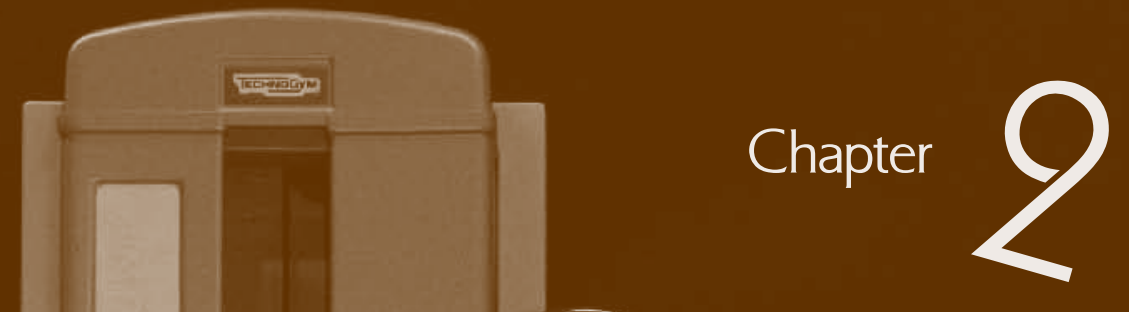

\section{low-intensity lumbar extensor training program}

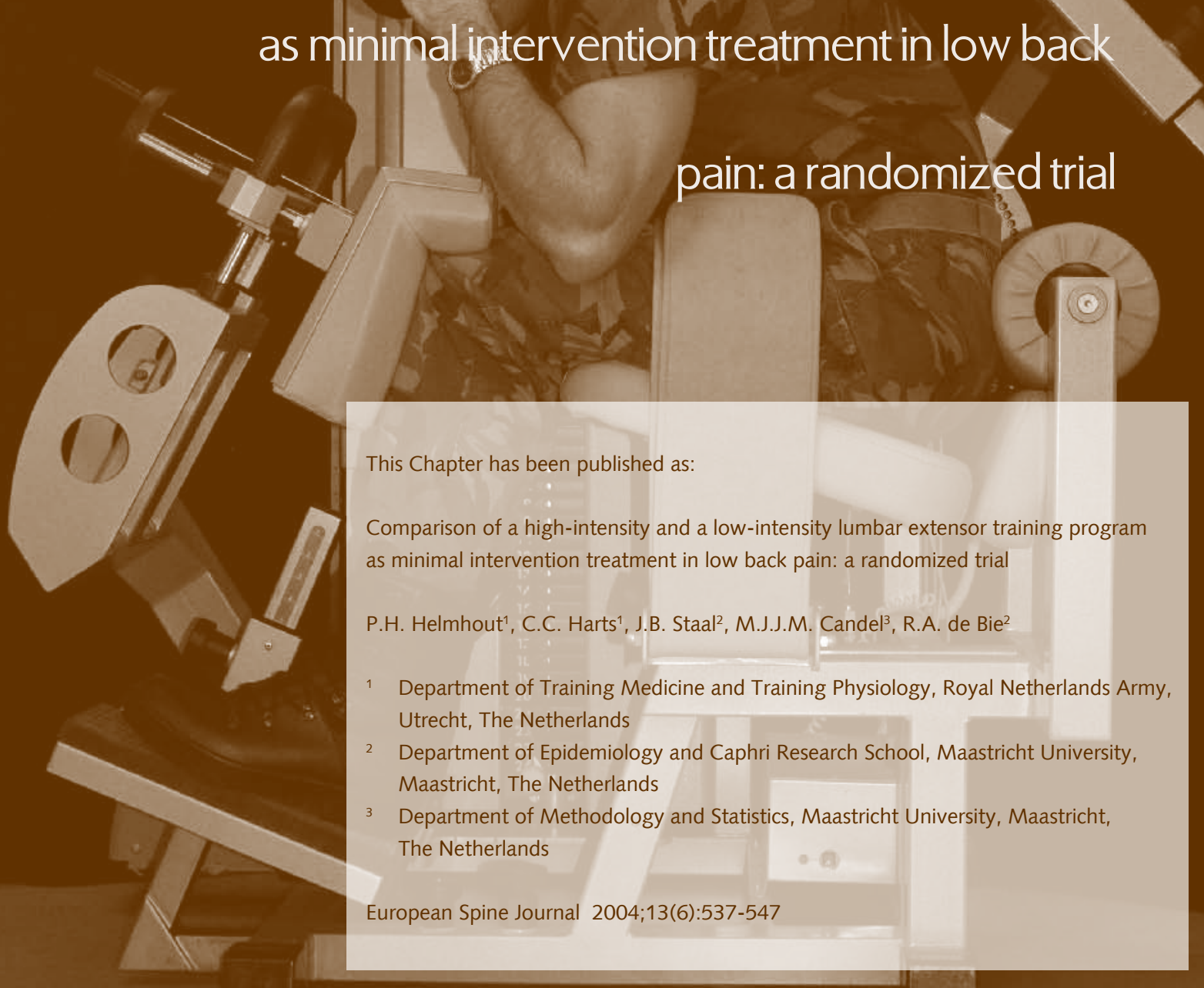




\section{Abstract}

In a randomized, observer-blinded trial, the effectiveness of a 3-month highintensity training (HIT) of the isolated lumbar extensors was compared to a low-intensity training (LIT). Eighty-one workers with nonspecific low back pain longer than 12 weeks were randomly assigned to either of the two training programs.

Training sessions were performed on a modified training device that isolated the lower back extensors. Total intervention time was limited to 5-10 minutes (one to two training sessions) per week. Training effects were assessed in terms of changes in self-rated degree of back complaints, functional disability, and general, physical and mental health. Secondary outcomes in this study were muscle strength and fear of moving the back (kinesiophobia). Outcomes were evaluated at 1,2, 3, 6, and 9 months after randomization.

The results showed that the two treatment programs led to comparable improvements in all outcome measures, except for mean isometric strength at 1, 2, 3, 6 , and 9 months and kinesiophobia score at 2 and 9 months of follow-up. The high-intensity training group showed a higher strength gain (24 to $48 \mathrm{Nm}$ ) but a smaller decline in kinesiophobia (2.5 and 3.4 points respectively), compared to the low-intensity training group.

It can be concluded that high-intensity training of the isolated back extensors was not superior to a nonprogressive, low-intensity variant in restorating back function in chronic nonspecific low back pain. In further research, emphasis should be put on identifying subgroups of patients that will have the highest success rate with either of these training approaches. 


\section{Introduction}

Physical fitness is an important precondition for military readiness. Contrary to civilian jobs, many military functions require above-average levels of physical strength and endurance to cope with daily task-related physical demands such as repetitive heavy lifting and carrying, marching or driving.

Overall, musculoskeletal injuries are by far the largest group of occupational complaints in the Royal Netherlands Army (RNLA). Low back pain (LBP) is, together with psychological overload, the most frequent diagnosed disorder during office hours of Dutch military company doctors, taking nearly $15 \%$ of their weekly office hours. These figures are expected to rise in the near future: the RNLA population is slowly ageing because of a lower recruitment rate of younger soldiers and a proposed age shift in functional dismissal, from 55 to 58 years. The risk of chronic and recurrent back pain is known to be positively correlated to age. ${ }^{1}$ Therefore, efforts to optimize the treatment of back disorders in our working population is highly valuable, both from a social and occupational perspective.

Recent systematic reviews indicate that exercise therapy is a successful approach for the restoration of chronic and recurrent LBP, at least in the short-term. ${ }^{2,3}$ However, higher quality studies generally show a lack of treatment specificity of various exercise modalities, such as aerobic exercises, strength and endurance reconditioning or mobilizing exercises. ${ }^{4-6}$ Moreover, controversy remains regarding the impact of a training stimulus, in terms of intensity, duration and frequency, on the reduction of LBP. Different explanations for this lack of specificity are given in the literature, such as nonspecific, more centrally induced training effects (e.g., a shift in pain perception) or a large heterogeneity in the chosen study populations. ${ }^{6,7}$ If, indeed, no specific dimension or type of exercise therapy is superior to another in producing optimal therapeutic outcomes, other aspects gain importance, such as costs, facilities, personnel time and capacity, treatment affinity, expectation and patient's compliance. From this perspective, back strength and endurance training in CLBP patients with the use of training devices is an interesting concept for military (and comparable) populations. 
Firstly, RNLA personnel are, from their very first initial military education, used to participate in physical exercise programs, including progressive resistance training on equipment. Second, the RNLA is well-equipped with an extensive line of modern fitness devices, including state-of-the-art lower back machines, on all major military locations throughout the country.

In recent years, we have gained experience with high-intensity, isolated training of the lumbar extensors in military personnel with LBP, using special training devices. We used a sports medicine approach, partly according to established exercise prototocols, 8,9 in which the following principles were emphasized:

- isolation of the lumbar extensors through fixation of the pelvis and thighs

- training in the individual's full range of motion

- avoiding "sticking points" - i.e., a point in the range of motion in which a relatively high resistance is experienced ${ }^{10}$ - by tuning the load curve of the weight stack to the individual's strength curve

The results we observed were satisfying to sometimes excellent in terms of pain relief and functional restoration. This was when giving them a training stimulus of not more than 5 to 10 minutes (one to two training sessions) per week.

The main objective of this study was to perform a randomized controlled trial on the relative efficacy of a high-intensity, progressive resistance training program (HIT) of the isolated lumbar extensors. We used specific training devices for a group of workers with nonspecific LBP. We compared the progressive training group with a group of workers who participated in a low-intensity, nonprogressive training program (LIT) of the same extent. Total intervention time of both "minimal intervention programs" was limited to 14 sessions of 5 to 10 minutes over a period of 12 weeks.

A multi-center study is currently underway to compare this mimimal intervention strategy with the usual treatment of LBP in the RNLA. In this paper we describe the results of the pilot-study, which evaluates the clinical importance of the treatment's progressive, muscle strengthening component. The outcome of both the HIT and LIT program is assessed in terms of changes in self-rated degree of back complaints, functional disability, and general, physical and mental health. Secondary outcomes in this study are muscle strength and fear of movement. 


\section{Methods}

\section{Study design}

We performed an observer-blinded randomized clinical trial. Measurements of outcomes were taken before randomization (baseline), at 1, 2 and 3 months after randomization (treatment period) and at 6 and 9 months after randomization (follow-up period). The study took place at the training medicine departement of the RNLA in Utrecht, The Netherlands.

\section{Study population}

The source population $(n=37,000)$ consisted of military and civilian employees of the RNLA. In the second half of 1998, all military (company) doctors and physiotherapists of the RNLA were requested to recruit participants with CLBP for our study. In addition, advertisements in military union media called for volunteers. Written informed consent was to be obtained from all participants. Further, each volunteer was given to an intake test, which consisted of history taking by a sports physiotherapist, physical examination by a sports physician and pre-assessment of the isometric back strength by a human-movement scientist. Inclusion criteria were:

- male employees of the RNLA

- more than 12 weeks of continuous or recurrent (at least 3 times a week) episodes of back pain

- pain localized between posterior iliac crests and angulus inferior scapulae

- availability to visit our department 1 to 2 times a week during 12 consecutive weeks

- willingness to abandon other treatment interventions for the lower back during the intervention period

Potential participants were excluded if they had undergone spinal surgery in the last 2 years, if they had severe back pain that was a hindrance in performing maximal isometric strength efforts, or if they had specific LBP, defined as herniated disc, ankylosing spondylitis, spondylolisthesis or other relevant neurologic diseases. 


\section{Assignment to the treatments}

All included participants were randomly assigned to a high-intensity training group (HIT) or a low-intensity training group (LIT). Randomization was done by means of a computer generated table of random numbers and a restricted randomization procedure with a block size of 4 (provided by a researcher not involved in the project). Stratification was applied for baseline scores on the Roland Disability Questionnaire11, with a cut-off level of 10 points, on the suspicion that initial level of back complaints influences the individual response to the exercise program.

\section{Content of the treatments}

The HIT program consisted of a 12-week, progressive resistance training of the isolated lumbar extensor muscle groups. The program included 10 training sessions (respectively 2 days/week in week 1 to 2 and 1 day/week in week 3 to 12) and 4 isometric strength tests (in week 1, 4, 8 and 12). The initial training load was set at approximately $35 \%$ of the maximal isometric back extension strength of the participant, measured in the first week. The goal of every training session was to perform 15 to 20 repetitions (week 1 to 2 ) or 10 to 15 repetitions (week 3 to 12 ) on the lower back machine, equivalent to approximately $50 \%$ and $70 \%$ of the one-repetition maximum ( $1 \mathrm{RM})$, respectively. If the participant was able to perform a higher number of repetitions, a $2.5 \mathrm{~kg}$ weight was added in the next training session. On the other hand, if the participant was unable to perform the minimal number of repetitions, the subsequent training load was lowered by 2.5 kilograms. This training protocol is partly based on existing protocols, 8,9 and partly on our own experiences. A comprehensive training protocol can be obtained from the authors.

In the LIT program a nonprogressive, low-intensity resistance protocol was used. Throughout the complete training period, the training load was set no higher than $20 \%$ of the maximal isometric strength, as measured in the first week. The goal of every training session was to perform 15 ( $1^{\text {st }}$ and $2^{\text {nd }}$ week after each test) or 20 repetitions ( $3^{\text {rd }}$ and $4^{\text {th }}$ week after each test) on the lower back machine. The lowest training load on the machine was a fixed weight of 2.5 kilograms. To our opinion, resistance training on this level would not generate a significant physiological strength training stimulus. 
In both exercise programs, training sessions were carried out on a standard lower back machine, used in most military sports gyms (Lower Back ROM, Technogym, Italy). For the purpose of this study, we modified the machine by applying a knee-lock system and a thigh-restraining belt. With these adjustments, hips and thighs could both be immobilized, thus allowing the participant only to move the isolated lower back. In addition, the original oval-shaped "cam" (i.e., weight stack transfer), which applied an increasing load on the back towards extension, was replaced by a circular cam. In a pre-study we found that a circular shape better reflects the optimal load curve of healthy subjects (A. Breet, internal communication, 1998). See Figure 1.
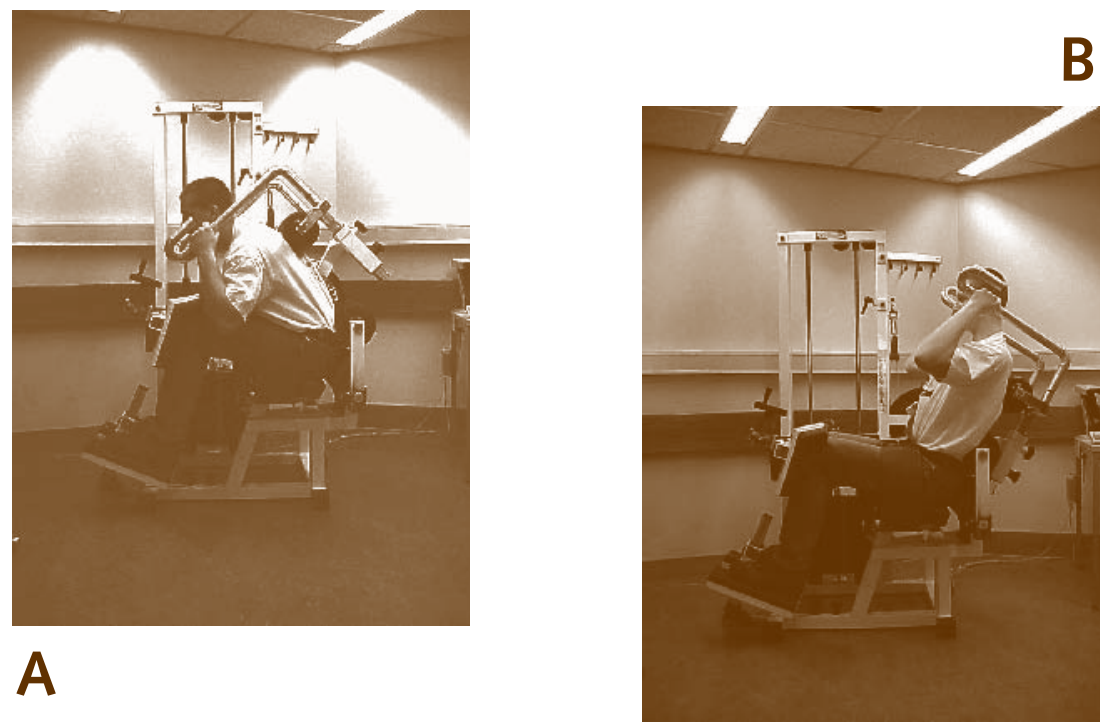

Figure 1. Modified lower back test and training machine, with subject in flexed position (A) and in extended position (B).

All training sessions in both programs were conducted by a trained physiotherapist (C.H.), who paid special attention to the execution of the training in terms of pace and movement. The flexion and extension of the lower back had to be executed in the participant's full range of motion. Movements had to be slow and controlled: moving in $2 \mathrm{~s}$ from maximal flexion to maximal extension (lifting the weight), and returning from maximal extension to maximal flexion in $4 \mathrm{~s}$ (lowering the weight). During this movement, emphasis was put on the hollowing 
and flattening of the lumbar lordosis. Every training session was preceded by a 5-minute all-body warm-up on a arm/leg ergometer (Schwinn Airdyne Pro, Balans, Nieuwegein, The Netherlands). The weight load used and the number of repetitions during each training session were recorded.

\section{Outcome measures}

\section{Self-assessed outcome measures}

At the end of the treatment period and during follow-up, participants were asked to report the degree to which their back complaints had changed since the start of the program, expressed in percentages ofincrease or decrease. In addition, various aspects of back complaints were evaluated before and after treatment and during follow-up by means of the following set of validated questionnaires:

- Roland Disability Questionnaire (RDQ): a 24-item scale, ranging from 0 to 24 points, reflecting functional disability because of LBP. ${ }^{11} \mathrm{~A}$ high score reflects a high disability rate;

- Oswestry Questionnaire (OSW): a 10-item scale, ranging from 0 to $100 \%$, to obtain a score for functional disability caused by LBP. ${ }^{12} \mathrm{~A}$ high score reflects a high rate of pain-indicated limitations; this scale is considered to be complementary to the RDQ, indicated as more sensitive to severe disability; ${ }^{13}$

- Tampa Scale for Kinesiophobia (TAMPA): a 17-item scale, ranging from 4 to 68 points, to obtain a score for the extent to which a chronic back patient fears physical damage as a result of movement, ${ }^{14,15}$ a high score reflects a high rate of fear of movement;

- MOS 36-item Short Form Health Survey (SF-36); a 36-item scale, ranging from 0 to $100 \%$, to score self-experienced health related to quality of life; ${ }^{16}$ items are clustered into eight domains from which an overall summary score, a physical component score and a mental component score can be derived. A high score on the scales reflects a high rate of self-experienced health. ${ }^{17}$

\section{Physical outcome measures}

Individual back-extension strength progression was evaluated before and after treatment and during follow-up, using repeated isometric measurements. The same modified lower back machine was used in the measurements as in the 
training exercises. To ensure valid and reliable measurements, we made several additional modifications: arm supports were attached to the arm of the machine, so that the position of the arms could be standardized in all tests. A lengthwise adjustable cable system could be attached to the machine through a notch system, after disconnection of the original weight stack-cable system. This allowed locking the arm of the machine at several angles, forwards and backwards. The force on the new system - the translation of the force on the machine arm (backward pushing or forward hanging) - was conveyed through a force sensor in the cable ( $99 \%$ accuracy up to $5 \mathrm{kN}$ ) to a personal computer, via a transducer (Digimax RS 232, Interface Mechatronic, Germany). Forces were expressed in torques $(\mathrm{Nm})$, multiplying by the radius of the circular cam $(0.12 \mathrm{~m})$. A homemade software program graphically showed the measured force signals.

Participants underwent two different measurements: a passive test and an active test. A passive test was conducted to correct for the nonmuscular torque (nonMT). The nonMT is a combination of factors that, together with the torque of the lumbar extensors, provide the gross force signal. It consists of the upper body weight, dead weight of the machine arm and the stored energy of compressed and stressed tissue in the flexed or extended torso. Correction for these factors is necessary to determine the true strength of the back extensors, the so-called net muscular torque (netMT). NonMT was measured in five positions of the machine arm: $55^{\circ}$ (full flexion), $40^{\circ}, 25^{\circ}, 10^{\circ}$ and $-5^{\circ}$ (full extension) relative to the vertical. During the passive test, participants were asked to sit as relaxed as possible in each position. In the three positions in front of the center of gravity of the torso $\left(55^{\circ}\right.$ to $\left.25^{\circ}\right)$, the arm supports could be used to hang on the fixed machine arm. If the participant was unable to reach one of the exterior positions $\left(55^{\circ}\right.$ or $\left.-5^{\circ}\right)$ because of pain or immobility, the measurement in this position was omitted. Every measurement took $6 \mathrm{~s}$, of which the last $2 \mathrm{~s}$ were converted into a mean value of nonMT (in $\mathrm{Nm}$ ).

An active test was carried out immediately after the passive test, to evaluate the maximal strength of the lumbar extensors in the same five positions. The machine arm was fixed at each angle and participants had to exert maximal backward force into the arm pad. The gross force measured was converted into 
a torque: the functional torque (FT). The netMT could then be calculated by subtracting the nonMT from the FT at every angle. A well-defined protocol was used the conduct the active test: a nonmaximal trial in each position, followed by a maximal effort for $6 \mathrm{~s}$ ( $2 \mathrm{~s}$ to smoothly build up force, $2 \mathrm{~s}$ to maintain maximal force and $2 \mathrm{~s}$ to relax slowly). Force values between the $2^{\text {nd }}$ and $4^{\text {th }} \mathrm{s}$ were converted into a mean value of netMT (in Nm).

A previous test-retest reliability study showed that the reproducibility of the NetMT was lower between an initial and second test, than between a second and third test. Based on these results, we decided to do a pre-test at the intake.

All passive and active tests were conducted by two trained test leaders, each testing a different but equally distributed group of participants from both treatment groups. Participants were verbally encouraged in every test, but no visual feedback of test performance was given. At intake, the positioning of the participant into the lower back machine was assessed based on his anthropometrics. Subjects were instructed not to exercise within 24 hours before testing. A complete, written test protocol can be obtained from the authors.

\section{Follow-up period}

Participants who completed the intervention program received questionnaires and strength measurements at 6 and 9 months after randomization, respectively. In addition, participants were asked to indicate whether they had received any treatment(s) for their LBP during this follow-up period.

\section{Blinding}

The present study is an observer-blinded trial. The training sessions and the collection of data (both from questionnaires and from strength measurements) were conducted by different persons. The two test leaders were not aware of the allocation of individual participants to one of the two groups. Conversely, the therapist who conducted the training sessions was not informed about participant scores on the strength tests; only relevant information about the measurements was given (i.e., the initial maximal isometric strength for the determination of the initial training load and the positioning into the machine). 
The two intervention programs were presented to the participants as potentially equally effective intervention modalities for the lower back while targeting different aspects: strength in the HIT group versus mobility in the LIT group. Training and test sessions of both groups were planned at separate times during the week, to prevent participants from one group exchanging treatment experiences with the other.

\section{Analysis}

All data were analysed with SPSS 11.0.1 (SPSS, 2001) or MlwiN. ${ }^{18}$ Checks for missing and incorrect values and for normality were conducted. Scores of selfassessed degree of back complaints were broken down into "improved" (20\% or more improvement) versus "not improved" (less than 20\% improvement). We chose a cut-off point of $20 \%$, which in our belief reflects a clinical relevant change in the health status of a participant.

Analyses were done using all randomized participants who provided any postbaseline data. Demographic and clinical characteristics, as well as baseline outcome measures, were summarized by descriptive statistics. Multiple Linear Regression statistics was used to assess baseline-adjusted between-group differences in all but one outcome measures at 1, 2, 3, 6, and 9 months after randomization, respectively. Chi-Square tests were used otherwise. According to the criteria suggested by Pocock et al, ${ }^{19}$ baseline covariates (characteristics and other outcomes) were entered into the regression model if related to the outcome variable $(r>0.5)$.

Mean strength development in participants who had a complete 9-month follow-up were displayed for both treatment groups. Multilevel analysis (MlwiN) was used to analyze possible trend differences between HIT and LIT in mean isometric strength across time, simultaneously correcting for baseline differeces in mean strength. All tests were judged at a two-sided significance level of $\alpha=0.05$. 


\section{Results}

\section{Patient flow and treatment compliance}

Our recruitment efforts yielded 88 employees who visited our department in Utrecht. A study population of 81 participants with LBP was selected, based on our in- and exclusion criteria. After signing informed consent, participants were randomly assigned to the HIT group $(n=41)$ or to the LIT group $(n=40)$.

Figure 2 shows a flow chart of participants and withdrawals. The HIT group showed a higher treatment compliance than the LIT group: 29 participants (71\%) in the HIT group, versus 19 participants (48\%) in the LIT group, attended all 14 training sessions. Ten participants, (24\%) versus 13 participants (33\%), missed one session; two participants ( $5 \%$ ) versus 8 participants (20\%) missed two to four sessions.

In both groups, participants reported no co-interventions during the 12-week treatment period. Four participants (12\%) in the HIT group and 8 participants $(28 \%)$ in the LIT group reported no back training and/or treatment during the follow-up period (months 3-9). Twenty-four participants (73\%) in the HIT group and 14 participants (48\%) in the LIT group participated in different kinds of exercise training, including on our specific device; five participants (15\%) in the HIT group and 7 participants (24\%) in the LIT group seeked professional help for their back in this period.

\section{Baseline characteristics and outcomes}

Table 1 shows demographic properties of the population as well as baseline values for the outcome measures. Despite our randomization strategy, the HIT and LIT group showed small differences on several baseline values. The low-compliance group (missing two to four training sessions) showed considerably worse scores on the baseline RDQ, Oswestry and SF-36 scores, than the medium and good compliers.

Table 2 shows the post-treatment minus pre-treatment scores of the outcomes at $1,2,3,6$, and 9 months after randomization. No significant between-group 
88 Workers examined for eligbility

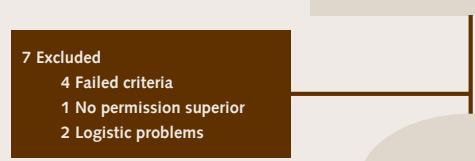

81 Randomized

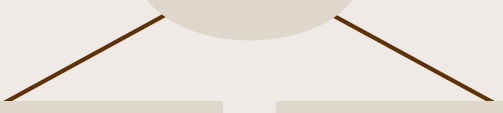

41 Assigned to High-Intensive Training group

40 Assigned to Low-Intensive Training group

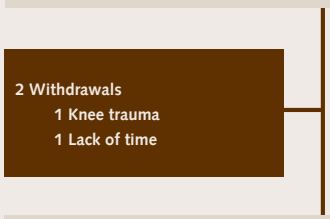

39 Complete 3-month follow-up

36 Complete 3-month follow-up

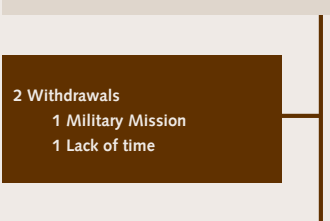

37 Complete 6-month follow-up

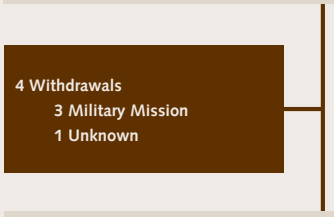

33 Complete 9-month follow-up
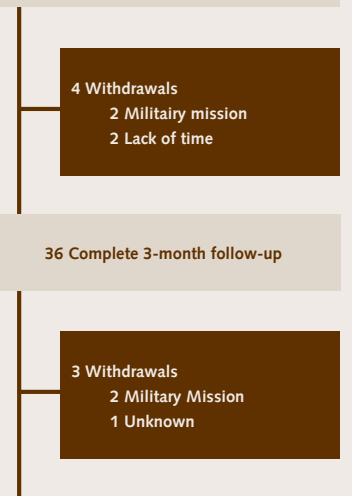

33 Complete 6-month follow-up

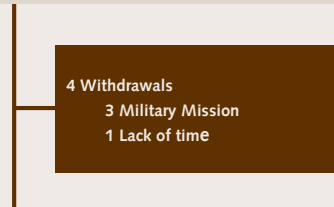

29 Complete 9-month follow-up

Figure 2. Flow chart of participants and withdrawals (months of follow-up counted after randomization).

differences in outcomes were found, except for mean isometric strength on all test moments (HIT scored 24-58 Nm higher than LIT) and for TSK score at 2 and 9 monthts (LIT scored 2.5 and 3.4 points, respectively, lower than HIT). Moreover, there was a tendency towards a significant difference in the percentage of self-assessed improvement between both groups at 3 months, the HIT group scoring $12 \%$ higher than the LIT group. 
Table 1. Demographic and clinical characteristics and baseline outcome measures of trial participants in both treatment groups, participants with medium and low compliance, and the withdrawals at the intake. Values are presented in number of participants, unless otherwise stated.

\begin{tabular}{|c|c|c|c|c|c|}
\hline & $\begin{array}{l}\text { HIT } \\
(n=41)^{a}\end{array}$ & $\begin{array}{l}\text { LIT } \\
(n=41)^{a}\end{array}$ & $\begin{array}{l}\text { Withdrawals } \\
(n=6)\end{array}$ & $\begin{array}{l}\text { Medium } \\
\text { Compliance } \\
(n=29)^{b}\end{array}$ & $\begin{array}{l}\text { Low } \\
\text { Compliance } \\
(\mathrm{n}=29)^{\mathrm{c}}\end{array}$ \\
\hline \multicolumn{6}{|c|}{ Demographic Characteristics } \\
\hline $\begin{array}{l}\text { Mean age, in years (SD) } \\
\text { Range of age, in years } \\
\text { Physically active, in } \%^{d} \\
\text { Militairy: civil employees } \\
\text { Recruited: refered patients }\end{array}$ & $\begin{array}{l}41(10) \\
21-55 \\
31(76) \\
36: 5 \\
36: 5\end{array}$ & $\begin{array}{l}40(9) \\
22-55 \\
22(55) \\
36: 4 \\
37: 3\end{array}$ & $\begin{array}{l}34(13) \\
21-50 \\
4(67) \\
5: 1 \\
3: 3\end{array}$ & $\begin{array}{l}41(11) \\
22-55 \\
16(55) \\
24: 5 \\
24: 5\end{array}$ & $\begin{array}{l}38(12) \\
22-55 \\
4(50) \\
6: 2 \\
5: 3\end{array}$ \\
\hline \multicolumn{6}{|l|}{ Clinical Characteristics } \\
\hline $\begin{array}{l}\text { Time since first LBP episode: } \\
\text { 3-6 months } \\
\text { 6-12 months } \\
1-5 \text { years } \\
\geq 5 \text { years }\end{array}$ & $\begin{array}{r}0 \\
5 \\
16 \\
19\end{array}$ & $\begin{array}{r}3 \\
7 \\
14 \\
16\end{array}$ & $\begin{array}{l}- \\
- \\
-\end{array}$ & $\begin{array}{l}- \\
- \\
-\end{array}$ & $\begin{array}{l}- \\
- \\
- \\
-\end{array}$ \\
\hline Radiation $^{\mathrm{e}}$ & 14 & 15 & - & - & - \\
\hline $\begin{array}{l}\text { Earlier therapies: } \\
\text { physical therapy } \\
\text { physiotherapy } \\
\text { manual therapy } \\
\text { orthopaedic specialist } \\
\text { no therapy }\end{array}$ & $\begin{array}{l}13 \\
15 \\
11 \\
9 \\
5\end{array}$ & $\begin{array}{r}16 \\
24 \\
8 \\
6 \\
2\end{array}$ & $\begin{array}{l}- \\
- \\
- \\
-\end{array}$ & $\begin{array}{l}- \\
- \\
- \\
-\end{array}$ & $\begin{array}{l}- \\
- \\
- \\
-\end{array}$ \\
\hline $\begin{array}{l}\text { Combination of earlier therapi } \\
1 \text { therapy } \\
2 \text { therapies } \\
\geq 3 \text { therapies }\end{array}$ & $\begin{array}{r}21 \\
11 \\
2\end{array}$ & $\begin{array}{r}12 \\
19 \\
2\end{array}$ & $\begin{array}{l}- \\
- \\
-\end{array}$ & $\begin{array}{l}- \\
- \\
-\end{array}$ & $\begin{array}{l}- \\
- \\
-\end{array}$ \\
\hline Change of work due to LBP & 4 & 4 & - & - & - \\
\hline Work absenteism due to LBP & 11 & 14 & - & - & - \\
\hline $\begin{array}{l}\text { Frequency of work absenteism } \\
\qquad \begin{array}{l}<1 \text { week in last year } \\
1-3 \text { weeks in last year } \\
\geq 3 \text { weeks in last year }\end{array}\end{array}$ & $\begin{array}{r}4 \\
29 \\
8\end{array}$ & $\begin{array}{r}3 \\
17 \\
20\end{array}$ & $\begin{array}{l}- \\
- \\
-\end{array}$ & $\begin{array}{l}- \\
- \\
-\end{array}$ & $\begin{array}{l}- \\
- \\
-\end{array}$ \\
\hline $\begin{array}{l}\text { Type of work: } \\
\text { sedentary } \\
\text { physical without } M M H \\
\text { physical with } M M H\end{array}$ & $\begin{array}{l}24 \\
6 \\
5\end{array}$ & $\begin{array}{r}25 \\
8 \\
4\end{array}$ & $\begin{array}{l}- \\
- \\
-\end{array}$ & $\begin{array}{l}- \\
- \\
-\end{array}$ & $\begin{array}{l}- \\
- \\
-\end{array}$ \\
\hline
\end{tabular}


Table 1. (Cont'd).

\begin{tabular}{|c|c|c|c|c|c|c|c|c|c|}
\hline & $\begin{array}{l}\text { HIT } \\
(n=41)^{a}\end{array}$ & \multicolumn{2}{|c|}{$\begin{array}{l}\text { LIT } \\
(n=41)^{a}\end{array}$} & \multicolumn{2}{|c|}{$\begin{array}{l}\text { Withdrawals } \\
(n=6)\end{array}$} & \multicolumn{2}{|c|}{$\begin{array}{l}\text { Mediums } \\
\text { Compliance } \\
(n=29)^{b}\end{array}$} & \multicolumn{2}{|c|}{$\begin{array}{l}\text { Low } \\
\text { Compliance } \\
(\mathrm{n}=29)^{\mathrm{c}}\end{array}$} \\
\hline \multicolumn{10}{|c|}{ Outcome Measures (mean values, SD)f } \\
\hline RDQ Score, range 0-24 points & $7.1 \quad(4.8)$ & 7.9 & $(4.8)$ & 7.2 & $(6.0)$ & 7.6 & $(4.3)$ & 12.1 & $(1.7)$ \\
\hline OSW Score, in \% & $13.9(8.0)$ & 17.4 & $(13.2)$ & 14.7 & $(13.2)$ & 18.6 & $(13.8)$ & 25.3 & $(4.5)$ \\
\hline $\begin{array}{l}\text { TSK Score, in range } 17-68 \text { points } \\
\text { SF- } 36 \text {, Score in } \%\end{array}$ & $37.0(6.1)$ & 40.0 & $(7.0)$ & 37.0 & (8.6) & 37.8 & (7.5) & 35.4 & $(9.1)$ \\
\hline Overall Summary Score & $71.2(14.2)$ & 66.9 & $(15.2)$ & 66.5 & $(22.4)$ & 68.3 & $(14.8)$ & 57.7 & $(12.6)$ \\
\hline Physical Component Score & $67.9(18.1)$ & 63.6 & $(20.8)$ & 60.9 & $(22.9)$ & 62.8 & $(18.1)$ & 49.4 & $(16.6)$ \\
\hline Mental Component Score & $83.9 \quad(18.2)$ & 79.4 & (19.8) & 77.7 & $(27.5)$ & 82.0 & $(18.4)$ & 71.7 & $(17.8)$ \\
\hline \multicolumn{10}{|l|}{ Strength: ${ }^{g}$} \\
\hline mean of 5 angles & $233 \quad(71)$ & 203 & (64) & 208 & $(52)$ & 228 & (75) & 212 & (86) \\
\hline $55^{\circ}$ flexion & $254 \quad(81)$ & 229 & (78) & 275 & (83) & 249 & (87) & 229 & $(112)$ \\
\hline $40^{\circ}$ flexion & 255 (77) & 214 & (72) & 229 & (50) & 234 & (81) & 204 & $(104)$ \\
\hline $25^{\circ}$ flexion & $245 \quad(72)$ & 206 & (72) & 227 & (66) & 237 & (201) & 196 & $(100)$ \\
\hline $10^{\circ}$ flexion & 212 (77) & 176 & (70) & 184 & (46) & 201 & (83) & 176 & (77) \\
\hline $5^{\circ}$ extension & 201 (76) & 163 & (80) & 132 & (96) & 200 & (83) & 166 & $(82)$ \\
\hline
\end{tabular}

Abbreviatons: $M M H$, manual materials handling (lifting loads); RDQ, Roland Disability Questionnaire; OSW, Oswestry Questionnaire; TSK, Tampa Scale for Kinesiophobia; SF-36, Short-Form-36 Health Survey.

Notes: a Participants refered by military (compony) doctors.

b Participants who missed one training session $(n=29)$.

c Participants who missed two $(n=6)$ three $(n=1)$ or four $(n=1)$ training session.

d Defined as 30 minutes or more physically active per day (walking/cycling to work, physical work activities, sports, physically active hobby's).

e Defined as pain radiation or tingling in the legs.

f From all outcomes measures, mean values (and SD) are presented.

$\mathrm{g}$ Isometric net muscular torque. 
Table 2. Between-group differences in the outcome measures at 1, 2, 3, 6, and 9 months after randomization.

\begin{tabular}{|c|c|c|c|c|c|c|c|}
\hline \multirow[t]{2}{*}{ Outcome Measures } & \multicolumn{4}{|c|}{ Mean Values (SD) } & \multirow{2}{*}{\multicolumn{2}{|c|}{$\begin{array}{l}\text { Between-Group } \\
\text { Differences } \\
(95 \% \mathrm{CI})^{\mathrm{a}}\end{array}$}} & \multirow[t]{2}{*}{ P-Value } \\
\hline & \multicolumn{2}{|l|}{ HIT } & \multicolumn{2}{|l|}{ LIT } & & & \\
\hline Improved (\%) ${ }^{\mathrm{b}}$ & & & \multirow{2}{*}{\multicolumn{2}{|c|}{14.1}} & \\
\hline 3 months & 64.1 & & \multicolumn{2}{|l|}{50.0} & & & $0.22^{c}$ \\
\hline 6 months & 78.4 & & 70.0 & & \multicolumn{2}{|c|}{8.4} & $0.41^{c}$ \\
\hline 9 months & \multicolumn{2}{|l|}{84.8} & \multicolumn{2}{|l|}{72.4} & \multicolumn{2}{|c|}{12.4} & $0.23^{c}$ \\
\hline \multicolumn{8}{|l|}{ Improvement $(\%)^{d}$} \\
\hline 3 months & 39.4 & (32.1) & 27.5 & (30.6) & 11.9 & $(-1.4$ to 25.3$)$ & $0.08^{c}$ \\
\hline 6 months & 51.1 & $(38.7)$ & 42.1 & (32.9) & 9.0 & (-8.1 to 22.8$)$ & $0.35^{c}$ \\
\hline 9 months & 63.0 & $(31.0)$ & 49.8 & $(33.2)$ & 13.2 & $(-3.1$ to 29.5$)$ & $0.11^{c}$ \\
\hline \multicolumn{8}{|l|}{ RDQ (0-24 points) } \\
\hline 1 month & 4.7 & $(4.1)$ & 6.0 & $(4.7)$ & -0.6 & $(-2.0$ to 0.9$)$ & 0.42 \\
\hline 2 months & 4.1 & $(3.5)$ & 5.7 & $(4.6)$ & -0.9 & $(-2.3$ to 0.5$)$ & 0.19 \\
\hline 3 months & 4.2 & $(4.0)$ & 5.5 & $(4.4)$ & -0.6 & $(-2.3$ to 1.1$)$ & 0.47 \\
\hline 6 months & 4.5 & $(4.5)$ & 4.3 & $(4.2)$ & 0.7 & $(-1.4$ to 2.9$)$ & 0.50 \\
\hline 9 months & 3.2 & $(4.2)$ & 3.2 & (3.3) & 0.6 & $(-1.2$ to 2.4$)$ & 0.52 \\
\hline \multicolumn{8}{|l|}{ OSW (\%) } \\
\hline 1 month & 13.2 & $(8.6)$ & 15.1 & $(13.0)$ & 0.5 & $(-2.9$ to 3.9$)$ & 0.77 \\
\hline 2 months & 11.5 & $(8.7)$ & 13.2 & $(12.2)$ & 0.7 & $(-2.3$ to 3.8$)$ & 0.63 \\
\hline 3 months & 10.8 & $(7.6)$ & 14.0 & (12.5) & -0.5 & (-3.7 to 2.8$)$ & 0.55 \\
\hline 6 months & 10.3 & $(9.5)$ & 13.6 & (13.4) & -0.4 & $(-4.8$ to 4.0$)$ & 0.87 \\
\hline 9 months & 9.0 & $(8.5)$ & 11.6 & (14.0) & 0.5 & $(-3.0$ to 4.0$)$ & 0.77 \\
\hline \multicolumn{8}{|l|}{ TSK (4-68 points) } \\
\hline 1 month & 36.5 & $(6.9)$ & 38.8 & $(7.0)$ & 0.2 & $(-2.0$ to 2.3$)$ & 0.88 \\
\hline 2 months & 36.5 & $(8.0)$ & 36.9 & (6.9) & 2.5 & (0.3 to 4.8$)$ & 0.03 \\
\hline 3 months & 35.0 & $(8.3)$ & 37.1 & $(7.3)$ & 0.6 & $(-2.1$ to 3.2$)$ & 0.24 \\
\hline 6 months & 35.0 & (8.9) & 36.5 & (6.9) & 1.2 & $(-1.9$ to 4.3$)$ & 0.44 \\
\hline 9 months & 34.9 & $(7.8)$ & 33.8 & $(6.4)$ & 3.4 & $(0.5$ to 6.4$)$ & 0.03 \\
\hline SF-36 overall (\%) & & & & & & & \\
\hline 1 month & 72.3 & (13.9) & 71.8 & (14.1) & -2.6 & $(-6.9$ to 1.7$)$ & 0.23 \\
\hline 2 months & 74.6 & $(14.1)$ & 71.5 & $(16.0)$ & -0.1 & $(-4.5$ to 4.3$)$ & 0.96 \\
\hline 3 months & 76.4 & $(14.6)$ & 73.3 & (15.9) & 0.5 & $(-4.3$ to 5.4$)$ & 0.83 \\
\hline 6 months & 76.9 & $(14.5)$ & 75.0 & (15.7) & 0.8 & $(-5.6$ to 7.2$)$ & 0.81 \\
\hline 9 months & 78.0 & $(14.4)$ & 78.7 & (14.3) & -3.1 & $(-9.3$ to 3.0$)$ & 0.31 \\
\hline SF-36 physical (\%) & & & & & & & \\
\hline 1 month & 69.3 & (18.3) & 70.8 & (18.1) & -4.1 & $(-11.2$ to 3.1$)$ & 0.26 \\
\hline 2 months & 73.6 & $(17.1)$ & 69.8 & $(22.1)$ & 1.5 & $(-4.9$ to 8.0$)$ & 0.64 \\
\hline 3 months & 76.6 & (17.9) & 73.5 & $(21.5)$ & 1.3 & $(-6.1$ to 8.7$)$ & 0.73 \\
\hline 6 months & 76.8 & $(20.0)$ & 74.1 & (25.1) & 1.9 & $(-9.0$ to 12.7$)$ & 0.73 \\
\hline 9 months & 82.6 & $(16.0)$ & 80.6 & (19.5) & 0.2 & $(-8.4$ to 8.8$)$ & 0.96 \\
\hline SF-36 mental (\%) & & & & & & & \\
\hline 1 month & 84.2 & $(18.3)$ & 82.7 & (18.6) & -2.0 & $(-7.3$ to 3.3$)$ & 0.46 \\
\hline 2 months & 86.3 & (15.9) & 81.8 & $(20.1)$ & 1.5 & $(-4.8$ to 7.7$)$ & 0.64 \\
\hline 3 months & 86.5 & $(17.5)$ & 82.5 & (17.9) & 1.9 & $(-4.5$ to 8.3$)$ & 0.56 \\
\hline 6 months & 87.1 & $(16.4)$ & 84.9 & (19.4) & 1.3 & $(-5.7$ to 8.4$)$ & 0.71 \\
\hline 9 months & 84.4 & $(19.7$ & 85.6 & (18.3) & -3.8 & $(-11.2$ to 3.6$)$ & 0.31 \\
\hline Strength $(\mathrm{Nm})^{\mathrm{e}}$ & & & & & & & \\
\hline 1 month & 242 & (75) & 202 & (66) & 40 & (14 to 66$)$ & 0.00 \\
\hline 2 months & 268 & (88) & 210 & (85) & 58 & (22 to 94 ) & 0.00 \\
\hline 3 months & 274 & (71) & 217 & (76) & 31 & (12 to 50 ) & 0.00 \\
\hline 6 months & 265 & (73) & 221 & (66) & 24 & (1 to 47 ) & 0.04 \\
\hline 9 months & 270 & (68) & 217 & (74) & 29 & (2 to 55 ) & 0.03 \\
\hline
\end{tabular}


Abbreviations: HIT, High-Intensive Training Group ( $n=39$ at 3 months, $n=37$ at 6 months, and $n=33$ at 9 months of follow-up); LIT, Low-Intensive Training Group ( $n=36$ at 3 months, $n=33$ at 6 months, and $\mathrm{n}=29$ at 9 months of follow-up); $95 \% \mathrm{Cl}, 95 \%$ confidence interval; RDQ, Roland Disability Questionnaire; OSW, Oswestry Questionnaire; TSK, Tampa Scale for Kinesiophobia; SF-36, Short-Form-36 Health Survey; Nm, Newtonmeter.

Notes: a Multiple Linear Regression statistics is used to assess baseline-adjusted between-group differences in all outcome measures except "Improved".

b Percentage participants with a self-assessed percentage decrease $(+)$ in back complaints of more than $20 \%$ compared to baseline (measured only after the training program).

c Chi-Square Test.

d Mean self-assessed percentage decrease (+) or increase (-) in back complaints compared to baseline (measured only after the training program).

e Mean isometric net muscular torque of 5 angles.

In Figure 3, back strength measurements (mean isometric strength of five angles) for both groups are displayed for all test moments in the 9-month follow-up. The trend we see in this figure has been analyzed further, employing multilevel analysis.

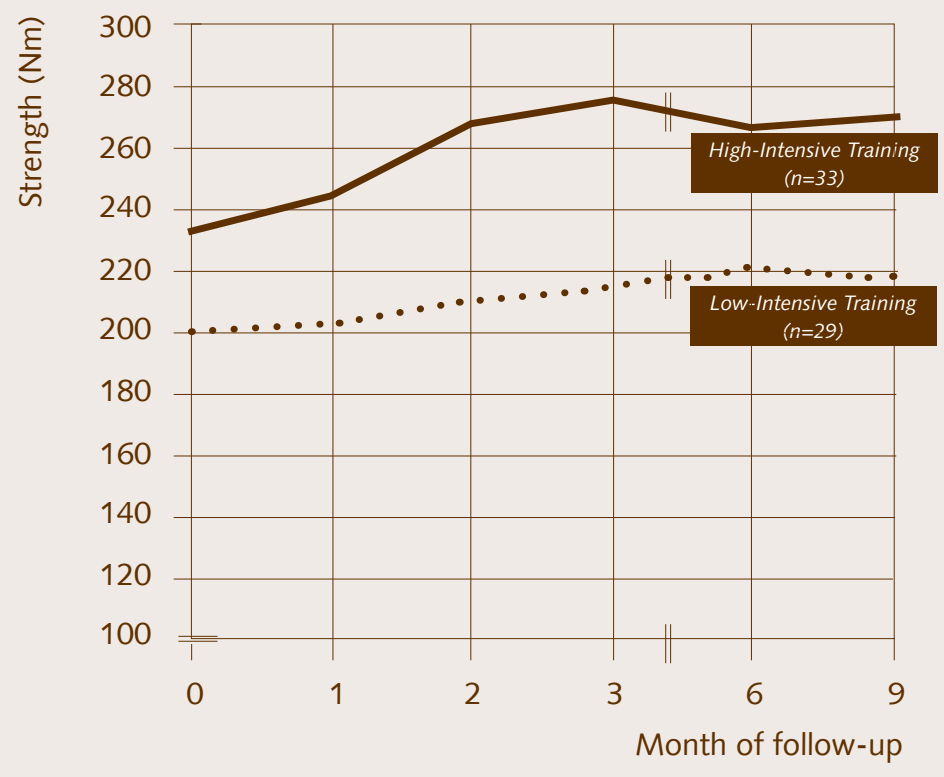

Figure 3. Strength development (mean isometric strength of 5 ang/es) in participants from both intervention groups who had a complete 9-month follow-up. 
Table 3 presents strength across time, corrected for the effects of baseline differences in strength between both groups. The results show that the difference in strength development between HIT and LIT follows a curvilinear trend, which can be described by: $11.970 * t-1.018 * t^{2}$. In the first \pm 6 months $(t=5.88)$, the group difference in strength increases until about $35 \mathrm{Nm}$ in favor of HIT, followed by a decrease. During the total period of study ( 9 months), there is a positive effect of HIT versus LIT on strength. Moreover, the effect of one unit of increase in the baseline value of strength on the strength at time $t$ can be expressed as: $0.853 * t-0.202 * t^{2}+0.013 * t^{3}$. This means that, at every test moment after randomization ( $t=1,2,3,6$, and 9$)$, the initial strength measurement has a positive effect on strength at that moment.

Table_3. Multilevel analysis for isometric back strength across time.

\begin{tabular}{|c|c|c|c|c|}
\hline Predictor & $\begin{array}{l}\text { Parameter } \\
\text { estimate }\end{array}$ & $\begin{array}{c}\text { Standard } \\
\text { Error }\end{array}$ & T-Value & P-Value \\
\hline Time $^{\mathrm{a}}$ & 136.985 & 13.365 & 10.25 & $<0.001$ \\
\hline Time $\times$ baseline strength & 0.853 & 0.049 & 17.408 & $<0.001$ \\
\hline Time $x$ group ${ }^{b}$ & 11.970 & 4.549 & 2.631 & 0.009 \\
\hline Time $^{2}$ & -95.710 & 6.428 & -14.890 & $<0.001$ \\
\hline Time $^{2} \times$ baseline strength & -0.202 & 0.015 & -13.467 & $<0.001$ \\
\hline Time $^{2} \times$ group & -1.018 & 0.467 & -2.180 & 0.032 \\
\hline Time $^{3}$ & 19.547 & 1.135 & 17.222 & $<0.001$ \\
\hline Time $^{3} \times$ baseline strength & 0.013 & 0.001 & 13 & $<0.001$ \\
\hline Time $^{4}$ & -1.168 & 0.065 & -17.969 & $<0.001$ \\
\hline \multicolumn{5}{|l|}{ Random Effects } \\
\hline variance of random slope for time & 242.736 & 62.217 & & \\
\hline variance of random slope for time ${ }^{2}$ & 1.685 & 0.668 & & \\
\hline error variance & 1272.918 & & & \\
\hline
\end{tabular}

Notes: a Test moment at 1, 2, 3, 6, and 9 months respectively (Time, Linear trend; Time², quadratic trend; Time ${ }^{3}$, cubic trend; and Time ${ }^{4}$, quartic trend).

b Treatment group: HIT =1, LIT = 0. Group effects were corrected for baseline differences in strength. 


\section{Discussion}

\section{Main findings}

In a randomized, observer-blinded trial, the effectiveness of a 12-week highintensity exercise program of the isolated lumbar extensors was compared to a low-intensity program. Our results show that the two treatment programs led to comparable improvements in all outcome measures, except for mean isometric strength at 1, 2, 3, 6, and 9 months and TSK score at 2 and 9 months of followup. The high-intensity training group showed a higher strength gain (24-58 Nm) and a smaller decline in fear of movement (2.5-3.4 points less), compared to the low-intensity training group.

\section{Generalizability of the study population}

The population of this study represents a group of male employees with LBP with, on average, long-standing, but moderate, functional disability that enables them to work with only occasional absenteeism.

Baseline scores on functional disability (RDQ and OSW) were comparable to those of other study populations with moderate severe LBP., 4,6,20 However, scores on fear of movement (TSK) ${ }^{15,21}$ and self-experienced health (SF-36) ${ }^{22,23}$ were generally higher. Compared to a Dutch population of healthy men between 25 and 55 years of age, ${ }^{23}$ our CLBP population scored 8 points lower on the SF-36 Overall Summary Score, 16 points lower on the SF-36 Physical Component Score and only 1 point lower on the SF-36 Mental component Score. Mc Horney et $\mathrm{al}^{24}$ noted that a difference of 23 points in the domain of "physical functioning" (PF) reflects the impact of a complicated chronic medical condition, while a difference of 27 points in the domain of "mental health" $(\mathrm{MH})$ is equivalent to the impact of serious depressive symptoms. Our population scored 11 points lower on $\mathrm{PF}$ and 2 points on $\mathrm{MH}$, indicating that perceived health problems were not severe and mainly focused on physical aspects.

The fact that a strictly male population was used prevents this study from possible gender-related bias, such as different morphologic adaptations of strength training in men and women.7,25 
Finally, participants in this study were partly recruited via advertisements $(n=73)$, which may have attracted a group with good self-motivation to alleviate their back problems, compared to the participants $(n=8)$ who were referred by a clinician. Nevertheless, recruitment bias is not to be expected because both groups were randomized rather evenly into each of the interventions. A comparable distribution was found in the subgroups (withdrawals, medium compliers, low compliers). We therefore do not suspect a recruitment bias.

\section{Considerations regarding the main findings}

The main goal of the current study was to compare the effectiveness of a high-intensity and low-intensity back strengthening program, both of limited dimension. We hypothesized that the progressive, high-intensity character of the intervention was a key element in the restoration of back function. Our results do not confirm this hypothesis: both training groups showed comparable improvements on all outcomes.

This is in agreement with the recent literature, in which training intensity is not seen as the crucial factor in restoring back function. In a systematic review, Van Tulder et $\mathrm{al}^{3}$ conclude that there is strong evidence ("level 1 ") that exercise strengthening exercises are not more effective than other types of exercises. This conclusion is mainly based on four high-quality trials that made use of progressive trunk and leg exercises, 5,7,26 sometimes in combination with other treatments. ${ }^{4}$ Total intervention time in these studies varied from 15 to 90 minutes, from two to three times a week, and from 4 to 54 weeks. Mannion et al ${ }^{6}$ mention a "paradox in the concept of training specificity", in which the precise mode of therapy seems of limited importance for its effectiveness. More centrally induced training effects, like modifications in pain perception and analgesic effects of exercise, are suggested to be responsible for the improvements in back function. They conclude that physical restoration of moderate CLBP using specific types of exercise seems not to be justified. ${ }^{27}$

Despite the lack of differences in outcomes, both groups did improve over time. Mainly for practical reasons and reasons of study power, we chose not to divide the total study population into three groups by including a waiting-list group. 
Consequently, we are not able to judge whether possible placebo, learning, novelty or Hawthorne effects might have lead to an overestimation of the posttreatment improvements found. Since the majority of our participants already had longstanding back pain before entering the study, the post-treatment improvements found in this study cannot simply be explained by spontaneous recovery. The continuous character of these improvements - up to half a year after the end of the exercise program - could be explained from the relative high percentage of participants (84\%) continuing some sort of back training or treatment during the follow-up period.

Analyses on strength development show that the strength-gaining effect of the HIT program exceeds the training period by approximately another 3 months. This is an interesting finding, considering the minimal intervention dimensions of the program. It might be explained by the high percentage of patients who continued to participate in exercise training in the follow-up period. Moreover, we can learn from the analysis that a higher initial back strength leads to a higher strength increase in time. From a physiological point of view, we know that strength training normally follows the law of diminishing returns, i.e., individuals with low initial strength have a higher strength development potential than individuals who are already strong at baseline. ${ }^{28}$ We do not have a solid explanation for these findings, other than speculative ones, e.g., back patients with a stronger back, possibly as a result of a more physically active background, have respectively more faith in, less fear of, and higher expectations of our back strengthening approach.

An interesting finding is the higher degree of functional disability and lower selfexperienced (physical) health at baseline among the low compliers, i.e., participants who missed two to four training sessions, compared to the medium and high compliers. It seems that the severity of complaints at least partly influences the motivation to participate in the treatment program.

\section{Limitations of the study}

The contrast between both interventions appeared to be less than expected. The training load was set at a maximum of $20 \%$ of the initial mean isometric 
strength of five angles. We observed that, for some participants in the LIT group with extreme low back strength at baseline, even a weight-stack without loads together with the upper body weight in flexed position, exceeded the $20 \%$ intensity training stimulus. These factors might have diminished the contrast between both exercise programs. The LIT group showed a somewhat lower treatment compliance than the HIT group, which in its turn may have enlarged the intervention contrast between both groups.

The number of dropouts in our study was relatively high but evenly distributed among the two training groups, as well as the reasons of withdrawal. There was no indication that treatment dropouts had different characteristics and baseline outcomes than the remaining participants.

\section{Comparison of treatment effects with references}

By using effect sizes, calculated as [post-mean - pre-mean]/pre-standard deviation, the outcome responses of our study can be compared with those of similar intervention studies in comparable study populations. We only looked at the outcome responses that showed (near) significant post-treatment differences between the HIT and LIT group: percentage self-assessed improvement, mean strength, and fear of movement.

Manniche et $\mathrm{al}^{26}$ reported $74 \%$ responders ("satisfied to very satisfied posttreatment") in a group that had followed a high-intensity back strengthening program, and $42 \%$ responders in a low-intensity group. These percentages are comparable to our results, in which "responder" is defined as $20 \%$ or more improvement in back function: $64 \%$ responders in the HIT group and $50 \%$ in the LIT group. Leggett et $\mathrm{a}^{22}$ reported a higher responder rate of $80 \%$ after a multimodal exercise program including isolated back extensor strenghtening, using a 3-level appraisal score: better, same, worse.

With respect to back strength, effect sizes for our HIT group of 0.56-0.65 (flexion-extension) at 12 weeks, were comparable to those in a study by Risch et $\mathrm{al}^{29}$ using back-isolating devices for patients with long-standing CLBP: 0.61-0.68 at 10 weeks. The effect sizes of our LIT group were somewhat higher (0.00-00.36) 
than those of their waiting-list group (0.13-0.00), which can be explained by a combination of training respons and attention bias (Hawthorne effect). In a study of Leggett et al ${ }^{22}$ with isolated back strengthening as part of a multimodal exercise intervention, higher effect sizes of 1.03 (better), 0.86 (same), and 0.65 (worse) were reported in patients from two outpatient CLBP treatment centers. The above-mentioned studies, as well as our study, used their training machine as measurement device, which presumably overestimates the effect sizes found.

To the authors knowledge, no intervention study comparable to ours has been done using the Tampa Scale for Kinesiophobia. Overall, few studies have focused on the use of specific training devices as the only intervention treatment for LBP, and no study so far has used a minimal intervention strategy comparable to ours. The magnitude of improvements in back function in the current study are in line with those reported in other studies using more extended exercise programs. This finding does not confirm a dose-respons relationship between exercise and back complaints, suggested by Bronfort et al. ${ }^{4}$ Vuori ${ }^{30}$ mentions that physiological training principles, in terms of type and dose of exercise, cannot simply be applied when other than physiological conditioning factors of physical activity are involved (e.g., individual preferences and perceptions).

\section{Conclusions}

Concordant to the literature, we were unable to demonstrate that progressive, high-intensity training of the isolated back extensors was superior to a nonprogressive, low-intensity variant in restorating back function. In terms of fear of movement, the gradual training approach even seems favorable. Nevertheless, other findings in this study indicate that some individuals with chronic LBP might benefit more from an agressive approach: our high-intensity training group showed a trend towards a higher post-treatment improvement rate, as well as a higher treatment compliance and a higher willingness to participate in physical exercise on the longer term. In future research, emphasis should be put on identifying subgroups of patients that will have the highest success rate with either of these training approaches. Clearly, larger studies with more power are needed to do such an analysis. 


\section{References}

1. Marras WS. Occupational low back disorder causation and control. Ergonomics 2000;43:880-902.

2. Mior S. Exercise in the treatment of chronic pain. Clin J Pain 2001;17:S77-85.

3. Tulder van $M$, Malmivaara $A$, Esmail $R$, et al. Exercise therapy for low back pain: A systematic review within the framework of the Cochrane Collaboration Back Review Group. Spine 2000;25:2784-96.

4. Bronfort $\mathrm{G}$, Goldsmith $\mathrm{CH}$, Nelson $\mathrm{CF}$, et al. Trunk exercise combined with spinal manipulative or NSAID therapy for chronic low back pain: A randomised, observerblinded clinical trial. J Manipulative Physiol Ther 1996;19:570-82.

5. Ljunggren $A E$, Weber $H$, Kogstad $O$, et al. Effect of exercise on sick-leave due to lowback pain. Spine 1997;22:1610-17.

6. Mannion AF, Müntener $M$, Taimela $S$, et al. A randomised clinical trial of three active therapies for chronic low back pain. Spine 1999;24:2435-48.

7. Hansen FR, Bendix T, Skov P, et al. Intensive, dynamic back-muscle exercises, conventional physiotherapy, or placebo-control treatment of low-back pain: A randomized, observer-blind trial. Spine 1993;18:98-108.

8. Graves JE, Webb DC, Pollock ML, et al. Pelvic stabilization during resistance training: Its effect on the development of lumbar extension strength. Arch Phys Med Rehabil 1994;75:210-5.

9. Pollock ML, Leggett $\mathrm{SH}$, Graves JE. Effect of resistance training on lumbar extension strength. Am J Sports Med 1989;17:624-29.

10. Darden E. The Nautilus Book., Chicago: Contemporary Books Inc., 1990.

11. Roland M, Morris R. A study of the natural history of back pain: Part I. Development of a reliable and sensitive measure of disability in low-back pain. Spine 1983;8:141-4.

12. Fairbank JC, Couper J, Davies JB, et al. The Oswestry low back pain disability questionnaire. Physiother 1980;66:271-3.

13. Beurskens $A J$, Vet $\mathrm{HC}$ de, Köke AJ, et al. Measuring the functional status of patients with low back pain. Assessment of the quality of four diseasespecific questionnaires. Spine 1995;20:101728.

14. Kori SH, Miller RP, Todd DD. Kinisiophobia: A new view of chronic pain behavior. Pain Manag 1990;Jan/Feb:35-43.

15. Vlaeyen JW, Kole-Snijders AM, Boeren RG, et al. Fear of movement/(re)injury in chronic low back pain and its relation to behavioral performance. Pain 1995;62:363-72.

16. Zee van der K, Sanderman R. [Het meten van de algemene gezondheidstoestand met de RAND-36: Een handleiding.] Groningen: Noordelijk Centrum voor Gezondheidsvraagstukken; 1993.

17. Shmueli A. The SF-36 profile and health-related quality of life: An interpretative analysis. Qual Life Res 1998;7:187-95.

18. Rasbash J, Browne W, Goldstein H, et al. A User's Guide to MlwiN. London: Institute of Education, 1999.

19. Pocock SJ, Assmann SE, Enos LE, et al. Subgroup analysis, covariate adjustment and baseline comparisons in clinical trial reporting: current practice and problems.

Statist Med 2002;21:2917-30. 
20. Kuukkanen T, Mälkiä E. Muscular performance after a 3 month progressive physical exercise program and 9 month follow-up in subjects with low back pain. A controlled study. Scand J Med Sci Sport 1996;112-21.

21. Vlaeyen JW, Kole-Snijders AM, Rotteveel AM, et al. The role of fear of movement/ (re)injury in pain disability. J Occup Rehabil 1995;5:235-52.

22. Leggett $\mathrm{SH}$, Mooney $\mathrm{V}$, Matheson LN, et al. Restorative exercise for clinical low back pain: A prospective two-center study with 1-year follow-up.

Spine 1999;24:889-98.

23. Ware J, Sherbourne C. The MOS 36 item Short Form Health Survey (SF-36). Med Care 1992;30:473-83.

24. McHorney CA, Kosinski M, Ware JE Jr. Comparisons of the costs and quality of norms for the SF-36 health survey collected by mail versus telephone interview: Results from a national survey. Med Care 1994;32:551-67.

25. Rissanen $A$, Kalimo $H$, Alaranta $H$. Effect of intensive training on the isokinetic strength and structure of the lumbar muscles in patients with chronic low back pain. Spine 1995;20:333-40.

26. Manniche $C$, Lundberg E, Christensen I, et al. Intensive dynamic back exercise for chronic low back pain: A clinical trial. Pain 1991;47:53-63.

27. Mannion AF, Junge $A$, Taimela $S$, et al. Active therapy for chronic low back pain: Part 3. Factors influencing self-rated disability and its change following therapy. Spine 2001;26:920-29.

28. Vrijens J. [Basis voor verantwoord trainen.] Gent: PVLO, 1984.

29. Risch SV, Norvell NK, Pollock ML, et al. Lumbar strengthening in chronic low back pain patients. Spine 1993;18:232-38.

30. Vuori IM. Dose-response of physical activity and low back pain, osteoar thritis, and osteoporosis. Med Sci Sports Exerc 2001;6:S551-86. 



\section{Abstract}

Question: Is eight weeks of high-intensity strengthening of the isolated lumbar extensors more effective than low-intensity strengthening or no strengthening? Are any gains maintained 16 weeks later?

Design: Randomized, three-arm trial with concealed allocation, assessor blinding, and intention-to-treat-analysis. Participants in the waiting list control group were randomized again, after the first 8 weeks, to either the high-intensity or the low-intensity strengthening program.

Participants: Sixty-five army personnel with chronic nonspecific low back pain. Intervention: The high-intensity training group received 10 sessions of 15 to 20 repetitions for the isolated lumbar extensor muscles. The low-intensity training group received a nonprogressive, low-intensity resistance protocol.

Outcome measures: Primary outcomes were global perceived effect and disability. Secondary outcome measures were health-related quality of life, fear of movement/(re-)injury and isometric lumbar extensor muscle strength. Measures were taken before and after the training and 16 weeks later.

Results: At eight weeks, SF-36 overall score was on average 7\% $(95 \% \mathrm{Cl} 1$ to 13) greater in the high-intensity training group compared to the low-intensity training group and the waiting list control group, and self-assessed decrease of back symptoms was on average 39\% (95\% Cl 14 to 64) greater in the high-intensity training group compared with the waiting list control group. There was no difference in improvement between the groups for any other outcome at 8 and 24 weeks.

Conclusions: Although some beneficial effects were found, the results of this high-intensity strengthening program of the isolated lumbar extensor muscles do not clearly support the generally-claimed beneficial influence of exercise for chronic nonspecific low back pain. 


\section{Introduction}

Nonspecific low back pain is, together with psychological overload, the disorder most frequently diagnosed during office hours of Dutch military company doctors (Royal Netherlands Army, 1998). Hence, effective intervention for this disorder as well as prevention strategies are of great importance for army personnel and in particular for soldiers with physically demanding job tasks such as repetitive lifting and carrying, marching and digging. Exercise therapy is currently the most popular treatment for low back pain prescribed by doctors and physiotherapists of the Royal Netherlands Army for low back pain. An advantage of this treatment approach is that it matches well with the relative active lifestyle habits of many soldiers.

Previous research has indicated that exercise therapy is beneficial for the treatment of chronic and recurrent low back pain. ${ }^{1}$ Specific dynamic extension training of the lumbar back muscles is the exercise therapy commonly applied at our department in cases of subacute and chronic low back pain ${ }^{1}$. We thereby aim at increasing the cross-sectional area, strength, and endurance of the back extensor muscles, improvement of co-ordination, and reduction of fear of movement in order to improve disability. Randomized prospective studies have shown that dynamic extension exercises may indeed cause physiological effects with regard to strength and endurance..$^{2-4}$ In a comprehensive Finnish cohort study consisting of 535 subjects, Rissanen et $\mathrm{al}^{2}$ also showed that poor dynamic trunk extension performance was associated with back-related permanent work disability. The working mechanisms of dynamic extension exercises do not merely involve physiological adaptations, but probably also psychological phenomena such as reduction in psychological distress, fear-avoidance beliefs, and fear of movement. 5,6 The supposed benefits of dynamic extension exercises, however, need firmer scientific confirmation in randomized controlled trials which also address the optimal intensity of the dynamic extension exercises for low back pain.

In order to investigate the effectiveness of our dynamic extension exercise programs, we conducted a randomized controlled trial among Royal Netherlands Army personnel with chronic low back pain. 
Our research questions were:

1. Is eight weeks of high-intensity strengthening of the isolated lumbar extensors more effective than low-intensity strengthening or no strengthening (i.e., waiting list control)?

2. Are any gains maintained 16 weeks after the cessation of the intervention?

\section{Methods}

\section{Design}

A three-arm observer-blinded randomized controlled trial was carried out among employees with chronic low back pain in the Royal Netherlands Army. The source population $(n=37,000)$ was informed about the planned study in the second half of 1998 by advertisements in military union media calling for volunteers to participate. After the informed consent procedure, all volunteers had their history taking by a physiotherapist, were physically examined by a sports physician, and had their isometric back strength measured by a human movement scientist. Subsequently, eligible participants were randomly assigned to the high intensity training-group, the low intensity training-group or the waiting list control group. Concealed randomization was performed by means of a computer-generated table of random numbers with a block size of 6 . A researcher who was not involved in the randomization procedure or measurement procedure provided the table of random numbers. Eight weeks after the initial randomization, the participants in the waiting list group were randomized again, using the same concealment, to either the high intensity training-program or the low intensity training-program.

Outcomes for the high-intensity training and low-intensity training groups were measured before randomization (baseline), at 8 weeks (end of intervention), and at 24 weeks after randomization (follow-up). For the waiting list-group, outcomes were measured before the first randomization (baseline), at 8 weeks (waiting list period), at 16 weeks (end of intervention), and at 32 weeks after the first randomization (follow-up). Measurement of outcomes was carried out by the principle investigator or research assistants who were not aware of the group allocation. Both intervention and measurements were carried out at 
the Department of Training Medicine and Training Physiology in Utrecht, the Netherlands. The study protocol was reviewed and ethically approved by the inspectorate of the army medical services.

\section{Participants}

The inclusion criteria specified that participants should: be male employees of the Royal Netherlands Army in the age of 18-54 years; have experienced low back pain for more than 12 weeks; be available to visit our department 1 to 2 times a week during 8 consecutive weeks; and should be willing to abandon other treatment interventions for the lower back during the intervention period. Potential participants were excluded if they: had undergone spinal surgery in the last 2 years; reported severe back pain that was hindering them in performing maximal isometric strength efforts; had radiation below the knee with signs of nerve root compression. ${ }^{7}$

\section{Intervention}

The high-intensity training group received an 8-week, progressive resistance exercise program for the isolated lumbar extensor muscle groups. The first two weeks provided two training sessions per week and the following six weeks one training session per week. The initial training load was set at approximately $50 \%$ of the maximal isometric lumbar extension strength of the participant, as measured at baseline. The goal of every training session was to perform 15 to 20 repetitions on the lower back machine. If the participant was able to perform more than 20 repetitions, a $2.5 \mathrm{~kg}$ weight was added in the next session. Conversely, the training load was lowered with $2.5 \mathrm{~kg}$ if the participant was unable to perform 15 repetitions. This training protocol is partly based on existing protocols, ${ }^{8-10}$ and partly on our own clinical experience.

The low-intensity training group received an 8-week, a nonprogressive, lowintensity resistance program. Throughout the eight weeks, the load was set at a maximum of $20 \%$ of the maximal isometric strength, as measured at baseline. Every session, one set of 15 or 20 repetitions was performed on the lower back machine. We assumed that resistance training on this load would not provide a physiological strength-training stimulus. 
In both the high-intensity strengthening program and the low-intensity strengthening program, training was carried out on a modified lower back machine (see Chapter 2, Figure 1). The main modifications consisted of a fixation of pelvis and hips to isolate the lower back, and a change in load curve during the training. Details about the modifications of the standard lower back machine have been published earlier. ${ }^{11}$

A load curve adjuster was used as an extra modification. The load curve adjuster allows adjustment of the workload throughout the full range of motion of the lower back. In our previous study, we saw that some participants of the highintensity training group were not able to make full extension, having inadequate strength in that particular part of the required range of motion of the lower back. ${ }^{11} \mathrm{~A}$ load curve adjuster with a beginning support or an end support made it possible for them to move in the whole range of motion until exhaustion.

All sessions in both programs were supervised by one and the same physiotherapist, who was kept unaware of the results of the outcome measurements. Every training session was preceded by a 5-minute warm-up on an arm/leg ergometer. During the training, special attention was paid to the technique in terms of pace and movement. The flexion and extension of the lower back had to be executed in the full individual range of motion. Movements had to be slow and controlled: moving in two seconds from maximal flexion to maximal extension (concentric contraction and lifting the weight), and returning from maximal extension to maximal flexion in four seconds. The weight load used and the number of repetitions during each session were recorded.

Participants who were assigned to the waiting list-group received no intervention for their low back pain during the first eight weeks.

\section{Outcome measures}

Primary outcomes in this study were global perceived effect and disability. Global perceived effect was expressed as the self-perceived percentage increase or decrease in the degree of back symptoms. Disability was measured by the original Roland-Morris Disability Questionnaire, a 24-item scale with scores ranging 
from 0 to 24 points, with high scores indicate higher disability as a result of low back pain. ${ }^{12}$ Secondary outcomes were: health-related quality of life, as measured by the Medical Outcome 36-item Short Form Health Survey (SF 36), 13,14 fear of movement/(re-)injury, as measured by the Tampa Scale for Kinesiophobia, ${ }^{15,16}$ and isometric back extension strength. The scores for the SF-36 range from 0 to $100 \%$ and indicate self-experienced health-related quality of life. Items are grouped into eight domains from which an overall summary score, a physical component score, and a mental component score can be derived.

A high score on each of the scales reflects a high level of self-experienced health. The Tampa Scale for Kinesiophobia is a 17-item scale with scores ranging from 17 to 68 points, measuring the extent to which a chronic back patient fears physical damage because of movement. High scores indicate a high degree of fear of movement/(re-)injury. Isometric back extension strength is evaluated with the same modified lower back training device as used in the exercise programs. A force transducer was used for the measurement of net isometric extension strength in five angles throughout the range of motion. Modifications and protocols used for the isometric strength measurements have been described in detail elsewhere. ${ }^{11}$

\section{Data analysis}

In order to calculate sample size we assumed a mean difference between the groups of 2 points ( $S D=4)$ improvement on the Roland-Morris Disability Questionnaire as clinically important. ${ }^{17}$ Assuming a SD of 4 , for power of 0.80 and a significance level of 0.05 , a target population of 64 participants was needed for each group, i.e., 192 participants in total.

Checks for missing and incorrect values were conducted prior to the analyses. All outcome measures were analysed by means of linear regression analysis (ie, analysis of covariance). Baseline values of the outcome measures were incorporated in the linear regression model as covariates, in order to correct for potential regression to the mean. 
First, we compared the outcomes at 8 weeks of the high-intensity traininggroup, the low-intensity training-group and the waiting list group. Second, participants of the waiting list group, who were randomly assigned to either a high-intensity strengthening program or a low-intensity strengthening program 8 weeks after initial randomization, were analysed together with the initial highintensity training group or the low-intensity training group. We compared the outcomes of these two newly-formed groups at 8 and 24 weeks follow-up. All analyses were carried out according to the intention-to-treat principle. Statistical significance was set at $p$ values of less than 0.05 .

\section{Results}

\section{Flow of participants through the trial}

Figure 1 shows the flow chart throughout the different phases of the trial. In the second half of 1998 and first half of 1999 a total of 71 volunteers responded to the advertisements and could be invited for the trial. Sixty-five of these 71 subjects met the inclusion criteria and were enrolled in the trial.

For practical reasons we were not able to lengthen the time for inclusion and had to close recruitment in July 1999. Thus data were collected from March 1999 through to February 2000. In our view, the main reason for failing to recruit the targeted sample size, despite our great source population, was the travel distance to the research and training department (on the average between 1 and 3 hours by car or train). The training location was in Utrecht, which is in the centre of the Netherlands. Most Royal Netherlands Army employees, however, work at locations in the countryside or even (temporary) abroad in peace-keeping missions. Table 1 presents characteristics of the participants. All participants were still working, moderately disabled and familiar with fitness and sport activities. There were only minor differences in demographic characteristics. According to these characteristics and the baseline values for the outcome measures (Table 2), we considered the three groups comparable at baseline. Six participants withdrew during the intervention period. One completed the first eight weeks of the waiting list period but could not be randomized as a result of a militairy mission; a further three withdrew during follow-up so that 56 participants completed the trial. 


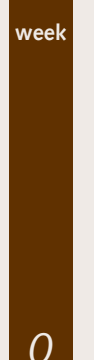

0

8
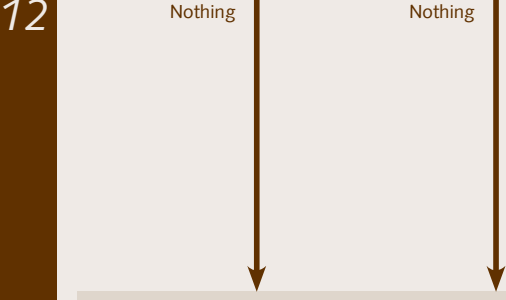

Measured disability (RDQ), Global Perceived Effect, Quality of Life (SF-360), Fear of Movement (TSK), Strength $(n=20)$ $(n=17)$

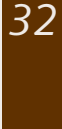
ment (TSK), Strength

$(n=20)$

Eligible participants $(n=71)$

6 Excluded (3 lack of time,

3 failed criteria

Measured disability (RDQ), Quality of Life (SF-36), Fear of Movement (TSK), Strength

Randomized $(n=65)$

$(n=21)$

$(n=21)$

Low-Intensity Training

Group

10 sessions in 8 weeks
Waiting-list Control

Group:

No training

Measured disability (RDQ), Global Perceived Effect, Quality of Life (SF-36), Fear of Move-

$(n=19)$

Randomized ( $n=20)$

$(n=21)$

High-Intensity Training

Group

6 sessions in 4 weeks

Low-Intensity Training

Group

6 sessions in 4 weeks

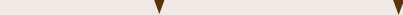

Measured disability (RDQ), Quality of Life SF-36), Fear of Movement (TSK), Strength

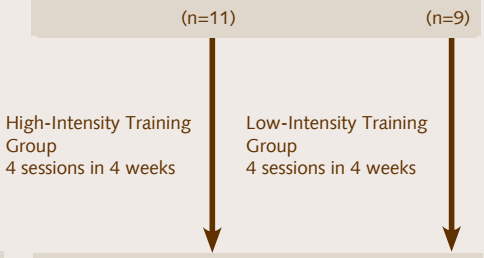

Measured disability (RDQ), Global Perceived Effect, Quality of Life (SF-360), Fear of Movement (TSK), Strength

( $n=11)$

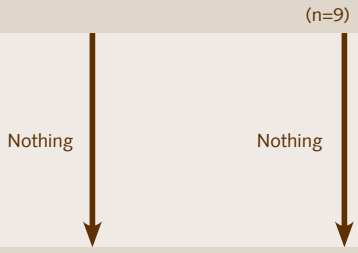

Measured disability (RDQ), Global Perceived Effect, Quality of Life (SF-360), Fear of Movement (TSK), Strength $(n=10)$

Figure 1. Flow chart of participants throughout the trial. 
Table 1. Demographic characteristics of study population presented in number of participants. Numbers between brackets represent percentages, unless otherwise stated.

\begin{tabular}{|c|c|c|c|}
\hline & HIT $(n=23)$ & LIT $(n=21)$ & WLC $(n=21$ \\
\hline \multicolumn{4}{|l|}{ Demographic Characteristics } \\
\hline Mean age in years (SD) & $44(10)$ & $42(10)$ & 41 (9) \\
\hline $\begin{array}{c}\text { Type of work: } \\
\text { sedentary } \\
\text { physical }\end{array}$ & $\begin{array}{r}19(83) \\
4(17)\end{array}$ & $\begin{array}{r}18(86) \\
3(14)\end{array}$ & $\begin{array}{r}19(90) \\
2(10)\end{array}$ \\
\hline \multicolumn{4}{|l|}{ Clinical Characteristics } \\
\hline $\begin{array}{l}\text { Time since first episode of LBP: } \\
\text { 3-12 months } \\
1-5 \text { years } \\
\geq 5 \text { years }\end{array}$ & $\begin{array}{r}2(9) \\
5(22) \\
16(70)\end{array}$ & $\begin{array}{r}2(10) \\
6(29) \\
13(62)\end{array}$ & $\begin{array}{r}2(10) \\
4(19) \\
15(71)\end{array}$ \\
\hline $\begin{array}{l}\text { Radiation } \\
\text { Earlier received therapies for LBP since } \\
\text { first episode: } \\
\text { no therapy } \\
\text { one therapy } \\
\text { more than one therapy }\end{array}$ & $\begin{array}{r}4(17) \\
6(26) \\
13(57)\end{array}$ & $\begin{array}{r}4(19) \\
7(33) \\
10(48)\end{array}$ & $\begin{array}{l}3(14) \\
7(33) \\
1152)\end{array}$ \\
\hline Current work absenteeism due to LBP & $7(30)$ & $5(24)$ & $8(38)$ \\
\hline $\begin{array}{l}\text { Frequency of work absenteeism due to } \\
\text { LBP in last year: }\end{array}$ & & & \\
\hline $\begin{array}{l}<1 \text { week in last year } \\
1-3 \text { weeks in last year } \\
\geq 3 \text { weeks in last year }\end{array}$ & $\begin{array}{c}19(83) \\
1(4) \\
3(13)\end{array}$ & $\begin{aligned} & 19(90) \\
& 1(5) \\
& 1(5)\end{aligned}$ & $\begin{array}{r}15(71) \\
3(14) \\
3(14)\end{array}$ \\
\hline
\end{tabular}

Abbreviations: HIT, High-Intensity Training group; LIT, Low-Intensity Training group; WLC, WaitingList control group. 


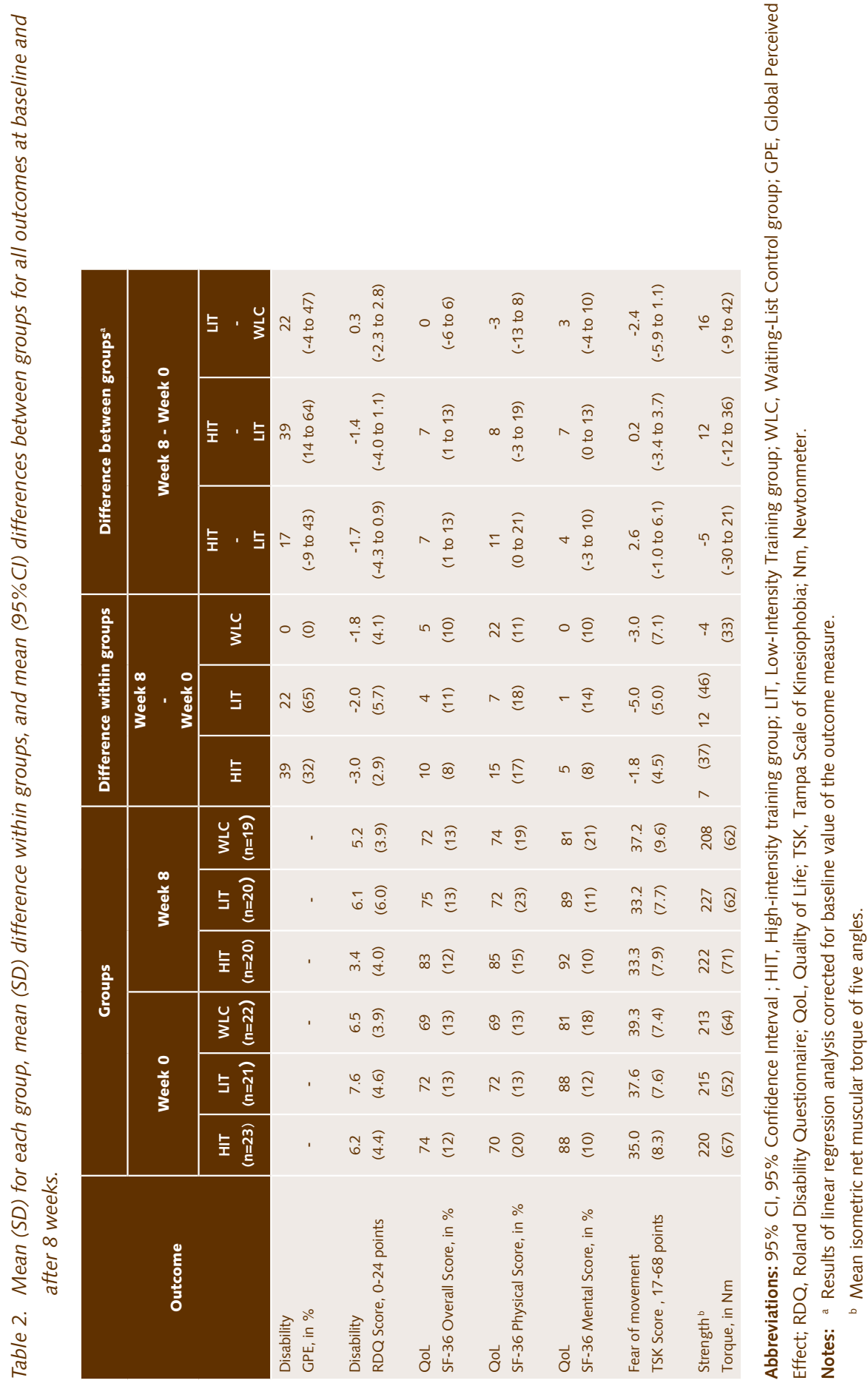




\section{Compliance with trial method}

A total of 31 participants received the high-intensity strengthening program (the initial high-intensity training group and half of the waiting list control group). Only one of this group missed one training session. The other 30 participants completed the whole program. A total of 28 participants took part in the lowintensity strengthening program (the initial low-intensity training group and half of the waiting list control group). Four of this group missed one session and only one missed three training sessions. Twenty-three participants completed the program. No co-interventions were reported during both programs and the waiting list period.

Two participants who had attended the high-intensity strengthening program (the initial high-intensity training group and half of the waiting list control group) and three participants who had attended the low intensity strengthening program (the initial low-intensity training group and half of the waiting list control group) sought professional medical help for their low back pain during follow-up. Furthermore, 11 participants who had attended the high-intensity strengthening program and five participants of the low-intensity strengthening program continued with exercise training, and some of them continued with the specific training. Seven participants of the high-intensity strengthening program and nine participants of the low-intensity strengthening program did not receive any intervention during follow-up.

\section{Effect of intervention}

Group data for the high-intensity training, low-intensity training and waiting list control groups at two measurement times (Week 0 and 8 ) as well as within- and between- group data are presented in Table 2. A mean difference in improvement of $7 \%(95 \% \mathrm{Cl} 1$ to 13$)$ in SF-36 overall score was found in favour of the high-intensity training group when compared to the low-intensity training group. Self-assessed decrease of back symptoms was on average 39\% (95\% Cl 14 to 64 ) greater in the high-intensity training group when compared to the waiting list control group. A mean difference in overall SF-36 score of 7\% ( $95 \% \mathrm{Cl} 1$ to 13 ) was found in favour of the high-intensity training group when compared to the waiting list control group. No statistically significant differences 
in improvement between the three groups were found for the other outcome at 8 weeks. In an additional analysis, we compared the high-intensity training and low-intensity training groups with the addition of the participants of the waiting list control group, who were randomly assigned to high-intensity strengthening or low-intensity strengthening 8 weeks after the initial randomization. These newly-formed groups were not different with regard to demographic characteristics at baseline (data not shown). Group data for the high-intensity training and low-intensity training groups at 3 measurement times (Week 0, 8, and 24) as well as within- and between-group data are presented in Table 3. The differences in improvements between the high-intensity training and low-intensity training group at 8 and 24 weeks were not statistically significant.

\section{Discussion}

In this study we compared the effects of a high-intensity and a low-intensity strengthening program for the lumbar extensor muscles with a waiting list control group in a population of army personnel suffering from nonspecific low back pain. Most of the comparisons between the exercise groups and the waiting list control group for global perceived effect, disability, health-related quality of life, fear of movement/(re-)injury and isometric back strength showed point estimates which did not favour the two exercise groups. Immediately after intervention at 8 weeks, the high-intensity strengthening program produced a greater perceived effect than the low-intensity strengthening program. In addition, the high-intensity program produced a greater quality of life than the low-intensity strengthening program (in both analyses) but this difference had disappeared 16 weeks later.

Overall, the generally claimed beneficial influence of exercise for chronic low back pain, as suggested in systematic reviews and practitioner guidelines, 1,18,19 is only partly supported by the results of this trial. Significant differences in improvement were limited to global perceived effect and health-related quality of life in the short-term ( 8 weeks) when comparing the groups with the highest contrast (high-intensity training group and waiting list control group).

Despite the robustness of the study design (randomization procedure, waiting 


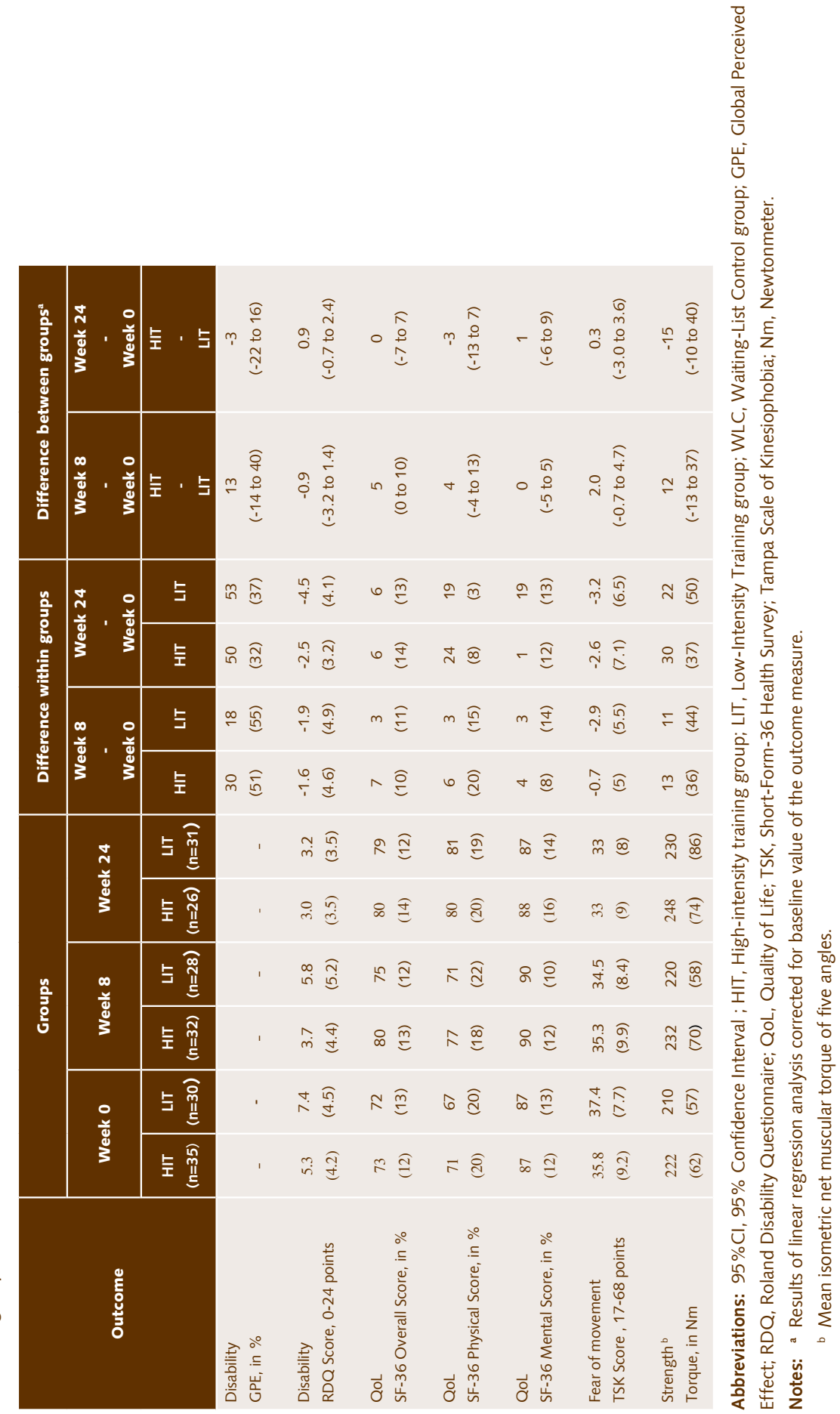


list control group), our trial has some limitations. The main limitations of this study were the lack of statistical power and the relatively short follow-up period. The open voluntary recruitment of participants might further confine the generalisability of findings by limiting a direct comparison with a healthcare-seeking population. Voluntary recruitment might also be the cause of an overrepresentation of participants with only a moderate disability level. This is illustrated by the mean Roland-Morris Disability Questionnaire score at baseline for the total study group, which ranged from 6.2 to 7.6 points (out of a maximum of 24 points). The moderate disability level may have elicited floor effects. As already mentioned, the exercise programs may be more attractive for people with active lifestyle habits, which also means that they are less attractive and applicable to other populations.

The intensity of both exercise programs was based on existing protocols of the Royal Netherlands Army and on our own clinical experience. Given the lack of effects for strength gain, one might question whether the magnitude of the strength stimulus in the high-intensity training group, i.e., one set of 15-20 repetitions twice weekly for two weeks and once after this for another 6 weeks, was high enough to cause physiological training effects. On the other hand, the participants of this trial were not healthy adults and we expected them to be, to some extent, untrained because of their back pain, which legitimises a more careful approach. Another intensity issue concerns the total duration of all training sessions. A systematic review on strategies for using exercise therapy for chronic low back pain showed that high-dose ( $>20$ hours) exercise programs are more effective than low-dose ( $<20$ hours) exercise programs. ${ }^{20}$ The programs used in the present trial are low-dose programs according to the definitions of this systematic review which was published after our trial was designed.

The application of exercise therapy for low back pain in clinical practice varies widely with regard to the type of exercises used, the intensity, the frequency, the duration, and whether the exercises are combined with other treatment modalities. ${ }^{18,20}$ Seven randomized controlled trials specifically investigated the effects of lumbar back muscle training for low back pain as we did in this study. ${ }^{22-}$ ${ }^{28}$ Chok et $\mathrm{al}^{21}$ evaluated the effects of endurance training of the trunk extensor 
muscles 3 times a week for 6 weeks, compared to no exercises, and found no significant differences between the two groups at 6 weeks. Hansen et al22 compared 8 sessions of intensive dynamic back muscle exercises with conventional physiotherapy and placebo in a randomized controlled trial. They found that the intensive back exercises and physical therapy groups were significantly more effective over a 12-month period than the placebo-controlled group that had received traction and hot packs. In a trial of 105 subjects, ${ }^{23,24}$ thirty sessions of intensive dynamic back exercises over 3 months were compared with a similar program with $1 / 5$ th of the intensity and a program of mild exercises and passive modalities. The results for pain, disability and physical impairment (i.e., back endurance and mobility) were in favor of the intensive exercise group. Kankaanpaa et $\mathrm{a}^{25}$ found that low back pain patients who underwent dynamic extensor training had significantly greater in pain and disability than a group that underwent passive modalities. Other studies indicate that back muscle exercises are no more effective than other types of exercise treatments. ${ }^{6,26,27}$ The picture becomes even more complicated when differences in treatment intensity, treatment frequency, type of exercises, study population, follow-up period, and outcome measures between these studies are taken into account.

What emerges from these summarised study results is the tendency that strengthening of the back extensor muscles is more effective than passive modalities or doing nothing. This hypothesis is partially confirmed by the results of the present study. Further exploration is needed with regard to the optimal type, intensity, and frequency of isolated back extensor exercises, as well as to the mediating role of pain-related fear in exercise programs. ${ }^{28}$ There is still room for methodologically sound randomized controlled trials with sufficient statistical power studying the effects of isolated back extensor exercises for low back pain.

Acknowledgements: The authors would like to thank Peter de Putter for the software of the measurement of net isometric extension strength. Also, we would like to thank kol R. Roelofs, for authorizing this study. 


\section{References}

1. Koes BW, van Tulder MW, Ostelo R, et al. Clinical guidelines for the management of low back pain in primary care: an international comparison. Spine 2001;26:2504-13.

2. Rissanen $\mathrm{A}$, Kalimo $\mathrm{H}$, Alaranta $\mathrm{H}$. Effect of intensive training on the isokinetic strength and structure of the lumbar muscles in patients with chronic low back pain. Spine 1995;20:333-40.

3. Storheim K, Holm I, Gunderson R, et al. The effect of comprehensive group training on cross-sectional area, density, and strength of paraspinal muscles in patients sicklisted for subacute low back pain. J Spinal Disord Tech 2003;16:271-9.

4. Verna JL, Mayer JM, Mooney V, et al. Back extension endurance and strength: the effect of variable-angle roman chair exercise training. Spine 2002;27:1772-7.

5. Mannion AF, Muntener $M$, Taimela $S$, et al. A randomized clinical trial of three active therapies for chronic low back pain. Spine 1999;24:2435-48.

6. Mannion AF, Junge A, Taimela $S$, et al. Active therapy for chronic low back pain: part 3. Factors influencing self-rated disability and its change following therapy. Spine 2001;26:920-9.

7. Faas AC, Koes BW, van den Hoogen JM, et al. [NHG-Standaard Lage-Rugpijn]. Huisarts Wet 1996;39:18-31

8. Pollock ML, Leggett SH, Graves JE, et al. Effect of resistance training on lumbar extensor strength. Am J Sports Med 1989;17:624-9.

9. Graves JE, Pollock ML, Foster D, et al. Effect of training frequqncy and specificity on isometric lumbar extension strength. Spine 1990;15:504-9.

10. Graves JE, Webb DC, Pollock ML, et al. Pelvic stabilization during resistance training: Its effect on the development of lumbar extension strength. Arch Phys Med Rehabil 1994;75:210-5.

11. Helmhout $\mathrm{PH}$, Harts CC, Staal JB, et al. Comparison of a high-intensity and a lowintensity lumbar extensor training program as minimal intervention treatment in low back pain: a randomized trial. Eur Spine J 2004;13:537-47.

12. Roland M, Morris R. A study of the natural history of back pain. Part I: development of a reliable and sensitive measure of disability in low back pain. Spine 1983;8:141-4.

13. Shmueli A. The SF-36 profile and health-related quality of life: an interpretative analysis. Qual Life Res 1998;7:187-95.

14. Van der Zee K, Sanderman R. [Het meten van de algemene gezondheidstoestand met de RAND-36: Een handleiding.] Noordelijk Centrum voor Gezondheidsvraagstukken, Groningen, 1993.

15. Kori SH, Miller RP, Todd DD. Kinisiophobia: A new view of chronic pain behavior. Pain Manag 1990;Jan/Feb:35-43.

16. Vlaeyen JW, Kole-Snijders AM, Boeren RG, et al. Fear of movement/(re)injury inchronic low back pain and its relation to behavioral performance. Pain 1995;62:363-72.

17. Stratford PW, Binkley JM, Riddle DL, et al. Sensitivity to change of the Roland-Morris Back Pain Questionnaire: part 1. Phys Ther 1998;78;1186-96.

18. Hayden JA, van Tulder MW, Malmivaara A. Exercise therapy for treatment of nonspecific low back pain. Cochrane Database Syst Rev: CD000335, 2005.

19. Staal JB, Hlobil H, van Tulder MW, et al. Occupational health guidelines for the management of low back pain: an international comparison. Occup Environ Med 
2005;60: 618-26.

20. Hayden JA, van Tulder MW, Tomlison G. Systematic review: strategies for using exercise therapy to improve outcomes in chronic low back pain. Ann Intern Med 2005;142:776-85.

21. Chok B, Lee R, Latimer J, et al. Endurance training of the trunk extensor muscles in people with subacute low back pain. Physical Therapy 1999;79:1032-42.

22. Hansen FR, Bendix T, Skov P, et al. Intensive, dynamic back-muscle exercises, conventional physiotherapy, or placebo-control treatment of low-back pain. A randomized, observer-blind trial. Spine 1993;18:98-108.

23. Manniche $C$, Hesselsoe $G$, Bentzen $L$, et al. Clinical trial of intensive muscle training for chronic low back pain. Lancet 1988;2:1473-6.

24. Manniche C, Lundberg E, Christensen I, et al. Intensive dynamic back exercises for chronic low back pain: a clinical trial. Pain 1992;47:53-63.

25. Kankaanpaa M, Taimela S, Airaksinen $O$, et al. The efficacy of active rehabilitation in chronic low back pain. Effect on pain intensity, self-experienced disability, and lumbar fatigability. Spine 1999;24:1034-42.

26. Mannion AF, Muntener $M$, Taimela $S$, et al. Comparison of three active therapies for chronic low back pain: results of a randomized clinical trial with one year follow-up. Rheumatol 2001;40:772-8.

27. Rittweger J, Just K, Kautzsch K, et al. Treatment of chronic lozwer back pain with lum bar extension and whole-body vibration exercise: a randomized controlled trial. Spine 2002;27:1829-34.

28. Smeets RJ, Vlaeyen JW, Kester AD, et al. Reduction of pain catastrophizing mediates the outcome of both physical and cognitive-behavioral treatment in chronic low back pain. Journal of Pain 2006;7:261-71. 


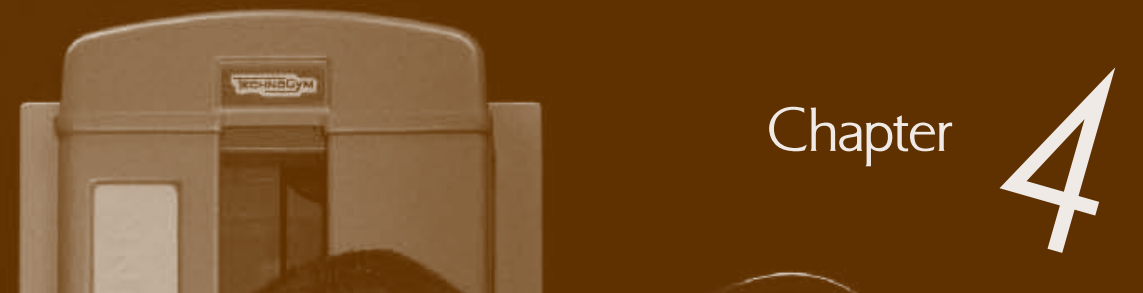

\title{
Rationale and design of a multicenter randomized
}

\author{
controlled trial on a "minimal intervention
}

"in Dutch army personnel with nonspecific

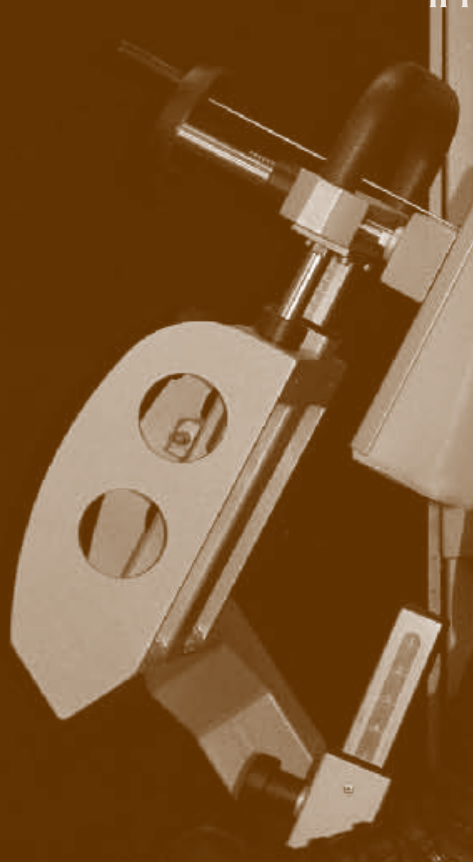

This Chapter has been published as:

Rationale and design of a multicenter randomized controlled trial on a "minimal intervention" in Dutch army personnel with nonspecific low back pain [ISRCTN19334317]

P.H. Helmhout ${ }^{1}$, C.C. Harts' ${ }^{1}$ J.B. Staal ${ }^{2}$, R.A. de Bie ${ }^{2}$,

Department of Training Medicine and Training Physiology, Royal Netherlands Army, Utrecht, The Netherlands

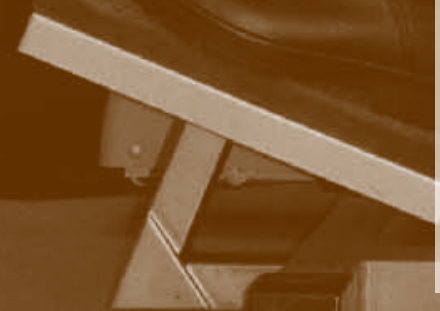

2 Department of Epidemiology and Caphri Research School,

Maastricht University, Maastricht, The Netherlands 


\section{Abstract}

Background: Researchers from the Royal Netherlands Army are studying the potential of isolated lumbar extensor training in low back pain in their working population. Currently, a randomized controlled trial is carried out in five military health centers in The Netherlands and Germany, in which a 10-week program of not more than 2 training sessions (10-15 minutes) per week is studied in soldiers with nonspecific low back pain for more than 4 weeks. The purpose of the study is to investigate the efficacy of this "minimal intervention program", compared to usual care. Moreover, attempts are made to identify subgroups of different responders to the intervention.

Methods: Besides a baseline measurement, follow-up data are gathered at two short-term intervals ( 5 and 10 weeks after randomization) and two long-term intervals ( 6 months and one year after the end of the intervention), respectively. At every test moment, participants fill out a compound questionnaire on a stand-alone PC, and they undergo an isometric back strength measurement on a lower back machine. Primary outcome measures in this study are: selfassessed degree of complaints and degree of handicap in daily activities as a result of back pain. In addition, our secondary measurements focus on: fear of movement/(re-) injury, mental and social health perception, individual back extension strength, and satisfaction of the patient with the treatment perceived. Finally, we assess a number of potential prognostic factors: demographic and job characteristics, overall health, the degree of physical activity, and the attitudes and beliefs of the physiotherapist towards chronic low back pain.

Discussion: Although a substantial number of trials have been conducted that included lumbar extension training in low back pain patients, hardly any study has emphasized a minimal intervention approach comparable to ours. For reasons of time efficiency and patient preferences, this minimal sports medicine approach of low back pain management is interesting for the population under study, and possibly for comparable working populations with physical demanding job activities. 
List of abbreviations: $R N L A=$ Royal Netherlands Army, $R C T=$ randomized controlled trial, $L B P=$ low back pain, $B S=$ Back Strength intervention, UC = Usual Care intervention, rep $=$ repetition on back extension machine

\section{Background}

\section{Back pain treatment in the military setting: a "minimal intervention approach"}

For the last ten years, clinical researchers from the Royal Netherlands Army (RNLA) have studied the potential of physical training modalities in preventing and alleviating nonspecific low back pain (LBP) in their working population. Many military and civilian job functions in the RNLA involve heavy manual material handling and therefore spine-loading activities. In general, the incidence of back problems is higher in physically demanding tasks than in sedentary activities ${ }^{1}$. Concordantly, the incidence of LBP in the RNLA is high. Acute LBP is the primary reason for soldiers to visit the general practitioner at a military health center. Chronic nonspecific LBP, defined as having complaints for at least 12 weeks, is one of the three most diagnosed disorders during consulting hours of Dutch military company doctors, and takes on average $15 \%$ of their weekly consulting hours time.

Currently, there is strong evidence that exercise therapy is more effective than usual care ${ }^{2}$. Exercise therapy is a major part of the standard treatment by physiotherapists in the RNLA, involving an active role of the patient. In the experience of our health professionals (practitioners and physiotherapists), this active approach fits in well with the attitudes and beliefs of the target population: soldiers are taught to be aware of their physical abilities and limitations from the moment they enlist for the army. After all, recruits who do not successfully complete their basic training cannot progress on to a career as a soldier.

The specific character of military tasks nowadays (e.g., preparation for crisis management operations abroad) interferes with the schedules of rehabilitation programs for back-injured soldiers which, in general, take several days per week over a considerate number of consecutive weeks. Therefore, the search 
for effective and (time-) efficient exercise therapy protocols has led us to a specific form of lumbar extension training. Each training session consists of no more than 5 to 10 minutes training of the isolated lumbar extensors on a special training device.

\section{Arguments for this study}

For a number of years, we have experience with high-intensive, isolated training of the lumbar extensors in military personnel with nonspecific LBP, by using a special training device. In this a sports medicine approach is followed, partly according to established exercise protocols $s^{3,4}$, in which three key principles are emphasized: (1) isolation of the lumbar extensors through fixation of the pelvis and thighs; (2) training in the individual's full range of motion; (3) avoiding "sticking points" in the training load - i.e., points in the range of motion in which a relatively high resistance is experienced - by tuning the load curve of the weight stack to the individual's strength curve.

In individual cases, we observed satisfying to sometimes excellent results in terms of pain relief and functional restoration, when giving a training stimulus of no more than 5 to 10 minutes ( 1 to 2 training sessions) per week. These findings, however, need to be confirmed in a randomized controlled trial.

Four main reasons led to the choice of doing research on the efficacy of this sports medicine approach for our working population with LBP. First, recent systematic reviews indicate that exercise therapy is a successful approach for the restoration of chronic and recurrent LBP, at least in the short-term ${ }^{2,3}$. However, higher quality studies generally show a lack of treatment specificity of various exercise modalities, such as aerobic exercises, strength and endurance reconditioning or mobilizing exercises ${ }^{4,5}$. Moreover, controversy remains regarding the impact of a training stimulus, in terms of intensity, duration and frequency, on the reduction of LBP. Different explanations for this lack of specificity are given in the literature, such as nonspecific, more centrally induced training effects (e.g., a shift in pain perception ${ }^{5}$ ), or large heterogeneity in the chosen study populations ${ }^{6}$. If, indeed, no specific dimension or type of exercise therapy is superior to one another in producing optimal therapeutic outcomes, 
other aspects are more relevant when introducing an intervention program, such as: treatment affinity, expectation and compliance of patient and provider, costs, facilities, and personnel capacity. From this perspective, back strength and endurance training in CLBP patients with the use of training devices is an interesting concept for our military population.

RNLA personnel are, from their very first initial military education, used to participate in physical exercise programs, including progressive resistance training on exercise machines. The RNLA is well equipped with an extensive line of modern fitness devices on all major military locations throughout the country, including state-of-the-art lower back machines. Moreover, protocolized treatment sessions with our training device take no longer than 5 to 10 minutes once or twice a week from both patient and provider, compared to (on an average) 30 minutes in regular treatment sessions. We expect this time efficiency to be highly appreciated by our personnel, who work in a typical military culture of "running into extremes": relatively quiet (maintenance) periods on the military base are interspersed with extremely busy periods shortly before and during outof-area operations. For several target groups, longstanding and time-consuming rehabilitation programs are out of the question. For instance, recruits who drop out of their initial training because of (back) injuries, need to return as quick as possible to prevent a stagnation in their military career. For soldiers standing by for military operations, mission-related activities are prioritized when being commanded to be prepared within the next weeks.

A second reason for our approach is that the majority of studies on LBP management consist of multimodal interventions, which include physical, behavioral, educational and/or ergonomic elements. To obtain a better view on the (relative) efficacy of either of these concepts, unimodal intervention programs like ours need to be evaluated ${ }^{7,8}$. Besides, we strongly believe that exercise as the primary entrance for restoring back function has a wide span of treatment effects, including improvements for cognitive and/pr behavioral variables. Although exercise has a primary goal of improving functioning of targeted tissues, successful completion of exercise protocols in the presence of chronic pain may for example lead to a reduction in pain-related fears. As exercise on a training 
device is mostly based on measured performance (number of kilograms and repetitions), patients are continuously given numerical feedback regarding their increasing physical capacities ${ }^{9}$. An increased awareness of improving physical capacities may draw their attention away from pain and suffering.

Third, the choice for a particular intervention approach depends in many cases on the stage and severity of the back problem, the extent to which psychosocial aspects are involved, and the needs and preferences of the patient. For instance, behavioral therapy is mainly focused on issues that are prevalent in chronic patients, such as low feelings of self-control or fear of movement/(re-) injury. Since the population of the present study, military employees of the RNLA, is a working population with mostly short-term, intermittent and moderately severe LBP, we chose to apply a more physical approach. As we have seen in our previous research (see the next paragraph), this links well with the health perceptions of our target population, in which perceived health problems were not severe and much more focused on physical than on mental aspects.

Fourth, the efficacy of isolated extension training in chronic back patients has been studied by several other research groups as well ${ }^{10}$. Although promising results were reported regarding lumbar strength improvements and pain relief, several methodological shortcomings hinder solid interpretation of these findings. Most problems encountered were a small sample size, lack of randomization, lack of long-term follow-up results, variation in study populations (e.g., healthy volunteers, employees receiving worker's compensation), and inadequate or missing control groups ${ }^{7,10-14}$. In a review on lumbar extension training with MedX-equipment in LBP patients, Miltner et $\mathrm{al}^{8}$ conclude that more controlled studies are needed "to delineate further the role of isolated lumbar extension exercise for the treatment of LBP and to test the efficacy compared to other methods of care."

\section{Earlier research on our minimal intervention approach}

Especially in recent years, we have scientifically studied the potential of our sports medicine approach. In two previous trials we compared the efficacy of a high-intensive, progressive resistance training program of the isolated lumbar 
extensors, with a low-intensive, nonprogressive program of the same extend, in a group of workers with nonspecific LBP. Total intervention time of both "minimal intervention programs" was limited to 14 sessions of 5 to 10 minutes, over a period of 12 weeks ( $1^{\text {st }}$ trial) or 8 weeks ( $2^{\text {nd }}$ trial).

In the first trial, we were unable to demonstrate that either of the two training programs was superior in alleviating back complaints ${ }^{15}$. However, the magnitude of the improvements in back function found in this study were in line with those reported in other studies, which used more extended (multimodal) exercise programs. Therefore, it would be interesting to compare the efficacy of our minimal intervention program with the usual care RNLA personnel with nonspecific LBP.

Moreover, the results of our first trial indicated that some individuals with LBP might benefit more from an aggressive approach, showing a trend towards a higher improvement rate (self-assessed percentage decrease in complaints) directly after the "minimal intervention" treatment, as well as a higher compliance to the treatment and a higher willingness to participate in physical exercise on the longer term. In the present multicenter study, we aim at identifying relevant subgroups of patients that show higher success rate as a result of this training approach.

\section{Methods}

\section{Study Design and Population}

In a randomized, single-blinded multicenter trial, we evaluate the efficacy of progressive, isolated resistance training of the lumbar extensor muscles, compared to the usual care. The trial started in April 2002; data will be collected till the end of 2005. The source population $(n=23,000)$ consists of military employees of the RNLA. Our in- and exclusion criteria are listed in Table 1. Recruitment of participants takes place during regular office hours of the military general practitioner. A brief outline of the study design is presented in Figure 1. 


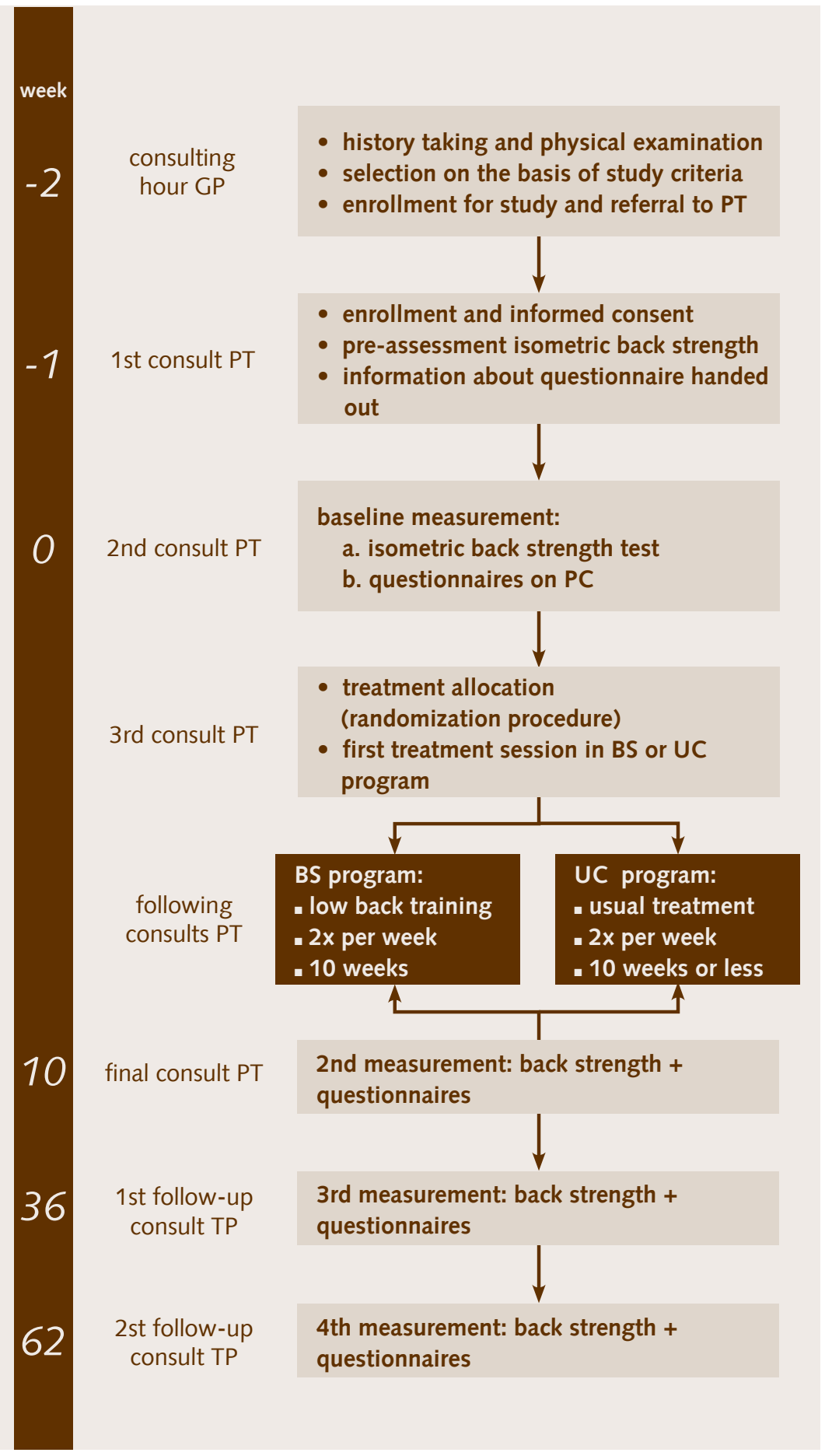

Figure 1. Flow chart of the different phases of the multicenter trial. 
Table 1. In- and exclusion criteria of the study.

\section{Inclusion criteria}

- military employees of the RNLA between the age of 18 and 54 years

- at least 4 weeks of continuous or recurrent (at least 3 times a week) episodes of LBP

- pain localized between posterior iliac crests and angulus inferior scapulae

- availability to visit the local military health center 2 times a week during 10 consecutive weeks, with no more than two sessions of absence due to job-related activities (e.g. military exercise, course, leave)

- willingness to abandon other treatment interventions for the lower back during the intervention period

- signed informed consent

\section{Exclusion criteria}

- spinal surgery in the last 2 years

- specific treatment for LBP in the last 4 weeks (e.g., physiotherapy, manual therapy)

- severe LBP which hinders in performing maximal isometric strength efforts

- specific LBP, defined as herniated disc, ankylosing spondylitis, spondylolisthesis or other relevant neurological diseases

\section{Study sites}

Almost every military location in the Netherlands has a health center, in which general practitioners, dentists and physiotherapists give primary care to military personnel of the RNLA. For the present study we selected five military health centers on the basis of: (1) representing a major part of the total military population; (2) holding at least two full-time physiotherapists; (3) the willingness to unconditionally participate in the project.

The selected military health centers include the following locations:

- Amersfoort, in the middle-east of the Netherlands: approximately $15 \%$ younger soldiers on stand-by for military operations abroad (18-25 years), $25 \%$ military instructors (35+ years), 10\% staff personnel (40+ years), and $46 \%$ civilian workers from supporting units ( $40+$ years); 
- Den Haag, in the west of the Netherlands: approximately $90 \%$ older staff personnel (40+ years) at office work;

- Oirschot, in the south of the Netherlands: approximately $75 \%$ younger soldiers in their initial military education or on stand-by for military operations abroad (18-25 years), 25\% military instructors and staff personnel (35+ years);

- Schaarsbergen, in the south-east of the Netherlands: approximately $65 \%$ younger soldiers in their initial military education or on stand-by for military operations abroad (18-25 years), and 30\% staff personnel (35+ years);

- Seedorf, in the north-west of Germany (part of the 1 German/Netherlands Corps): approximately $65 \%$ younger soldiers on stand-by for military operations abroad (18-25 years), 20\% staff personnel (35+ years), and 10\% civi lian workers from supporting units (40+ years).

\section{Study population}

All general practitioners at each of the selected study centers identify potential subjects from among their clinic's patients, according to the aforementioned criteria. Each subject is submitted to regular history taking and physical examination by the physician; checklists with standard elements for LBP have been provided to all physicians. If eligible, patients are informed about the study. All relevant information from the intake is written down in a referral; a visit to one of the physiotherapists of the center is planned within the next days.

At the $1^{\text {st }}$ visit to the physiotherapist, patients receive further information about the trial. A pre-assessment of the isometric back strength is taken. A written explanation of one of the questionnaires, in which patients have to choose the three most disabled daily activities because of back pain, is given them to take home, which allows them to make a well-considered choice at the next visit. Written informed consent is obtained from all patients who are willing and eligible to participate. At the $2^{\text {nd }}$ visit, participants undergo a baseline measurement consisting of a compound questionnaire and an isometric back strength test.

At the third visit, patients are randomized into either the back strengthening group or usual care group. Directly after randomization, the first treatment sessi- 
on starts. Patients are allowed to withdraw from the study at any time, although the importance of full compliance of every participant is emphasized.

Randomization is done by means of a computer-generated table of random numbers per study center, using a block size of ten. Prestratification is applied for the duration of the back complaints, with a cut-off point of 12 weeks, suspecting duration of complaints to influence the individual response to the exercise program. One intervention group receives a back-strengthening program; the other group receives a usual care program.

The randomization is concealed, which means that the treating physiotherapist obtains the allocated treatment by means of a computer software program, by entering the name and military registration number of the patient. The study protocol was approved by the Medical Ethics Committee of the Netherlands Central Military Hospital.

\section{Study interventions}

\section{Back Strengthening Program}

Subjects allocated to the back-strengthening program (BS) undergo a 10-week, progressive resistance-training program of the isolated lumbar extensor muscle groups. The program includes 14 training sessions (2 days per week) and 3 isometric back strength tests (in week 1,5, and 10). The initial training load is set at approximately $35 \%$ of the maximal isometric back extension strength of the participant, measured at baseline. The goal of every training session is to perform 15 to 20 repetitions (reps) on the lower back machine, equivalent to approximately $50 \%$ and $70 \%$ of the one-repetition maximum (1 RM) respectively. If the subject is able to perform a higher number of reps, $2,5 \mathrm{~kg}$ weight is added the next time. Vice versa, if the participant is unable to perform the minimal number of reps, the subsequent training load is lowered with $2,5 \mathrm{~kg}$. This training protocol is based on existing protocols $5^{5,18}$, as well as on our own experiences. A comprehensive training protocol can be obtained from the authors.

Training sessions are carried out on a Total Trunk Rehab (Technogym Inc, Italy). This lower back machine is equipped with a knee-lock system and a thigh- 
restraining belt to immobilize both hips and thighs, allowing the participant only to move the isolated lower back.

All training sessions are conducted as much as possible by the same physiotherapist. The physiotherapist pays special attention to the execution of the training in terms of pace and movement. The flexion and extension of the lower back has to be executed in the full range of motion of the participant, and movements have to be slow and controlled (moving in two seconds from maximal flexion to maximal extension when lifting the weight stack, and returning from maximal extension to maximal flexion in four seconds when lowering the weight stack). During this movement, emphasis is put on the hollowing and flattening of the lumbar lordosis. Every training session is preceded by a 5-minute allbody warming-up on an arm/leg ergometer (Schwinn Airdyne Pro, Balans Inc., Nieuwegein, The Netherlands). The weight load used and the number of reps completed during each training session are recorded.

\section{Usual Care Program}

Subjects allocated to the usual care program (UC) receive regular physical therapy for their lower back for at most 10 weeks, or earlier when the patient indicates to be free of complaints. Based on the physiotherapist's judgment, this could include "hands-on" treatment (e.g., passive mobilizing and pain-cushioning techniques, manual therapy) and/or "hands-off" treatment (e.g., exercise therapy, individual education and instruction on the back function). In the RNLA, active therapy forms are favored. To increase the contrast between both intervention programs, physiotherapists are not allowed to use the lower back machine in their usual care. Patients are not allowed to undergo co-treatment beside the interventions programs during the treatment period, nor exercise on equipment that mimics the specific components of the lower back machine. Therapeutic activities in every therapy session as well as the number of sessions are written down on a form.

\section{Outcome measurements}

In this study we have chosen primary outcome measures that are most relevant to the patient and clinician: self-assessed degree of complaints and degree of 
handicap in daily activities because of LBP. In addition, our secondary measurements focus on several other LBP-related areas, like kinesiophobia or mental health perception.

We have included some potential prognostic factors into our measurements, i.e., characteristics of the patient that possibly influence the effect of the intervention: job characteristics, overall health, physical activity, patient satisfaction with the allocated treatment, and attitudes and beliefs of the physiotherapist. This trial is mainly focused on the efficacy of our minimal intervention strategy, and not on unraveling the physiological or psychological working mechanisms of isolated lumbar extension training on LBP. Nevertheless, by including potential prognostic variables, starting points for further research into the "black box" of this type of intervention could be identified.

\section{Baseline characteristics}

The following demographic variables are registered during the intake: age, time since first episode of LBP, pain radiation, treatment history, and work absenteeism because of LBP in the last year. Moreover, the status of the patient before treatment, in terms of job aspects, overall health and the degree of physical activity, is assessed using a compound questionnaire.

Several job characteristics, i.e., content, relation with superior and colleagues, conflicts, and physical aspects of the job, are measured using subscales of a validated Dutch version of the Job Content Questionnaire ${ }^{16}$. Physical aspects of former jobs are assessed using one item of the Dutch Musculoskeletal Questionnaire ${ }^{17}$.

Overall health is assessed with one item from the MOS 36-item Short Form Health Survey ${ }^{18}$ : "What do you think, in general, of your health?" (1=bad, 2=moderate, $3=$ good, 4=very good, 5=excellent).

The degree of physical activity is measured with the Short Questionnaire to Assess Health Enhancing Physical Activity ${ }^{19}$, a validated and fairly reliable 5-item questionnaire. 
To assess the attitudes and beliefs of the participating physiotherapists about the relationship between low back pain and function before the start of the study, we use the Pain Attitudes and Beliefs Scale for Physiotherapists ${ }^{20}$. These attitudes and beliefs are believed to influence the physiotherapist's commitment to a certain treatment approach.

\section{Primary outcome measures}

Global perceived effect ${ }^{21}$ is measured by self-assessment on a 7-point scale (1 = completely recovered, 2 = much improved, 3 = slightly improved, $4=$ no change, $5=$ slightly worsened, $6=$ much worsened, $7=$ vastly worsened). We defined scores of 1 and 2 as a clinically important change.

Patient-specific functional status ${ }^{22}$ is measured by a questionnaire following a patient-specific approach. At baseline, individual patients select three main complaints, i.e., frequent activities which they perceive as important in their daily life, but which are hampered by their back pain. Patients rate the severity of these three complaints on a $100 \mathrm{~mm}$ visual analogue scale at each test moment. The responsiveness of the questionnaire is fairly good, with an area under the ROC-curve of 0.82 , and with mediate correlations with the Roland Disability Questionnaire ( $r=0.69-0.75)$ and the Visual Analogue Scale $(r=0.70-0.80)^{21}$. Low back specific functional status is measured by the validated Dutch version of the Roland Disability Questionnaire ${ }^{23,24}$, a widely used 24-item scale that reflects the functional disability because of LBP. Test-retest reliability of this scale is considered good for three weeks $(r=0.83)$ and six months $(r=0.72)$, respectively ${ }^{25}$.

\section{Secondary outcome measures}

Fear of movement or re-injury is measured by the Tampa Scale for Kinesiopho$\mathrm{bia}^{26}$, a 17-item scale to obtain a score for the extent to which a chronic back patient fears (new) physical damage because of physical activity. The Dutch version of this questionnaire has been found sufficient reliable and valid ${ }^{27,28}$.

Mental health is measured by the Dutch translation of the 12-item General Health Questionnaire ${ }^{29,30}$, assessing problems concerning psychological distress, like depression, sleep deprivation, stress coping, and self confidence. 
Test-retest reliability of the scale is high in a general population, with reported Cronbach's alpha coefficients between 0.86 and $0.90^{30}$.

Social health is measured by a subscale of the Impact on Participation and Autonomy Questionnaire ${ }^{31}$, focusing on the influence of the disability on social relationships; Cronbach's alpha for this factor is 0.87 .

Overall work status is measured by a 4-level item ( $1=$ "currently working full duties and/or able to do all of my regular home duties", $2=$ "able to do all work and/or home duties but it causes extra pain", 3="on restricted duties at work and/or need help with some of my home duties", 4="off work and/or need help with most of my home duties").

Individual back extension strength progression was evaluated using repeated isometric measurements on the lower back training and testing machine. A detailed description of these measurements can be find elsewhere ${ }^{15}$.

Patient satisfaction is measured at the end of the treatment program by two 3-level items and one 5-level item, in which the degree of satisfaction with the allocated treatment is assessed: (a) "Were you satisfied with the allocated treatment at the start of the program?", (b) "Has your opinion about the treatment changed during the program?", and (c) "How satisfied are you now about the treatment that was given to you?"

\section{Short- and long-term follow-up}

Beside a baseline measurement, follow-up data are gathered at two short-term intervals and two long-term intervals. Short-term follow-up measurements are at 5 and 10 weeks after randomization. Long-term follow-up measurements are at 6 months and one year after the end of the intervention, respectively. At every test moment, participants fill in a compound questionnaire on a standalone PC, and they undergo an isometric back strength measurement on the lower back machine. The content of the questionnaire varies per test moment: parts of the questionnaire referring to potential prognostic variables (job characteristics, overall health, and physical activity) are only displayed at baseline, and 
at 6 and 12 months of follow-up. Figure 1 presents a flow chart of the different study phases.

\section{Blinding}

Double blinding or placebo control is virtually impossible in trials that involve treatment modalities like the ones used in this study because both patient and provider are inevitably aware of the content of the treatment. Because our physiotherapists are aware of which treatment they provide, there is always the possibility that they may inadvertently convey different expectations to the patients in each treatment program. This could enhance nonspecific effects in either of the two groups. Therefore, as mentioned, beliefs and attitude of the physiotherapists towards back treatment in general are evaluated at baseline, as well as at the end of the study period.

Another limitation of trials comparing a relatively new treatment to the usual care is the risk of a potential nocebo effect; i.e., patients might feel disappointed after being allocated to the standard therapy. To minimize this effect, both programs are introduced to the patient as potentially equally effective treatments in restoring back function, with the relative efficacy of both programs as the main focus of the study. Moreover, at baseline patients are informed about the opportunity they have to continue with a treatment modality of their choice (e.g., our training device) after finishing the treatment period. Patient satisfaction with the allocated treatment is measured directly after the treatment period.

Other efforts to achieve a certain level of blinding within patients, physiotherapists, and data researchers, are:

- low back strength training and measurement are done as much as possible by two different physiotherapists;

- an independent data manager collects data from all study locations, and recodes patients and locations to unique codes before handing the database to the researchers. 


\section{Statistics}

\section{Sample size estimates}

We attempt to enroll 200 patients at the four military health centers, i.e., 100 patients per treatment group. According to power calculations $(\alpha=0.05$ and $1-b=80 \%$ ), this sample size is sufficient to detect a $20 \%$ difference in our primary outcome measure Global Perceived Effect, between the BS and UC program. In our beliefs, a $20 \%$ difference reflects a clinical relevant change in health status.

\section{Data analysis}

Statistical analysis will be performed according to the intention-to-treat principle; i.e., patients will be analyzed in the treatment group to which they were randomly allocated. In addition, a per-protocol analysis will be performed, in which only patients with no major protocol deviations will be analyzed. Comparing these analyses with those of the intention-to-treat analysis will indicate, to what extend protocol deviations and lack of compliance might have biased the results.

In our analyses, we will compare the size of the effect, if any, of isolated back strengthening and of usual care for the low back on our primary and secondary outcome variables. Further analysis will determine whether several potential prognostic variables will influence the magnitude of the treatment responses, and if subsets of patients can be distinguished that can be indicated as good-/ bad-responders to our specific back training. Demographic and clinical characteristics, as well as baseline outcome measures will be summarized by descriptive statistics. Longitudinal multilevel analyses will be used to examine differences in all continuous outcome measures at 5 and 10 weeks after randomization, and 6 and 12 months of follow-up.

\section{Supervision of the centers}

In order to obtain full commitment from the participating military health centers, as well as to make sure that every center uses the study protocols in the same way, we organize several feedback and feedforward sessions with all participating physiotherapists. In these sessions, we explain various aspects of the trial 
design, give instructions on the test and training protocols, and answer remaining questions.

After these sessions, we install the required equipment (test/training machine, soft- and hardware) at the four locations. Each center has a practice period of about 8 weeks to become familiar with the instruments, logistics and protocols, by means of ad hoc training and test sessions with nonparticipating (regular) back patients. Our researchers join these sessions and, if necessary, give correcting feedback during and after a test or training. After this practice period, each location officially starts with the study. Once every two weeks, our researchers visit the centers to monitor progress in recruitment and data handling, and to observe the execution of test and training sessions.

\section{Discussion}

This article describes the rationale and design of a multicenter randomized controlled trial in which the efficacy of a specific type of lumbar extension training and usual care are compared in patients with nonspecific LBP longer than 4 weeks. A substantial number of trials have been conducted that included lumbar extension training in low back pain patients $4,7,11,12,32,33$. So far, only the study by Risch et $\mathrm{al}^{11}$ has emphasized a minimal intervention approach comparable to ours, which was, however, conducted in a nonrandomized study design.

Our population at risk can be seen as a selected population of mainly male employees, who work in a dynamic organization with a strong culture of physical fitness. We realize that this selection might limit the external validity of the outcomes of this study. However, results of the trial may be extrapolated to other working populations with a more-than-average degree of physical straining job activities (e.g., policemen and firemen on duty or construction workers). Besides, despite the "fit-and-healthy" image that soldiers have in our society, a large health survey among a cross-section of male military personnel of 30-40 years showed no favorable scores on several cardiovascular and fitness parameters in comparison to other populations of Dutch men ${ }^{34,35}$. In this perspective, the RNLA - with over 30,000 military and civilian employees a major profes- 
sional organization in the Netherlands - seems to be a good reflection of Dutch society in general with regard to general health parameters.

Moreover, for reasons of homogeneity it might be even an advantage to only have a study population of working men with (probably) moderate low back trouble in this study.

We hope with this trial to give greater insight to caregivers within and outside the RNLA on treatment options for workers in the sub-acute or chronic phase of their LBP. If, for instance, our minimal intervention approach is equally or more effective than the usual care, medical decision makers may consider implementing this treatment modality in the daily practice of the physiotherapist, by weighing the costs (e.g., price and depreciation costs of materials) and benefits (e.g., reduction of treatment time).

\section{Competing interests}

The authors declare that they have no competing interests.

Acknowledgements: The authors gratefully acknowledge all participating military general practitioners and physiotherapists for their work on location. We would like to thank Peter de Putter and Wim Veenman for their efforts in the development and maintenance of the ALBATROS software program, as well as in the management of all data. Finally we would like to thank Colonel René Roelofs, MD, Surgeon General RNLA, and Colonel Kees IJzerman, MD, Director Occupational Health \& Safety Service RNLA, for authorizing this multicenter study. 


\section{References}

1. Mooney V, Kenney $\mathrm{K}$, Leggett $\mathrm{S}$, Holmes B: Relationship of lumbar strength in shipyard workers to workplace injury claims. Spine 1996, 21:2001-5

2. van Tulder $M$, Furlan $A$, Bombardier $C$ et al. Updated method guidelines for systematic reviews in the cochrane collaboration back review group. Spine 2003;28:1290-9.

3. Mior S. Exercise in the treatment of chronic pain. Clin.J.Pain 2001;17:S77-S85.

4. Bronfort $\mathrm{G}$, Goldsmith $\mathrm{CH}$, Nelson $\mathrm{CF}$, et al. Trunk exercise comined with spinal manipulative or NSAID therapy for chronic low back pain: A randomized, observer-blinded clinical trial. J Manipulative Physiol Ther 1996;19(9):570-82.

5. Mannion AF, Muntener M, Taimela S, Dvorak J. A randomized clinical trial of three active therapies for chronic low back pain. Spine 1999;24:2435-48.

6. Hansen FR, Bendix T, Skov P, Jensen CV, Kristensen JH, Krohn L, Schioeler H. Intensive, dynamic back-muscle exercises, conventional physiotherapy, or placebo-control treatment of low-back pain: A randomized, obser verblind trial. Spine 1993;18:98-108.

7. Leggett S, Mooney V, Matheson LN, Nelson B, Dreisinger T, Van Zytveld J, Vie L. Restorative exercise for clinical low back pain: A prospective two-center study with 1-year follow-up. Spine 1999;24:889-98.

8. Miltner $\mathrm{O}$, Wirtz DC, Siebert $\mathrm{CH}$. [Strengthening lumbar extensors--therapy of chronic back pain-an overview and meta-analysis]. Z Orthop Ihre Grenzgeb 2001;139:287-93.

9. Carpenter DM, Nelson BW: Low back strengthening for the prevention and treatment of low back pain. Med Sci Sports Exerc 1999, 31:18-24

10. SV Risch, NK Norvell, ML Pollock, ED Risch, H Langer, M Fulton, JE Graves, SH Leggett: Lumbar strengthening in chronic low back pain patients. Physiologic and psychological benefits. Spine 1993, 18:232-8.

11. Nelson B, O'Reilly E, Miller M, Hogan M, Wegner JA, Kelly C: The clinical effects of intensive, specific exercise on chronic low back pain:a controlled study of 895 consecutive patients with 1-year follow up. Orthopedics 1995, 18:971-81

12. Mooney $V$, Kron M, Rummerfield P: The effect of workplace based strengthening on low back injury rates: a case study in the mining industry. J Ocup Rehabil 1995,5:157-167.

13. Tucci JT, Carpenter DM, Pollock ML, Graves JE, Leggett SH: Effect of reduced frequency of training and detraining on lumbar extension strength. Spine 1992, 17:1497-501.

14. Helmhout PH, Harts CC, Staal JB, Candel MJ, de Bie RA: Comparison of a high-intensity and a low-intensity lumbar extensor training program as minimal intervention treatment in low back pain: a randomized trial. Eur Spine J 2004;13:537-47.

15. Karasek R, Brisson C, Kawakami N, Houtman I, Bongers P, Amick B: The Job Content Questionnaire (JCQ): an instrument for internationally comparative assessments of psychosocial job characteristics. J Occup Health Psychol 1998, 3:322-55.

16. Hildebrandt VH, Bongers PM, van Dijk FJ, Kemper HC, Dul J: Dutch Musculoskeletal Questionnaire: description and basic qualities. Ergonomics 2001, 44:1038-55.

17. Ware JE, Sherbourne CD (1992) The MOS 36 item Short Form Health Survey (SF-36). Med Care 30:473-483

18. Wendel-Vos GC, Schuit AJ, Saris WH, et al. Reproducibility and relative validity of the short questionnaire to assess health-enhancing physical activity. J Clin Epidemiol 2003;56(12):1163-9. 
19. Ostelo RW, Stomp-van den Berg SG, Vlaeyen JW, et al. Health care provider's attitudes and beliefs towards chronic low back pain: the development of a questionnaire. Man Ther 2003;8:214-22.

20. Beurskens AJ, de Vet HC, Koke AJ. Responsiveness of functional status in low back pain: A comparison of different instruments. Pain 1996;65:71-6.

21. Beurskens AJ, de Vet HC, Koke AJ, Lindeman E, van der Heijden GJ, Regtop W, Knipschild PG. A patient-specific approach for measuring functional status in low back pain. J Manipulative Physiol Ther 1999;22:144-8.

22. Gommans I, Koes B, Van Tulder M. Validiteit en responsiviteit Nederlandse Roland Disability Questionnaire: Vragenlijst naar functionele status bij patiënten met lage rugpijn. Ned Tijdschr Fysiother 1997;2:28-33.

23. Roland M, Morris R. A study of the natural history of back pain. Part I: development of a reliable and sensitive measure of disability in low-back pain. Spine 1983;8:141-4.

24. Stratford PW, Binkley JM, Riddle DL, Guyatt GH (1998): Sensitivity to change of the Roland- Morris Back Pain Questionnaire: part 1. Physical Therapy 78; 1186-1196.

25. Stratford PW, Binkley J, Solomon P, Gill C, Finch E: Assessing change over time in patients with low back pain. Phys Ther 1994, 74:528-33.

26. Miller R, Kori S, Todd D: The Tampa Scale. In: Book The Tampa Scale (eds.). City,1991.

27. Goubert L, Crombez G, Vlaeyen J, van Damme S, van den Broeck A, van Houdenhove B: De Tampa schaal voor kinesiofobie. Psychometrische karakteristieken en normering. Gedrag Gezond 2000, 28:54-62.

28. Vlaeyen JW, Kole-Snijders AM, Boeren RG, van Eek H: Fear of movement/(re)injury in chronic low back pain and its relation to behavioral performance.Pain 1995, 62:363-72.

29. D Goldberg, P Williams: A user's guide to the general health questionnaire. Windsor: NFER-Nelson; 1988.

30. Koeter M, Ormel J: General Health Questionnaire. Handleiding Nederlandse bewerking. Lisse: Swets and Zeitlinger; 1991

31. Cardol M, de Haan RJ, van den Bos GA, de Jong BA, de Groot IJ: The development of a handicap assessment questionnaire: the Impact on Participation and Autonomy (IPA). Clin Rehabil 1999, 13:411-9.

32. Manniche C, Lundberg E, Christensen I, Bentzen L, Hesselsoe G: Intensive dynamic back exercises for chronic low back pain: a clinical trial. Pain 1991, 47:53-63.

33. Kankaanpaa M, Taimela S, Airaksinen O, Hanninen O: The efficacy of active rehabilitation in chronic low back pain. Effect on pain intensity, self-experienced disability, and lumbar fatigability. Spine 1999, 24:1034-42.

34. Brussaard J, Meuling W, den Breijen J: Hernieuwde peiling fitheid en gezondheid: deel 1. In: Book Hernieuwde peiling fitheid en gezondheid: deel 1 (eds.). City, 1997.

35. Brussaard J, Meuling W, den Breijen J: Hernieuwde peiling fitheid en gezondheid: deel 2. In: Book Hernieuwde peiling fitheid en gezond 



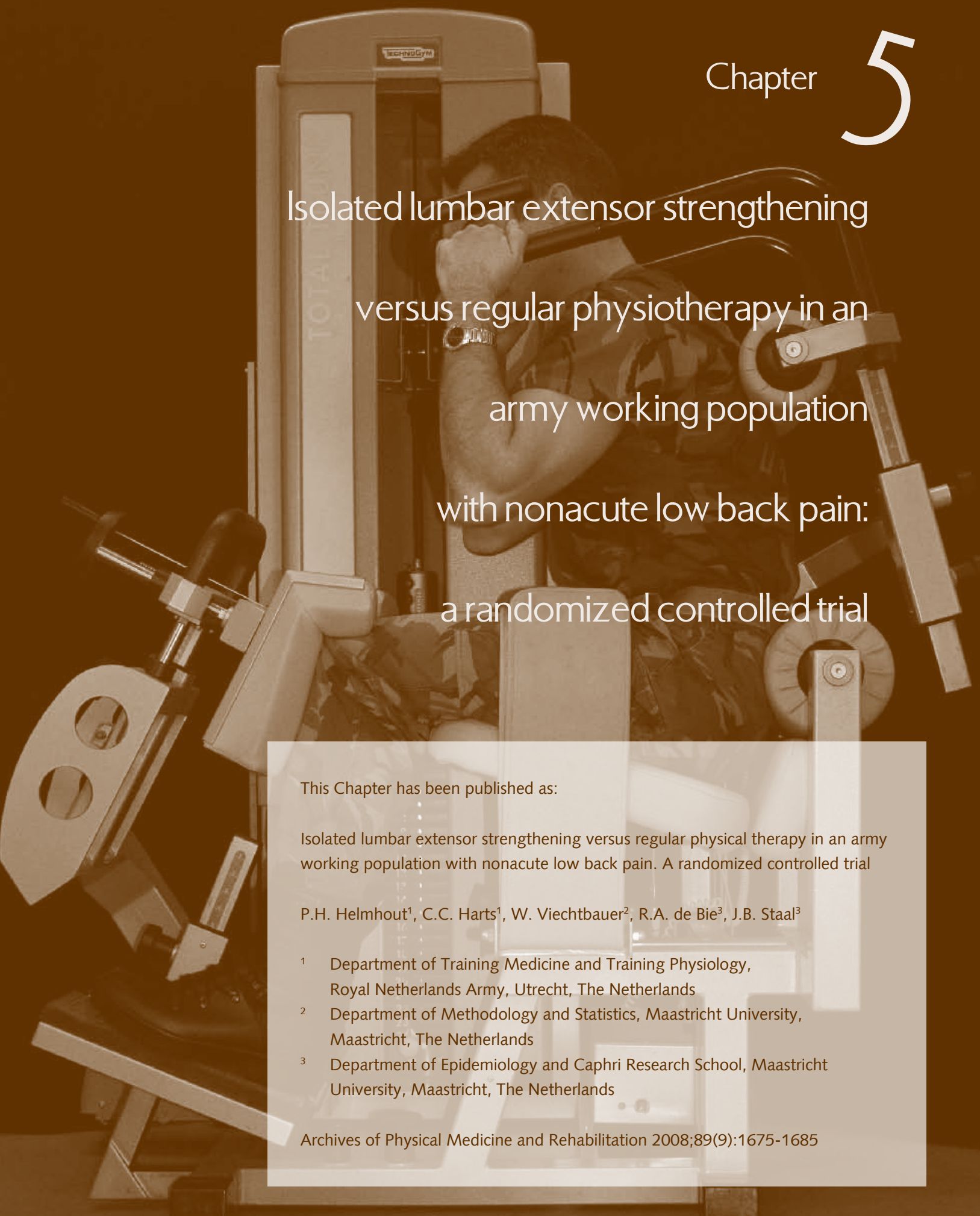




\section{Abstract}

Objective: To evaluate the effectiveness of specific lumbar extensor training compared with regular physical therapy (PT) in workers with nonacute nonspecific low back pain (LBP).

Design: Multicentered randomized controlled trial with 1-year follow-up.

Setting: PT department in (military primary care) health centers

Participants: A total of 129 predominantly male soldiers with 4 weeks or more of low back complaints who were referred by the health center' general practitioner to physiotherapy (mean age, $35.9 \pm 10.8$ years; range $20-56$ years), of which 127 randomized participants were included in the analyses. One patient withdrew as a result of adverse effects during treatment.

Interventions: Participants were assigned to one of 2 treatment programs: (1) a 10-week device-supported isolated lumbar extension training, twice a week, or (2) regular PT, mainly consisting of exercise therapy and aerobic activities. Main Outcome Measures: Functional status (Roland-Morris Disability Questionnaire, Patient-specific Functional Scale) and global perceived effect were assessed in the short-term (5, 10 weeks) and long-term (1/2,1 year).

Results: Both groups showed a favorable development in main outcomes over time: short-term improvements (after 10 weeks of treatment) remained stable or even slightly increased throughout the 1-year follow-up. No differences between the 2 groups were shown for any of the outcome measures, at any time. Conclusions: Consistent with prior evidence, specific back strengthening does not seem to offer incremental benefits in LBP management when compared to regular PT care that mainly consists of general exercise therapy.

List of abbreviations: $G P=$ general practitioner, $G P E=$ global perceived effect, $L B P=10 \mathrm{~W}$ back pain, 1-RM = one-repetition maximum, PSFS = Patient-Specific Functional Scale, $P T=$ physiotherapist,$R M D Q=$ Roland-Morris Disability Questionnaire, $T S K=$ Tampa Scale for Kinesiophobia 


\section{Introduction}

Low back disorders have become a major medical and socioeconomic problem among working populations in industrialized countries. ${ }^{1,2}$ Recent-onset LBP often recurs intermittently, sometimes resulting in persistent disabling symptoms. Once chronic, the back pain condition can significantly impact an injured worker's quality of life and productivity, ${ }^{3,4}$ generating substantial costs related to medical service utilization, sick leave compensation, disability pension, and replacement costs. ${ }^{5-8}$

The Royal Netherlands Army is a major professional organization in the Nether lands, employing approximately 25,000 military and civilian personnel. The diverse workforce is involved in a wide range of jobs, making the Dutch army comparable to other organizations with a high degree of physical straining job activities, such as the police force, fire brigade, health service or the construction industry. Military occupations that involve heavy physical training programs (e.g., Special Forces; Infantrymen) and/or frequent manual material handling tasks (e.g., Construction Equipment Operators; Heavy Vehicle Mechanics) are associated with higher-than-average risk of disability. ${ }^{9}$ These types of jobs may expose workers to occupational risk factors that have been specifically associated with low back disability, such as repetitive lifting, frequent bending and twisting, pushing and pulling, and awkward body postures. ${ }^{10-12}$

Back-related disorders represent one of the most prevalent sources of disability in personnel of the Dutch army. (Helmhout, unpublished data, 1998) Acute LBP is the primary reason for Dutch soldiers to visit the general practitioner at a military health center. Chronic nonspecific LBP, defined as having complaints for at least 12 weeks, is one of the 3 most diagnosed disorders during general practitioners (GP) consulting hours; managing care-seekers with LBP takes up to $15 \%$ of the military company doctor's weekly consulting hours time (Helmhout, unpublished data, 2003).

Since the 1990s, "passive" forms of PT treatments such as massage and electrophysical treatments have become less popular, while exercise therapy has grown 
in popularity. Recent reviews conclude that exercise is an effective conservative treatment for persistent LBP in terms of restored function, reduction of sick leave and alleviation of pain, though improvements in outcomes such as pain and functional limitations are generally small and brief. ${ }^{13-18}$ Efficacy studies on exercise therapy generally show a lack of treatment specificity of various exercise modalities such as aerobic exercise, muscle strengthening or stabilization. ${ }^{19-21}$ Recent reviews by Slade et $\mathrm{al}^{13}$ and Hayden et $\mathrm{al}^{14}$ indicate that the optimal type of exercise for LBP may not be known because the effects of specific exercises have not been systematically assessed. Another explanation for the nonspecific effects of exercise therapy could be the heterogeneity in demographic and clinical characteristics of many study populations..$^{22-23}$

For the last ten years, clinical researchers of the Royal Netherlands Army have studied the potential of physical training modalities in preventing and alleviating nonspecific LBP in their working population. This research has led to a minimal intervention program existing of no more than approximately 10 minutes of specific training of the isolated lumbar extensors on a training device, while adhering to sports medicine principles. An extensive elaboration on the rationale for the use of this approach has been published elsewhere. ${ }^{24}$ In short, physical fitness can be seen as a critical aspect of military prepardeness and an inherent part of military service. The necessary high levels of physical fitness needed by armed forces can only be achieved through systematic, repeated, general, and task-specific physical exercise. From the initial military education, soldiers are exposed to unit physical fitness training programs, including progressive resistance training on exercise machines. In our experience, this repeated occupational exposure to physical exercise shapes the beliefs, attitudes, and coping behaviour of, for instance, a soldier with chronic pain towards a strong physical orientation. In an earlier study we found that service members with chronic LBP perceived their health problems as mainly physically disabling. ${ }^{25}$ Interventions such as isolated lumbar extensor training, which predominantly focusses on physical reconditioning using devices, may therefore be especially appropriate for our target population. The limited dimensions of our exercise program are expected to be highly appreciated in a military setting of constant change. 
Earlier studies on isolated lumbar extensor training suggest that this type of progressive exercise can be of value for back pain management. ${ }^{26-28}$ Overall, promising results were reported regarding strength improvements and pain relief. However, several methodological shortcomings hinder solid interpretation of these findings. Most encountered problems were small population size, lack of randomization and inadequate or missing control groups. In a review, Miltner et al ${ }^{29}$ have stressed the need for randomized trials to further assess the efficacy of isolated lumbar extensor exercise in LBP management.

Literature from the early 1990s showed some evidence for a dose-response effect of exercise for chronic LBP. ${ }^{30-31}$ In 2 previous trials at our department in Utrecht, The Netherlands, we compared the efficacy of a high-intensity versus low-intensity training protocol for the lumbar extensors, in a group of workers with nonspecific LBP. Total intervention time of both minimal intervention programs was limited to 10 to 14 sessions of approximately 10 minutes each, over a period of 12 weeks ( $1{ }^{\text {st }}$ trial) or 8 weeks ( ${ }^{\text {nd }}$ trial). In the first trial, ${ }_{1}^{25}$ we were unable to demonstrate that either of the 2 training programs was superior in alleviating back complaints in a group of 81 workers. The magnitude of the improvements in back function found in this study were in line with those reported in studies that used more extended multidisciplinary exercise programs. Moreover, the results of our first trial indicated that some individuals with LBP might benefit more from an aggressive approach, showing a trend towards a higher self-assessed percentage decrease in complaints directly after the minimal intervention treatment, as well as a higher compliance to the treatment and a higher willingness to participate in physical exercise on the longer term. The second study, ${ }^{32}$ which also included a waiting-list group, showed that for most outcome measures the point estimates were slightly in favor of the 2 exercise groups compared to the waiting-list group. Effects were small and mostly not statistically significant, possibly as a result of the low number of participants (65 workers distributed over 3 intervention arms).

On the basis of our previous work, we concluded that further exploration was needed into the optimal type, intensity, and frequency of lumbar extensor training in LBP. Other factors like the mediating role of pain-related fear, degree 
of physical activity, and/or job characteristics could be explored. We therefore set up a randomized controlled trial in which various military health centers participated, in an attempt to overcome previous problems of underpowering. Moreover, we slightly modified our test and training protocol based on previous experience (e.g., 10-week program; two times a week; one training set). Since the population under study (soldiers visiting a military GP for their back trouble) generally had more acute symptoms than our previous study populations, we extended our inclusion criterion from 12 to 4 weeks of LBP. This article reports the results of the multicenter trial, in which the effectiveness of isolated lumbar extensor training compared with regular PT is evaluated in workers with nonacute nonspecific LBP. An extensive description of the design of the trial (registered as ISRCTN19334317) was published in 2004.24

\section{Methods}

\section{Recruitment and randomization of study group}

The source population consists of military employees of the Royal Netherlands Army $(n=23,000)$. Participants were recruited in 6 military (primary care) health centers during regular GP consulting hours. Patient recruitment commenced in April 2002 and continued until December 2005. Inclusion criteria were: military employees of the Dutch army between the age of 18 and 54 years; at least 4 weeks of continuous or recurrent (at least 3 times a week) episodes of LBP; pain localized between posterior iliac crests and angulus inferior scapulae, with or without radiation in the legs; availability in duty time to visit the local military health center 2 times a week during 10 consecutive weeks, with no more than 2 sessions of absence because of job-related activities (e.g., military exercise; course; leave), and willingness to abandon other treatment interventions for the lower back during the intervention period. Exclusion criteria were: spinal surgery in the last 2 years; specific treatment for LBP in the last 4 weeks (e.g., PT; manual therapy); severe LBP which hinders in performing maximal isometric strength efforts; specific LBP, defined as herniated disk, ankylosing spondylitis, spondylolisthesis or other relevant neurological diseases. 
After physical examination by the physician, eligible patients received information about the study and a referral to the military physiotherapist. In 2 PT consults, participants underwent a trial measurement and a baseline measurement, respectively, consisting of a compound questionnaire and an isometric back strength test. The main purpose of the trial measurement was that participants could get accustomed to the testing procedure, in particular the isometric back strength test. In a previous test-retest reliability study, we found that reproducibility of this test was lower between an initial and second measurement, than between a second and third measurement. (C. Harts, P.Helmhout, unpublished data, 1998) Written informed consent was obtained from all participants. At the third visit, patients were randomized into 2 groups: an intervention group receiving an isolated lumbar extensor training program, and a reference group receiving regular PT. Randomization was done by means of a computer-generated table of random numbers per study center, using a block size of 10 . Prestratification was applied for the duration of the back complaints, with a cutoff point of 12 weeks, with suspicion of duration of complaints to influence the individual response to the program. Randomization was concealed by means of a computer program. After the name and military registration number of the patient were entered by the physiotherapist, the program provided the allocated treatment. Directly after randomization, the first treatment session started. The study protocol was approved by the Medical Ethics Committee of the Netherlands Central Military Hospital, Utrecht.

\section{Study interventions}

\section{Lumbar extensor strength training program}

The lumbar extensor training program was a highly standardized, progressive resistance training of the isolated lumbar extensor muscle groups, covering 10 weeks of training. The program was provided by PTs from the military health center. The program included 14 training sessions twice a week, and 3 isometric back strength tests (in week 1, 5, and 10). The program aimed at both strength and endurance gain of the back muscles, in that the protocol allowed 15 to 20 repetitions every session on the lower back machine, which is equivalent to approximately $50 \%$ and $70 \%$ of the one-repetition maximum (1-RM) respec- 
tively. ${ }^{33}$ If the subject was able to perform more than 20 repetitions of slow and controlled back extension ( $2 \mathrm{~s}$ ) and flexion (4 s) movements, $2.5 \mathrm{~kg}$ weight was added in the next training session. Vice versa, if the subject was unable to perform 15 repetitions, the training load was lowered with $2.5 \mathrm{~kg}$ next time. This training protocol is based on both existing protocols $\mathrm{s}^{34-37}$ and our own clinical experiences. A more extensive description of the training protocol can be found elsewhere. ${ }^{24}$ Training sessions were carried out on a Total Trunk Rehab machine. This lower back machine is equipped with a knee-lock system and a thigh-restraining belt to immobilize both hips and thighs, allowing the participant only to move the isolated lower back. All training sessions were conducted as much as possible by the same physiotherapist, who paid special attention to the execution of the training in terms of execution (hollowing and flattening of the lumbar lordosis), pace (relatively slow), and movement (full range). The weight load used and the number of repetitions completed during each training session were recorded. Patients were not allowed to undergo cotreatments beside the Lumbar Exensor Strength Training Program during the treatment period.

\section{Regular PT program}

Subjects allocated to the regular PT program received regular PT for their lower back for 10 weeks, or less when the patient was free of complaints. The therapy was provided by physiotherapists from the military health center. Based on the physiotherapists' judgment, this could include hands-on treatment (e.g., passive mobilizing and pain-cushioning techniques; manual therapy) and/or hands-off treatment (e.g., exercise therapy; individual education and instruction on the back function). In the Dutch army, active therapy forms are favored. To increase the contrast between both intervention programs, physiotherapists were not allowed to use the lower back machine in their usual care. Patients were also not allowed to undergo cotreatments beside the regular PT program during the treatment period, nor exercise on equipment that mimicked the specific components of the lower back machine. Therapeutic activities in every therapy session as well as the number of sessions were written down on a form. 


\section{Outcome measurements}

In this study we have chosen low-back specific functional status as our primary outcome, which was measured with the validated Dutch 24 -item version of the widely used RMDQ. ${ }^{38-39}$ Patient-specific functional status was measured by the PSFS, in which patients selected at baseline the 3 most important ADL-activities that were hampered by their back pain, and rated them on a 100-mm visual analogue scale at each test moment. ${ }^{40} \mathrm{GPE}$ was measured by self-assessment on a 7-point scale ( $1=$ completely recovered, $2=$ much improved, $3=$ slightly improved, $4=$ no change, $5=$ =slightly worsened, $6=$ much worsened, $7=$ vastly worsened) ${ }^{41}$

The following demographic variables were registered during the intake: age, height, weight, duration of current back complaints, earlier episodes of LBP, pain radiation, change of work and work absenteeism because of LBP in the last year.

As a potential effect modifier, fear of movement or re-injury was measured using the Dutch version of the TSK, a 17-item scale to obtain a score for the extent to which a back patient fears (new) physical damage as a result of physical activity. ${ }^{42-44}$ Moreover, individual back extension strength progression was evaluated using repeated isometric measurements on the lower back training and testing machine. A detailed description of these measurements can be found elsewhere. ${ }^{24}$

Patient satisfaction was measured at the end of the treatment program by two 3-level items and one 5-level item in which the degree of satisfaction with the allocated treatment was assessed:

a. Were you satisfied with the allocated treatment at the start of the program?

b. Has your opinion about the treatment changed during the program?

c. How satisfied are you now about the treatment that was given to you?

\section{Short- and long-term follow-up}

Follow-up data were collected at 2 short-term intervals at 5 and 10 weeks after randomization, respectively, and 2 long-term intervals at 6 months and 1 year after the end of the intervention, respectively. At every test moment, partici- 
pants filled in a compound questionnaire on a stand-alone personal computer, and they underwent an back strength measurement on the lower back machine.

\section{Blinding}

Blinding of patients was not possible because of the nature of the interventions. However, in an attempt to minimize potential novelty effects (i.e., allocation to the new lumbar extensor strength training program treatment causes temporary improvements in perceived complaints) ${ }^{45}$, both lumbar extensor strength training and regular PT were introduced to the patient as potentially equally effective treatments in restoring back function, with the relative efficacy of both programs as the main focus of the study. Patients were informed at baseline about the opportunity they had to continue with a treatment modality of their choice, including our training device, after finishing the treatment period. Patient satisfaction with the allocated treatment was measured directly after the treatment period. Moreover, training sessions and strength measurements were as much as possible administered by different physiotherapists. Knowledge on individual training progression could not influence the process of measurement and vice versa. An independent data manager, who had not been involved in the study thus far, collected data from all study locations, and recoded patients and locations to unique codes before handing the database to the researchers.

\section{Statistical Analysis}

We intended to include 100 participants a treatment arm, which should be sufficient to detect a 2-point difference in our primary outcome variable RMDQ between both intervention groups. ${ }^{46}$ According to Resnik et $\mathrm{al}_{1}{ }^{47}$ a 2-point to 3 -point change can be considered clinically important in RMDQ values that are less than 9 points at baseline. For the calculation of the sample size, we further used an SD of 4.8 (based on our first trial), a dropout rate of approximately $10 \%$, an $\alpha$ of .05 and a power of $80 \%$.

Differences between lumbar extensor strength training and regular PT in the development of the outcome measures RMDQ, PSFS, and GPE were separately described for the study treatment period and the follow-up period, respectively. We performed longitudinal linear regression analysis for repeated measurements 
using mixed effects models. Analyses were carried out with the nlme library ${ }^{48}$ under $\mathrm{R}$, version 2.5.0. ${ }^{49}$

To avoid model overfitting and multiple testing, ${ }^{50}$ models for the 3 dependent variables were chosen a priori on the basis of theoretical considerations and prior empirical findings, such as the following: baseline differences were not expected because of randomization; trends over time were assumed to be linear; slopes were allowed to differ between the treatment phase and follow-up phase, as well as between both treatment groups; and random intercepts and slopes were modelled, with treatment location as a fixed effect.

Before fitting the a priori models, a so-called unconditional means model and unconditional growth model were both fitted to the data for all 3 dependent variables. The unconditional means model allowed us to assess how much variability existed at the between-person and within-person level. The unconditional growth model modeled the trend over time without including predictors that might account for differences in the intercepts and slopes across treatment groups, locations, or subjects. Next, the a priori models were fitted by including the treatment group and location variables, to account for some of the variability in person-specific intercepts and slopes of the unconditional growth model.

In the models for RMDQ and PSFS, the main (null) hypothesis to be tested was as follows: the average slope of the linear trend in the treatment phase and the follow-up phase, respectively, is the same for lumbar extensor strength training and regular PT. In the model for GPE, the main (null) hypotheses to be tested were as follows: (1) lumbar extensor strength training and regular PT do not differ with respect to the average at the beginning of the follow-up phase, and (2) the average slope of the linear trend in the follow-up phase is the same for lumbar extensor strength training and regular PT. Finally, an additional effect modifier model was fitted by including 2 potential effect modifiers: duration of current complaints ${ }^{11,51}$ and baseline TSK score ${ }^{52-54}$. The values of these potential effect modifiers were adjusted prior to their inclusion in the model by subtracting the median value from each individual value in order to give a more natural interpretation to the model parameters. 


\section{Results}

Figure 1 shows the progress through the different phases of the trial. We enrolled 127 subjects into the study before ending our recruitment activities. Mainly practical reasons underlay the decision to end the recruitment before meeting the pre-specified sample size calculation, such as a declining recruitment rate $(<3$ enrollments per month towards the end), a long recruitment period (nearly $4 y$ ), and the fact that one of the major military health centers was about to close.

Our final database consisted of 5 repeated measurements on 127 predominantly male subjects with moderate chronic back complaints, who were clustered in 6 different treatment locations (health centers) and randomly assigned to the 2 treatment groups lumbar extensor strength training or regular PT within each location. Table 1 shows baseline characteristics of the subjects in both treatment arms. The lumbar extensor strength training group contained relatively more subjects with chronic back complaints (>3 months) compared with the regular PT group. One subject was randomized in regular PT but incorrectly received lumbar extensor strength training; he was analyzed in the regular PT group, according to the intention-to-treat principle. Because of planning difficulties at the health centres, our trial showed some variability between subjects in time of measurement, though not significantly different between locations: the 5-week intermediate assessment was planned, on average, in week $6(S D=1.5$; range, 4-13); the 10-week post-treatment assessment in week 12 (SD=2.2; range, 818); the 36-week short-term follow-up in week 39 (SD=7.0; range, 25-60); and the 62-week long-term follow-up in week 70 ( $S D=12.9$; range, 50-100). For this reason, the actual individual measurement week was included as a timing variable in the analyses.

The average number of treatment sessions $( \pm S D)$ in the lumbar extensor strength training program was $13.9 \pm 3.7$, and in the regular PT program $10.8 \pm 4.7$. The participating PTs reported mean intervention times per treatment session of 10 to 15 minutes in the lumbar extensor strength training group, and 25 to 30 minutes in the regular PT group. 


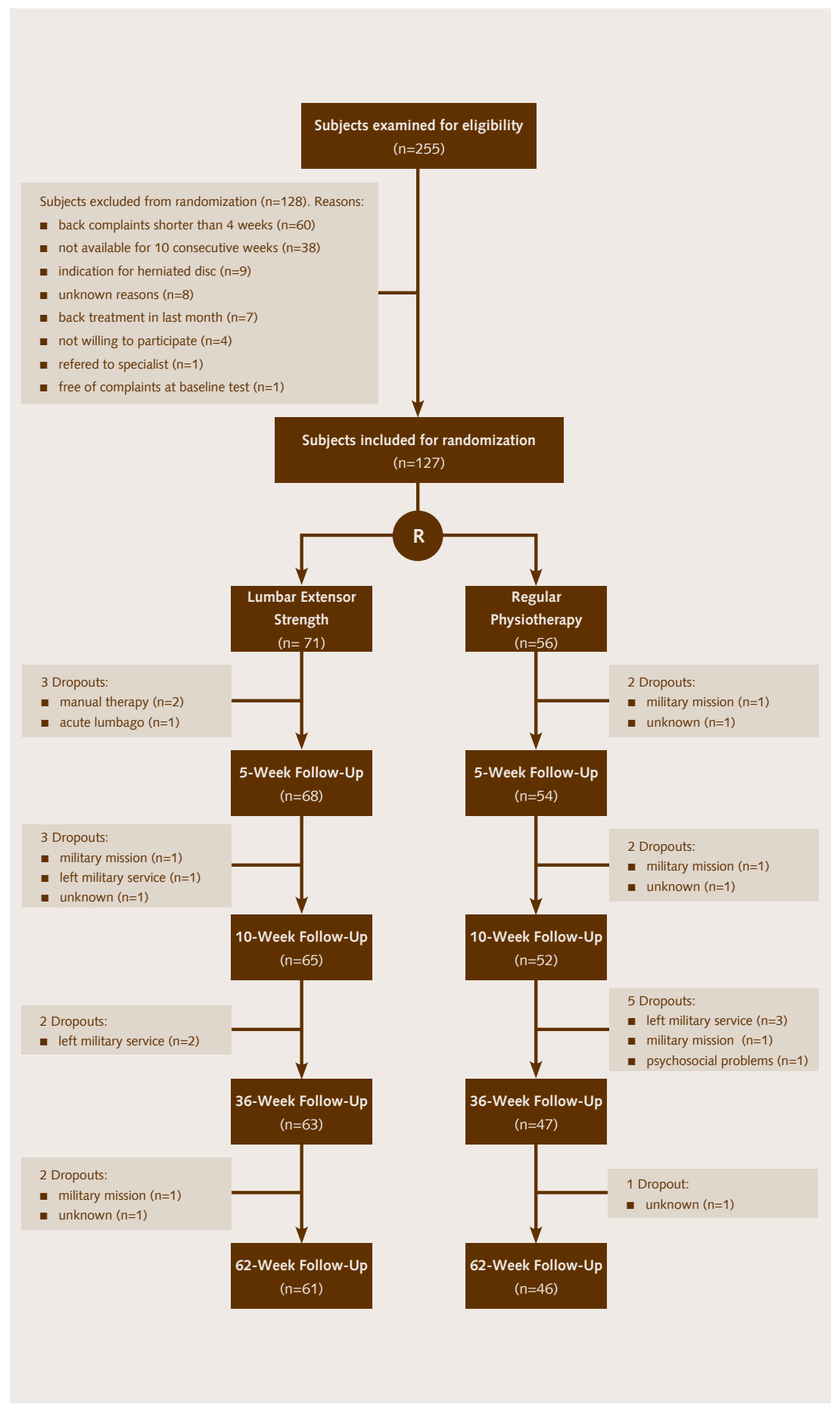

Figure 1. Flow of participants through the trial. 
Table 1. Baseline demographic and clinical characteristics and baseline outcome measures of trial participants in both treatment groups. Mean values (SD; range) are presented, unless otherwise stated.

\begin{tabular}{|c|c|c|}
\hline & $\begin{array}{l}\text { Lumbar Extensor } \\
\text { Strength } \\
(n=71)\end{array}$ & $\begin{array}{c}\text { Regular } \\
\text { Physiotherapy } \\
(n=56)\end{array}$ \\
\hline \multicolumn{3}{|l|}{ Demographic Characteristics } \\
\hline $\begin{array}{l}\text { Age, in years } \\
\text { Height, in } \mathrm{cm} \\
\text { Weight, in } \mathrm{kg} \\
\text { Male sexe, in \% }\end{array}$ & $\begin{array}{l}37(11 ; 20-53) \\
183(8 ; 157-196) \\
85(12 ; 50-116) \\
97\end{array}$ & $\begin{array}{l}35(11 ; 21-56) \\
182(7 ; 165-198) \\
86(11 ; 65-108) \\
96\end{array}$ \\
\hline \multicolumn{3}{|l|}{ Clinical Characteristics } \\
\hline $\begin{array}{l}\text { Duration current back complaints, in } \% \\
\qquad \begin{array}{l}<\text { weeks } \\
4-6 \text { weeks } \\
6-12 \text { weeks } \\
3-6 \text { months } \\
6-12 \text { months } \\
\geq 1 \text { year }\end{array}\end{array}$ & $\begin{array}{l}(n=59) \\
0 \\
8 \\
20 \\
20 \\
15 \\
36\end{array}$ & $\begin{array}{l}(n=44) \\
2 \\
16 \\
27 \\
9 \\
7 \\
39\end{array}$ \\
\hline $\begin{array}{l}\text { Earlier episode(s) of back complaints, in \% } \\
\text { Pain radiation in legs, in \% } \\
\text { Change of work due to back complaints, in \% } \\
\text { Work absenteism in last year due to back, in \% }\end{array}$ & $\begin{array}{l}76(n=64) \\
10(n=63) \\
0(n=48) \\
10(n=48)\end{array}$ & $\begin{array}{l}74(n=51) \\
10(n=51) \\
0(n=38) \\
8(n=38)\end{array}$ \\
\hline \multicolumn{3}{|l|}{ Outcome Measures } \\
\hline $\begin{array}{l}\text { RDQ Score, range } 0-24 \text { points } \\
\text { PFS Score, range } 0-300 \text { points } \\
\text { TSK Score, range } 17-68 \text { points } \\
\text { NMT, in Nm }\end{array}$ & $\begin{array}{l}8.3(4.8 ; 0-19) \\
178(65 ; 38-272) \\
38.2(6.9 ; 27-57) \\
214(64 ; 81-332)\end{array}$ & $\begin{array}{l}7.9(4.4 ; 0-18) \\
178(52 ; 43-277) \\
38.5(6.3 ; 24-55) \\
212(65 ; 71-370)\end{array}$ \\
\hline
\end{tabular}

Abbreviations: RDQ, Roland Disability Questionnaire; PFS, Patient-specific Functional Scale; TSK, Tampa Scale for Kinesiophobia; NMT, isometric Net Muscular Torque of the lumbar extensors (mean of 3 positions); Nm, Newtonmeter.

All therapeutic activities during treatment sessions in the regular PT program had to be written down by the physiotherapists, in order to obtain information about the content of the program. Although not every provider fully complied with this registration duty (approximately one-fifth of the treatment forms of participants in the regular PT program were not sent back), locations reported overall similar proportions of regular PT activities: approximately $65 \%$ of the activities recorded were exercise therapy (e.g., trunk and leg strengthening exercises, core stability exercises, stretching exercises, specific McKenzie exercises), 25\% aerobic activities (e.g., walking on treadmill, cycling), 10\% instruction and advice (e.g., safe 
lifting instruction, advice on proper techniques for crunches), and less than $1 \%$ were passive modalities like massage and kinesio-taping.

Despite the fact that we requested physiotherapists not to use specific devices for the lumbar extensors in the regular PT program, 2 of 6 centers reported an incidental use of standard low back machines during individual training sessions. It was not possible to quantify this any further.

Two cases of cointerventions were reported in the lumbar extensor strength training group, both providing additional manual therapy to the subject during the treatment phase. Data from these subjects were excluded from the analyses. Moreover, 1 adverse event was reported in the lumbar extensor strength training group, which concerned an acute lumbago during one of the first training sessions; the subject involved decided to discontinue the program. No adverse events were reported in the regular PT group.

Plots of the individual profiles for each outcome variable in time indicate that considerable noise is present in the data: we see a large variability in baseline values and profiles differed substantially in their form (not presented here). For example: some individuals exhibited a RMDQ profile that looked like a slanted L; others had an almost linear line with a positive slope, and others with a negative slope, etcetera. For each outcome variable, the plots shown in Figure 2 present averages for the 5 measurement occasions for lumbar extensor strength training and regular PT separately. It is evident that, on average, the treatment groups do not differ with respect to their average baseline value or the trend in time. For RMDQ and PSFS we see a slanted L-shaped pattern, with a changing slope once the follow-up phase begins. The pattern for GPE is essentially flat.

For both group, the observed mean changes over time of the 3 outcome are presented in Table 2. The results of the back strength measurements are also presented in this table, indicating that back strength progression was comparable in both groups. The frequency distribution over time of the GPE items is presented in Table 3. 


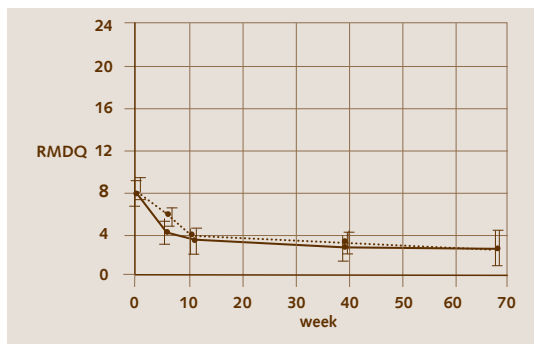

Lumbar Extensor Strength

Regular Physiotherapy
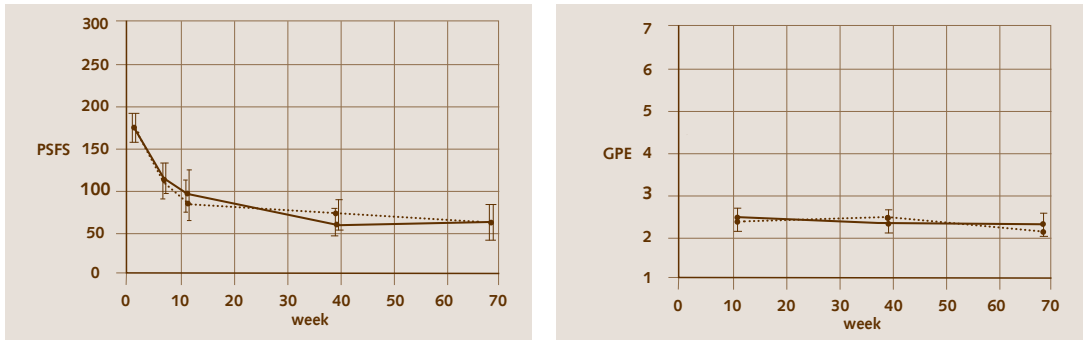

Figure 2. Means (with point-wisely constructed $95 \%$ confidence intervals) of the main outcomes over the course of the study. RDQ = Roland Disability Questionnaire (range 0-24; high score indicates greater disability); PFS = Patient-specific Functional Scale (range 0-300; high score indicates greater disability); GPE = General Perceived Effect (range 1-7; high score indicates worsening of complaints).

Table 2. Mean average value (SD) for the 3 outcome variables and for back strength per repeated measurement per treatment group.

\begin{tabular}{|c|c|c|c|c|c|c|c|c|c|c|}
\hline \multirow{2}{*}{ Week } & \multicolumn{5}{|c|}{ Lumbar Extensor Strength } & \multicolumn{5}{|c|}{ Regular Physiotherapy } \\
\hline & $\mathrm{N}$ & RDQ & PFS & GPE & NMT & $\mathrm{N}$ & RDQ & PFS & GPE & NMT \\
\hline 0 & 71 & $8.3(4.8)$ & $178(65)$ & - & $214(64)$ & 56 & $7.9(4.4)$ & $178(52)$ & - & $212(65)$ \\
\hline 5 & 64 & $5.8(4.8)$ & $119(70)$ & - & $237(62)$ & 46 & $4.2(4.2)$ & $116(67)$ & - & $246(74)$ \\
\hline 10 & 59 & $3.4(4.6)$ & $85(72)$ & $2.4(0.8)$ & $244(66)$ & 47 & $3.5(4.2)$ & $97(74)$ & $2.4(0.7)$ & $247(73)$ \\
\hline 36 & 57 & $3.2(4.3)$ & $74(72)$ & $2.5(1.0)$ & $264(64)$ & 37 & $2.7(3.8)$ & $64(59)$ & $2.3(0.9)$ & $254(73)$ \\
\hline 62 & 61 & $2.6(4.4)$ & $69(71)$ & $2.2(1.0)$ & $267(62)$ & 45 & $2.5(3.9)$ & $65(69)$ & $2.3(1.0)$ & $249(74)$ \\
\hline
\end{tabular}

Abbreviations: RDQ indicates Roland Disability Questionnaire; PFS, Patient-specific Functional Scale; GPE, Global Perceived Effect; NMT Isometric (Net) Muscular Torque (mean of 3 positions). 
Table 3. Frequency distribution (in \%) for GPE per repeated measurement per treatment group.

\begin{tabular}{|c|c|c|c|c|c|c|c|c|c|c|c|c|c|c|c|c|}
\hline \multirow{2}{*}{ Week } & \multicolumn{8}{|c|}{ Lumbar Extensor Strength } & \multicolumn{8}{|c|}{ Regular Physiotherapy } \\
\hline & $\mathrm{n}$ & $1^{\mathrm{a}}$ & 2 & 3 & 4 & 5 & 6 & 7 & $\mathrm{n}$ & 1 & 2 & 3 & 4 & 5 & 6 & 7 \\
\hline 10 & 59 & 7 & 61 & 20 & 10 & 2 & 0 & 0 & 46 & 4 & 61 & 24 & 11 & 0 & 0 & 0 \\
\hline 36 & 62 & 15 & 44 & 27 & 13 & 0 & 1 & 0 & 48 & 15 & 50 & 23 & 12 & 0 & 0 & 0 \\
\hline 62 & 64 & 27 & 39 & 27 & 6 & 0 & 1 & 0 & 50 & 20 & 46 & 18 & 12 & 4 & 0 & 0 \\
\hline
\end{tabular}

Abbreviations: GPE score (1=completely recovered, 2=much improved, 3=slightly improved, 4=no change, $5=$ slightly worsened, $6=$ much worsened, $7=$ vastly worsened).

Table 4 shows the most important results of the a priori models for the 3 outcome variables. In particular, we see that the slopes of the lumbar extensor strength training and regular PT groups are significantly different from 0 in the treatment phase. For RMDQ, 10 weeks of treatment are estimated to yield on average a 3.80-point (i.e., $10 \times-0.380$ ) drop for the lumbar extensor strength training group and a 3.55-point drop for the regular PT group. Analogously, for PSFS, 10 weeks of treatment are estimated to yield on average a 75.11-point and a 69.03-point drop for the lumbar extensor strength training and regular PT groups, respectively. While these values indicate a slight advantage for the lumbar extensor strength training group, the between-group differences (treatment effect) are not significant ( $p=.66$ for RMDQ and $p=.57$ for PFS).

For the follow-up phase, the estimated slopes indicate no significant change for RMDQ and GPE in both groups. During the 52 follow-up weeks, RMDQ is estimated to change on average by -.676 points (i.e., $52 *$-0.013) for both groups. The estimated between-group difference was essentially zero and we can therefore conclude that there was no significant treatment effect ( $p=.99)$. GPE is estimated to change by -.208 points (lumbar extensor strength training) and -.104 points (regular PT) during the 52 follow-up weeks, respectively, with again no significant difference between the 2 groups $(p=.66)$. For PFS, the estimated change was -14.872 for the lumbar extensor strength training group and -22.984 for the regular PT group. The change for the regular PT group was significantly different from $0(p=.02)$, but just failed to be significant for the lumbar extensor strength training group $(p=.08)$. Nevertheless, a direct test of the between-group difference indicated no significant treatment effect $(p=.58)$. 
Table 4. Output of mixed effects model for the main outcomes in the Lumbar Extensor Strength and Regular Physiotherapy program.

\begin{tabular}{|c|c|c|c|c|}
\hline Outcome & Phase & Parameter & Slope & $95 \% \mathrm{Cl}$ \\
\hline $\mathrm{RDQ}$ & treatment & $\begin{array}{l}\text { within-group average slope LET } \\
\text { within-group average slope REG } \\
\text { between-group effect (slope difference) } \\
\text { within-group average slope LET } \\
\text { within-group average slope REG } \\
\text { between-group effect (slope difference) }\end{array}$ & $\begin{array}{l}0.380^{\mathrm{a}} \\
-0.355^{\mathrm{a}} \\
-0.025 \\
-0.013 \\
-0.013 \\
0.000\end{array}$ & $\begin{array}{l}(-0.450--0.302) \\
(-0.441--0.270) \\
(-0.134-0.085) \\
(-0.030-0.004) \\
(-0.033-0.006) \\
(-0.025-0.026)\end{array}$ \\
\hline PFS & treatment & $\begin{array}{l}\text { average slope LET } \\
\text { average slope REG } \\
\text { between-group effect (slope difference) } \\
\text { average slope LET } \\
\text { average slope REG } \\
\text { between-group effect (slope difference) }\end{array}$ & $\begin{array}{c}7.511^{\mathrm{a}} \\
-6.903^{\mathrm{a}} \\
0.608 \\
-0.286 \\
-0.442^{\mathrm{a}} \\
0.136\end{array}$ & $\begin{array}{l}(-9.096--5.927) \\
(-8.619--5.187) \\
(-2.693-1.477) \\
(-0.600-0.028) \\
(-0.786--0.059) \\
(-0.344-0.616)\end{array}$ \\
\hline GPE & follow-up & $\begin{array}{l}\text { average slope LET } \\
\text { average slope REG } \\
\text { between-group effect (slope difference) }\end{array}$ & $\begin{array}{l}-0.004 \\
-0.002 \\
-0.002\end{array}$ & $\begin{array}{l}(-0.009-0.001) \\
(-0.008-0.003) \\
(-0.010-0.006)\end{array}$ \\
\hline
\end{tabular}

\footnotetext{
Abbreviations: $95 \% \mathrm{Cl}$, 95\% Confidence Interval; LET, Lumbar Extensor Strength program; REG, Regular Physiotherapy program; RDQ, Roland Disability Questionnaire (negative slope reflects improvement); PFS, Patient-specific Functional Scale (negative slope reflects improvement); GPE, Global Perceived Effect (positive slope reflects improvement).

Note: ${ }^{a} p<.05$.
}

The results for the effect modifier models indicate that baseline TSK score can account for some of the variability in baseline RMDQ and PSFS values, but not in slopes. The expected RMDQ value at baseline increased on average by 0.285 points for each point increase in TSK $(p<.001)$. The expected PFS value at baseline increased on average by 2.323 points for each point increase in TSK $(p<.01)$ Nevertheless, adding this effect modifier to the model does not change the overall conclusion that both treatment groups do not follow significantly different patterns during treatment and follow-up. The other potential effect modifier in this model, duration of complaints at baseline, appears to have no influence on baseline values or slopes of either main outcome.

Table 5 shows how many participants in both intervention groups were satisfied with the treatment allocation at baseline and whether this opinion had 
Table 5. Self-reported degree of satisfaction with treatment program; self-reported back pain evaluation in $1 / 2$ - and 1 -year follow-up period, respectively. Percentual values are presented.

\begin{tabular}{|c|c|c|c|}
\hline Pre-/post-treatment & Score Item & $\begin{array}{l}\text { LET } \\
(n=56)\end{array}$ & $\begin{array}{l}\text { REG } \\
(n=46)\end{array}$ \\
\hline $\begin{array}{l}\text { Satisfied with the allocated treatment program at } \\
\text { baseline? }\end{array}$ & $\begin{array}{l}\text { satisfied } \\
\text { neutral } \\
\text { not satisfied }\end{array}$ & $\begin{array}{r}70 \\
23 \\
7\end{array}$ & $\begin{array}{r}70 \\
28 \\
2\end{array}$ \\
\hline Opinion has changed during the program? & $\begin{array}{l}\text { yes, positively } \\
\text { no }\end{array}$ & $\begin{array}{l}37 \\
63\end{array}$ & $\begin{array}{l}37 \\
59\end{array}$ \\
\hline $\begin{array}{l}\text { Final degree of satisfaction at the end of the treat- } \\
\text { ment program? }\end{array}$ & $\begin{array}{l}\text { yes, negatively } \\
\text { (very) satisfied } \\
\text { neutral } \\
\text { not satisfied (at all) }\end{array}$ & $\begin{array}{r}0 \\
89 \\
11 \\
0\end{array}$ & $\begin{array}{r}4 \\
89 \\
9 \\
2 \\
\end{array}$ \\
\hline 1/2-year Follow-up & Score Item & $\begin{array}{l}\text { BS } \\
(n=56)\end{array}$ & $\begin{array}{l}\text { UC } \\
(n=40)\end{array}$ \\
\hline $\begin{array}{l}\text { Back pain in first half year after the end of the } \\
\text { treatment period? }\end{array}$ & $\begin{array}{l}\text { no, not at all } \\
\text { yes, incidentally } \\
\text { yes, monthly } \\
\text { yes, weekly }\end{array}$ & $\begin{array}{r}9 \\
57 \\
11 \\
23\end{array}$ & $\begin{array}{r}18 \\
63 \\
3 \\
18\end{array}$ \\
\hline $\begin{array}{l}\text { Back treatment in first half year after the end of } \\
\text { the treatment period? }\end{array}$ & $\begin{array}{l}\text { no, not at all } \\
\text { yes, fitness/back training } \\
\text { yes, physiotherapy } \\
\text { yes, specialist } \\
\text { yes, other treatment }\end{array}$ & $\begin{array}{r}30 \\
34 \\
14 \\
6 \\
16\end{array}$ & $\begin{array}{r}25 \\
58 \\
7 \\
0 \\
10\end{array}$ \\
\hline $\begin{array}{l}\text { Back pain medication in first half year after the } \\
\text { end of the treatment period? }\end{array}$ & $\begin{array}{l}\text { no } \\
\text { yes }\end{array}$ & $\begin{array}{r}95 \\
5\end{array}$ & $\begin{array}{l}83 \\
17\end{array}$ \\
\hline $\begin{array}{l}\text { Work absenteism due to back pain in first half } \\
\text { year after the end of the treatment period? }\end{array}$ & $\begin{array}{l}\text { no } \\
\text { yes }\end{array}$ & $\begin{array}{r}95 \\
5\end{array}$ & $\begin{array}{l}90 \\
10\end{array}$ \\
\hline 1-year Follow-up & Score Item & $\begin{array}{l}\text { BS } \\
(n=61)\end{array}$ & $\begin{array}{l}\text { UC } \\
(n=46)\end{array}$ \\
\hline $\begin{array}{l}\text { Back pain in second half year after the end of the } \\
\text { treatment period? }\end{array}$ & $\begin{array}{l}\text { no, not at all } \\
\text { yes, incidentally } \\
\text { yes, monthly } \\
\text { yes, weekly }\end{array}$ & $\begin{array}{r}25 \\
55 \\
2 \\
18\end{array}$ & $\begin{array}{l}22 \\
50 \\
11 \\
17\end{array}$ \\
\hline $\begin{array}{l}\text { Back treatment in second half year after the end of } \\
\text { the treatment period? }\end{array}$ & $\begin{array}{l}\text { no, not at all } \\
\text { yes, fitness/back training } \\
\text { yes, physiotherapy } \\
\text { yes, specialist } \\
\text { yes, other treatment }\end{array}$ & $\begin{array}{r}42 \\
34 \\
5 \\
1 \\
18\end{array}$ & $\begin{array}{r}35 \\
30 \\
15 \\
0 \\
19\end{array}$ \\
\hline $\begin{array}{l}\text { Back pain medication in second half year after the } \\
\text { end of the treatment period? }\end{array}$ & $\begin{array}{l}\text { no } \\
\text { yes }\end{array}$ & $\begin{array}{r}95 \\
5\end{array}$ & $\begin{array}{l}87 \\
13\end{array}$ \\
\hline $\begin{array}{l}\text { Work absenteism due to back pain in first half } \\
\text { year after the end of the treatment period? }\end{array}$ & $\begin{array}{l}\text { no } \\
\text { yes }\end{array}$ & $\begin{array}{r}95 \\
5\end{array}$ & $\begin{array}{r}91 \\
9\end{array}$ \\
\hline
\end{tabular}

Abbreviations: LET, Lumbar Extensor Strength; REG, Regular Physiotherapy. 
changed during the treatment phase. Moreover, the degree of satisfaction with the intervention at the end of the treatment period is presented. Finally, scores on questions about back pain episodes, back treatment, medication, and work absenteeism during the 6-month and 12-month follow-up period are presented. No clear differences between the 2 groups were seen in these items.

\section{Discussion}

In this study, two potentially effective treatment programs for LBP were compared. To our knowledge, this is the first controlled trial comparing isolated lumbar extensor training of such limited dimensions (about twenty 10-minute to 15-minute sessions) to regular PT in a predominantly male population with moderate subacute to chronic nonspecific LBP. In both intervention arms, main outcomes showed a favorable development over time: short-term effects (after 10 weeks of treatment) remained stable or even slightly improved throughout the 12month follow-up. However, no significant differences were found between the 2 intervention programs, both in the short-term and long-term. Specific back strengthening using a lumbar extensor isolation device does not seem to offer incremental benefits when compared to regular PT that mainly consists of general exercise therapy.

How can these results be interpreted? The results show that the observed mean short-term and long-term changes in the main outcome variables RMDQ and PSFS are, in both treatment groups, 3 to 5 times larger than expected because of background noise alone..$^{55}$ Fifty-nine to $68 \%$ of the participants rated their complaints on the GPE as completely recovered or much improved over time, which are indicated in the literature as qualifications for clinically important improvements. ${ }^{40}$ However, these changes cannot be automatically considered treatment effects but may also reflect natural history of recovery. We compared the outcomes of two active treatments without the inclusion of a nontreatment or placebo group. It was not considered ethically or practically feasible to include a nontreatment arm because the study relied on voluntary participation of subjects who visited the GPs office hour for their back pain. This precludes an adequate insight in the natural course of LBP over time in both groups. 
Definitive inferences about the effectiveness of either one of the treatments are therefore hard to make.

\section{Study limitations}

Several methodological aspects that may have influenced the outcomes can be considered, such as trial power, intervention contrast, and loss to follow-up. First, our strict inclusion and exclusion criteria (especially availability in duty time and LBP $>4 w k$ ), as well as a slow recruitment rate, resulted in a lower sample size than expected. This inevitably reduces the power of this study, and, consequently, the robustness of our findings.

Second, the intervention contrast between the lumbar extensor strength training program and regular PT program may not have been large enough to detect clinically relevant changes with the chosen outcome measures. The results show that strength progression in the lumbar extensor strength training group was comparable to the regular PT group, indicating that both interventions provided an equally strong strength training stimulus. Specific (isolated) lumbar extensor strengthening exercises were excluded from our regular PT program, but some general back strengthening exercises were allowed; the regular PT treatment would otherwise not have been a realistic representation of usual back pain management in the Dutch army. Moreover, 2 locations reported that they had used the low back training device incidentally in the regular PT program, despite our request not to do so. This may have influenced the results of the regular PT program in some way.

Third, the treatment frequency differed somewhat between both groups: 4 more treatment sessions, on average, were planned in the lumbar extensor strength training group compared with the regular PT group. The average time of a treatment session was, on the other hand, $50 \%$ shorter in the lumbar extensor strength training group. Fourth, the loss to follow-up in this trial may have influenced the results. With $8 \%$ dropouts (lumbar extensor strength training, regular PT) during the treatment period and 13\% (lumbar extensor strength training) and 18\% (regular PT) overall dropouts, respectively, we had a followup rate comparable or favorable to what has been reported in other studies on 
exercise therapy in outpatient settings. ${ }^{18,21,56-60}$ The greater part of the loss to follow-up was a result of factors unrelated to the study, such as military mission or leaving military duty. Dropouts were evenly distributed over both treatment groups, which supports the stability of our findings. The problem of relatively high numbers of missing values in our database was tackled by applying random coefficient analysis, a statistical technique that includes both complete and incomplete longitudinal data.

Next, one cannot disregard that the use of a new low back training device in the lumbar extensor strength training program as opposed to general exercises in the regular PT program had an added psychological impact on outcomes (novelty-effect). As in most exercise therapy interventions, neither participants nor providers could be blinded for the type of intervention used in this study. Although it was not possible to blind participants from expectation bias about the efficacy of the allocated treatment, we took great care in maximizing the treatment credibility of both programs when explaining the intervention to each participant. The degree of satisfaction with the allocated treatment throughout the intervention period appeared to be rather similar across the treatment groups, indicating that neither of the 2 treatment programs was seen more or less credible than the other.

Finally, to evaluate validly the lumbar extensor strength training concept in its future setting we have chosen regular visitors of our military health centers as our target population. This study population of predominantly male, young, and physically active subjects might have influenced the outcomes. Because both interventions mainly focused on exercising and strengthening the lumbar region, they might have been more effective and efficient in groups of patients (older, unfit, female) with a higher strength development potential.

The results of this study corroborate earlier studies ${ }^{19-21}$ and reviews ${ }^{13-18}$ on the same topic, indicating that many therapeutic exercise interventions exist, but none seem to be clearly superior to others. In a recent review, Slade et al conclude that the optimal type of exercise for chronic LBP is still unknown because the effects of specific exercises have not been systematically assessed..$^{14}$ Exercise 
interventions that make use of a singular concept need to be studied. In our trial we compared a unimodal back strengthening program using a test-and training device with a treatment program that included a wide range of therapeutic exercises. Our back strengthening program included elements that have recently been identified as important intervention characteristics to decrease back pain and improve function, such as exercises that are individually designed, that contain muscle strengthening, and that are supervised. ${ }^{61}$

The results of this study can be extrapolated to other working populations with a predominance of male employees (e.g., construction workers, police squads, fire brigades). We do not expect the long recruitment process to have significantly influenced the generalizability of this study because the (military) population at risk can be considered relatively stable and homogeneous. In our opinion, our inclusion and exclusion criteria were well-defined, leaving no room for GPs of different health centers to refer certain subgroups of patients more often to the study. Subjects in this study were selected from a patient group normally considered suitable for progressive resistance training inasmuch all patients with clinical contraindications (e.g., affected nerve root) were excluded.

\section{Conclusions}

Our study indicates that neither specific isolated lumbar extensor training with a training device nor regular PT that mainly consist of general exercise therapy is more effective than the other in a predominantly male population with moderate subacute to chronic nonspecific LBP.

Acknowledgments: We thank Peter de Putter and Wim Veenman for their efforts in the development and maintenance of the ALBATROS software program, as well as in the management of all data. We thank Colonel René Roelofs, MD, former Surgeon General RNLA, and Colonel Kees IJzerman, MD, former Director Occupational Health \& Safety Service RNLA, for authorizing this multicenter study within the military setting. 


\section{References}

1. Andersson GB. Epidemiological features of chronic low-back pain. Lancet 1999; 354:581-5.

2. Hutubessy RC, van Tulder MW, Vondeling $H$, Bouter $L M$. Indirect costs of back pain in the Netherlands: A comparison of the human capital method with the friction cost method. Pain 1999;80:201-7.

3. Morken $\mathrm{T}$, Riise $\mathrm{T}$, Moen $\mathrm{B}$, Bergum $\mathrm{O}$, Hange $\mathrm{SH}$, Holien $\mathrm{S}$, Langedrag A, Olsen $\mathrm{HO}$, Pedersen S, Saue IL, Seljebo GM, Thoppil V. Frequent musculoskeletal symptoms and reduced health-related quality of life among industrial workers. Occup Med (Lond) 2002;52:91-8.

4. Morken T, Riise T, Moen B, Bergum O, Bua L, Hange SH, Holien S, Langedrag A, Olsen HO, Pedersen S, Saue IL, Seljebo GM, Thoppil V. Low back pain and widespread pain predict sickness absence among industrial workers. BMC Musculoskelet Disord 2003;4:21.

5. Berkowitz SM, Feuerstein M, Lopez MS, Peck CA Jr. Occupational back disability in U.S. Army personnel. Mil Med 1999;164:412-8.

6. Lahiri S, Markkanen P, Levenstein C. The cost effectiveness of occupational health interventions: Preventing occupational back pain. Am J Ind Med 2005;48:515-29.

7. Hashemi L, Webster BS, Clancy EA, Volinn E. Length of disability and cost of workers' compensation low back pain claims. J Occup Environ Med 1997;39:937-45.

8. Alexanderson KA, Borg KE, Hensing GK. Sickness absence with low back, shoulder, or neck diagnoses: An 11-year follow-up regarding gender differences in sickness absence and disability pension. Work 2005;25:115-24.

9. Feuerstein M, Berkowitz SM, Peck CA. Musculoskeletal-related disability in US Army personnel: Prevalence, gender, and military occupational specialties. J Occup Environ Med 1997;39:68-79

10. Van Tulder MW, Koes B, Bombardier C. Low back pain. Best Pract Res Clin Rheumatol 2002;16:761-75.

11. Marras WS. Occupational low back disorder causation and control. Ergonomics 2000;43:880-902.

12. Burdorf A, Sorock G. Positive and negative evidence of risk factors for back disorders. Scand J Work Environ Health 1997;23:243-56.

13. Van Tulder MW, Koes B, Malmivaara A. Outcome of non-invasive treatment modalities on back pain: An evidence-based review. Eur Spine J 2006;15 Suppl 1:S64-81.

14. Slade SC, Keating JL. Trunk-strengthening exercises for chronic low back pain: A systematic review. J Manipulative Physiol Ther 2006;29:163-73.

15. Hayden JA, van Tulder MW, Malmivaara AV, Koes BW. Meta-analysis: exercise therapy for nonspecific low back pain. Ann Int Med 2005;142:776-85.

16. Taylor NF, Dodd KJ, Damiano DL. Progressive resistance exercise in physical therapy: A summary of systematic reviews. Phys Ther 2005;85:1208-23.

17. Liddle SD, Baxter GD, Gracey JH. Exercise and chronic low back pain: What works? Pain 2004;107:176-90.

18. Rainville J, Hartigan C, Martinez E, Limke J, Jouve C, Finno M. Exercise as a treatment for chronic low back pain. Spine J 2004;4:106-15.

19. Ferreira ML, Ferreira PH, Latimer J, Herbert RD, Hodges PW, Jennings MD, Maher CG, 
Refshage KM. Comparison of general exercise, motor control exercise and spinal manipulative therapy for chronic low back pain: A randomized trial. Pain 2007;131:31-7.

20. Cairns MC, Foster NE, Wright C. Randomized controlled trial of specific spinal stabilization exercises and conventional physiotherapy for recurrent low back pain. Spine 2006;31:E670-81.

21. Mannion AF, Muntener M, Taimela S, Dvorak J. A randomized clinical trial of three active therapies for chronic low back pain. Spine 1999;24:2435-48.

22. Bouter LM, van Tulder MW, Koes BW. Methodologic issues in low back pain research in primary care. Spine 1998;23:2014-20.

23. Leboeuf-Yde C, Lauritsen JM, Lauritzen T. Why has the search for causes of low back pain largely been nonconclusive? Spine 1997;22:877-81.

24. Helmhout PH, Harts CC, Staal JB, de Bie RA. Rationale and design of a multicenter randomized controlled trial on a 'minimal intervention' in Dutch army personnel with nonspecific low back pain [ISRCTN19334317]. BMC Musculoskelet Disord 2004;5:40.

25. Helmhout PH, Harts CC, Staal JB, Candel MJ, de Bie RA. Comparison of a high-intensity and a low-intensity lumbar extensor training program as minimal intervention treatment in low back pain: A randomized trial. Eur Spine J 2004;13:537-47.

26. Leggett S, Mooney V, Matheson LN, Nelson B, Dreisinger T, Van Zytveld J, Vie L. Restorative exercise for clinical low back pain: A prospective two-center study with 1-year follow-up. Spine 1999;24:889-98.

27. Nelson BW, O'Reilly E, Miller M, Hogan M, Wegner JA, Kelly C. The clinical effects of intensive, specific exercise on chronic low back pain: A controlled study of 895 consecutive patients with 1-year follow up. Orthopedics 1995;18:971-81.

28. Risch SV, Norvell NK, Pollock ML, Risch ED, Langer H, Fulton M, Graves JE, Leggett SH. Lumbar strengthening in chronic low back pain patients: Physiologic and psychological benefits. Spine 1993;18:232-8.

29. Miltner O, Wirtz DC, Siebert CH. [Strengthening lumbar extensors--therapy of chronic back pain-an overview and meta-analysis]. Z Orthop Ihre Grenzgeb 2001;139:287-93.

30. Manniche C, Hesselsoe G, Bentzen L, Christensen I, Betzen L, Lundberg E. Clinical trial of intensive muscle training for chronic low back pain. Lancet 1988;2:1473-6.

31. Manniche C, Lundberg E, Christensen I, Bentzen L, Hesselsoe G. Intensive dynamic back exercises for chronic low back pain: a clinical trial. Pain 1991;47:53-63.

32. Harts CC, Helmhout PH, de Bie RA, Staal JB. Comparison of high-intensity lumbar extensor training for chronic low back pain with low-intensity training and a waitinglist control group: a randomised clinical trial. Austr J Physiother. Submitted.

33. Fleck SJ, Kraemer WJ. Designing resistance training programs. 2nd ed. Champaign (IL): Human Kinetics; 1997.

34. Pollock ML, Leggett SH, Graves JE, Jones A, Fulton M, Cirulli J. Effect of resistance training on lumbar extension strength. Am J Sports Med 1989;17:624-9.

35. Carpenter DM, Graves JE, Pollock ML, Leggett SH, Foster D, Holmes B, Fulton MN. Effect of 12 and 20 weeks of resistance training on lumbar exension torque production. Phys Ther 1991;71:580-8.

36. Tucci JT, Carpenter DM, Pollock ML, Graves JE, Leggett SH. Effect of reduced frequency of training and detraining on lumbar extension strength. Spine 1992;17:1497-501.

37. American College of Sports Medicine. ACSM's guidelines for exercise testing and 
prescription. Philadelphia, PA: Lippincott Williams \& Wilkins, 2000.

38. Gommans I, Koes B, Van Tulder M. Validiteit en responsiviteit Nederlandse Roland Disability Questionnaire: Vragenlijst naar functionele status bij patiënten met lage rugpijn. Ned Tijdschr Fysiother 1997;2:28-33.

39. Roland M, Morris R. A study of the natural history of back pain. Part I: development of a reliable and sensitive measure of disability in low-back pain. Spine 1983;8:141-4.

40. Beurskens AJ, de Vet HC, Koke AJ, Lindeman E, van der Heijden GJ, Regtop W, Knipschild PG. A patient-specific approach for measuring functional status in low back pain. J Manipulative Physiol Ther 1999;22:144-8.

41. Beurskens AJ, de Vet HC, Koke AJ. Responsiveness of functional status in low back pain: A comparison of different instruments. Pain 1996;65:71-6.

42. Kori SH, Miller RP, Todd DD. Kinesiophobia: A new view of chronic pain behavior. Pain Management 1990;Jan/Feb:35-43

43. Goubert L, Crombez G, Vlaeyen J, van Damme S, van den Broeck A, van Houdenhove B. De Tampa schaal voor kinesiofobie: Psychometrische karakteristieken en normering. Gedrag Gezond 2000;28:54-62.

44. Vlaeyen JW, Kole-Snijders AM, Boeren RG, van Eek H. Fear of movement/(re)injury in chronic low back pain and its relation to behavioral performance. Pain 1995;62:363-72.

45. Bouter LM, van Dongen MC. Epidemiologisch onderzoek: Opzet en interpretatie. 3th ed. Houten/Diegem: Bohn Stafleu Van Loghum; 1995.

46. Rosner BA. Fundamentals of biostatistics. 4th ed. Belmont: Duxbury Press; 1995.

47. Resnik L, Dobrzykowski E. Guide to outcomes measurement for patients with low back pain syndromes. J Orthop Sports Phys Ther 2003;33:307-16; discussion 17-8.

48. Pinheiro JC, Bates DM, DebRoy S, Sarkar D. Linear and nonlinear mixed effects models. Version 3.1-80; 2007. Available from: URL: http://www.R-project.org.

49. R Developement Core Team. R: a language and environment for statistical computing. Vienna, Austria: R Foudation for Statistical Computing; 2007. Available from: URL: http://www.R-project.org.

50. Babyak MA. What you see may not be what you get: a brief, nontechnical introduction to overfitting in regression-type models. Psychosom Med 2004;66:411-21.

51. Bekkering GE, Hendriks HJ, van Tulder MW, Knol DL, Simmonds MJ, Oostendorp RA, Bouter LM. Prognostic factors for low back pain in patients referred for physiotherapy: comparing outcomes and varying modeling techniques. Spine 2005;30:1881-6.

52. Fritz JM, George SZ. Identifying psychosocial variables in patients with acute workrelated low back pain: The importance of fear-avoidance beliefs. Phys Ther 2002;82:973-83

53. Picavet HS, Vlaeyen JW, Schouten JS. Pain catastrophizing and kinesiophobia: Predictors of chronic low back pain. Am J Epidemiol 2002;156:1028-34.

54. Pincus T, Burton AK, Vogel S, Field AP. A systematic review of psychological factors as predictors of chronicity/disability in prospective cohorts of low back pain. Spine 2002;27:E109-20.

55. De Vet HC, Bouter LM, Bezemer PD. Reproducibility and responsiveness of evaluative outcome measures: Theoretical considerations illustrated by an empirical example. Int J Technol Assess Health Care 2001;17:479-87.

56. Petersen T, Kryger P, Ekdahl C, Olsen S, Jacobsen S. The effect of McKenzie therapy 
as compared with that of intensive strengthening training for the treatment of patients with subacute or chronic low back pain: A randomized controlled trial. Spine 2002;27:1702-9.

57. Kuukkanen T, Malkia E. Effects of a three-month therapeutic exercise programme on flexibility in subjects with low back pain. Physiother Res Int 2000;5:46-61.

58. Kankaanpaa M, Taimela S, Airaksinen $O$, Hanninen O. The efficacy of active rehabilitation in chronic low back pain: Effect on pain intenty, self-experienced disability, and lumbar fatigability. Spine 1999;24:1034-42.

59. Bendix AF, Bendix T, Labriola M, Haestrup C, Ebbehoj N. Functional restoration for chronic low back pain: 2-year follow-up of 2 randomized clinical trials. Spine 1998;23:717-25.

60. Hansen FR, Bendix T, Skov P, Jensen CV, Kristensen JH, Krohn L, Schioeler H. Intensive, dynamic back-muscle exercises, conventional physiotherapy, or placebo-control treatment of low-back pain: A randomized, observer-blind trial. Spine 1993;18:98-108.

61. Hayden JA, van Tulder MW, Tomlinson G. Systematic review: strategies for using exercise therapy to improve outcomes in chronic low back pain. Ann Intern Med 2005;142:776-85. 



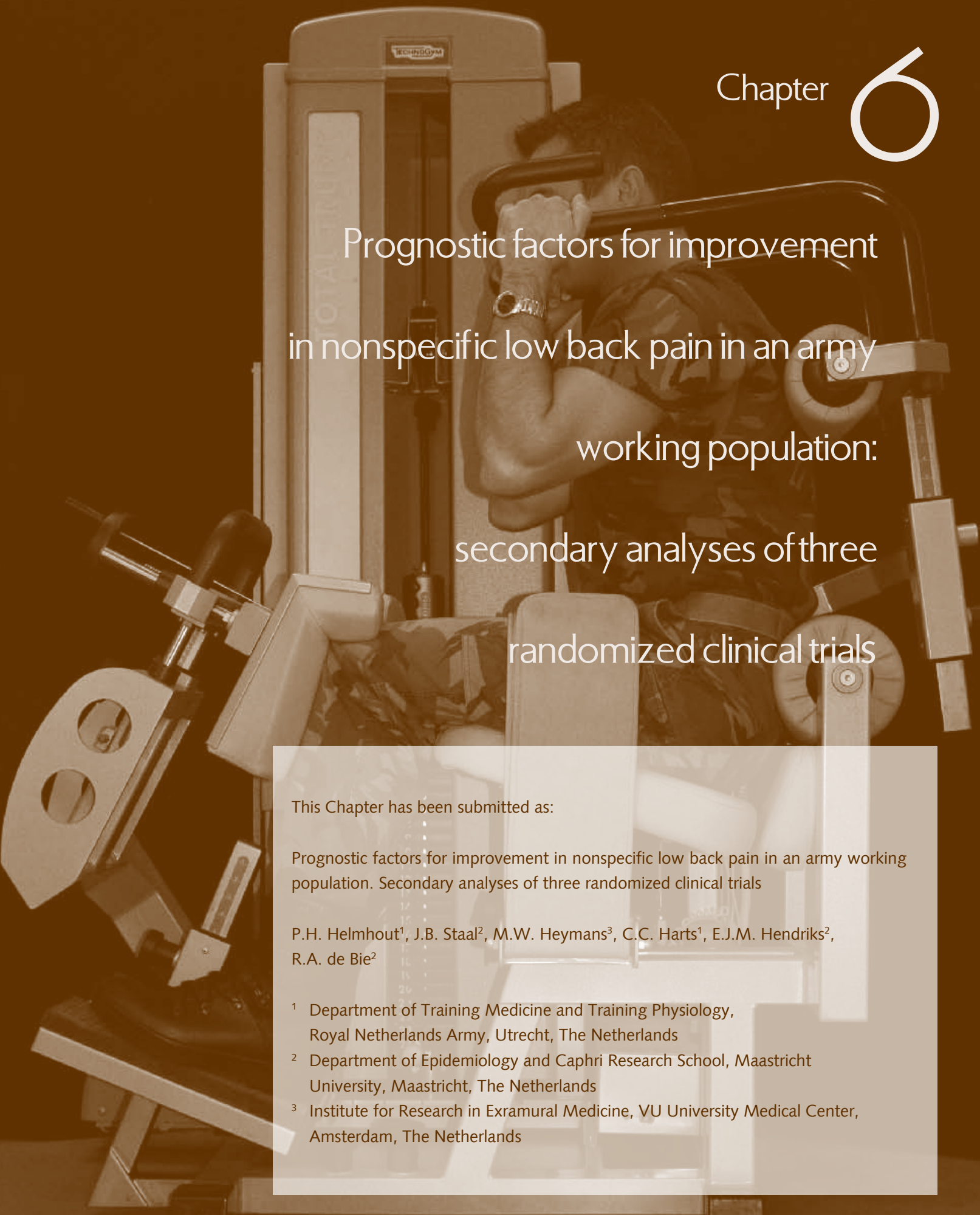




\section{Abstract}

Objectives: The objective of this study was to to report on secondary analyses of a merged trial dataset aimed at exploring the potential importance of patient factors associated with clinically relevant improvements in nonacute nonspecific low back pain (LBP).

Methods: From 273 predominantly male army workers (mean age, $39 \pm 10.5$ years; range 20-56 years, 4 women) with LBP who were recruited in three randomized clinical trials, baseline individual patient factors, pain-related factors, work-related psychosocial factors, and psychological factors were evaluated as potential prognostic variables in a short-term (post-treatment) and a long-term logistic regression model (6 months after treatment).

Results: We found one dominant prognostic factor for improvement directly after treatment as well as 6 months later: baseline functional disability, expressed in Roland-Morris Disability Questionnaire scores. Baseline fear of movement, expressed in Tampa Scale for Kinesiophobia scores, had also significant prognostic value for long-term improvement. Less strongly associated with the outcome, but also included in our final models, were supervisor social support and duration of complaints (short-term model), and co-worker social support and pain radiation (long-term model).

Discussion: Information about initial levels of functional disability and fear-avoidance behaviour can be of value in the treatment of patient populations with characteristics comparable to the current army study population (e.g., predominantly male, physically active, working, moderate but chronic back problems). Individuals at risk for poor long-term LBP recovery, i.e., individuals with high initial level of disability and prominent fear-avoidance behaviour, can be distinguished that may need additional cognitive-behavioural treatment. 


\section{Introduction}

Low back pain (LBP) is seen as a largely self-limiting health problem, with rapid improvement usually occuring within several weeks. ${ }^{42}$ However, once the pain is showing itself as a recurrent and chronic phenomenon, it is mostly associated with long-term disability and, consequently, a significant socioeconomic burden: some $80 \%$ of the health care and social costs are attributed to the $10 \%$ of cases with chronic pain and disability. ${ }^{39}$

Accurately identifying individuals with a good or unfavourable prognosis among patients presenting with LBP is an important goal in current back pain research. Being able to predict prognosis of LBP patients based on pre-treatment assessment of patient characteristics may lead to more realistic expectations of recovery, as well as to more effective and efficient use of treatment modalities in the prevention of chronicity. ${ }^{32}$ The identification of factors that can or cannot be modified in patients who are at risk for developing longstanding LBP, may facilitate the selection of patients who will most likely benefit from targeted treatment. For example, if fear of movement appears to hinder a favourable prognosis in a certain subgroup of LBP patients, future exercises designed to mobilize or strengthen vulnerable lumbar body parts may become more effective in this target population when assisted by educational information and behavioural strategies regarding fear-related pitfalls. ${ }^{11}$

Despite the fact that a considerable amount of research has been accumulated on a wide range of prognostic factors for LBP, inconsistencies among study results have limited the strength of conclusions. These inconsistencies have partly been attributed to methodological weaknesses of the studies involved, i.e., recruitment of heterogeneous cohorts in different settings and on different timepoints; lack of an overarching conceptual framework; different use of measurements; model building with more variables than justified for the given number of observations; and/or incorrect use of statistical regression methods. 1,2,8,32,43,45 Performing studies of sufficient statistical power on relatively homogeneous back-injured populations, preferably with a core set of measurements that are supported by the literature, may be of use for future reviews and meta-analyses 
that aim to identify those factors most strongly related to the onset and recurrence of LPB.

In recent years, we have conducted three randomized clinical trials to evaluate the effectiveness of different exercise modalities in an army working population with nonacute nonspecific LBP. ${ }^{24-27}$ We provided isolated lumbar extensor strengthening versus mobilization versus general exercise therapy as our treatment interventions. Participants were 273 predominantly male soldiers from the Royal Netherlands Army with 4 weeks or more of low back complaints, who were referred to physiotherapy by the general practitioner of the military health center. Consistent with prior evidence, 5,13,36 neither of the exercise modalities seemed to offer incremental improvements over the other, up to one year posttreatment.

For several reasons, the data from these trials could be of value in secondary analyses aimed at identifying prognostic factors for clinically important LBP improvements. First, our study population can be considered relatively homogeneous. Inclusion and exclusion criteria were well-defined and comparable among trials. Subjects were selected from a patient group normally considered suitable for progressive resistance training, i.e., all patients with clinical contraindications (e.g., affected nerve root) were excluded. Participants were all professional military employees and predominantly male (in total only 4 women were included), reflecting the vast male majority in our organization. Most participants were military recruits (younger population), military instructors (older population) or military staff personnel. All participants were working at the time of their inclusion, with, in many cases, similar physical and mental job demands. Overall, the large majority of our study population perceived their work as non-physical, despite the fact that working in uneasy or fixed positions and frequent lifting/carrying of heavy loads were reported in many cases. More than half of the participants were physically active in daily life despite their mostly moderate back complaints. This may be a reflection of the more-than-average physical attitude of military personnel in general; physical fitness is a critical aspect of military readiness and an inherent part of military service. Initial scores on a self-perceived health questionnaire (SF-36) that was used in two of our three trials indicated that our 
study population tend to attribute unspecific physical symptoms towards somatic disease more than towards mental issues. ${ }^{24,25}$ Furthermore, the study groups of our three trials were similar at baseline on most potential prognostic factors. The proportions of patients showing a favourable long-term outcome were also comparable among the three trials (71\%, $73 \%$, and 69\%, respectively). Finally, the three trials had several common measures that could be used for pooled analyses. These measures have recently been recognized and included in a core set of factors for prospective cohorts in LBP. ${ }^{45}$

The purpose of this paper is to report on secondary analyses of a merged trial dataset aimed at exploring the prognostic value of individual patient factors, pain-related factors, work-related psychosocial factors, and psychological factors, in nonacute nonspecific LBP. By identifying these prognostic factors, LBP management could be improved by adjusting current therapy concepts or by targeting therapies at those likely to gain the greatest benefit.

\section{Methods}

\section{Study design}

We used a prospective cohort design by merging data from three randomized trials on the effectiveness of exercise therapy in individuals with nonspecific LBP. Patients enrolled in the trials were randomized into an intervention group that received either an 8- to 12-week, high-intensive or low-intensive isolated lumbar extensor training program using specific training devices, or usual care that mainly consisted of general exercise therapy. Average numbers of treatment sessions varied from 8 to 14, depending on the program. Mean intervention times per treatment session varied from 10 to 15 minutes (lumbar extensor training), and from 25 to 30 minutes (regular physiotherapy). An extensive description of the design and results of these trials have been published in recent years. ${ }^{24-27}$

\section{Subjects}

The source population consisted of professional military employees of the Royal Netherlands Army ( $n=$ approx. 23,000). In total, 273 predominantly male participants (mean age, $39 \pm 10.5$ years; range 20-56 years, 4 women) were 
recruited during regular GP consulting hours as well as through advertisement in military media. None of the participants were performing combat related activities during their follow-up in the trials. Inclusion criteria were: at least 4 weeks of continuous LBP or recurrent (at least 3 times a week) episodes of LBP; pain localized between posterior iliac crests and angulus inferior scapulae; availability during duty time; and willingness to abandon other treatment interventions for the lower back during the intervention period. Exclusion criteria were: received spinal surgery in the last 2 years; specific treatment for LBP in the last 4 weeks (e.g., physiotherapy; manual therapy); severe LBP which hindered performing maximal isometric strength efforts; specific LBP, defined as herniated disk, ankylosing spondylitis, spondylolisthesis, or relevant neurological diseases. All three trials used comparable methods to collect demographic and clinical information prior to randomization.

\section{Prognostic factors}

At baseline, directly after treatment and 6 months after treatment, respectively, patients completed a compound questionnaire containing, amongst others, the following items:

- functional disability, measured with the 24-item Roland-Morris Disability Questionnaire (RMDQ);49

- duration of LBP complaints at baseline, categorized as: 4-6 weeks; 6-12 weeks; 3-6 months; 6-12 months; 1 year or more. For analyses purposes, we dichotomized this variable, using a cut-off point of one year to form balanced groups of patients with a shorter and longer duration of complaints, respectively;

- pain radiation or tingling in the legs at baseline (yes/no);

- fear of movement, measured with the validated Tampa Scale for Kinesiophobia (TSK); 37,48

- psychological distress, using the 12-item General Health Questionnaire $(\mathrm{GHQ}), 20$ and only measured in the $3^{\text {rd }}$ trial $(n=127)$;

- subscales 'Supervisor Social Support' and 'Co-worker Social Support' of the Job Content Questionnaire (JCQ),31 only measured in the $3^{\text {rd }}$ trial $(n=127)$;

- degree of physical activity, measured in the first two trials with the criterion 
'physically active for at least 30 minutes per day', ${ }^{41}$ and, in the $3^{\text {rd }}$ trial, with the validated Short Questionnaire to Assess Health Enhancing Physical Activity (SQUASH). ${ }^{64}$

Baseline values of these 8 variables (RMDQ; LBP duration; pain radiation; TSK; GHQ; JCQ subscales; physical activity) were included in the analyses as potential prognostic factors. The choice to include these factors in our analyses was based on the fact that: (a) they were considered core prognostic factors in at least two out of three recent reviews on prospective cohorts in persistent LBP disability, 8,45,52 or (b) they were considered relevant for the population under study, based on earlier experience (e.g., degree of physical activity).

\section{Outcome}

Patient improvement was selected as the dependent variable in our prognostic model. This variable was composed of changes in RMDQ scores and self-assessed changes in back complaints, post-treatment and after 6 months of followup, respectively. Self-assessed change in back complaints since the start of the treatment was scored on a percentual scale (0 to 100\% improvement) in the first two trials, ${ }^{24,25}$ or the 7-item Global Perceived Effect (GPE: 1=completely recovered, $2=$ much improved, $3=$ slightly improved, $4=$ no change, $5=$ slightly worsened, $6=$ much worsened, $7=$ vastly worsened) in the $3^{\text {rd }}$ trial, ${ }^{3}$ respectively. The outcome variable was dichotomized into 'improved' and 'non-improved'. We defined 'improved' defined as subjects who met both the following criteria:

- $30 \%$ or more of improvement on the RMDQ;

- a score 'completely recovered' or 'much improved' on the GPE, or $20 \%$ or more of self-assessed improvement on the percentual scale.

These criteria were partly derived from recommendations by Jordan et al on clinically important differences in LBP, based on the RMDQ..$^{30}$

\section{Statistical Analyses}

\section{Model building}

Prognostic variables and outcome variables with incomplete baseline and followup data were completed by using the Multiple Imputation by Chained Equations 
(MICE) procedure. ${ }^{58}$ In a multiple imputation procedure, each missing value is replaced by a set of multiple different values. These values are estimated by using regression models and all available data. We generated 5 multiply imputed datasets, according to Schafer. ${ }^{51}$

The relationship between the outcome directly after treatment and 6 months after treatment, respectively, and each of the potential prognostic factors was individually calculated, using univariate logistic regression analyses. The allocated trial intervention was included in all analyses. Univariate odds ratios (OR) were calculated to reflect the strength of each relationship, together with the corresponding $95 \%$ confidence interval $(95 \% \mathrm{Cl})$.

To determine which combination of factors were related to the outcome, we included all 8 potential prognostic factors in a multivariable logistic regression model. This takes into account the 'rule of thumb' in logistic regression that the number of the less common of the two possible outcomes (in our case: 'nonimproved' with, on average, 96 cases post-treatment and 89 cases at follow-up) divided by the maximum number of prognostic factors in the model should be at least 10.42 Backward regression analysis was applied to build each model, using a variable selection method that has recently been recommended by Wood and Royston. ${ }^{65}$ With this method, backward regression is performed taking into account all imputed datasets. The initial regression model including all potential prognostic factors is fitted on each imputed dataset. Regression coefficients and related standard errors and $p$-values are then estimated over all multiply imputed datasets by using Rubin's Rules. ${ }^{50}$

Then, like with 'normal' backward regression, the variable with the highest $p$ value is first omitted from the model. This smaller model is again fitted on each imputed dataset and, again, the variable with the largest $p$-value is omitted. This procedure is repeated until all variables with a $P$ value of $<.50$ are retained in the model, following recommendations by Steyerberg et al. ${ }^{55} \mathrm{~A}$ liberal $\mathrm{P}$ value increases the chances of obtaining true predictors, limiting the bias in selected coefficients. 
Model performance

The goodness-of-fit of the each model was verified with the Hosmer-Lemeshow test. ${ }^{29} \mathrm{~A}$ nonsignificant $\mathrm{Chi}^{2}$-value $(\alpha=.05)$ in this test is indicative of a good model fit. In addition, we used residual regression diagnostics (Cook's Distance, Leverage, Studentized Residual, and DFBeta) in revealing the effect on the estimated models of individual observations that are not adequately described by the model or that are highly influential on the model fit. ${ }^{15}$ Moreover, we used collinearity diagnostics to check if factors were highly correlated. ${ }^{15} \mathrm{~A}$ potential nonlinear behaviour of the continuous factors with the outcome was examined by using restricted cubic spline functions and spline plots. Restricted cubic spline functions allow continuous indicators to be fitted within the regression model without assuming a linear relation. ${ }^{23}$ We did find a nonlinear relation for baseline RMDQ score and, therefore, included this variable in restricted cubic spline form in our model selection process. The spline variable was converted into a dummy variable (quartile categories with scores $<4,4-7,7-11, \geq 11$ ) in our final model to enhance clinical interpretation. We checked if the interpretation of the OR's would change if we fitted the models without the RMDQ spline function, which was not the case. All goodness-of-fit analyses were applied on the first imputed dataset, the results of which were comparable to those of the other datasets.

Two measures were used to further assess model performance: Nagelkerke's R2 and the C-index. Nagelkerke's $R^{2}\left(R_{N}^{2}\right)$ is an approximation of the explained variance $\left(R^{2}\right)$ concept for the ordinary regression model. The $C$-index, calculated as the area under the curve of the receiver operating characteristic (ROC) plot, represents the concordance between predicted probabilities and observed outcomes for all possible pairs of patients. It, therefore, indicates the discriminative ability of the logistic model. A C-index of 1.0 indicates perfect discrimination while a value of 0.5 indicates the model performs no better than chance alone. Prognostic models usually perform better in the patient sample that was used to build the model than in other new patient samples, due to optimism in regression coefficients and performance measures. To estimate the amount of optimism in the $\mathrm{C}$-index and explained variance, we used bootstrapping techniques. ${ }^{47}$ The model performance indices were calculated on each of the 5 imputed dataset and then averaged. 


\section{Software}

Imputation, backward selection, and bootstrapping were performed with $\mathrm{R} .{ }^{47}$ Diagnostic analyses were performed using SAS Version 9.1 (goodness-of-fit) and SPSS for Windows Version 15.0 (regression diagnostics, multicollinearity).

\section{Results}

The merged dataset of the three previous trials consisted of 273 subjects with nonacute nonspecific LBP. Table 1 shows the characteristics of these subjects at baseline. The percentage of missing data of the potential prognostic factors before imputing varied from 0 to $9 \%$ save those that were only measured in the $3^{\text {rd }}$ trial, i.e., psychological distress, and supervisor/co-worker social support (53\% missing overall). From the original dataset, 119 out of 225 subjects (53\%) were labelled as 'improved' directly after treatment, and 131 out of 210 subjects (62\%) at 6 months of follow-up. From the overall study population of 273 participants, only $3 \%(\mathrm{~N}=9)$ had a zero score on the RMDQ at baseline. All nine subjects scored moderate to substantial self-assessed improvements in back complaints on either of the other scales that contribute to our outcome variable.

Table 2 shows the analyses of the individual prognostic factors, indicating that baseline RMDQ score between 8 and 11 was significantly associated with improvement in LBP, both directly $(O R, 3.57)$ and 6 months after treatment $(O R$, 4.22). Baseline RMDQ scores between 4 and 7 (OR, 2.29) and scores of 11 and more (OR, 2.53), respectively, as well as baseline TSK score $(O R, 0.97)$ were significantly associated with long-term improvement.

The multivariate analyses (see Table 3) showed a final post-treatment model that included four prognostic factors, together explaining $12 \%$ of the variation in outcome: functional disability, fear of movement, supervisor social support, and duration of complaints. All other factors were eliminated due to the $\mathrm{P}<.50$ criterion for backward regression. The final long-term model consisted of the following five factors (16\% explained variance): functional disability, fear of movement, psychological distress, co-worker social support, and pain radiation. In the post-treatment model, the prognostic factor most strongly associated with 
improvement was a baseline RMDQ score between 8 and 11 (OR, 3.98). In the long-term model, baseline RMDQ scores (OR's 2.97-7.31), as well as baseline TSK score (OR, 0.91) were the strongest prognostic factors.

Both models showed fairly good discriminative power with a C-index of 0.680.70. Moreover, the global goodness-of-fit test was not rejected (see Table 3) and the regression diagnostics were within normal ranges indicating adequate model fit (not presented). Collinearity diagnostics showed that the assumption of 'no multicollinearity' was met in both models (not presented).

\section{Discussion}

The aim of this study was to assess the relative importance of individual patient factors, pain-related factors, work-related psychosocial factors, and psychological factors, in explaining self-reported clinically important improvements in LBP complaints. Overall, we found one dominant prognostic factor for improvement directly after treatment as well as 6 months later: functional disability, more specifically intermediate RMDQ score at baseline. Fear of movement had also significant prognostic value for long-term improvement. These factors were also found to be individually related to the outcome in the univariate analyses. Less strongly associated with the outcome, but also included in our final multivariate models, were supervisor social support and duration of complaints (short-term model), and co-worker social support and pain radiation (long-term model).

The consistent appearance of the two strongest prognostic factors, functional disability and fear of movement, in our final models is in line with earlier prognostic LBP studies, in the sense that they are considered as important and independent determinants of many different LBP outcomes (e.g., remitting pain; sub-acute or chronic disability; failed or delayed recovery from short-term LBP; long-term compensation status; time to return-to-work) in various study settings (e.g., primary care; specialist back clinic; occupational health care; mailing survey). Concordant with other studies on $L B P, 6,7,12,16,40$ functional disability (baseline RMDQ) was highly predictable in our models. Table 3 illustrates that midrange scores on the RMDQ (score 8-11) and, to a lesser extent, high range 


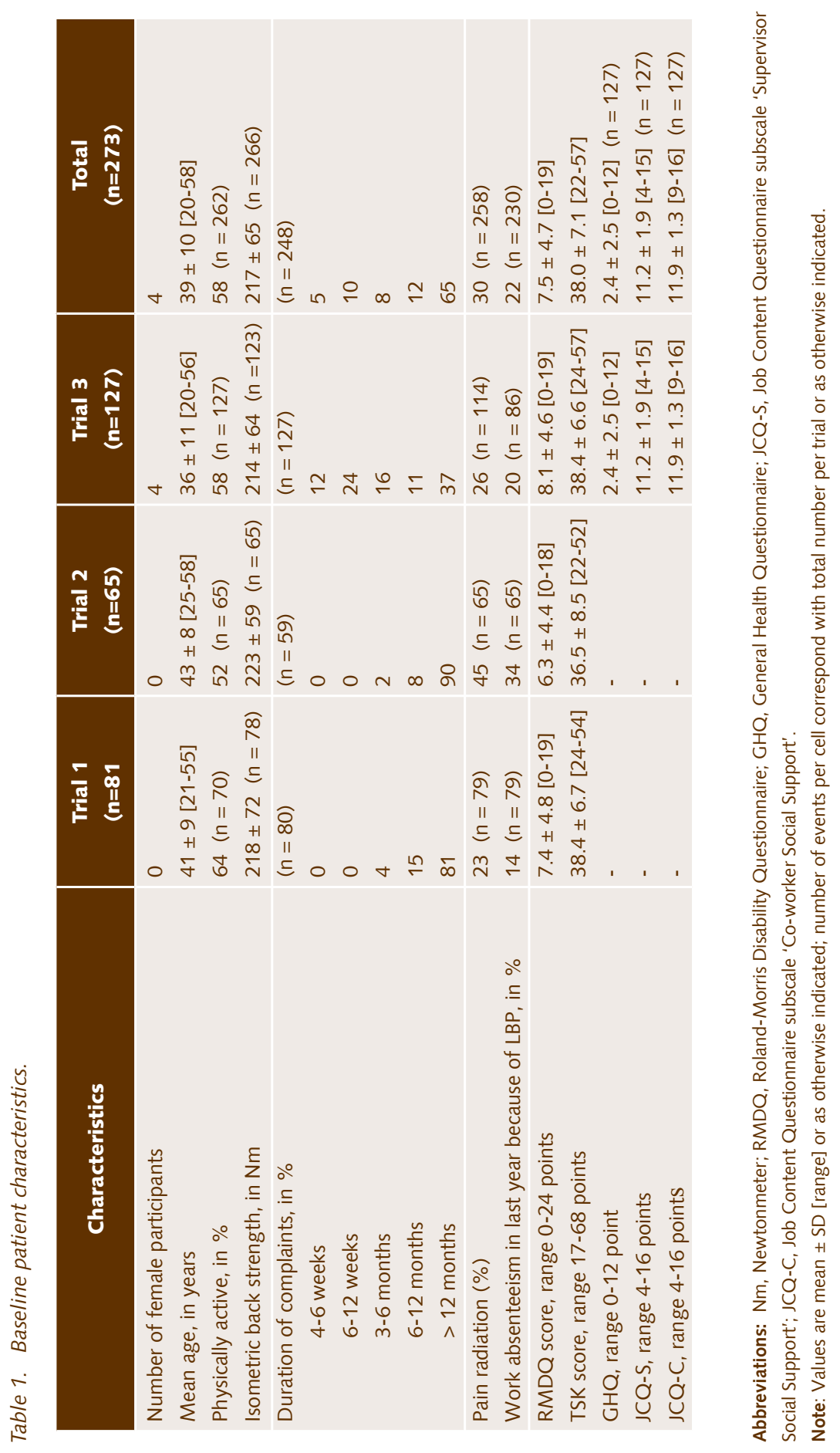




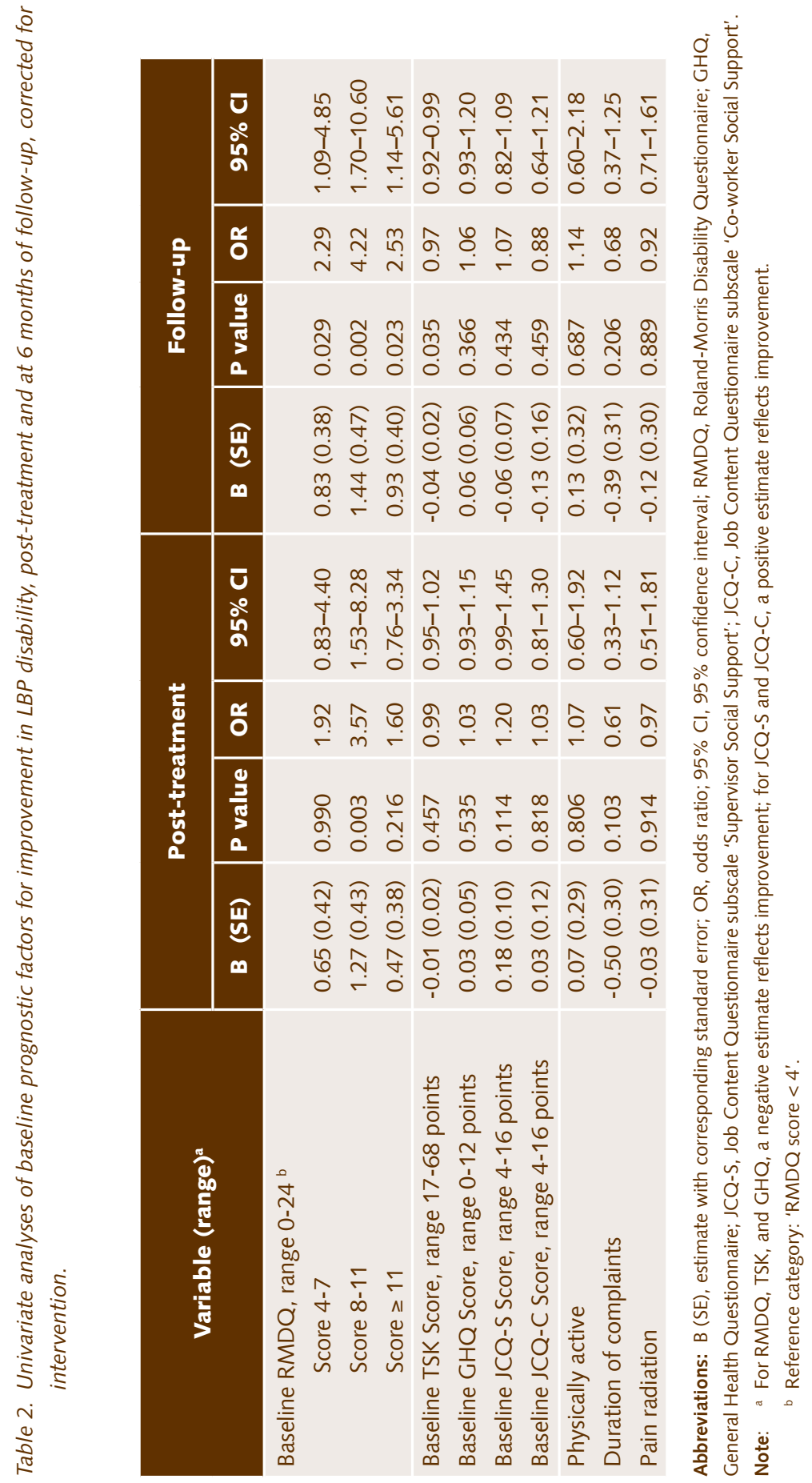




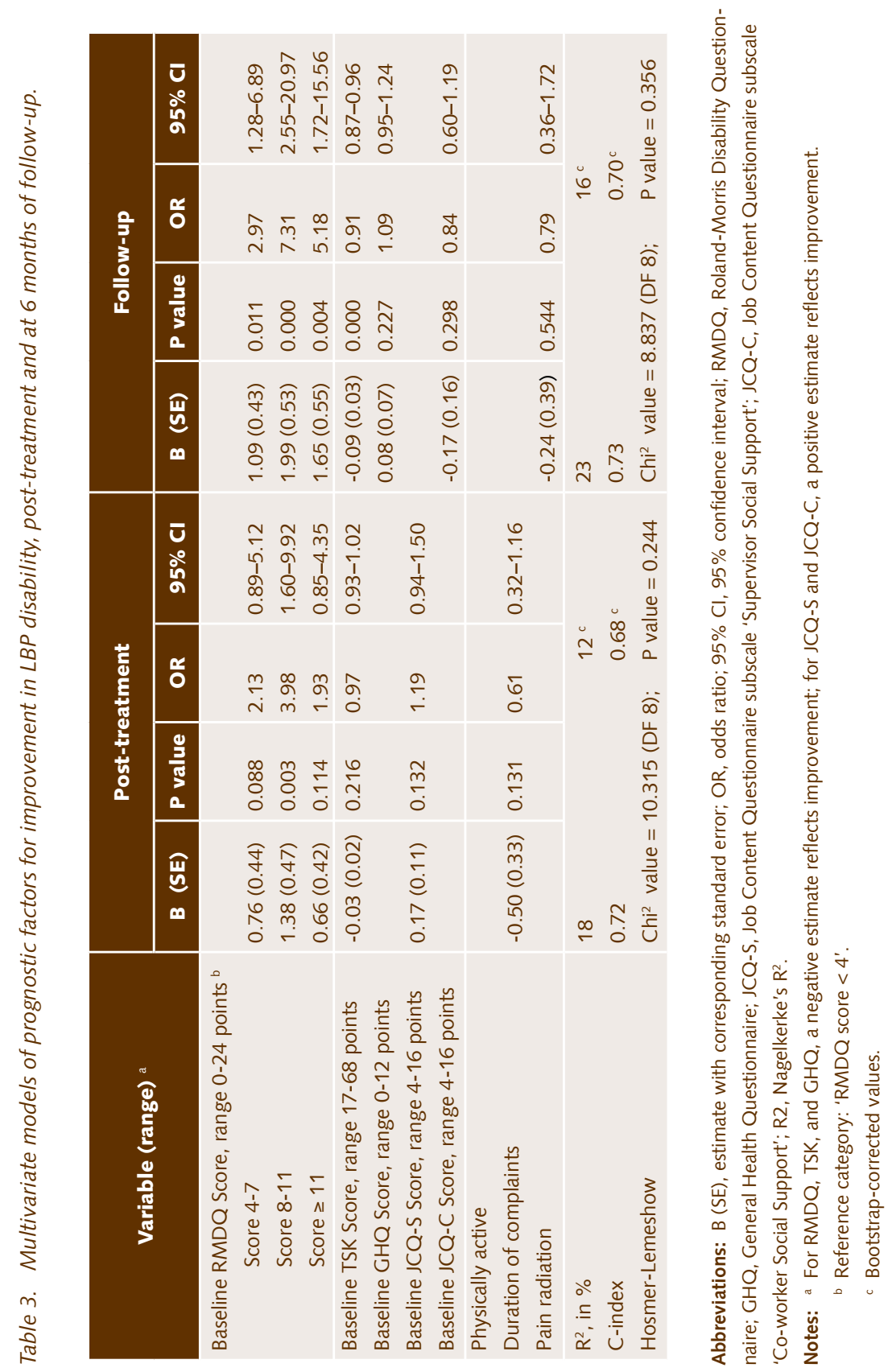


values ( $\geq 11$ ), had stronger prognostic value than low range values (4-7). Possibly, this finding can be explained by a 'law of diminishing returns' phenomenon, i.e., individuals with high levels of functional disability at baseline have a higher potential to improve then those with low disability levels. Nordin et $\mathrm{al}^{40}$ have reported that LBP patients with severe functional disability according to the Oswestry Disability Index were found to return to work later, possibly due to greater episode severity and/or a higher perception of being 'sick'. The fact that functional disability was our strongest prognostic factor was not a surprising finding, since changes in RMDQ values were part of the compound variable (improved versus not improved) we constructed as our outcome measure. But also in other studies, using different outcomes (e.g., work compensation status, time to return to work), baseline functional disability appeared to have prognostic value. ${ }^{16,61}$ We have chosen an outcome measure that was partly based on the RMDQ, since we consider its dimension 'functional disability due to perceived back pain' as more relevant for our mainly chronically injured, working population than indicators of the symptom itself, i.e., the degree of back pain. The treatments in our trials were predominantly aimed at restoring (physical) functioning, not at reducing the pain.

Our results support the conclusion of others that fear of movement has prognostic value in long-term disability. ${ }^{17,19,44,56} \mathrm{~A}$ positive influence of exercise on fear of movement behaviour has been reported in the literature., ${ }^{4,11}$ Exercises with fear-avoidance-based principles are thought to give additional benefits for subgroups of LBP patients with high levels of fear-avoidance beliefs. ${ }^{33,58}$ All three intervention modalities that were used in our trials consisted of exercise training, but none of them included specific fear-avoidance strategies. Our analyses suggest that fear-avoidance issues could have additional value when designing exercise treatment modalities in a LBP population that is already relatively physically active despite the presence of back pain.

Other factors in our models, which were less strongly associated with the outcome, were supervisor social support (short-term), duration of complaints (short-term), psychological distress (long-term), co-worker social support (longterm), and pain radiation (long-term). The fact that supervisor social support 
was associated to short-term LBP improvement, may very well be a reflection of the strong decisive influence of superiors in our hierarchical military organization on the participation of soldiers in duty time in activities such as a our research program. We used different definitions of duration of complaints in our trials (current complaints versus time since first LBP episode), all reflecting a different dimension of the history of the LBP symptoms. From the literature we know that a previous history of LPB is highly predictive of persistent symptoms, suggesting that people with one or more previous episodes are likely to have future multiple episodes. ${ }^{63}$ Psychological distress was another long-term factor in our model. Psychological distress has been recognized as a prognostic factor for both the onset and recurrence of LBP in many different populations, ranging from healthy young or middle-aged adults ${ }^{14,35}$ to patients in primary care settings $s^{22,34,57}$ and to individuals with work-related back injuries. ${ }^{9,16}$ In line with our study findings, Gheldof et $\mathrm{al}^{19}$ found that pain radiation was a significant risk factor for the development of long-term LBP in a population of predominantly male industrial workers. This risk was reduced by social support of co-workers.

Physical activity was the only factor that was removed from our regression models. There is inconsistent evidence in the literature about whether physically active leisure time versus sedentary activity have influence on the development of musculoskeletal morbidity in general, and LBP in particular. ${ }^{28}$ Some recent studies show associations between chronic LBP and physical activity or aerobic fitness level, respectively, ${ }^{38,53}$ but others do not. ${ }^{46}$

In general, we were able to generate well-fitted but rather weak-performing prognostic models, with percentages of explained variance (12-16\%) that are comparable or somewhat less favourable to those of other prognostic models in the same field of research $2,10,18,19,54,58,60$ may partly be explained by the fact that other than the chosen prognostic indicators, such as pain severity or job satisfaction, may play an important role in our population. However, in most cohort studies on nonspecific LBP, baseline factors only account for moderate amounts of variance in LBP outcomes, typically around 30\%. ${ }^{45}$ The large unexplained variances are, thus, most likely a reflection of the fact that an individual's course of nonspecific LBP is highly complex, affected by interacting factors that probably 
cover the whole spectrum of the bio-psychosocial model of pain and disability and that present themselves in different time phases of the process. ${ }^{62}$

There are some limitations in our study that must be recognized. First, our findings need to be interpreted with some caution, because the analyses were based on data from randomized clinical trials. These trials were initially not designed to identify prognostic factors for LBP improvement. The merged dataset may, therefore, still have insufficient power to detect these prognostic factors, despite our efforts to prevent overfitting problems by matching the number of potential prognostic factors to the number of outcome events. However, the confidence intervals of all but the dummy variables (RMDQ, intervention) were small, indicating that our study findings are robust. Second, due to the fact that one of the trials had a relatively short follow-up period, we were not able to measure effects at longer than 6 months after treatment. Moreover, in neither of the studies we used a true control group that could have reflected the natural course of LBP. In other words, all participants had experienced exercise therapy through our research program in the previous year and should be regarded as such when extrapolating the results of this study to other populations. This brings us to the external validity of our results. The population under study (male soldiers) is clearly not a representation of those presenting most commonly in primary care. Obviously, one should be careful in generalizing the results of this study to other samples, such as white-collar or female workers.

The population under study can be seen as a rather homogeneous group of individuals, consisting of young and middle-aged male subjects in their active working period ( $87 \%$ between $25-55$ years of age) who are not overly workdisabled by their condition despite incidentally reported high disability scores. In this respect, potential confounding influences of individual patient factors and work-related factors that have not been addressed in our analyses are expected to be low, which can be seen as a strength of our study. Moreover, we included a number of variables that have been identified as potentially important prognostic factors for LBP in several relevant areas (individual, work-related psychosocial, psychological), and that were measured with standardized, validated instruments. Finally, by using state-of-the-art statistical techniques for model 
building and evaluation of model performance, we have tried to prevent substantial shortcomings that have frequently been addressed in the use and report of logistic regression analyses in medical research, such as overfitting, assumption checks, and unrestricted use of automated variable selection. ${ }^{7}$

Not all trials collected the same prognostic factors. Consequently, after pooling the data, the percentage of missing data in three variables was around $50 \%$. For these situations, multiple imputation produces valid results under the MAR assumption, i.e., missings can be explained by the available data in the dataset since they are not dependent on the values of the variables themselves. This latter statement holds in our study because there was no specific reason in each of the trials to exclude variables. Furthermore, most variables in our study have shown to be important in low back pain prognosis, which means that all variables could be used in the imputation model to estimate the missing values. Several studies have shown that with multiple imputation valid results can be obtained when around $50 \%$ of the data is missing. ${ }^{21}$

\section{Conclusions}

This study implies that it can be of use for clinical practitioners to gather pretreatment patient information, in particular on individual levels of functional disability and fear-avoidance behaviour, in patient populations with characteristics comparable to ours (e.g., predominantly male, physically active, working, moderate but chronic back problems). By doing so, individuals at risk for poor long-term LBP recovery, i.e., individuals with high initial level of disability and prominent fear-avoidance behaviour, can be distinguished that may need additional cognitive-behavioural treatment. Further research is warranted to find out if this strategy actually leads to a higher improvement rate. 


\section{References}

1. Bagley $\mathrm{SC}$, White $\mathrm{H}$, Golomb BA. Logistic regression in the medical literature: standards for use and reporting, with particular attention to one medical domain. J Clin Epidemiol 2001;54(10):979-85.

2. Bekkering GE, Hendriks HJ, van Tulder MW, et al. Prognostic factors for low back pain in patients referred for physiotherapy: comparing outcomes and varying modeling techniques. Spine 2005;30(16):1881-6.

3. Beurskens AJ, de Vet HC, Koke AJ. Responsiveness of functional status in low back pain: a comparison of different instruments. Pain 1996;65(1):71-6.

4. Brox Jl, Sorensen R, Friis A, et al. Randomized clinical trial of lumbar instrumented fusion and cognitive intervention and exercises in patients with chronic low back pain and disc degeneration. Spine 2003;28(17):1913-21.

5. Cairns MC, Foster NE, Wright C. Randomized controlled trial of specific spinal stabilization exercises and conventional physiotherapy for recurrent low back pain. Spine 2006;31(19):E670-81.

6. Carey TS, Garrett JM, Jackman AM. Beyond the good prognosis. Examination of an inception cohort of patients with chronic low back pain Spine 2000;25(1):115-20.

7. Coste J, Lefrancois $G$, Guillemin F, et al. Prognosis and quality of life in patients with acute low back pain: insights from a comprehensive inception cohort study. Arthritis Rheum 2004;51(2):168-76.

8. Crook J, Milner R, Schultz IZ, et al. Determinants of occupational disability following a low back injury: a critical review of the literature. J Occup Rehabil 2002;12(4):277-95.

9. Crook J, Moldofsky H, Shannon H. Determinants of disability after a work related musculetal injury. J Rheumatol 1998;25(8):1570-7.

10. Dionne CE, Koepsell TD, Von Korff $M$, et al. Predicting long-term functional limitations among back pain patients in primary care settings. J Clin Epidemiol 1997;50(1):31-43.

11. Elfving B, Andersson T, Grooten WJ. Low levels of physical activity in back pain patients are associated with high levels of fear-avoidance beliefs and pain catastrophizing. Physiother Res Int 2007;12(1):14-24.

12. Enthoven P, Skargren E, Carstensen J, et al. Predictive factors for 1-year and 5-year outcome for disability in a working population of patients with low back pain treated in primary care. Pain 2006;122(1-2):137-44.

13. Ferreira ML, Ferreira PH, Latimer J, et al. Comparison of general exercise, motor control exercise and spinal manipulative therapy for chronic low back pain: A randomized trial. Pain 2007;131(1-2):31-7.

14. Feyer AM, Herbison P, Williamson AM, et al. The role of physical and psychological factors in occupational low back pain: a prospective cohort study. Occup Environ Med 2000;57(2):116-20.

15. Field A. Discovering statistics using SPSS. London: SAGE Publications Ltd, 2005.

16. Fransen $M$, Woodward $M$, Norton $R$, et al. Risk factors associated with the transition from acute to chronic occupational back pain. Spine 2002;27(1):92-8.

17. Fritz JM, George SZ. Identifying psychosocial variables in patients with acute work related low back pain: the importance of fear-avoidance beliefs. Phys Ther 2002;82(10):973-83. 
18. George SZ, Fritz JM, Childs JD, et al. Sex differences in predictors of outcome in selected physical therapy interventions for acute low back pain. J Orthop Sports Phys Ther 2006;36(6):354-63.

19. Gheldof EL, Vinck J, Vlaeyen JW, et al. Development of and recovery from short- and long-term low back pain in occupational settings: a prospective cohort study. Eur J Pain 2007;11(8):841-54.

20. Goldberg DP, Williams P. A user's guide to the general health questionnaire. Windsor: NFER-Nelson, 1988.

21. Greenland S, Finkle WD. A critical look at methods for handling missing covariates in epidemiologic regression analyses. Am J Epidemiol 1995;142(12):1255-64.

22. Grotle M, Brox J, Glomsrod B, et al. Prognostic factors in first-time care seekers due to acute low back pain. Eur J Pain 2007;11(3):290-8.

23. Harrell FE Jr. Regression Modeling strategies. Berlin: Springer, 2001.

24. Harts $\mathrm{CC}$, Helmhout $\mathrm{PH}$, de Bie RA, et al. A high-intensity lumbar extensor strengthening program is little better than a low-intensity program or a waiting list control group for chronic low back pain: a randomised clinical trial. Aust J Physiothe 2008;54(1):23-31.

25. Helmhout PH, Harts CC, Staal JB, et al. Comparison of a high-intensity and a lowintensity lumbar extensor training program as minimal intervention treatment in low back pain: a randomized trial. Eur Spine J 2004;13(6):537-47.

26. Helmhout PH, Harts CC, Staal JB, et al. Rationale and design of a multicenter randomized controlled trial on a "minimal intervention" in Dutch army personnel with nonspecific low back pain [ISRCTN19334317]. BMC Musculoskelet Disord 2004;5(1):40.

27. Helmhout PH, Harts CC, Viechtbauer W, et al. Isolated Lumbar Extensor Strengthening Versus Regular Physical Therapy in an Army Working Population With Nonacute Low Back Pain: A Randomized Controlled Trial. Arch Phys Med Rehabil 2008;89(9):1675-1685

28. Hildebrandt VH, Bongers PM, Dul J, et al. The relationship between leisure time, physical activities and musculoskeletal symptoms and disability in worker populations. Int Arch Occup Environ Health 2000;73(8):507-18.

29. Hosmer D, Lemeshow S. A goodness-of-fit test for the multiple logistic regression model. Communications in Statistics 1980;A10:1043-69.

30. Jordan $K$, Dunn KM, Lewis $M$, et al. A minimal clinically important difference was derived for the Roland-Morris Disability Questionnaire for low back pain. J Clin Epidemiol 2006;59(1):45-52.

31. Karasek R, Brisson C, Kawakami N, et al. The Job Content Questionnaire (JCQ): an instrument for internationally comparative assessments of psychosocial job characteristics. J Occup Health Psychol 1998;3(4):322-55.

32. Kent PM, Keating JL. Can we predict poor recovery from recent-on set nonspecific low back pain? A systematic review. Man Ther 2008;13(1):12-28.

33. Klaber Moffett JA, Carr J, Howarth E. High fear-avoiders of physical activity benefit from an exercise program for patients with back pain. Spine 2004;29(11):1167-72; discussion 1173.

34. Langworthy JM, Breen AC. Psychosocial factors and their predictive value in chiropractic patients with low back pain: a prospective inception cohort study. Chiropr Osteopat 2007;15:5. 
35. Linton SJ. Do psychological factors increase the risk for back pain in the general population in both a cross-sectional and prospective analysis? Eur J Pain 2005;9(4):355-61.

36. Mannion AF, Muntener M, Taimela S, et al. A randomized clinical trial of three active therapies for chronic low back pain. Spine 1999;24(23):2435-48.

37. Miller RP, Kori SH, Todd DD. The Tampa Scale. 1991.

38. Morken T, Mageroy N, Moen BE. Physical activity is associated with a low prevalence of musculoskeletal disorders in the Royal Norwegian Navy: a cross sectional study. BMC Musculoskelet Disord 2007;8:56.

39. Nachemson AL, Jonsson E. Neck and back pain: The scientific evidence of causes, diagnosis and treatment. Philadelphia: Lippincott Williams \& Wilkins, 2000

40. Norton $M$, Skovron $M$, Hiebert $R$, et al. Early predictors of delayed return to work in patients with low back pain. J Musculoskelet Pain 1997;5(2):5-27.

41. Pate RR, Pratt M, Blair SN, et al. Physical activity and public healt. A recommendation from the Center for Disease Control and Prevention and the American College of Sports Medicine. JAMA 1995;273:402-7.

42. Peduzzi $P$, Concato J, Kemper $E$, et al. A simulation study of the number of events per variable in logistic regression analysis. J Clin Epidemiol 1996;49(12):1373-9.

43. Pengel LH, Herbert RD, Maher CG, et al. Acute low back pain: systematic review of its prognosis. BMJ 2003;327(7410):323.

44. Picavet HS, Vlaeyen JW, Schouten JS. Pain catastrophizing and kinesiophobia: predictors of chronic low back pain. Am J Epidemiol 2002;156(11):1028-34.

45. Pincus $T$, Santos R, Breen A, et al. A review and proposal for a core set of factors for prospective cohorts in low back pain: a consensus statement. Arthritis Rheum 2008;59(1):14-24.

46. Rasmussen-Barr E, Lundqvist L, Nilsson-Wikmar L, et al. Aerobic fitness in patients at work despite recurrent low back pain: a cross-sectional study with healthy age- and gendermatched controls. J Rehabil Med 2008;40(5):359-65.

47. R Development Core Team. R: A language and environment for statistical computing. R Foundation for Statistical Computing, Vienna, Austria. ISBN 3-900051-07-0. Available at: http://www.R-project.org. Accessed July 18, 2008.

48. Roelofs J, Goubert L, Peters ML, et al. The Tampa Scale for Kinesiophobia: further examination of psychometric properties in patients with chronic low back pain and fibromyalgia. Eur J Pain 2004;8(5):495-502.

49. Roland M, Morris R. A study of the natural history of back pain. Part I: development of a reliable and sensitive measure of disability in low back pain. Spine 1983;8(2):141-4.

50. Rubin DB. Multiple imputation for nonresponse in surveys. New York: John Wileys \& Sons, 1987.

51. Schafer JL. Multiple imputation: a primer. Stat Methods Res 1999;8(1):3-15.

52. Shaw WS, Pransky G, Fitzgerald TE. Early prognosis for low back disability: intervention strategies for health care providers. Disabil Rehabil 2001;23(18):815-28.

53. Smeets RJ, Wittink $H$, Hidding A, et al. Do patients with chronic low back pain have a lower level of aerobic fitness than healthy controls?: are pain, disability, fear of injury, working status, or level of leisure time activity as sociated with the difference in aerobic fitness level? Spine 2006;31(1):90-7; discussion 98. 
54. Steenstra IA, Koopman FS, Knol DL, et al. Prognostic factors for duration of sick leave due to low-back pain in dutch health care professionals. J Occup Rehabil 2005;15(4):591-605.

55. Steyerberg EW, Eijkemans MJ, Harrell FE, Jr., et al. Prognostic modeling with logistic regression analysis: in search of a sensible strategy in small data sets. Med Decis Making 2001;21(1):45-56.

56. Swinkels-Meewisse IE, Roelofs J, Verbeek AL, et al. Fear of movement/(re)injury, disability and participation in acute low back pain. Pain 2003;105(1-2):371-9.

57. Thomas E, Silman AJ, Croft PR, et al. Predicting who develops chronic low back pain in primary care: a prospective study. Bmj 1999;318(7199):1662-7.

58. Underwood MR, Morton V, Farrin A. Do baseline characteristics predict response to treatment for low back pain? Secondary analysis of the UK BEAM dataset [IS RCTN32683578]. Rheumatology (Oxford) 2007;46(8):1297-302.

59. Van Buuren S, Oudshoorn K. Flexible multivariate imputation by MICE. In: Technical Report TNO Quality of Life. Leiden, The Netherlands, 1999.

60. Van der Hulst M, Vollenbroek-Hutten MM, Groothuis-Oudshoorn KG, et al. Multidisciplinary rehabilitation treatment of patients with chronic low back pain: a prognostic model for its outcome. Clin J Pain 2008;24(5):421-30.

61. Van der Weide WE, Verbeek JH, Salle HJ, et al. Prognostic factors for chronic disability from acute low-back pain in occupational health care. Scand J Work Environ Health 1999;25(1):50-6.

62. Waddell G. The back pain revolution. London: Churchill Livingstone, 1998.

63. Waddell G, Burton AK. Occupational health guidelines for the management of low back pain at work: evidence review. Occup Med (Lond) 2001;51(2):124-35.

64. Wendel-Vos GC, Schuit AJ, Saris WH, et al. Reproducibility and relative validity of the short questionnaire to assess health-enhancing physical activity. J Clin Epidemiol 2003;56(12):1163-9.

65. Wood AM, White IR, Royston P. How should variable selection be performed with multiply imputed data? Stat Med 2008;27(17):3227-46. 


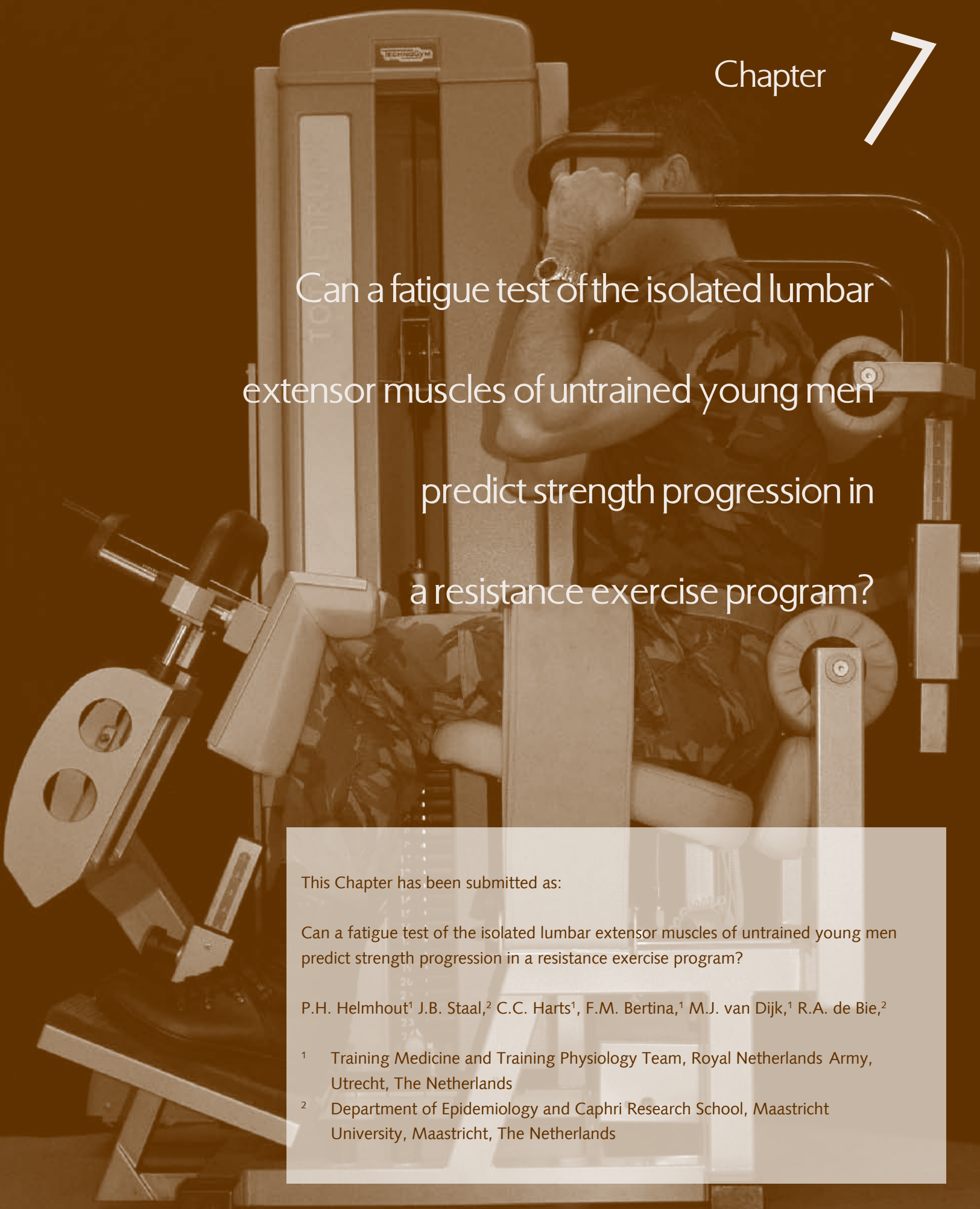




\section{Abstract}

Baseline performances on exercise tests until fatigue may have predictive value for individual training progression. We have designed a three-phased fatigue test of the lumbar extensor muscles, consisting of two consecutive measurements of full-range isometric back strength on a lumbar measurement device, separated by a dynamic back extension set to volitional fatigue. Differences between the strength values of the $1^{\text {st }}$ and $3^{\text {rd }}$ step is thought to indirectly reflect individual lumbar muscle fiber composition. The aim of this exploratory study was to investigate the predictive value of this "fatigue test" of the lumbar extensor muscles for training progression in a group of 28 healthy but predominantly sedentary male students, in an 8-week resistance exercise program. The program was primarily aimed at improving lumbar extensor endurance, by using a relative high number of repetitions and low training loads. Linear regression analysis was used to evaluate the relationship between lumbar strength progression and several fatigue test parameters. The main fatigue indicator in our regression models (isometric strength decline between $1^{\text {st }}$ and $3^{\text {rd }}$ step) did not show predictive value in lumbar strength progression in training and testing, respectively. On the other hand, the work capacity that subjects delivered in the dynamic set ( $2^{\text {nd }}$ step) had some predictive value. Based on these results, isometric strength decline measurement has no additional value to a standardized set of repetitions until failure in predicting future training performance. In practice, this means that a lower back training machine could be used at baseline to assist in tailoring individual lumbar training regimes, without the additional use of an isometric-strength testing module. 


\section{Introduction}

Resistance exercise can lead to adaptations of many different physiological systems, the most obvious ones being progression in muscular strength and muscular endurance. Together with size and number of the muscle fibers involved, skeletal muscle fiber type composition is thought to play a vital role in the level of strength progression in resistance exercise. ${ }^{1}$ It has been suggested that targeting training regimes to individual muscle fiber-type profiles may lead to higher training-induced muscle endurance and/or strength gains. ${ }^{2,3}$ For example, individual muscles or muscle groups with a relative predominance of slow-twitch fibers may adapt better to resistance exercise using many repetitions (reps) and low training loads. On the other hand, resistance exercise using fewer reps with a heavy weight may be a more effective training stimulus for muscles with a greater proportion of fast-twitch fibers. In other words, it can be hypothesized that training within the individual's repetition variability, i.e., based on largely genetically determined fiber type characteristics, may lead to a higher training efficiency.

The literature is yet scarce and inconclusive about this concept of using 'individualized' training stimuli according to muscle fiber composition. This may have to do with the practical difficulties that come with assessing fiber composition. The only way to directly determine fiber composition is to take invasive muscle biopsies with a hollow needle, but this method has several disadvantages (e.g., biopsy sampling artefacts; risk of side effects; high costs when used on larger scale). ${ }^{4}$

In training or rehabilitation, the 'output' of muscles as tested in non-invasive functional tests (e.g., maximal isokinetic strength, repetition variability, muscle fatigue parameters) may be a promising alternative to muscle fiber composition in optimizing exercise regimes to the individual's training potential. This may particularly be the case when the focus of interest is on utilizing information from all the muscles involved in a movement, rather than from one individual muscle. For example, it may be particularly difficult to produce a set of biopsies representative of the muscle fiber composition of the lumbar extensors, being a compound muscle group consisting primarily of the erector spinae and transversus spinae muscles. ${ }^{16}$ 
To our knowledge, the association between training progression of the lower back muscles and baseline performance measurements utilizing muscle fatigue and repetition variability characteristics has not been studied before. In our research efforts to design an effective treatment method for nonspecific low back pain in the Dutch military, we have developed an exercise machine for testing and training the lower back based on earlier concepts. ${ }^{5,6}$ In this concept, we isolate the lumbar extensors by means of pelvic fixation, and use multiple isometric measurement of back extension strength throughout the range of motion. In a technical report, Jones (A. Jones, personal communication, MedX Corporation, 1993) suggests a three-part procedure to determine individual fatigue characteristics of the lumbar extensors using a back exercise machine that is based on similar principles of muscle group isolation and multiple isometric testing. The first step in this 'fatigue test' is a full-range test of isometric back strength. The second step is a dynamic back extension exercise to volitional fatigue. Finally, remaining isometric strength is tested directly following the dynamic exercise. The proportion of strength decline between the first and second isometric test is an indication of muscle fatigue. A high level of fatigue is thought to reflect a relatively high percentage of fast-twitch fibers in the lumbar extensors, whereas little strength decline should represent a lower percentage of fast-twitch fitbers.

We are intrigued by the idea that individuals with relatively high or low levels of muscle fatigue measured in our fatigue test would respond predictably different to the same resistance exercise regime. The aim of this exploratory study was, therefore, to investigate the predictive value of a baseline three-phased fatigue test for training progression in an 8-week resistance exercise program in a group of healthy but predominantly sedentary male students. The exercise program was primarily aimed at improving lumbar extensor endurance, by using a relative high number of reps and low training loads. We hypothesized that subjects with low levels of fatigue on the baseline exercise test (possibly reflecting a low percentage of fast-twitch fibers) would show a trend towards larger training progression than subjects with high levels of fatigue, as they were expected to be loaded more appropriate to their physiological training potential. 


\section{Methods}

\section{Experimental approach to the problem}

This study was part of a larger training study in which the effects of an 8week resistance exercise regime predominantly aimed at whole-body maximal strength was measured in healthy, untrained young men. Our sub-study was exploratory, with the purpose of evaluating the predictive value for individual lumbar strength progression of baseline performance tests utilizing muscle fatigue characteristics of the individuals' lumbar extensors. This relationship has been suggested in the literature, but never scientifically documented ( $A$. Jones, personal communication, MedX Corporation, 1993). If this and other research confirm such an association, baseline performance tests could be developed that assist the design of training regimes tailored to individual lumbar muscle fiber type profiles.

\section{Subjects}

Twenty-eight young men were recruited from a population of male students from Maastricht University, The Netherlands. Exclusion criteria were: age younger than 18 and older than 35 years; hypertension (SBP > $140 \mathrm{mmHG}$, DBP > $90 \mathrm{mmHG}$ ); orthopaedic surgery in the last 3 years; medication and/or neuromuscular or orthopaedic disorders that contraindicated resistance exercise; experience with progressive resistance exercise training.

Table 1 shows baseline characteristics of the included subjects. After explanation of the study demands, written informed consent was obtained from all participants. The study protocol was approved by the Medical Ethical Committee of Maastricht University.

\section{Procedure and Equipment}

The resistance exercise program consisted of 8 weeks of training, twice a week, preceded by a fatigue test (see below). A second fatigue test was performed at the end of the program. Training sessions and fatigue tests were both carried out on specialized lower back machines (training: OriGene Revival System ${ }^{\circledR}$, OriGene Inc, The Netherlands; testing: Total Trunk Rehab ${ }^{\circledR}$, Technogym Inc, 
Italy). These lower back devices are equipped with a knee-lock system and a thigh-restraining belt to immobilize both hips and thighs, allowing the participant only to move the isolated lumbar extensors from flexion to extension and back. Moreover, the machine arm can be fixed in several angles and the force that subject exerts to this fixed arm can be transferred through a force sensor to a personal computer by means of a force transducer (Digimax RS 232, Interface Mechatronic Inc., Germany). A more detailed description of the test/training devices used can be found elsewhere. ${ }^{5,6}$

Table 1. Baseline subject characteristics $(n=28)$.

\begin{tabular}{|c|c|c|c|}
\hline Characteristics & \multicolumn{3}{|c|}{ Baseline values a } \\
\hline Age, in years & 20.6 & \pm 1.3 & [19-24] \\
\hline Height, in $\mathrm{cm}$ & 186 & \pm 5 & [178-195] \\
\hline Weight, in $\mathrm{kg}$ & 77.7 & \pm 7.3 & {$[64.5-93.0]$} \\
\hline Fat-free mass, in \% & 82.4 & \pm 4.3 & {$[74.5-88.5]$} \\
\hline SQUASH sports active score, in minutes per week ${ }^{b}$ & 176 & \pm 113 & {$[10-360] \quad(n=27)$} \\
\hline Absolute difference in back strength fatigue test, in $\mathrm{Nm}$ & 718 & \pm 481 & {$[9-1836]$} \\
\hline Work capacity fatigue test (number of reps $x$ weight), in $\mathrm{kg}$ & 629 & \pm 171 & {$[300-1024] \quad(n=27)$} \\
\hline Work capacity training (number of reps $x$ weight), in $\mathrm{kg}^{\mathrm{c}}$ & 1760 & \pm 417 & {$[980-2850]$} \\
\hline
\end{tabular}

Abbreviations: SQUASH, Short Questionnaire to Assess Health Enhancing Physical Activity; Nm, Newtonmeter reps, repetitions.

Notes: a Values are mean \pm SD [range] or as otherwise indicated; number of events per cell corres pond with total number or as otherwise indicated.

b Mean reference values for Dutch boys/men aged 15-25 yrs in 2004-2007: $308 \pm 12$ min per week of sports activities.

c Calculated as mean of two sets in 3rd training.

Height and body weight of each subject were measured at baseline, as well as the percentage body fat (from which the individual's fat-free mass was derived). Body fat was assessed using skinfold measurements (Servier Nederland BV) from four sites: biceps, triceps, subscapular, and supra-iliac skinfold. ${ }^{7}$ Both at baseline and at the end of the training program, the level of sports activity was measured with one item from the Short Questionnaire to Assess Health Enhancing Physical Activity. ${ }^{8}$ Regular dietary intake before and after the training program was measured using a validated semi quantitative food frequency questionnaire. ${ }^{9}$ 


\section{Training Protocol}

The 8-week exercise regime of the lumbar extensors was predominantly aimed at muscular endurance: two sets of 15 to 20 reps, with 1 minute rest in between, were performed on the lower back machine in each training session. This training zone is equivalent to approximately $50 \%$ to $70 \%$ of the one-repetition maximum (1-RM), respectively. If the subject was able to perform more than 20 reps of slow and controlled back extension and flexion movements, $2.5 \mathrm{~kg}$ weight was added in the next training set or training session, respectively. Vice versa, if the subject performed below the training zone, the weight was lowered with $2.5 \mathrm{~kg}$ the next time. This training protocol is based both on existing protocols $^{10,11}$ and on our own clinical experiences. All training sessions were conducted by one test leader, who paid special attention to the individual's execution of the training in terms of movement (hollowing and flattening of the lumbar lordosis), pace (2 s extension; $3 \mathrm{~s}$ flexion), and range (full range). The training load used and the number of reps completed during each training session were recorded.

\section{Fatigue Test}

After a standardized warming-up, i.e., 5 min on a bicycle ergometer at a predetermined pace, followed by performing a few reps at increasing loads on the lower back machine, the first step in our three-part fatigue test protocol was a multiple measurement of isometric back strength in five fixed positions of the machine arm: $55^{\circ}$ (full flexion), $40^{\circ}, 25^{\circ}, 10^{\circ}$ and $-5^{\circ}$ (full extension) relative to the vertical. In these measurements, corrections were made for the effect of gravity on the mass of the involved body parts and by stored energy (e.g. compression of abdominal tissues). Thus, net muscular torques can be calculated from the functional torques in every angle and these values can be averaged to a mean maximal isometric strength value.

After a standardized recovery period, i.e., 5 min of light pedalling on a bicycle ergometer, the second step in our fatigue test was a dynamic set until failure at a weight stack load corresponding to approximately 50\% (extension) and 60\% (flexion) of the mean individual isometric strength measured in step one. This dynamic movement was paced at a slow and controlled speed: 2 seconds 
for back extension, 3 seconds for flexion. We have empirically found that most healthy individuals are slightly weaker in the more extended parts and, therefore, we used a degressive load towards back extension (P.H. Helmhout, unpublished data, September, 1998). An in-house software program calculated this load from the individuals' isometric strength curve.

Directly following the dynamic set, subjects were asked to repeat the same multiple isometric strength test from the first step. Differences between the strength values of the $1^{\text {st }}$ and $3^{\text {rd }}$ step was used as an indicator of back muscle fatigue.

A previous test-retest reliability study showed that reproducibility was lower between a fatigue test that was performed by an individual for the first time and a second fatigue test, than between a second and third test, possibly due to a learning effect (P.H. Helmhout, unpublished data, January 2002). For this reason, we planned a trial fatigue test in the week before the baseline measurement, enabling each participant to familiarize with the test procedure.

At the end of the 8-week training period, all measurements including the fatigue test were repeated.

\section{Statistical Analyses}

Linear regression analysis was used to evaluate the relationship between lumbar strength progression during the 8-week resistance training program and several muscle performance indicators at baseline. We defined 'lumbar strength progression' in two different ways. First, the difference between mean isometric back strength measured in the $1^{\text {st }}$ step of the baseline fatigue test versus that of the post-training fatigue test was calculated. Second, the difference between work capacity measured in the $2^{\text {nd }}$ step of the baseline fatigue test versus that of the post-training fatigue test was calculated. (Work capacity was calculated as weight on the weight stack multiplied by number of reps.)

Isometric and dynamic strength progression were included in the regression analyses as the outcome variables. The difference between mean isometric strength values of the $1^{\text {st }}$ and $3^{\text {rd }}$ step of the baseline fatigue test (our 'fatigue indicator') 
was used as a potential predictor in the regression models, as well as the work capacity at the start of the training program. Pre-training work capacity was calculated as the average of the two sets in the $3^{\text {rd }}$ training session (second week), since the first two training sessions were mainly used for subjects to familiarize with both protocol and training device. Work capacity produced in the $3^{\text {rd }}$ training session, percentage of fat-free mass at baseline, and pre-post differences in sports activity score, respectively, were also included in the regression models as 'correcting factors', assuming that these factors might influence the association between our potential predictors and the outcome.,12 To meet the rough guideline in regression model building of minimal 10 events per independent variable, ${ }^{10}$ we could only include 3 variables per model $(n=28)$. Therefore, we built three models per dependent variable with each containing the two predictors and one of the three correcting factors.

Occasional missing values (one missing value in activity score; one missing value in work capacity initial fatigue test) were substituted by their group mean. All variables were entered in the model followed by backward elimination retaining only the strongest factors at a liberal $P$ value $(P<.50)$, following recommendations by Steyerberg et al. ${ }^{13}$

The adjusted $R^{2}$ was used as a measure of the predictive power of the model, indicating the percentage of variation in the outcome explained by the model. We used residual regression diagnostics provided by the software package to assess the goodness-of-fit of each model; collinearity diagnostics was used to check if factors were highly correlated. ${ }^{14}$ All analyses were performed using SPSS for Windows Version 15.0.

\section{Results}

Table 2 shows the overall strength progression of our study group during the 8-week training period. Isometric lumbar strength, as measured in the $1^{\text {st }}$ step of the fatigue test, improved with, on average, $400 \mathrm{Nm} \pm 427 \mathrm{Nm}$. Dynamic lumbar strength, calculated as the work capacity in the $2^{\text {nd }}$ step of the fatigue test, decreased over time with, on average, $47 \mathrm{~kg} \pm 178 \mathrm{~kg}$ (mean pre-post dif- 
ference in number of reps: $-4.5 \pm 7.3$; mean pre-post difference in training load: $25 \mathrm{~kg} \pm 21 \mathrm{~kg}$ ). Dynamic lumbar strength, calculated as the work capacity (number of reps $x$ training load) delivered in the training sessions improved with, on average, $584 \mathrm{~kg} \pm 558 \mathrm{~kg}$ (mean pre-post difference in number of reps: $1.6 \pm$ 5.9; mean pre-post difference in training load: $11.9 \mathrm{~kg} \pm 6.3 \mathrm{~kg}$ ).

Table 2. Mean lumbar stength during 8 weeks training program.

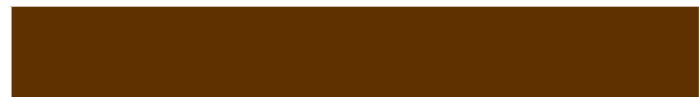

Mean \pm SD [range]

Isometic strength progression (in $\mathrm{Nm}$ ) ${ }^{\mathrm{a}}$ $400 \pm 427[-621 ; 1197]$

Dynamic strength progression fatigue test (in $\mathrm{kg})^{\mathrm{b}}$ $-47 \pm 178[-514 ; 242]$ Dynamic strength progression training (in $\mathrm{kg})^{\mathrm{c}}$ $584 \pm 558[-575 ; 1910]$

Abbreviations: SD, standard deviation; Nm. Newtonmeter; Kg, kilogram.

Notes: a Isometric strength progression, defined as pre-post differece in mean isometric lumbar strength in 5 positions, measured in $1^{\text {st }}$ step, of fatigue test.

b Dynamic strength progression (fatigue test), defined as pre-post difference in work capacity (no. of reps $x$ weight) measured in $2^{\text {nd }}$ step post-training fatigue test versus baseline fatigue test.

c Dynamic strength progression (training), defined as pre-post difference in work capacity (no. of reps $x$ weight) measured in last training session versus $3^{\text {rd }}$ training session.

Table 3 summarizes the results of the regression analyses. All three final regression models for the two outcomes contained only one significant predictor for lumbar strength progression at a significance level of $\alpha=.05$ : work capacity of the dynamic set $\left(2^{\text {nd }}\right.$ step) in the baseline fatigue test. This factor was moderately associated with both outcomes $(r=0.58-0.59)$. Our fatigue indicator, difference in back strength between the $1^{\text {st }}$ and $3^{\text {rd }}$ step of the initial fatigue step, was not significant in each of the models. The retained factors together explain $30-32 \%$ of the variation in outcome in the six models.

All models showed regression diagnostics within normal ranges, indicating adequate model fit. One model showed a potential influential case: rerunning the regression analysis without this case did not change the results substantially. Collinearity diagnostics indicated that the assumption of 'no multicollinearity' was met in all models. (Results not presented). 

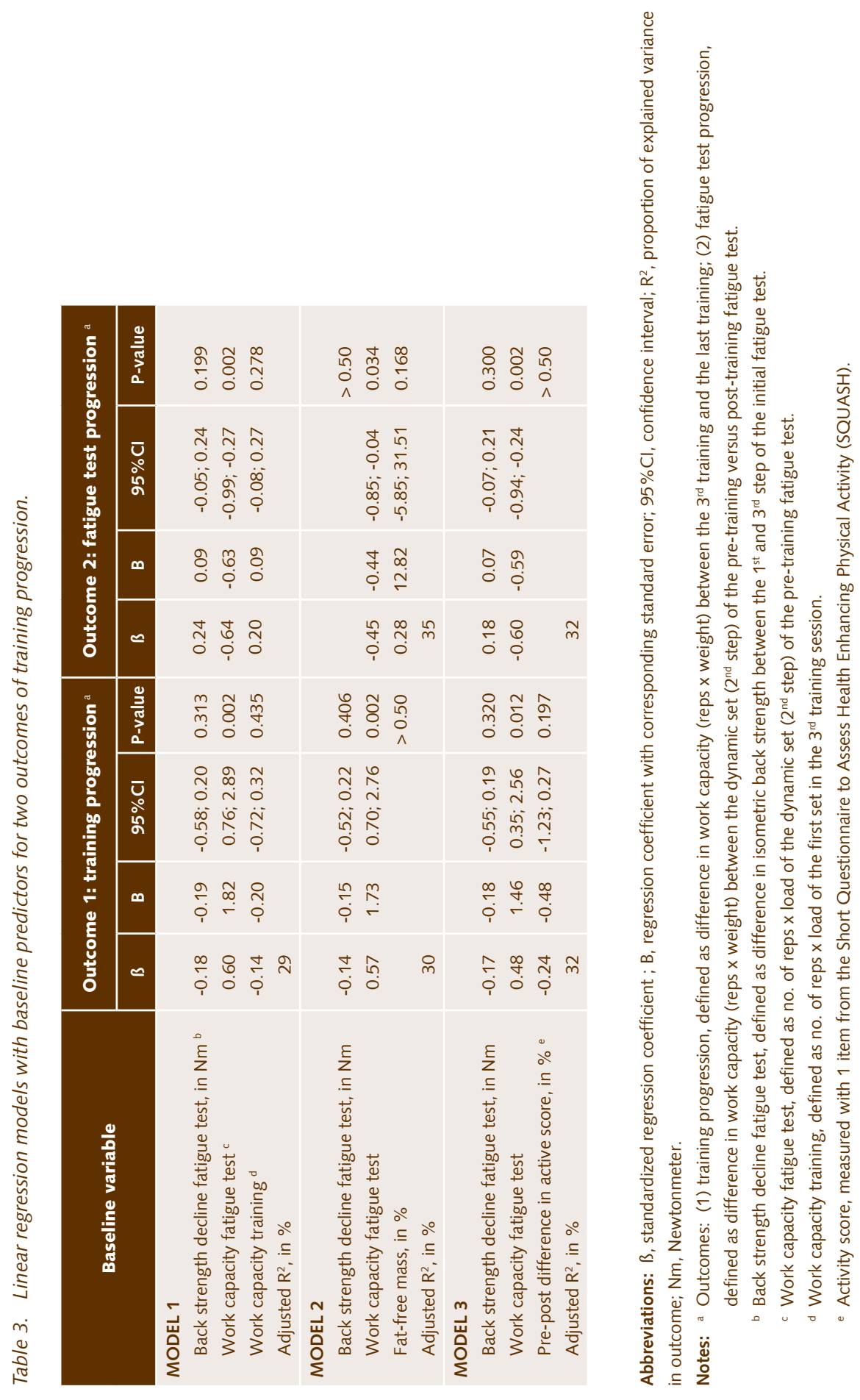


\section{Discussion}

The purpose of this exploratory study was to determine whether the performance on a lumbar fatigue test had predictive value for the level of strength progression in a lumbar extensor endurance training program, in a group of 28 untrained young men. We hypothesized that subjects with low levels of fatigue would show a trend towards larger lumbar strength progression than subjects with high levels of fatigue, as they were expected to be loaded more appropriate to their physiological training potential. Based on the results, this hypothesis is rejected. The fatigue indicator in our regression models, i.e., the proportion of strength decline between two successive isometric back strength tests separated by a dynamic set, did not show predictive value in lumbar strength progression. On the other hand, improvement of work capacity during the dynamic part of the baseline fatigue test, had some predictive value.

Several explanations can be given for the lack of predictive value of our lumbar fatigue indicator. First, lumbar muscle fatigue as measured in our fatigue test may have no clear relationship with fiber distribution in the lumbar muscles. We did not find a significant correlation between our fatigue indicator and 'fresh' isometric strength (corrected for muscle mass) derived from the $1^{\text {st }}$ step of our fatigue test (not shown). A correlation would have been in line with our hypothesis, in that stronger persons, with higher percentages of fast-twitch fibers, would show less training progression. Possibly, other factors than fiber type have a dominant influence on level of strength decline in our fatigue test (e.g., number and types of motor units involved, fiber length, pennation angle). ${ }^{15,16}$

A second theory to explain the lack of predictive value of our fatigue indicator is that the physiological stimulus of our 8-week training regime may have been inadequate or too short to differentiate enough between individuals with a greater and smaller proportion of fast-twitch fibers in the lumbar extensors, respectively. As we know from the literature, training sets that are performed until fatigue may very well lead to the recruitment of both slow-twitch fibers and fast-twitch fibers, irrespective of the fact that the training set is performed using few or many reps. 15 It is less likely that 8 weeks of training was too short a period of 
time to actually cause a physiological effects on the muscle fiber level, since 4 to 6 weeks of resistance training has been reported to be sufficient to attribute strength gains mainly to muscle fiber hypertrophy. ${ }^{17}$

Third, the outcome variables that have been chosen in our regression models may not adequately reflect strength progression during the training program. Our first outcome, pre-post changes in maximal isometric strength as measured in several positions throughout the lumbar range of motion, may not be a good representation of changes in lumbar strength during dynamic training. In the literature, isometric strength and endurance parameters have been found less suitable to infer the functional capacity of the musculature under dynamic conditions. ${ }^{1}$ We did not find a significant relationship between isometric strength decline and dynamic training performance in our analyses (not shown).

Our other indicator of strength progression, pre-post difference in work capacity delivered in the dynamic set of the fatigue test, may have another disadvantage: number of reps and training load are equally weighted in the calculation of work capacity, but the first can fluctuate over time to a much larger extent than the latter. Despite these possible inadequacies, the regression analyses showed consistent results for both outcome variables.

Finally, inter-individual variation in the distribution of slow-twitch fibers and fast-twitch fibers in the lumbar extensor muscles of the study group may simply not have been large enough to distinct good- and bad-responders on a training regime emphasizing muscle strength or endurance. The literature reports, nevertheless, on high inter-individual variation of both fiber types within the compound lumbar muscle group, most likely reflecting different activity patterns. ${ }^{3,16}$ Clearly, more research is indicated to further unravel the potential predictive value of muscle fatigue in determining individual strength progression in a training program.

The results indicate a potential predictive value for training progression of the dynamic set of repetitions until failure in the initial fatigue test. The number of reps until failure at a predetermined load (mostly expressed in \% 1-RM) has previously been reported to correlate with skeletal muscle fiber composition, ${ }^{18,19}$ 
although a recent study did not find any significant relationship between the two parameters..$^{20}$ To our knowledge, only one study has studied the predictive value of the number of reps until failure for strength progression in a resistance training program: Hickson et al19 have found correlations up to $r=0.83$ between number of reps at different exercise intensities $(40 \%, 60 \%$, and $80 \%$ of $1 R M$, respectively) in a parallel squat and slow-twitch fiber composition in the vastus lateralis muscle. Individuals with higher percentages of slow-twitch fibers showed the same relative training improvement at either of the exercise intensities as individuals with lower percentages of slow-twitch fibers. The statistical power of this study, however, was relatively low $(n=8)$.

Several potential confounding factors have been suggested in the literature to influence the number of reps at a given load: gender ${ }^{21}$ initial strength level, ${ }^{22}$ and the amount of muscle mass. ${ }^{23}$ Moreover, exercise history and the level of physical activity can alter fiber composition and other functional properties of individual muscles. ${ }^{12,16}$ For these reasons, we included fat-free mass, pre-post changes in physical activity, and work capacity level in the initial training phase as covariables in our regression analyses. Neither of these factors was significant in the final regression models and, therefore, their influence on the predictive value of our main indicators can be neglected in this study population. Dietary changes were not included in our analyses: except for alcohol consumption (11\% reduction), the intake of all other macronutrients (carbohydrates, proteins, saturated and unsaturated fat) did not change more than $3 \%$ during the 8 -week training program.

In general, we were able to generate adequately fitted but moderately performing regression models, accounting for 30 to $32 \%$ of the variance observed in training performance. We followed a single-testing strategy by using only information from fatigue properties of muscles involved. Based on their study results, Suter et $\mathrm{al}^{24}$ have plead for a multi-testing approach in which information from different mechanical properties of muscle fibers (e.g., contraction speed, peak power, fatigue resistance) yield better predictions of fiber type distribution. Indeed, their regression analyses produced relatively better model performance $\left(R^{2}=0.52\right)$, but this may very well be the result of model overfitting, conside- 
ring the low number of observations per predictor variable $(<3) .{ }^{25}$ The fact that only one-third of the total variance in our dataset could be explained by our models implies that other factors determine to a large extent the individual's ability to gain muscle strength and/or endurance through resistance training. These factors could be related to anthropometry (e.g., length of body parts, point of tendon insertion) or to psychological aspects (e.g., motivation to repeatedly perform until fatigue). Moreover, the quality of administrating each training session (e.g., slow and controlled movements, proper resting times in between sets) is likely to have a considerable impact on the training results, but we believe to have properly addressed this factor in our training protocol. All training sessions were supervised by one and the same fitness instructor. One of the reasons to choose a high-repetition, low-load training is that, in general, this protocol is perceived to be less difficult for untrained and inexperienced subjects than lifting comparatively heavier weights with fewer reps. ${ }^{26,27}$ We chose young and untrained subjects since they have a relatively high potential to respond to any type of resistance training protocol, particularly during the initial stages of training. ${ }^{17,22}$

In conclusion, we did not find evidence that a three-staged baseline fatigue test using a lumbar extension test and training machine is of value in individualizing training protocols for the lower back. However, a baseline set of reps until failure at a given load (derived from fresh isometric lumbar strength) showed some predictive value for training-induced strength development of the lumbar extensor muscles. In practice, this means that a lower back training machine could be used at baseline to assist in tailoring individual lumbar training regimes, without the additional use of an isometric-strength testing module. Future research is warranted to elucidate the optimal strategy for such a pre-training set of reps to discriminate individuals according to their training potential.

Acknowledgements: We thank the researchers from the Department of Human Movement Sciences of the Maastricht University for their assistance in the training program. We also thank the OriGene Corporation for using one of their OriGene Revival System ${ }^{\circledR}$ machines during the time of this study. The results of the present study do not constitute endorsement of the product by the authors. 


\section{References}

1. Murphy AJ, Wilson GJ. Poor correlations between isometric tests and dynamic performance: relationship to muscle activation. Eur J Appl Physiol Occup Physio 1996;73:353-57.

2. Karp JR. Muscle fiber types and training. Strength Cond J 2001;23:21-26.

3. Mannion AF, Dumas GA, Cooper RG, et al. Muscle fibre size and type distribution in thoracic and lumbar regions of erector spinae in healthy subjects without low back pain: normal values and sex differences. J Anat 1997;190:505-13.

4. Harris BA. The influence of endurance and resistance exercise on muscle capillarization in the elderly: a review. Acta Physiol Scand 2005;185:89-97.

5. Helmhout PH, Harts CC, Viechtbauer W, et al. Isolated Lumbar Extensor Strengthening Versus Regular Physical Therapy in an Army Working Population With Nonacute Low Back Pain: A Randomized Controlled Trial. Arch Phys Med Rehabil 2008;89:1675-85.

6. Helmhout PH, Harts CC, Staal BJ, et al. Comparison of a high-intensity and a lowintensity lumbar extensor training program as minimal intervention treatment in low back pain: a randomized trial. Eur Spine J 2004;13:537-547.

7. Durnin JV, Womersley J. Body fat assessed from total body density and its estimation from skinfold thickness: measurements on 481 men and women aged from 16 to 72 years. Br J Nutr 1974;32:77-97.

8. Wendel-Vis GC, Schuit AJ, Saris WH. Reproducibility and relative validity of the short questionnaire to assess health-enhancing physical activity. J Clin Epidemiol 2003;56:1163-9.

9. Grootenhuis PA, Westenbrink S, Sie CM, et al. A semiquantitative food frequency questionnaire for use in epidemiologic research among the elderly: validation by comparison with dietary history. J Clin Epidemiol 1995;48:859-68.

10. American College of Sports Medicine. ACSM's guidelines for exercise testing and prescription. Philadelphia: Lippincott Williams \& Wilkins, 2000.

11. Pollock ML, Leggett SH, Graves JE, et al. Effect of resistance training on lumbar extension strength. Am J Sport Med 1989;17:624-629.

12. Widrick JJ, Stelzer JE, Shoepe TC, et al. Functional properties of human muscle fibers after short-term resistance exercise training. Am J Physiol Regul Integr Comp Physiol 2002;283:R408-16.

13. Steyerberg EW, Eijkemans MJ, Harrell JR, et al. Prognostic modelling with logistic regression analysis: in search of a sensible strategy in small data sets. Med Decis Making 2001;21:45-56.

14. Field A. Discovering statistics using SPSS. 2nd ed. London: SAGE Publications Ltd, 2005.

15. Fleck SJ, Kraemer WJ. Designing Resistance Training programs. 2nd ed. Champaign, IL: Human Kinetics, 1997.

16. Ng JK-F, Richardson CA, Kippers $V$, et al. Relationship between muscle fiber composition and functional capacity of back muscles in healthy subjects and patients with back pain. JOSPT 1998;27:389-402.

17. Deschenes MR, Kraemer WJ. Performance and physiologic adaptations to resistance training. Am J Phys Med Rehabil 2002;81:S3-16. 
18. Douris PC, White BP, Cullen RR, et al. The relationship between maximal repetition performance and muscle fiber type as estimated by noninvasive technique in the quadriceps of untrained women. J Strength Cond Res 2006;20:699-703.

19. Hickson RC, Hidaka K, Foster C. Skeletal muscle fiber type, resistance training, and strength-related performance. Med Sci Sports Exerc 1994;26:593-598.

20. Terzis $G$, Spengos $K$, Manta $P$, et al. Fiber type composition and capillary density in relation to submaximal number of repetitions in resistance exercise. J Strength Cond Res 2008;22:845-50.

21. Sakamoto A, Sinclair PJ. Effect of movement velocity on the relationship between training load and the number of repetitions of bench press. J Strength Cond Res 2006;20:523-27.

22. Crewther B, Cronin J, Keogh J. Possible stimuli for strength and power adaptation: acute mechanical responses. Sports Med 2005;35:967-89.

23. Shimano T, Kramer WJ, Spiering BA, et al. Relationship between the number of repetitions and selected percentages of one repetition maximum in free weight exercises in trained and untrained men. J Strength Cond Res 2006;20:819-823.

24. Suter E, Herzog W, Sokolosky J, et al. Muscle fiber type distribution as estimated by Cybex testing and by muscle biopsy. Med Sci Sports Exerc 1993;25:363-70.

25. Babyak MA. What you see may not be what you get: a brief, nontechnical introduction to overfitting in regression-type models. Psychosom Med 2004;66:411-21.

26. Dat ML, McGuigan MR, Brice $G$, et al. Monitoring exercise intensity during resistance training using the session RPE scale. J Strength Cond Res 2004;18:353-58.

27. Gearhart RF, Goss FL, Lagally KM. Ratings of perceived exertion in active muscle during high-intensity and low-intensity resistance exercise. J Strength Cond Res 2002;16:87-91. 



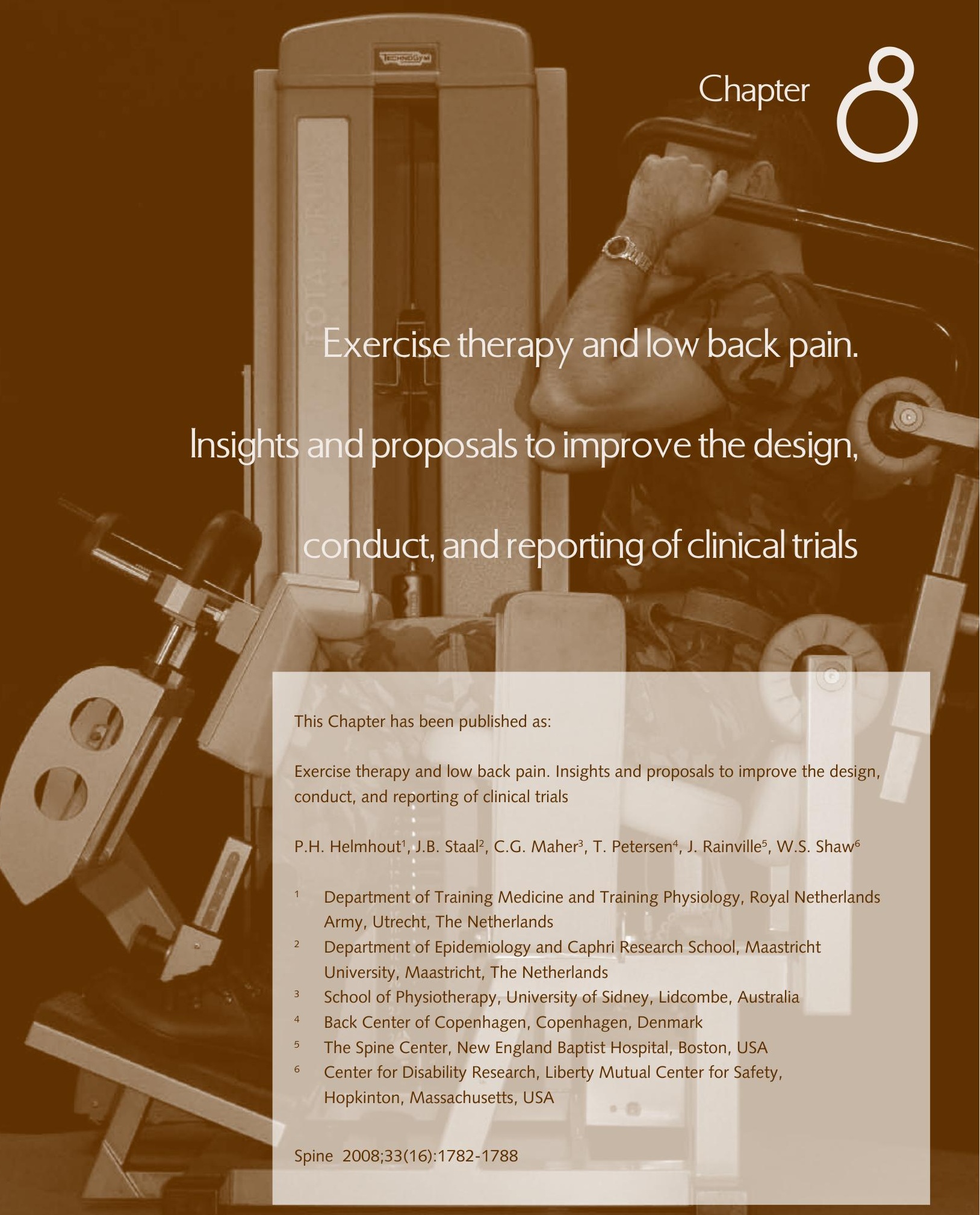




\section{Abstract}

Study Design: A report on plenary presentations and discussions of an expert panel and workshop ("Exercise as a treatment for sub-acute low back pain") that was held at the Amsterdam Forum VIII on Low Back Pain Research in Primary Care.

Objective: To highlight important issues regarding the design, conduct and reporting of exercise trials and to provide suggestions for improvement in each domain.

Summary of Background Data: Despite improvements in the methodological quality of randomized clinical trials on low back pain, current trials on exercise therapy still show methodological flaws in the design and reporting of studies. Methods: Important issues in the design, conduct, and reporting of exercise trials for low back pain were introduced by 4 presenters, and this was followed by a group discussion among 40 attendees. Conclusions and recommendations were extracted by the workshop panelists and summarized in this paper.

Results: Workshop discussion led to the following recommendations: (1) Specify a theoretical framework for exercise therapy for designing the intervention and selecting appropriate treatment efficacy measures; (2) To overcome blinding problems in exercise trials, include sham interventions and assess the success of blinding; (3) Evaluate the role of patient-provider interactions through the use of self-report or observational measures; (4) To assure quality of treatment, use exercise interventions that reference existing exercise guidelines; (5) Use subgroup analyses to identify subgroups of patients most likely to benefit; (6) In reporting exercise trials, include an extensive description of the study population, a detailed exercise protocol, and some measure of patient compliance; (7) Further categorization of exercise interventions is needed, in terms of concept, mode, intensity, duration, frequency, and length; and (8) Trial registration and publication of detailed study protocols may help to strengthen the evidence base concerning exercise therapy for low back pain. 
Conclusions: By paying proper attention to issues concerning the design and reporting of exercise protocols when conducting future exercise trials, research may provide a stronger evidence basis for recommending or not recommending various exercise therapies for low back pain.

\section{Introduction}

Hundreds of trials evaluating exercise therapy in low back pain (LBP) have been published in the last 50 years, the results of which have been summarized in several systematic reviews. Recent reviews conclude that exercise is an effective treatment for chronic LBP, $1 ; 2$ though improvements in outcomes such as pain and functional limitations are generally small and short-lived. Maintenance of exercise benefits may require patient education and motivational strategies to reinforce behavioural change and exercise compliance. ${ }^{3}$

While exercise is sometimes regarded as a single class of treatment, this conceptualization has failed to recognize the high variation among exercise programs and treatment protocols. The types of exercise programs for LBP vary widely from land-based exercise versus exercise in water to isolated trunk exercise versus a walking program, and it is unlikely that all programs are equally effective for all patients. Reviewers have routinely attempted a single summary conclusion on the efficacy of exercise, and this may contribute to its small reported effects.

The widespread publication of methodological guidelines ${ }^{4-7}$ in the last decade has led to an overall improvement in methodological quality of randomized clinical trials in the field of LBP research. ${ }^{8}$ Many trials on the effectiveness of exercise, however, still show methodological flaws in the design, conduct, and/ or reporting of the study. Internal validity criteria such as blinding, compliance, and co-intervention are often not met in exercise studies. ${ }^{2 ; 9}$ For example, in trials published since 2000 on exercise therapy for LBP, only $39 \%$ were assessor blinded, $42 \%$ used intention to treat analysis, and $40 \%$ concealed allocation. ${ }^{10}$ Low quality is assumed to be associated with biased estimates of treatment effects in both randomized and nonrandomized clinical trials, although the literature is 
not consistent on this topic ${ }^{11 ; 12}$, neither is it on the validity of different scales to measure trial quality ${ }^{12 ; 13}$. Furthermore, generalizability of the results of trials dealing with exercise might be violated by voluntary participation because back pain patients who have no affinity for exercise, or have fear-avoidance beliefs about physical activity, are supposedly less likely to participate. ${ }^{14 ; 15}$

The strict eligibility criteria used in many exercise trials (e.g., cardiovascular contraindications for participating in progressive exercises) might also select people with a higher capacity to benefit from exercise therapy, resulting in larger estimates of effects. Beside internal and external validity criteria of experimental designs, another issue is to what extent the treatment is applicable and relevant to various provider types and treatment settings. Criteria concerning applicability and clinical relevance allow providers from various disciplines to translate the results of exercise trials into guidelines for their clinical practice. For these reasons, researchers have expressed a need to improve the quality of reporting for exercise trials. ${ }^{16}$

The present paper highlights important issues regarding the design, conduct and reporting of exercise trials and provides suggestions for improvement in a number of domains. These analyses and recommendations are based on plenary presentations and discussions during and after an interactive workshop entitled "Exercise as a treatment for sub-acute low back pain" that was held at the Eighth International Forum on Low Back Pain, in Amsterdam, The Netherlands, in June 2006. Presentations of 4 panellists focused on: (a) mechanisms of action of exercise, (b) different aspects of treatment quality in exercise, (c) identifying subgroups among LBP patients, and (d) the publication of study designs. Subsequent discussion amongst the expert panellists included the topics of the plenary sessions, reactions to these presentations, as well as other topical areas (e.g., the role of patient-provider interaction within exercise protocols).

While it was beyond the scope of the symposium to address all methodological aspects of exercise studies for back pain, the expert panellists identified a number of key methodological issues that may represent threats to internal validity in 
the study of exercise treatment effects. The symposium also led to some specific suggestions; for example, using certain measurement instruments to improve the quality and reporting of results. The issues raised in the symposium may benefit future studies and facilitate the pooling of results in systematic reviews and meta-analyses.

\section{Designing and conducting exercise studies in low back pain}

\section{Mechanism of action of exercise}

Despite the considerable body of evidence for beneficial effects of exercise in LBP, there is a lack of knowledge about the mechanism of action of exercise. 17;18 The different theories to explain the mechanisms of action of exercise can be roughly grouped into (i) mechanical theories (e.g., spinal stabilization or core strengthening), (ii) neurological theories (e.g., desensitization), and (iii) cognitive and/or operant conditioning theories (e.g., graded activity). In these theories, exercise can be used either as a physical or behavioural tool to improve physical function, to reduce back pain intensity, and to reduce back pain-related disability. ${ }^{19}$

Why are theoretical frameworks important in exercise trials? Theory-based treatments specify a priori the most important active ingredients of the exercise intervention. The theory may be supported or not supported, but its specification provides an important basis for operationalizing intervention components. A conceptual framework gives direction to the actual content of the intervention, as well as the choice of a reference or "placebo" (nontreatment) intervention that maximizes the contrast on the characteristic underlying the presumed mechanism of action, and minimizes the contrast on other characteristics. A conceptual framework should also determine to a large extent the choice of a valid set of treatment process measures and prognostic variables.

As stated elsewhere, ${ }^{14 ; 17 ; 18}$ there is an urgent need for studies that examine whether improvements in those characteristics altered by exercise are, in fact, related to improvements in primary outcomes such as pain, functional status or return to work. If this is the case, it shows that the presumed mechanism of the 
intervention is more than just a label. For example, when using the frequently described "disuse" syndrome ${ }^{20}$ as the chosen mechanism of action in an exercise trial, a physical reconditioning approach is indicated in which quantification of physical capacity (e.g., measurements of muscle strength and lumbar spine mobility) is a key element. On the other hand, if operant conditioning principles for pain ${ }^{21}$ are chosen as the conceptual framework, instruments should be considered that measure, for example, changes in positive reinforcement of healthy behaviours and changes in withdrawal of attention towards pain behaviours.

\section{Blinding of patients and providers}

Blinding of patients and providers are the least fulfilled methodological criteria in exercise trials. ${ }^{8}$ This is to be expected because complete concealment is difficult to achieve because of mostly visible and/or tangible differences between real and placebo forms of exercises. Moreover, incidental conversations between patients, providers or assessors may lead to unblinding in many clinical settings. ${ }^{9}$ Blinding of patients may influence responses to treatment. For example, bias may be prominent when patients who are assigned to a new treatment in which innovative technology is used bring in favorable expectations, increased anxiety and higher treatment satisfaction compared to patients who are assigned to usual care treatment ("novelty bias"). ${ }^{22}$

What are the possibilities for blinding patients, providers and evaluators in exercise trials? First, use of independent staff and close control of the trial environment should allow for blinding of those responsible for outcome measurement, data entry and analysis. Secondly, it may be possible to blind the patient by using a sham intervention. Possibilities include sham versions of electrophysical treatments (e.g., sham ultrasound ${ }^{23}$, sham shortwave ${ }^{24}$ ), or sham versions of exercise where subjects exercise at a dose believed to be sub-therapeutic. 25;26 With some subterfuge and skill on the part of the therapist it may be possible to blind the patient so that they are unaware of whether they are in the real or sham exercise group. However, this blinding is not as robust as is possible in a placebo-controlled drug trial. Prior experience of exercise or the sham treatment, the therapist's manner, the title of the trial on the subject information sheet (e.g., a "placebo-controlled trial of exercise") or information on the trial 
obtained from a public trial registry may readily unblind the patient. Because patient blinding is so easily corrupted, it may be sensible to assess the success of blinding during the trial.

Although the item of "blinding" is enlisted in many scales that classify published RCTs according to their quality, the actual impact of this quality measure on treatment effects is still largely unknown. Few studies have examined the effects of blinding on quality assessment of clinical trials, and the results show little consistency in direction or magnitude..$^{11 ; 12}$

\section{Patient - provider interaction}

In exercise programs, there is always a strong supportive interaction between the provider of exercise (physician, physiotherapist, physical trainer, etc.) and the patient. Providers need to use their communication skills to successfully guide patients through a process of desensitization of fears and concerns, altering pain attitudes and beliefs, and/or improving affect, depending on the goal of the intervention. Patients beliefs in, and adherence to, the exercise is likely to be directly correlated to the professionalism that providers display, making this an essential part of the treatment delivered. Researchers should therefore attempt to balance personnel factors (e.g., courtesy, concern, and competency) among their treatment groups by means of appropriate training and monitoring. 27:28

Literature is scarce on the effect of patient-provider interaction on treatment success in LBP. Studies on this topic seem to reveal a gap between what is offered by health care providers and what is expected by patients (e.g., an explanation and legitimization of the pain, pain relief, and/or instruction and advice)..$^{29-31}$ For example, patients may hope that providers will legitimize their back pain by applying a somatic label such as 'slipped discs' or 'entrapped nerves'. The healthcare provider, on the other hand, may wish to avoid medical jargon and focus on global reassuring messages so that fear avoidant beliefs are not inadvertently reinforced. Patient confidence and expectations ${ }^{13 ; 29 ; 32 ; 33}$, the level of agreement between patient and provider on the chosen treatment strategy ${ }^{29: 33 ; 34}$, and patient satisfaction during and after treatment ${ }^{30 ; 34-36}$ are seen as strong and closely interrelated treatment effect modifiers. Although the theo- 
retical framework for integrating these factors into research designs is not well elaborated, they should be assessed in exercise trials nonetheless.

Clearly there is a need for tools to measure different aspects of the interaction between patient and provider. One example is the Patient-Practitioner Orientation Scale (PPOS), an 18-item self-report questionnaire that measures the roles that doctors and patients believe each should play in the course of their interaction. ${ }^{37 ; 38}$ Higher scores on the measure reflect a more patient-centered orientation. Improved outcomes are hypothesized when there is a higher level of concordance between patient and provider. Another, slightly more laborious method for quantifying the effect of patient-provider interaction is to audio-tape interactions between patients and providers during exercise sessions, develop a coding strategy for evaluating interactions, and then relate these to treatment outcomes. One such method that has undergone substantial testing and validation among physicians is the Roter Interaction Analysis System (RIAS), derived from social exchange theory that focuses on elements of interpersonal influence, problem solving, and reciprocity in the context of patient-physician encounters. ${ }^{39 ; 40}$ Such methods could be readily adapted to assess patient-provider interactions during exercise sessions.

\section{Treatment quality}

Herbert and $B \varnothing^{41}$ emphasize the importance of treatment quality in studies of complex health care interventions such as exercise therapy. These authors assert that some of the variability in estimates of effects between trials in meta-analyses can be explained by the variation in quality of administration of interventions in different settings. Below, we provide examples of what we believe are measures to improve treatment quality:

a. Use existing guidelines for exercise interventions ${ }^{3 ; 42}$, as well as certified and experienced providers of exercise. Published research can be used as a bench mark for defining an appropriate exercise program in a research context. ${ }^{41}$

b. Use clear and sufficiently detailed training protocols and/or guidelines, providing a reasonable amount of variation while also providing a specific algorithm for providers to follow for modifying the intervention based on individual characteristics in a reproducible fashion. Little is known thus far on the 
beneficial influence of provider adherence to clinical practice guidelines on (perceived) treatment outcomes and health costs in LBP, although a recent cross-sectional study within the US military healthcare service provided some evidence in this direction. ${ }^{43}$ The main benefit of clinical guidelines, however, is to improve the quality of care in terms of a more transparent, evidencebased clinical decision process. ${ }^{44 ; 45}$

c. Limit the number of providers involved in an exercise program, thereby redu cing inter-person variability in the administration of the treatment.

d. Organize a "feed-forward session" and several "feedback sessions" with pro viders in a pilot-phase of the trial, in which different aspects of the trial design can be explained, instructions on the training protocols can be given, treatment actions can be practiced, and individual patient cases can be discussed.

e. Plan a practice period in the pilot phase of the study, in which the treatment under study can be administered within the logistics of the upcoming trial. To monitor treatment quality during the study, one could organize for an experienced clinician (not involved with the research group) to audit participants from a randomly selected sample of the treatment sessions, in order to assess whether treatment is performed as intended. Other possibilities are to make (video) observations of treatment sessions for quality assessment by an independent expert clinician, or to plan regular "monitoring visits" to the treatment locations.

\section{Subgroup analyses}

It seems unlikely that any one exercise approach would be equally beneficial for all patients with LBP. ${ }^{46}$ However until recently, most reviews and guidelines ${ }^{1 ; 5 ; 47 ; 48 ; 49}$ concluded that based on the existing evidence no category of exercise appears to be better than others. Several authors have proposed that the modest treatment effects seen with exercise might at least partly be explained by the heterogeneity of the samples of nonspecific LBP patients included, i.e., patients are heterogeneous with regard to their specific needs and expectations. Thus, only a minority of patients benefit from any one particular intervention approach. Adequate classification of homogeneous subgroups of LBP patients could improve diagnosis and treatment efficacy. ${ }^{50-52}$ 
This brings us to the discussion of performing subgroup analyses in exercise studies. If patients could be subdivided into groups based on the nature of physical, psychological, and/or organizational barriers to recovery, matching them to appropriate interventions may improve outcomes and reduce overall costs. Several clinically relevant classification systems for the subgrouping of LBP patients have been proposed, comprising pathoanatomical oriented systems that focus on the identification of the structural pain souce, i.e intervertebral discs, sacroiliac joints, or nerve root problems), or treatment oriented systems that focus on the matching of particular patterns of signs and symptoms with specific types of exercise therapy. ${ }^{53}$

There is a need for studies testing the efficacy of a particular type of exercise therapy on patients selected by criteria ("clinical prediction rules") derived from observational studies in combination with clinical experience. If possible, criteria should be based on theory supporting the ability of a specific type of exercise therapy to influence certain biological or psychosocial characteristics. Within the pathoanatomical approach, for instance, there is preliminary evidence that a composite of certain clinical tests might be useful to identify the disc or sacroiliac joint as the source of low back pain. ${ }^{54}$ Within the treatment oriented approach, observational studies have succeeded in developing a clinical prediction rule from associations between certain physical examination findings and treatment success following specific mobilizing exercises or training of neuromuscular control. ${ }^{55: 56}$ The effectiveness of prediction rules for the identification of particular subgroups of patients with LBP who would benefit from a specific type of exercise should be compared with that of nonspecific exercises in randomized clinical trials. For example, two studies have reported that choice of treatment based on a treatment oriented classification approach resulted in improved disability as compared with treatment unmatched to their classification or treatment based on clinical practice guidelines..$^{5758}$ Long et al ${ }^{59}$ have shown that in patients with a directional preference to movement, exercises in the preferred direction provided better results than exercises in a random direction or opposite direction. Finally, a study by George et al ${ }^{60}$ suggested that patients with fear-avoidance beliefs, improve more with exercise treatment that addresses the fear- avoidance than with standard care. 
If no plausible theory exists, the performance of post hoc analyses for the purpose of hypothesis generating may be indicated, i.e., analyses for associations between baseline patient characteristics and outcomes of a specific type of exercise. When using multivariable regression models, one needs to be mindful of the known limitations (e.g., risk of overfitting) of such analyses. ${ }^{61-63}$

\section{Reporting exercise studies in low back pain}

\section{Description of study population, exercise protocol, and compliance}

As mentioned earlier, exercise trials may suffer from a lack of generalizability. It is therefore of utmost importance that researchers give an explicit description of their study population, in order to enable practitioners to relate study results to their own patient population. ${ }^{14}$ The revised CONSORT Statement ${ }^{4}$ emphasises the importance of describing the recruitment method, eligibility criteria used in the study, and treatment allocation procedure. Together with baseline characteristics of the study population, these items give an idea of the internal validity of the trial. Also, the timing of the treatment in relation to the duration of the episode of pain should be described, as Staal and colleagues ${ }^{31}$ suggest. This is necessary so that the effectiveness of the intervention can be judged within the context of the natural course of LBP.

Authors of trials should describe exercise interventions in sufficient detail so that other researchers and practitioners could replicate the treatment and reviewers can make sensible decisions about pooling similar exercise programs. Obviously, the same applies to the description of control and "placebo" exercise interventions, home-exercises, and, possibly, co-interventions. For example, when using progressive resistance exercise as a physical therapy intervention, a clear description of training principles according to the American College of Sports Medicine (ACSM) is warranted. ${ }^{64}$ Table 1 categorizes the characteristics of different exercise concepts that, ideally, should be mentioned in trial reports. Behavioural treatments that include physical exercise, for example an operant therapy using graded activity, can be quantified according to the same categorization. 
Table 1. Exercise quantification scheme. Developed from: Bertina FM. Training program quantification: a practical approach. Annales Medicinae Militaris Belgicae 1994;8:78-82.

\begin{tabular}{|c|c|c|c|c|c|}
\hline category/ concept & mode & intensity & frequency & duration & length \\
\hline aerobic & $\begin{array}{l}\text { whole-body, } \\
\text { lower-body, } \\
\text { upper-body, } \\
\text { etc. }\end{array}$ & $\begin{array}{l}\text { - active recovery } \\
\text { - extensive endurance } \\
\text { - intensive endurance } \\
\text { - extensive interval } \\
\text { - intensive interval }\end{array}$ & \multirow[t]{3}{*}{$\begin{array}{l}\text { times per week/ } \\
\text { months/ etc. } \\
\text { number of days } \\
\text { between sessions }\end{array}$} & \multirow[t]{8}{*}{$\begin{array}{l}\text { minutes, } \\
\text { hours, } \\
\text { etc. }\end{array}$} & \multirow[t]{8}{*}{$\begin{array}{l}\text { weeks, } \\
\text { months, } \\
\text { etc. }\end{array}$} \\
\hline muscular strength & $\begin{array}{l}\text { own bodyweight, } \\
\text { free weights, } \\
\text { training machines, } \\
\text { etc. }\end{array}$ & 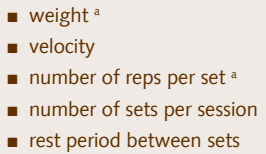 & & & \\
\hline muscular endurance & $\begin{array}{l}\text { idem muscular } \\
\text { strength }\end{array}$ & - idem muscular strength ${ }^{b}$ & & & \\
\hline $\begin{array}{l}\text { neuromuscular } \\
\text { control }\end{array}$ & $\begin{array}{l}\text { choice of system } \\
\text { (e.g., as described } \\
\text { by O'Sullivan, } \\
\text { Sahrman, McGill) }\end{array}$ & $\begin{array}{l}\text { criteria for progresion descri- } \\
\text { bed: number of reps per set }\end{array}$ & \multirow{5}{*}{$\begin{array}{l}\text { number of sessions } \\
\text { per day } \\
\text { times per week/ } \\
\text { months/ etc. } \\
\text { number of days } \\
\text { between sessions }\end{array}$} & & \\
\hline mobilizing & $\begin{array}{l}\text { choice of system } \\
\text { stated (e.g., Mc- } \\
\text { Kenzie-method) }\end{array}$ & $\begin{array}{l}\text { - number of reps per set } \\
\text { - number of sets per session }\end{array}$ & & & \\
\hline stretching & - & - & & & \\
\hline functional exercises & $\begin{array}{l}\text { exercises aiming } \\
\text { at physical activi- } \\
\text { ties in daily life }\end{array}$ & \multirow[t]{2}{*}{ - idem muscular strength } & & & \\
\hline $\begin{array}{l}\text { behavioural treat- } \\
\text { ment using exercise }\end{array}$ & $\begin{array}{l}\text { exercises aiming } \\
\text { at reducing fear } \\
\text { and/or altering } \\
\text { cognition }\end{array}$ & & & & \\
\hline
\end{tabular}

Notes: ${ }^{a}$ less than 15 to 20 reps (= repetitions) until failure, $70 \%$ of one-repetition maximum or more. b over 15 to 20 repetitions until exhaustion, less than $70 \%$ of one-repetition maximum.

One of the major sources of variation in the observed outcomes of clinical trials is the level of commitment and motivation among study participants for completing the full exercise program. ${ }^{41}$ When describing patient compliance, the following 4 items should ideally be addressed:

a. the way in which exercise characteristics were recorded, for example by keeping a diary of exercises or by filling out a training form;

b. the way in which supervision was delivered, i.e., fully or partly supervised programs versus nonsupervised programs (e.g., home-exercises); both in the ACSM guidelines for exercise prescription ${ }^{42}$ and in a recent review on exercise therapy ${ }^{65}$, the importance of supervision for exercise compliance is emphasized; 
c. whether intended or unintended co-interventions were at issue, exposing patients to other components than pure exercise, such as cognitivebehavioural approaches, ergonomic measures, or education;

d. whether adverse effects could be reported that were caused by the exercises.

\section{Trial registration and publication of study design}

Trial registration and protocol publication before study commencement have become more customary among studies of LBP. Recently, several journals jointly decided to only consider trials for publication if they have been registered before the enrolment of the first patient. ${ }^{66 ; 67}$ One of the mean reasons for this new policy is to prevent publication bias in scientific literature: it is known that trials with adverse or negative results are less likely to be submitted for publication. ${ }^{68}$ Researchers who register their trial and publish their study design beforehand will be more inclined to publish results. In any case, data can still be requested from the researcher for inclusion in a systematic review.

Amongst other advantages, ${ }^{69 ; 0}$ publication of a design article usually gives authors more space to elaborate on the specific content of the intervention. Systematic reviews on exercise therapy would benefit by the ability to distinguish among different types of exercise. Moreover, detailed information gives practitioners more insight into the practical application of the intervention, which contributes to an easier implementation in practice. Full-text, free-access articles from online journals, a trend in scientific literature, also helps in this matter. In the near future, access to treatment manuals and/or databases might be established not only by contacting the authors involved, but also by visiting a study website.

\section{Concluding remarks}

In summary, the design of exercise protocols requires attention to a number of important clinical and experimental factors that have not been adequately addressed or reported in many of the existing clinical trials. These include: (1) identifying a specific physiological and/or psychological mechanism of action; (2) selecting appropriate interventions and treatment efficacy (outcome) 
measures given the theoretical model and hypothesis under investigation; (3) evaluating the role of patient-provider interactions through the use of self-report or observational measures; (4) describing the intended role of patient-provider interaction to further treatment goals; (5) assuring quality of treatment by using exercise interventions that reference existing exercise guidelines or by assessing provider adherence through training or protocols; and (6) identifying subgroups of patients who are most likely to benefit by appropriate use of subgroup analyses. In addition, significant improvements could be made in the way that investigators assess adherence to treatment protocols and report study results. To improve the dissemination and exchange of knowledge in this field and to reduce the potential for publication bias, more details regarding study population and intervention design could be published in separate methodological (design) articles prior to the release of study results. Such improvements may lead to a stronger evidence basis for recommending or not recommending various exercise therapies for LBP.

\section{Key Points}

- A workshop on exercise therapy in (subacute) low back pain was held at the Eighth International Forum on Low Back Pain Research in Primary Care.

- The aim was to highlight important issues regarding the design, conduct and reporting of exercise trials and to provide suggestions for improvement in each domain.

- Based on the workshop, recommendations are made to at least partly over come typical methodological problems of exercise trials, such as lack of conceptual frameworks, blinding limitations, patient-provider interaction, and treatment quality.

- Moreover, significant improvements could be made in the way that investigators assess adherence to treatment protocols and report study results. 


\section{References}

1. van Tulder MW, Koes B, and Malmivaara A. Outcome of non-invasive treatment modalities on back pain: an evidence-based review. Spine 2006;15:S64-S81.

2. Hayden JA, van Tulder MW, Malmivaara AV et al. Meta-analysis: exercise therapy for nonspecific low back pain. AnnInternMed 2005;142:765-75.

3. Liddle SD, Baxter GD, and Gracey JH. Exercise and chronic low back pain: what works? Pain 2004;107:176-90.

4. Altman DG, Schulz KF, Moher D et al. The revised CONSORT statement for reporting randomized trials: explanation and elaboration. Ann Intern Med 2001;134:663-94.

5. Philadelphia Panel evidence-based clinical practice guidelines on selected rehabilitation interventions for low back pain. PhysTher 2001;81:1641-74.

6. van Tulder M, Furlan A, Bombardier C et al. Updated method guidelines for systematic reviews in the cochrane collaboration back review group. Spine 2003;28:1290-9.

7. Begg C, Cho M, Eastwood S et al. Improving the quality of reporting of randomized controlled trials. The CONSORT statement. JAMA 1996;276:637-9.

8. Koes BW, Malmivaara A, and van Tulder MW. Trend in methodological quality of randomised clinical trials in low back pain. Best Pract Res Clin Rheumatol 2005;19:529-39.

9. Philadelphia Panel evidence-based clinical practice guidelines on selected rehabilitation interventions: overview and methodology. PhysTher 2001;81:1629-40.

10. Maher CG. Quality of exercise trials for low back pain. Presentation held at workshop: Exercise as a treatment for sub-acute low back pain. Eight International Forum on Low Back Pain Research in Primary Care. 2006.

11. Moher D, Pham B, Jones A et al. Does quality of reports of randomised trials affect estimates of intervention efficacy reported in meta-analyses? Lancet 1998;352:609-13.

12. Balk EM, Bonis PA, Moskowitz $\mathrm{H}$ et al. Correlation of quality measures with estimates of treatment effect in meta-analyses of randomized controlled trials.JAMA 2002;287:2973-82.

13. Colle $F$, Rannou $F$, Revel $M$ et al. Impact of quality scales on levels of evidence inferred from a systematic review of exercise therapy and low back pain. Arch Phys Med Rehabil 2002;83:1745-52.

14. Moffett JK and Mannion AF. What is the value of physical therapies for back pain? Best Pract Res Clin Rheumatol 2005;19:623-38.

15. Vlaeyen JW and Crombez G. Fear of movement/(re)injury, avoidance and pain disability in chronic low back pain patients. Man Ther 1999;4:187-95.

16. Malmivaara A, Koes BW, Bouter LM et al. Applicability and clinical relevance of results in randomized controlled trials: the Cochrane review on exercise therapy for low back pain as an example. Spine 2006;31:1405-9.

17. Wessels $T$, van Tulder M, Sigl T et al. What predicts outcome in non-operative treat ments of chronic low back pain? A systematic review. Eur Spine J 2006;15:1633-44.

18. Staal JB, Hlobil H, van Tulder MW et al. Return-to-work interventions for low back pain: a descriptive review of contents and concepts of working mechanisms. Sports Med 2002;32:251-67.

19. Rainville J, Hartigan C, Martinez E et al. Exercise as a treatment for chronic low back pain. Spine J 2004;4:106-15. 
20. Verbunt JA, Seelen HA, Vlaeyen JW et al. Disuse and deconditioning in chronic low back pain: concepts and hypotheses on contributing mechanisms. Eur J Pain 2003;7:9-21.

21. Ciccone DS and Grzesiak RC. Cognitive dimensions of chronic pain. oc Sci Med 1984;19:1339-45.

22. Bouter LM, van Dongen MC. Epidemiologisch onderzoek: Opzet en interpretatie. Houten: Bohn Stafleu Van Loghum, 1995.

23. Faas A, Chavannes AW, van Eijk JT et al. A randomized, placebo-controlled trial of exercise therapy in patients with acute low back pain. Spine 1993;18:1388-95.

24. Tasclem R, Buth B, Koul $P$ et al. Chronic low back pain-comparitive analysis of treatment response to drugs and different physicial modalities. JK Pract 2003;10:201-4.

25. Manniche $C$, Hesselsoe $G$, Bentzen $L$ et al. Clinical trial of intensive muscle training for chronic low back pain. Lancet 1988;2:1473-6.

26. Helmhout PH, Harts CC, Staal JB et al. Comparison of a high-intensity and a low-in tensity lumbar extensor training program as minimal intervention treatment in low back pain: a randomized trial. Eur Spine J 2004;13:537-47.

27. Evans RL, Maiers MJ, and Bronfort G. What do patients think? Results of a mixed methods pilot study assessing sciatica patients' interpretations of satisfaction and improvement. J Manipulative Physiol Ther 2003;26:502-9.

28. Schers $H$, Wensing $M$, Huijsmans $Z$ et al. Implementation barriers for general practive guidelines on low back pain: A qualitative study. Spine 2001;26:E348-E353.

29. Azoulay L, Ehrmann-Feldman D, Truchon $M$ et al. Effects of patient-clinician disagreement in occupational low back pain: a pilot study. Disabil Rehabil 2005;27:817-23.

30. Verbeek J, Sengers MJ, Riemens $L$ et al. Patient expectations of treatment for back pain: a systematic review of qualitative and quantitative studies. Spine 2004;29:2309-18.

31. Staal JB, Rainville J, Fritz J et al. Physical exercise interventions to improve disability and return to work in low back pain: current insights and opportunities for improvement. J Occup Rehabil 2005;15:491-505.

32. Goldstein MS, Morgenstern H, Hurwitz EL et al. The impact of treatment confidence on pain and related disability among patients with low-back pain: results from the University of California, Los Angeles, low-back pain study.Spine J 2002;2:391-401.

33. Nordin M, Cedrschi C, and Skovron ML. Patient-health care provider relationship in patients with non-specific low back pain: a review of some problem situations. Baillieres Clin Rheumatol 1998;12:75-92.

34. Perreault K and Dionne CE. Does patient-physiotherapist agreement influence the outcome of low back pain? A prospective cohort study. BMC Muskuloskelet Disord 2006;7:76.

35. George SZ and Hirsh AT. Distinguishing patient satisfaction with treatment delivery from treatment effect: a preliminary investigation of patient satisfaction with symptoms after physical therapy treatment of low back pain. Arch Phys Med Rehabil 2005;86:1338-44.

36. Bombardier C. Outcome assessments in the evaluation of treatment of spinal disorders: summary and general recommendations. Spine 2000;25:3100-3.

37. Krupat E, Putnam SM, and Yeager CM. The fit between doctors and patients: can it be measured? J Gen Intern Med 1996;11:134. 
38. Krupat $\mathrm{E}$, Rosenkranz SL, Yeager $\mathrm{CM}$ et al. The practice orientations of physicians and patients: the effect of doctor-patient congruence on satisfaction. Patient Educ Couns 2000;39:49-59.

39. Sandvik $M$, Eide $H$, Lind $M$ et al. Analyzing medical dialogues: strength and weakness of Roter's interaction analysis system (RIAS). Patient Educ Couns 2002;46:235-41.

40. Roter D and Larson S. The Roter interaction analysis system (RIAS): utility and flexibility for analysis of medical interactions. Patient Educ Couns 2002;46:243-51.

41. Herbert RD and Bo K. Analysis of quality of interventions in systematic reviews. BMJ 2005;331:507-9.

42. American College of Sports Medicine. ACSM's guidelines for exercise testing and prescription. Philadelphia, PA: Lippincott Williams \& Wilkins, 2000.

43. Feuerstein $M$, Hartzell $M$, Rogers $H L$ et al. Evidence-based practice for acute low back pain in primary care: Patient outcomes and cost of fare. Pain 2006;124:140-49.

44. Bekkering GE, Hendriks HJM, van Tulder MW et al. Effect on the process of care of an active strategy to implement clinical guidelines on physiotherapy for low back pain: a cluster randomised controlled trial. Qual Saf Health Care 2007;14:107-12.

45. Woolf $\mathrm{SH}$, Grol R, Hutchinson A et al. Potential benefits, limitations, and harms of clinical guidelines. BMJ 1999;318:527-30.

46. Borkan JM, Koes B, Reis S et al. A report from the Second International Forum for Primary Care Research on Low Back Pain. Reexamining priorities. Spine 1998;23:1992-6.

47. Mior S. Exercise in the treatment of chronic pain. Clin J Pain 2001;17:S77-S85.

48. COST B13: European guidelines for the management of low back pain. Eur Spine J 2006;15 Suppl 2:s125-s300.

49. Slade SC and Keating JC. Trunk-strengthening exercises for chronic low back pain: a systematic review. J Manipulative Physiol Ther 2006;29:163-73.

50. Leboeuf-Yde C, Lauritsen JM, and Lauritzen T. Why has the search for causes of low back pain largely been nonconclusive? Spine 1997;22:877-81.

51. Bouter LM, van Tulder MW, and Koes BW. Methodologic issues in low back pain research in primary care. Spine 1998;23:2014-20.

52. Delitto A, Erhard RE, and Bowling RW. A treatment-based classification approach to low back syndrome: identifying and staging patients for conservative treatment. Phys Ther 1995;75:470-85.

53. McCarthy CJ, Arnall FA, Strimpakos N et al. The biopsychosocial classification of nonspecific low back pain: a systematic review. Phys Ther Rev 2004;9:17-30.

54. Hancock MJ, Maher CG, Latimer J, Spindler MF et al. Systematic review of tests to identify the disc, SIJ or facet joint as the source of low back pain. Eur Spine J 2007;16:1539-50.

55. Fritz JM, Whitman JM, and Childs JD. Lumbar spine segmental mobility assessment: an examination of validity for determining intervention strate-gies in patients with low back pain. Arch Phys Med Rehabil 2005;86:1753-62.

56. Hicks GE, Fritz JM, Delitto A et al. Preliminary development of a clinical prediction rule for determining which patients with low back pain will respond to a stabilization exercise program. Arch Phys Med Rehabil 2005;86:1753-62. 
57. Brennan GP, Fritz JM, Hunter SJ et al. Identifying subgroups of patients with acute/ subacute "nonspecific" low back pain. Spine 2006;31:623-31.

58. Fritz JM and George $S$. The use of a classification approach to identify subgroups of patients with acute low back pain. Spine 2000;25:106-14.

59. Long A, Donelson R, and Fung T. Does it matter which exercise? A randomized control trial of exercise for low back pain. Spine 2004;29:2593-2602.

60. George SZ, Fritz JM, Bialosky JE et al. The effect of fear-avoidance-based physical therapy intervention for patients with acute low back pain: results of a randomized clinical trial. Spine 2003;28:2551-60.

61. Babyak MA. What you see may not be what you get: A brief, nontechnical introduction to overfitting in regression-type models. Psychosom Med 2004;66:411-21.

62. Pocock SJ, Assmann SE, Enos LE, and Kasten LE. Subgroup analysis, covariate adjustment and baseline comparisons in clinical trial reporting: current practice and problems. Stat Med 2002;21:2917-30.

63. Brookes ST, Whitely E, Egger $M$, et al. Subgroup analyses in randomized trials: risks of subgroup-specific analyses; power and sample size for the interaction test. J Clin Epidemiol 2004;57:229-36.

64. Taylor NF, Dodd KJ, and Damiano DL. Progressive resistance exercise in physical therapy: a summary of systematic reviews. PhysTher 2005;85:1208-23.

65. Hayden JA, van Tulder MW, and Tomlinson G. Systematic review: strategies for using exercise therapy to improve outcomes in chronic low back pain. Ann Int Med 2005;293:2927-9.

66. Deangelis CD, Drazen JM, Frizelle FA, et al. Is this clinical trial fully registered? A statement from the International Committee of Medical Journal Editors. JAMA 2005; 293:2927-9.

67. Eysenbach G. Tackling publication bias and selective reporting in health informatics research: register your eHealth trials in the International eHealth Studies Registry. J Med Internet.Res 2004;6:e35.

68. Easterbrook PJ, Berlin JA, Gopalan R et al. Publication bias in clinical research Lancet 1991;337:867-72.

69. Dickersin K and Rennie D. Registering clinical trials. JAMA 2003;290:516-23.

70. Godlee F. Publishing study protocols: making them visible will improve registration, reporting and recruitment. BioMed Central News and Views 2001;2. 


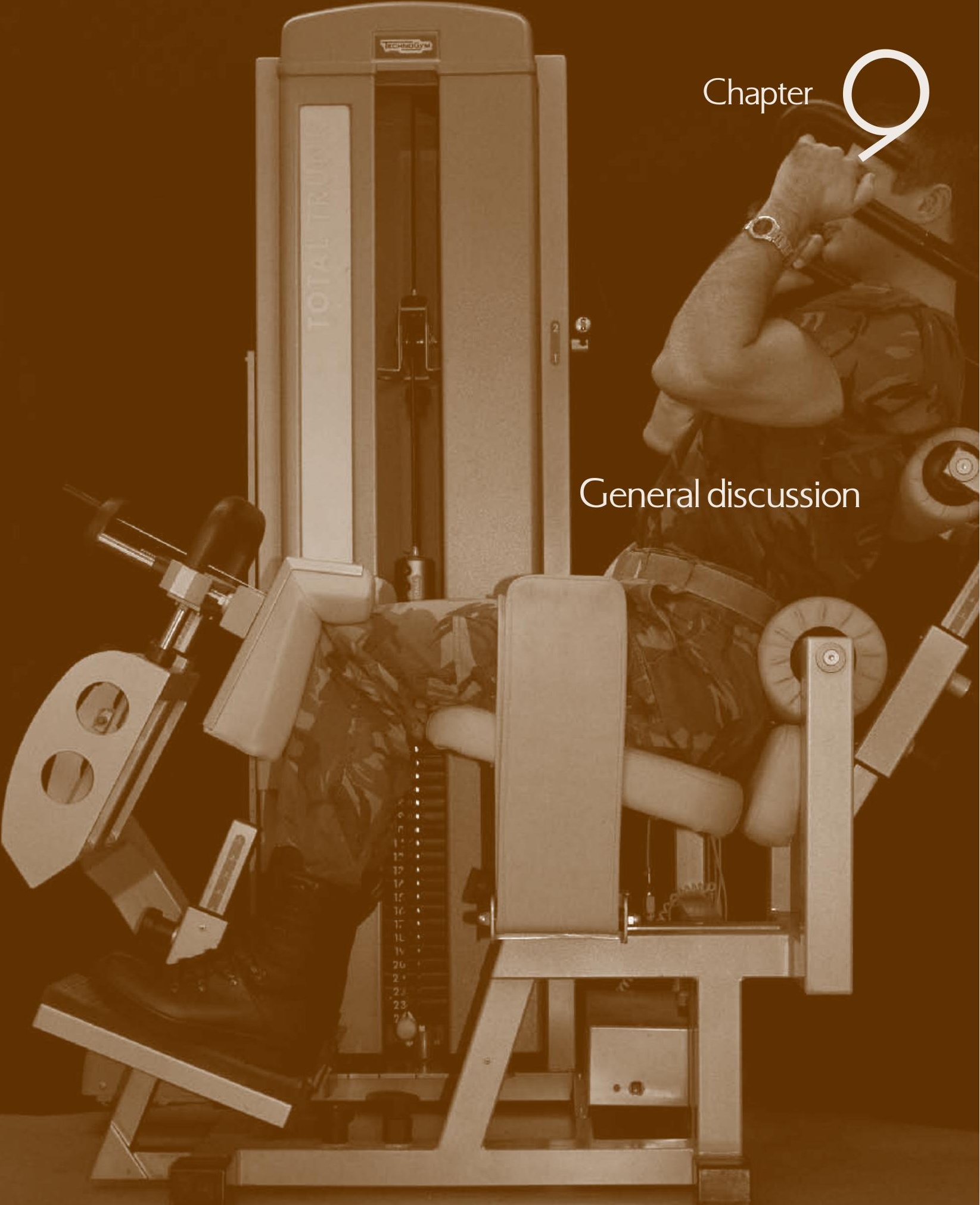


The main aim of this thesis was to evaluate the effectiveness of isolated Lumbar Extensor Strengthening (LES) in RNLA workers with 4 weeks or more of nonspecific LBP. Especially in the early nineties, several research groups have studied the efficacy of the LES concept in healthy subjects and low back pain (LBP) patients.1-8 Promising results were reported regarding strength improvements and pain relief in LBP patients, but methodological weaknesses (e.g. small population size, lack of randomization, inadequate or missing control groups) hinder solid interpretation of these findings. ${ }^{9-11}$ Besides, we postulated based upon our own experience with LES that several aspects of the concept would need revision, to generate a truly optimal stimulus for lumbar patients. Main principles of LES were the use of pelvic stabilization devices, a progressive resistance training approach, and the use of multiple isometric measurements. The Introduction Chapter of this thesis elaborates more on these LES principles, as well as on the way we modified the concept for our own research purposes.

In this chapter, the development of the LES concept and our research efforts on this topic are described first, to provide some insight in the methodological choices that we made when designing our trials. Next, a number of methodological issues regarding the randomized clinical trials that were performed in this thesis are discussed. The LES concept is positioned in the context of other scientific evidence in the field of LBP management, followed by a discussion on the implications for current (occupational) health care practice. Finally, some general recommendations are given for future research in the field of LBP management.

\section{How we developed and studied the LES concept}

Physical fitness is a critical aspect of military preparedness and an inherent part of military service. In modern society, high technology and mechanization have taken the place of hard physical labour and physical education has been de-emphasized. Military recruits that enter our organization may, therefore, suddenly meet physical demands far above those previously exposed to. One 
consequence of this shift to a more physically demanding life-style may be the occurrence of musculoskeletal injuries. Extensive information is available regarding the incidence rates of musculoskeletal injuries in military units of different nationalities. ${ }^{12}$ In most rating lists, LBP is ranked among the top three of training or work related musculoskeletal injuries.

For nearly three decades, the department of Training Medicine and Training Physiology of the Royal Netherlands Army (RNLA) has been involved in promoting functional strength and fitness amongst Dutch military recruits, both in preventive training and in rehabilitation programs for injured soldiers. At an annual meeting of the American College of Sports Medicine in the mid nineties, we were first introduced to the concept of isolated back training by a research group from the University of Florida's Center of Exercise, who were allied to the MedX Corporation and had developed the MedX Lumbar Extension Machine ${ }^{\circledR}$. For several reasons we were interested in this concept:

- the concept was based on sports medicine principles (e.g., resistance exercises, progressive loading, quota-based);

- the concept included highly time-efficient and protocollized treatment sessions of no more than 5 to 10 minutes on the machine, once or twice a week; we expected this 'minimal intervention strategy' to be appreciated in our military culture, in which longstanding and time-consuming rehabilitation is often not possible;

- the use a of training devices for fitness and rehabilitation purposes fitted with the training concepts of the RNLA, in which, from the very first military education, training equipment is used in the physical exercise programs of soldiers as well as in physiotherapy.

Some years before, the RNLA had selected state-of-the-art strength training machines from Technogym for their military fitness facilities. Since the MedX Lumbar Extension Machine was only available on the market as a rather expensive, compound test and training device (and not as a training device only), we used a Technogym Lower Back $\mathrm{ROM}^{\circledR}$ machine and modified it to such an extent that it could properly train the isolated lumbar extensors. Chapter 1 of this thesis provides some details about these modifications of the original LES 
concept. With the modified Technogym Lower Back ROM we performed several pre-studies, followed by two randomized controlled trials at our department in Utrecht. Initially, these in-house trials were meant to be 'pilot studies' for a future multicenter trial at RNLA military health centers. In the second half of 1998, all military (company) doctors and physiotherapists of the RNLA were requested to recruit participants with CLBP for our study. In addition, advertisements in military union media called for volunteers. Mainly due to this media attention, we received a lot of telephone calls and soon we had a list of over hundred-thirty volunteers. Due to the logistic limitations of our staff, we decided to start one trial with 88 participants in the spring of 1999. The other volunteers were placed on a waiting-list for a second trial, starting approximately one half year later. Data were collected from March 1999 through February 2000. The test and training protocol we used in both trials was partly based on existing protocols, ${ }^{2,6,8}$ and partly on our own clinical experience with LBP patients who had visited our department in the last few years. Important elements of these protocols were: a progressive resistance training stimulus at 50-70\% 1RM (15-20 repetitions) 1 or 2 times a week; an isometric back strength measurement every 4 weeks; special attention to the execution of the training in terms of pace and movement (full range of motion, slow and controlled movements while hollowing and flattening the lumbar lordosis). An extensive description of the design of both in-house trials can be found in Chapters 3 and 4 .

In 2001 we started working on the design of a multicenter trial. Physiotherapists of three RNLA health centres in The Netherlands (Amersfoort, Schaarsbergen, Oirschot) and one center in Germany (Seedorf) were asked to participate in this trial. Patient recruitment commenced in April 2002 and continued until December 2005. The reason for this long recruitment period was the relatively slow rate in which patients were enrolled in the study, which in its turn was influenced by our strict inclusion and exclusion criteria. For example, many military workers with LBP were not 'available during duty time for 10 consecutive weeks' due to varying military missions abroad and exercise periods outside the military base. Since the majority of military workers who participated in this trial had only moderate back complaints, few of them were fully absent from work. Moreover, the majority of soldiers with back complaints who visit the military 
health center have (semi-)acute LBP, which was an exclusion criterion for our trial. We attempted to increase the recruitment rate by including two more military health centers in our study (Den Haag in November 2003; Havelte in November 2004). This was only partly successful, since these additional locations together provided only 11 of the 127 (9\%) subjects that were included. An extensive description of the study, which we labelled as the Army Low BAck TRaining Study (short: ALBATRoS), can be found in Chapter 4.

\section{Interpretation of main study findings}

In the three trials of this thesis, short- and long-term effectiveness of our LES treatment concept was compared to a low-intensity variant of LES $\left(1^{\text {st }}\right.$ and $2^{\text {nd }}$ trial), to a non-treatment group ( $2^{\text {nd }}$ trial), and to usual RNLA care that mainly consisted of exercise therapy ( $3^{\text {rd }}$ trial), respectively. Effectiveness was primarily measured in terms of improvement in self-assessed functional disability. Overall, LES did not show any favourable outcomes over the other treatment programs.

These findings are in line with the current evidence regarding LBP management. Although it seems unlikely that any one exercise approach would be equally effective for all nonspecific LBP patients, most reviews and guidelines have concluded, thus far, that no category of exercise appears to be better than others. This may at least partly be explained by the heterogeneity of the included samples of nonspecific LBP patients with regard to their specific needs and expectations. Thus, only a minority of patients benefit from any one particular intervention approach. Adequate classification of more homogeneous subgroups of LBP patients could improve diagnosis and treatment efficacy. Developing classification systems and clinical prediction rules that validly identify nonspecific LBP subgroups are the real challenges for future research in this area. As far as our study population is concerned, one could reasonably say that in certain aspects this population can be considered rather homogeneous. The next paragraph on methodological considerations (sub-paragraph Patients and providers) will address this issue in more detail.

Another reason for the lack of favourable outcomes of LES over comparators could be the relatively low work load of our training regimes compared to those 
recommended in guidelines on training loads. We pursue this matter further in the paragraph on LES in the context of current LBP management (sub-paragraph Reconditioning of LBP patients using specific low back strengthening). Finally, methodological choices in our trial designs may to some extent have influenced the results. Some of these choices will be addressed in the next paragraph.

\section{Methodological considerations}

Methodological limitations as well as strengths of the different studies we conducted were already discussed in the earlier chapters. This paragraph will, therefore, focus more specifically on an overall discussion of a number of major methodological issues concerning the three randomized clinical trials of this thesis.

\section{Patients and providers}

The study group of, in total, 273 subjects that participated in our trials were predominantly male military workers from the RNLA. The recruitment procedure was partly different between the in-house trials (referrals by company doctor/ general practitioner as well as by advertisement) and the multicenter trial (referral by general practitioner). Moreover, the in-house studies included chronic LBP patients (> 12 weeks of continuous or recurrent pain) only, while the multicenter trial also included patients with sub-acute complaints (6-12 weeks). Despite these differences in study design, baseline characteristics of the three trial groups were mostly comparable. Baseline age, percentage of physically active workers, back strength, RMDQ score, and TSK score were similar between the groups; the percentages of workers with pain radiation and work absenteeism were slightly higher in the second in-house trial compared to the other studies. Because of the different cut-off points used in the inclusion criterion 'duration of complaints', the most prominent difference was, expectedly, the percentage of subjects with chronic LBP, being two times higher in the in-house trials than in the multicenter trial. Despite differences in study group characteristics, our main study results outcomes were consistent among the three trials.

At the start of the multicenter trial, nine of the 14 physiotherapists involved scored the Pain Attitudes and Beliefs Scale for Physiotherapists (PBAS-PT). This 
questionnaire can discriminate between physiotherapists with a 'behavioural' versus those with a 'biomedical' treatment orientation.13 All but one physiotherapist scored higher on the 'behavioural scale', with a mean standardized score of $49 \%$ [range $40-63 \%$ ] versus 33\% [range 11-49\%] on the 'biomedical scale'. This finding may reflect a slightly more behavioural-oriented attitude in our provider group towards LBP management. A behavioural-oriented attitude may conflict to some extent with the mainly biomedical character of LES, but we do not expect this to have had much influence on the study outcomes. The LES treatment program is highly protocolized, thus leaving little room for inter-individual variation in execution. Besides, all but one physiotherapist (who mainly provided ostheopathic treatment during usual care - not in the trial) already used exercise therapy with training devices in their treatment of LBP.

\section{Outcome measures}

The outcome measures used in our trials were mostly self-reported measures of functional disability (all trials), general health (in-house studies), and workrelated aspects (multicenter trial). We used functional disability, whether or not combined with self-perceived effect, as our primary outcome measure in all three studies. We considered 'functional restoration' as more relevant for our mainly chronically injured, working population than indicators of the symptom itself, such as pain intensity. The treatments in our trials were predominantly aimed at restoring physical functioning, not at reducing pain. We did not include a return-to-work measure in our analyses, since only a small subset of our study population was not working at the time of inclusion.

The only measure that was not self-reported, individual isometric back strength, was assessed with the measurement module of the lumbar training device. Because inconclusive evidence has been found in the literature for a relationship between lumbar muscle strength and LBP symptoms, ${ }^{14}$ we only used this measure to evaluate the effectiveness of our progressive LES program in terms of strength progression. The fact that we also used our lumbar training device for measurements of back strength may to some extent have led to an overestimation of the strength progression found in our trials (test effect). 


\section{Methodological quality}

To assess the overall quality of our trials, a criteria list for 'the believability and the interpretability of trial quality' can be used that has already been applied on our trials: the Physiotherapy Evidence Database (PEDro) Scale. ${ }^{15}$ This scale is based on the list developed by Verhagen et $\mathrm{a}^{16}$ using the Delphi technique. The reliability of the overall score on the scale has been qualified as 'fair' to 'good'. ${ }^{17}$ According to the PEDro scale, and using a proposed methodological score of 7 points or more on the 10-point scale as the criterion for "high quality", ${ }^{18}$ both in-house trials have been qualified as high-quality trials by trained raters allied to the Centre for Evidence-Based Physiotherapy in Sydney (http://www.pedro.org. au/index.html). See Table 1.

Table 1. Criteria list for the methodological quality assessment ${ }^{15}$.

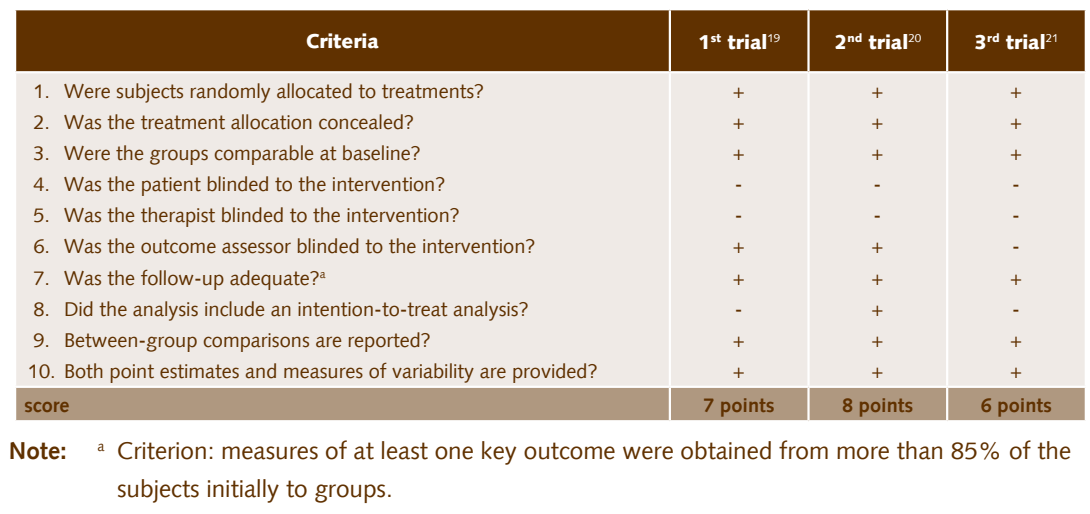

All three trials score expectedly low on items concerning blinding. In trials on exercise therapy, it is virtually impossible to blind patients and providers for the treatment allocation. At best, trials are performed as single-blinded trials in which treatment providers and outcome assessors are blinded for the treatment allocation. Provider blinding was not possible: our treatment approach was based on a one-to-one patient-provider interaction with the use of equipment and protocols that, for obvious reasons, could not be masked in any way. Therefore, blinding of the outcome assessor was the only measure we could take in our in-house trials: provision of training and data collection were conducted by different persons. To some extent, this was not possible in the multicenter trial due to limitations in the number of available physiotherapists at each study location. Participants filled in their questionnaires on a stand-alone personal compu- 
ter that was only accessible to an independent data manager. Strength measurements, however, needed to be administered by the providers of treatment. This may have led to the negative rating on item 8 of the PEDro Scale for this trial. One of the risks of failure to blind care providers is that an unequal provision of care according to the treatment administered. This so-called performance bias may have influenced the results to a certain extent, since our providers were always aware of the treatment program. However, the LES regimes used in our studies were highly protocollized, leaving little room for personal interpretation. The rate of both short- and long-term follow-up was comparable between the treatment programs in all trials, and co-intervention or contamination hardly occurred, making performance bias less likely.

We believe to have used state-of-the-art statistical techniques to evaluate the outcomes of the studies in this thesis. In a longitudinal design with repeated measurements, as was the case in our trials, multiple linear regression analysis as well as random coefficient analysis can be considered as robust statistical techniques, with the latter having the benefit of including both complete and incomplete data. In the secondary analyses of the multicenter trial on prognostic LBP factors, we used a modern imputation technique (MICE) to account for the missing data that were mainly caused by merging the three trial databases. ${ }^{22}$

In general, we were able to perform our trials closely following the revised CONSORT Statement from 2001. ${ }^{23}$ However, several items that are emphasized in the 2008 extended version of the CONSORT Statement for randomized trials of non-pharmacologic treatment may not have been explicitly or adequately addressed in the reporting of our trials. ${ }^{24}$ First, eligibility criteria for centers and providers are recommended in the extended CONSORT Statement ("Item 3"), due to a possible relationship between patient outcome on the one hand and centers' volume, care providers' volume and expertise on the other hand. In a design article of our multicenter trial, we have reported on the eligibility criteria for health centers to be included in this trial: (1) representing a major part of the overall military RNLA population; (2) holding at least two full-time physiotherapists; and (3) the willingness to unconditionally participate in the project. ${ }^{25}$ The providers of treatment in our trials, 14 in total, consisted of 11 male and 3 
female physiotherapists with at least 5 years of experience in our organization. Treatment location was included in the longitudinal regression analysis of our multicenter trial, in an attempt to account for the variability that this variable might cause in our data.

Second, for a full appraisal of the applicability of the trial's results, authors should provide details of whether and how the clustering of patients by centers was addressed ("Item 13"). In our multicenter trial, three centers provided the large majority ( $81 \%$ ) of the participants. See Table 2.

Table 2. Allocation of patients per center in the multicenter trial ALBATRoS.

\begin{tabular}{|l|c|c|c|}
\hline Center & Total & Back Strengthening & Usual Care \\
\hline Schaarsbergen & 37 & 20 & 17 \\
\hline Amersfoort & 33 & 17 & 16 \\
Oirschot & 33 & 19 & 14 \\
Seedorf & 13 & 8 & 5 \\
Havelte & 6 & 3 & 3 \\
\hline Den Haag & 5 & 4 & 1 \\
\hline
\end{tabular}

Third, the use of 'placebo' interventions in trials should be debated ("Item 20"), since these treatments may have specific therapeutic effects or ethical aspects. This may particularly apply to our two in-house trials, in which a low-intensity training program was used as the comparator for our LES concept. We attempted to use a training load below what we thought was the physiological threshold of strengthening muscles, but still with some credibility towards participants. It appeared that, for individuals in the low-intensity program with extreme low baseline strength of the lumbar muscles, this threshold may not have been adequate, providing too large a training stimulus. This may have caused a lack of contrast between the two intervention programs in these trials.

The recommendations we enumerated in Chapter 8 on the design, conduct, and reporting of clinical trials have to some extent been adapted in our research efforts. We think we have succeeded in implementing the following items in the study design of the three trials:

- choosing exercise interventions, treatment process measures, and prognos- 
tic variables, respectively, based on a conceptual framework (i.e., physical de conditioning of the lumbar spine);

- using independent staff (providers versus evaluators);

- balancing personnel factors (e.g., work experience, treatment-orientation), training and monitoring (e.g., feed forward sessions, practice periods, center visits);

- patient satisfaction during and after treatment was assessed in the trial;

- using detailed training protocols for lumbar strengthening based both on guidelines and earlier research;

- performing secondary analyses by following guidelines to prevent potential pitfalls such as overfitting (e.g., using limited potential prognostic factors);

- giving explicit descriptions of the study populations and exercises in our publications;

- reporting on items such as exercise records, supervision, co-intervention, and adverse cases;

- registering our multicenter trial and publishing its study design on forehand.

Future research on the LES concept could emphasize methodological issues that we have scarcely/not addressed in our trials:

- retrospectively assessing the success of blinding;

- assessing patient beliefs and expectations regarding the treatment;

- using tools that measure different aspects of the interaction between patient and provider;

- using a theory-based classification system for the subgrouping of nonspecific LBP patients.

\section{Lumbar extensor strengthening in the context of current LBP management}

When we started our research activities on the lumbar back in the late nineties, health care adherence to the biopsychosocial model of LBP management had already started to overshadow the traditional biomedical view. For reasons already mentioned earlier, we believed at that time that the biomedical-orientated LES concept could still be of value for our military population. The next sections give an overview of our current view on LBP management, which is endorsed by both the findings in our studies and the scientific literature in this last decade. 
Physical deconditioning in LBP patients

Physical disuse, defined as decreased physical activity behaviour in daily life, may lead to cardiovascular and muscular deconditioning. This process has been presented as one of the dominant factors for chronicity in conceptual frameworks on pain. ${ }^{26}$ Already in the late eighties, the term "deconditioning syndrome" was introduced for chronic LBP patients who suffered from pain-induced behavioural and psychological problems and subsequent physical disuse, leading to physiological deconditioning and a further loss of function. ${ }^{27}$ Therefore, physical reconditioning has been proposed in clinical practice as a goal in the management of chronic pain, despite the lack of evidence that the (apparent) deconditioning problem in chronic pain patients exceeds its presence in the general population.

In two different reviews, Verbunt et $\mathrm{a}^{28}$ and Smeets et a ${ }^{29}$ have attempted to shed light on this issue. They both conclude that the presence of physical deconditioning in chronic LBP is not confirmed by the literature presented. Studies on cardiovascular deconditioning (mostly cross-sectional) are inconclusive about potential differences between the cardiovascular capacity of chronic LBP patients and healthy controls. Studies on muscular deconditioning show that chronic LBP patients may have disuse symptoms such as paraspinal muscle fiber atrophy and fatty infiltration. However, no consistent differences are found in studies comparing LBP patients with healthy controls matched for age and anthropometry. Research on muscle strength in chronic LBP that focuses on other muscle groups than the lumbar muscles, such as trunk and lower legs, imply that muscle weakness in chronic LBP patients is not just a local problem of the lower back, but a generalised problem that is probably facilitated by lower levels of physical activity. However, revealing behaviour of decreased physical activity in chronic LBP patients is methodologically challenging. Researchers are confronted with a lack of valid assessments for physical activity in LBP patients, but also with the confounding influences of motivational, cognitive, and work-status related factors. There is still a long way to go, but if future research shows that physical disuse leading to general deconditioning is more present in LBP patients compared to healthy subjects, and that this deconditioning plays a role in the complex of chronic LBP and its resulting disability, then it would make sense to focus LBP treatment on general fitness more than on lumbar extensor strengthening or 
other specific back training. The next paragraph gives an overview of the current evidence on exercise treatments in LBP in general, and on specific low back strengthening in particular.

\section{Reconditioning of LBP patients using specific low back strengthening}

An extended meta-analysis by Hayden et al30 in 2005 summarizes the evidence on the effectiveness of exercise therapy in nonspecific LBP thus far. They conclude that exercise:

- is not more effective than other conservative treatments or no treatment in acute LBP;

- may be effective in workers with sub-acute LBP when embedded in a graded-activity program;

- is at least as effective as other conservative treatments in chronic LBP. In a recent systematic review that also includes our first in-house trial, Smeets et $\mathrm{a}^{29}$ conclude that there is no strong evidence supporting the use of intensive exercise instead of other active therapies in the treatment of chronic LBP and its resulting disability. They report that only back extensor strengthening shows some proof for being more effective when provided 'with sufficient intensity and frequency to fulfil the exercise physiology criteria'. With 'sufficient', they refer to a threshold of 16 hours of training that has been proposed by Brontfort et al. ${ }^{31}$ In a systematic review by Hayden et al, ${ }^{32}$ a 'training intensity' ${ }^{\# 1}$ defined as 20 or more hours of intervention time has been identified as one of the critical success factors in LBP management. Other critical factors for effective application of exercise therapy in LBP were: individually designed program; individual supervision; stretching and/or muscle-strengthening exercises; additional other conservative treatment (e.g., NSAIDs, manual therapy).

Our LES training concept fulfils most of these criteria, but not the criterion of 'sufficient intensity'. The work load of our training regimes was: 1 or 2 times a

\footnotetext{
\#1 The term 'training intensity' is confusingly used in the literature to indicate both the work load of a training program (i.e., frequency and duration of training sessions in a certain period) and the training load used in an individual training sessions (often expressed in percentage of the one-repetition maximum). We have used 'intensity' throughout this thesis following the latter of the two definitions, for example in the 'high-intensity' versus 'low-intensity' treatment programs.
} 
week, one set of, on average, 10 minutes of training on the LES training device. This resulted in 14 training/test sessions in 12 weeks for the $1^{\text {st }}$ in-house trial, 10 sessions in 8 weeks for the $2^{\text {nd }}$ in-house trial, and 17 sessions in 10 weeks for the multicenter trial. Beside muscle isolation, another principle of LES is the relatively low number of training sessions that is prescribed for optimal results. Previous studies on the original LES concept reported improvements in strengths after training at a frequency of two or even one day per week comparable to improvements after a training frequency of three times per week. ${ }^{3,5,6}$ On the other hand, guidelines of the American College of Sports Medicine (ACSM) on progressive resistance training recommend training loads corresponding to an 8- to 12-repetition maximum (RM) be lifted in 1 to 3 sets, training 2 to 3 days each week for physiological training effects. ${ }^{33}$ Neither of our LES training regimes fulfill the ACSM criteria. All study groups, however, showed significant and sometimes clinically relevant changes over time. We cannot be sure that these changes reflect real treatment effects or merely a natural history of recovery, since only the $2^{\text {nd }}$ trial included a non-treatment group. But the fact that the majority of our study population had already longstanding complaints before entering the program (65\% had more than 1 year of back complaints) indicates that the post-treatment improvements found in our trials cannot always be explained by spontaneous recovery.

A more fundamental explanation for the lack of treatment specificity of various exercise modalities is given by Smeets et al. ${ }^{31}$ They suggest that, irrespective of the type of exercise, reactivation may be the common factor in all types of active treatments to reduce chronic LBP disability, possibly intermediated by psychosocial processes like fear-avoidance, self-efficacy or pain perception. One of these potential intermediating factors, fear of movement, was assessed in our studies with the Tampa Scale of Kinesiophobia (TSK). The primary 'quest' in our research project was to evaluate whether lumbar extensor strengthening had beneficial effects for subjects with nonacute nonspecific LBP that would, at all, exceed other (common) treatments in LBP management. In secondary analyses of a merged dataset of the three trials, nevertheless, we explored the potential importance of this and other patient factors associated with clinically relevant 
improvements in LBP. These analyses indicated a prognostic value of the TSK score for long-term improvement in LBP, irrespective of the type of treatment in the last 6 months. This finding may give some ground for the reactivation-hypothesis posed by Smeets et al, but further research in this area is warranted.

\section{LES in the current military health care practice}

In March 2008, we organized a plenary close-off meeting for the ALBATRoS project in Utrecht, which was attended by 8 of the 10 physiotherapists involved. The results of the study, as well as the experiences of the care providers with LES during and after the trial, were discussed. Without exception, care providers indicated to use the same proportion of time for different treatment modalities currently as they did during the ALBATRoS project. Five out of eight physiotherapists who still had a lower back test/training device at their health center at the time of the close-off used this device for rehabilitation purposes, three of them on a weekly basis and the other two incidentally. Half the group of physiotherapist who attended the close-off felt that LES had additional value for LBP management in the RNLA, the other half did not. Generally, opinions based on experiences with LES in research and practice appeared to be rather divergent amongst the physiotherapists involved, with approximately half of them embracing the concept and the other half rejecting it.

\section{Recommendations for future research}

Based on the findings from our research project, a number of new topics can be listed as well as methodological issues that need attention in future studies in this research area.

1. The findings of our studies on lumbar extensor strengthening corroborate the current evidence on exercise therapy for nonspecific LBP, which tells us that less intensive physical training programs (in terms of duration and frequency) show no additional effectiveness compared to other active treatments such as general exercise therapy. A new randomized trial using a LES intervention program of higher frequency and duration is required to evaluate whether LES has potential surplus value over other intensive physical training programs in the management of LBP. 
2. The secondary analyses of our trials indicate that fear of movement/(re)injury may have an important intermediating influence on LES-induced re conditioning in our study group. More longitudinal research is needed to further unravel this influence and to judge the value of treatment interventi ons specifically aimed at reducing fear avoidance behaviour in combination with lumbar strengthening.

3. We have used LES in our military study population, based on the idea that this basically biomedical-oriented concept might match the attitudes and beliefs of a typical male no-nonsense study group like ours. This hypothesis needs to be confirmed in prognostic research, which can also identify whether subgroups of nonspecific LBP patients benefit more from this ap proach than others. Subsequently, RCT's need to be performed that quantify the therapeutic effects achieved for patients treated with the protocols tailored to this sub-grouping, compared with the therapeutic effects in matched patients achieved with generic treatment protocols.

4. Chapter 8 of this thesis has further delineated important (methodological) is sues to improve the design, conduct, and reporting of clinical trials on exer cise therapy for LBP in general. Recommendations include: identifying a theoretical framework and choosing appropriate interventions and treatment efficacy (outcome) measures given this framework; considering the use of sham-interventions or at least assess the success of other blinding measures; evaluating the role of patient-provider interactions; describing the intended role of patient-provider interaction to further treatment goals; identifying subgroups of patients who are most likely to benefit by appropriate use of subgroup analyses; further categorizing exercise interventions in terms of concept, mode, intensity, duration, frequency, and length; providing more details regarding study population and intervention design by reporting separate methodological (design) articles prior to the release of study results. Such improvements may lead to a stronger evidence basis for recommending or not recommending various exercise therapies for LBP. 


\section{References}

1. Leggett S, Mooney V, Matheson LN, et al. Restorative exercise for clinical low back pain. A prospective two-center study with 1-year follow-up. Spine 1999;24:889-98.

2. Graves JE, Webb DC, Pollock ML, et al. Pelvic stabilization during resistance training: its effect on the development of lumbar extension strength. Arch Phys Med Rehabil 1994;75:210-5.

3. Tucci JT, Carpenter DM, Pollock ML, et al. Effect of reduced frequency of training and detraining on lumbar extension strength. Spine 1992;17:1497-501.

4. Graves JE, Pollock ML, Leggett SH, et al. Limited range-of-motion lumbar extension strength training. Med Sci Sports Exerc 1992;24:128-33.

5. Carpenter DM, Graves JE, Pollock ML, et al. Effect of 12 and 20 weeks of resistance training on lumbar extension torque production. Phys Ther 1991;71:580-8.

6. Graves JE, Pollock ML, Foster D, et al. Effect of training frequency and specificity on isometric lumbar extension strength. Spine 1990;15:504-9.

7. Graves JE, Pollock $M L$, Jones $A E$, et al. Specificity of limited range of motion variable resistance training. Med Sci Sports Exerc 1989;21:84-9.

8. Pollock ML, Leggett $\mathrm{SH}$, Graves JE, et al. Effect of resistance training on lumbar extension strength. Am J Sports Med 1989;17:624-9.

9. Taimela $S$, Diederich $C$, Hubsch $M$, et al. The role of physical exercise and inactivity in pain recurrence and absenteeism from work after active outpatient rehabilitation for recurrent or chronic low back pain: a follow-up study. Spine 2000;25:1809-16.

10. Nelson BW, O'Reilly E, Miller $M$, et al. The clinical effects of intensive, specific exercise on chronic low back pain: a controlled study of 895 consecutive patients with 1-year follow up. Orthopedics 1995;18:971-81.

11. Risch SV, Norvell NK, Pollock ML, et al. Lumbar strengthening in chronic low back pain patients. Physiologic and psychological benefits. Spine 1993;18:232-8.

12. Vogel JA, Vanggaard L, Hentze-Eriksen T. Injuries related to physical training. Ann Med Milit Belg 1994;83:49-56.

13. Ostelo RW, Stomp-van den Berg SG, Vlaeyen JW, et al. Health care provider's attitudes and beliefs towards chronic low back pain: the development of a questionnaire. Man Ther 2003;8:214-22.

14. Hamberg-van Reenen HH, Ariens GA, Blatter BM, et al. A systematic review of the relation between physical capacity and future low back and neck/shoulder pain. Pain 2007; 130:93-107.

15. Sherrington C, Herbert RD, Maher CG, et al. PEDro: a database pf randomised trials and systematic reviews in physiotherapy. Man Ther 2000;5:223-6.

16. Verhagen AP, de Vet HCW de Bie RA, et al. The Delphi List: a criteria list for quality assessment of randomized clinical trials for conducting systematic reviews developed by Delphi consensus. J Clin Epidemiol 1998;51:1235-41.

17. Maher CG, Sherrington C, Herbert RD et al. Reliability of the PEDro scale for rating quality of randomized controlled trials. Phys Ther 2003;83:713-21.

18. Davies RA, Maher CG, Hancock MJ. A systematic review of paracetamol for non-specific low back pain. Eur Spine J 2008;17:1423-30.

19. Harts CC, Helmhout PH, de Bie RA, et al. A high-intensity lumbar extensor strengthening program is little better than a low-intensity program or a waiting list cotrol group 
for chronic low back pain: a randomised clinical trial. Aust J Physiother 2008;54(1):23-31.

20. Helmhout PH, Harts CC, Staal JB, Candel MJ, de Bie RA. Comparison of a high intensity and a low-intensity lumbar extensor training program as minimal intervention treatment in low back pain: a randomized trial. Eur Spine J 2004;13(6):537-47.

21. Helmhout PH, Harts CC, Viechtbauer W, et al. Isolated Lumbar Extensor Strengthening Versus Regular Physical Therapy in an Army Working Population With Nonacute Low Back Pain: A Randomized Controlled Trial. Arch Phys Med Rehabil 2008 July 31 [Epub ahead of print].

22. Van Buuren S, Oudshoorn K. Flexible multivariate imputation by MICE. In: Technical Report TNO Quality of Life. Leiden, The Netherlands, 1999.

23. Altman DG, Schulz KF, Moher D, et al. The revised CONSORT statement for reporting randomized trials: explanation and elaboration. An Intern Med 2001;134:663-94.

24. Boutron I, Moher D, Altman DG, et al. Extending the CONSORT statement to randomized trials of nonpharmalogical treatment: explanation and elaboration. Ann Intern Med 2008;148:295-309.

25. Helmhout PH, Harts CC, Staal JB, de Bie RA. Rationale and design of a multicenter randomized controlled trial on a 'minimal intervention' in Dutch army personnel with nonspecific low back pain [ISRCTN19334317]. BMC Musculoskelet Disord 2004;5:40.

26. Vlaeyen JWS, Kole-Sneijders AMJ, Boeren RGB, Van Eek H. Fear of movement/(re)injury in chronic low LBP and its relation to behavioural performance. Pain 1995;62:363-72.

27. Mayer TG, Gatchel RJ. Functional restoration for spinal disorders: the sports medicine approach. Philadelphia, PA: Lea and Febiger; 1988.

28. Verbunt JA, Seelen HA, Vlaeyen JW, et al. Disuse and deconditioning in chronic low back pain: concepts and hypotheses on contributing mechanisms. EurJPain 2003;7:9-21.

29. Smeets RJ, Wade D, Hidding A, et al. The association of physical deconditioning and chronic low back pain: a hypothesis-oriented systematic review. Disabil Rehabil 2006;28:673-93.

30. Hayden JA, JA, van Tulder MW, Malmivaara AV, et al. Meta-analysis: exercise therapy for nonspecific low back pain. Ann Intern Med 2005;142:765-75.

31. Bronfort $\mathrm{G}$, Goldsmith $\mathrm{CH}$, Nelson $\mathrm{CF}$, et al. Trunk exercise combined with spinal manipulative or NSAID therapy for chronic low back pain: A randomized, observerblinded clinical trial. J Manipulative Physiol Ther 1996;19(9):570-82.

32. Hayden JA, van Tulder MW and Tomlison G (2005b): Systematic review: strategies for using exercise therapy to improve outcomes in chronic low back pain. Annals of Internal Medicine 142: 776-785.

33. ACSM. ACSM position stand on the recommended quantity and quality of exercise for developing and maintaining cardiorespiratory and muscular fitness, and flexibility in adults. Med Sci Sports Exerc 1998;30:975-91. 

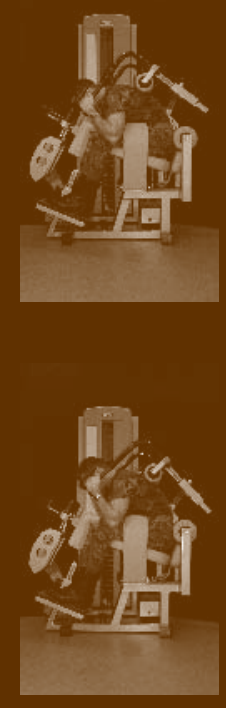

\title{
Summary
}

\author{
Samenvatting
}

Dankwoord

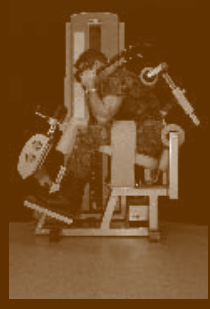

About the author
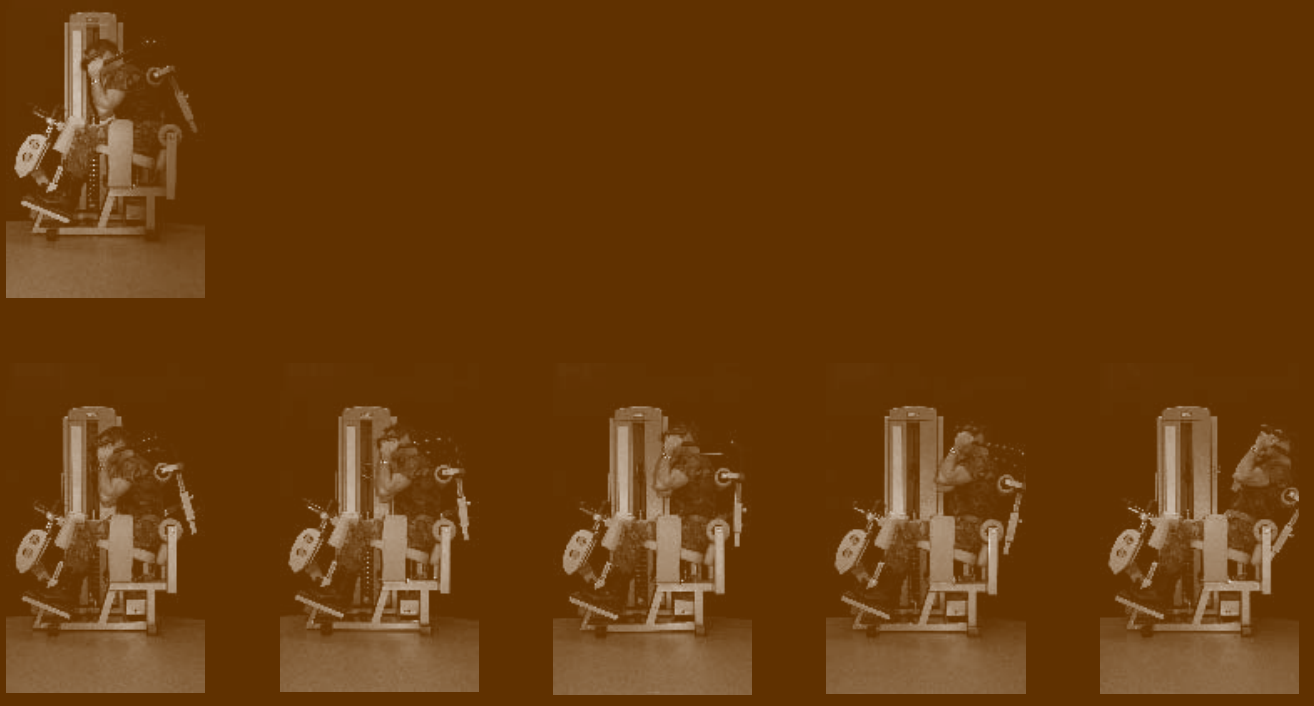


\section{Summary}

This thesis can be divided in three sections, according to the main research questions. In the first section (Chapters 2 to 5 : research question 1), the effectiveness of LES for workers with nonacute nonspecific LBP is studied in three separate randomized controlled trials. The second section (Chapter 6: research question 2) concerns the examination of prognostic factors in the development of LBP in our working population. In the third section (Chapter 7 ) the predictive value of a fatigue test of the isolated lumbar extensors for training progression is evaluated in a young male population without LBP. Moreover, recommendations are given for the design, conduct, and report of clinical trials of exercise therapy in LBP (Chapter 8). For each section, the main study findings are presented below.

\section{Effectiveness of lumbar extensor strengthening}

In the three trials of this thesis, the effectiveness of LES was compared to that of several other 'treatment interventions', i.e., a low-intensity (mobilizing) variant of LES; usual RNLA care (regular physiotherapy mainly consisting of exercise therapy); and a non-treatment (waiting list) group. Effectiveness was primarily measured in terms of improvement in self-assessed functional disability.

In the first trial, the effectiveness of a 12-week high-intensive LES program was compared to a low-intensive program in 81 workers with chronic nonspecific LBP. Our results showed that the two treatment programs led to comparable improvements in all outcome measures, except for mean isometric back strength and fear of movement/(re-)injury score during the follow-up. The high-intensive training group showed a higher strength gain and a smaller decline in fear of movement up to 9 months after treatment, compared to the low-intensity training group. The high-intensive group also showed a somewhat higher treatment compliance and participation rate in post-treatment exercise training. The outcome responses of this study (percentage (non-)response, effect sizes) were comparable with those reported in similar studies with, mostly, more extended exercise programs. 
In the second trial on 65 workers with chronic nonspecific LBP, we compared the effects of an 8-week high-intensive and low-intensive LES training program, respectively, with a waiting list control-group. Most of the comparisons between the exercise groups and the waiting list control-group for global perceived effect, disability, health-related quality of life, fear of movement/(re-)injury and isometric back strength showed point estimates which did not favour the two exercise groups. Immediately after intervention, the high-intensive group reported a greater global perceived effect than the low-intensive group. Quality of life scores were also better in the high-intensive group, but this difference disappeared during follow-up.

In our multicenter trial, we compared a 10-week LES program with regular physiotherapy in the RNLA in a group of 127 workers with nonacute nonspecific LBP. In both intervention arms, main outcomes showed a favorable development over time: short-term effects remained stable or even slightly improved throughout the 1-year follow-up. No significant differences, however, were found between the intervention programs, both in the short and long term.

In summary, all trials showed concordance in their outcomes, in the sense that the LES group showed no clearly favorable results in restoring back function compared to the other study groups.

\section{Prognostic factors for nonacute nonspecific LBP}

In secondary analyses, using a merged dataset of the three trials, we aimed to explore the relative importance of patient factors associated with clinically relevant functional improvements in our overall study population of 273 workers with nonacute nonspecific LBP. Overall, we found four common prognostic factors for improvement directly after treatment as well as 6 months later: functional disability, fear of movement/(re-)injury, psychological distress, and intervention. The factors duration of complaints and supervisor social support were also included in the short-term model, the factors co-worker social support and pain radiation completed the long-term model. The study findings implicate that it can be of use for clinical practitioners to gather pre-treatment information on, particularly, individual levels of functional disability and fear avoidance behaviour. 


\section{Predictive value of back fatigue test}

The purpose of this exploratory study was to determine whether properties of a lumbar fatigue test, using our LES training/testing machine, had predictive value for the amount of progression in a lower back strength-endurance program in a group of 28 untrained young men. We have designed a three-phased fatigue test of the lumbar extensor muscles, consisting of two consecutive measurements of full-range isometric back strength on a lumbar measurement device, separated by a dynamic back extension set to volitional fatigue. Differences between the strength values of the $1^{\text {st }}$ and $3^{\text {rd }}$ step is thought to indirectly reflect individual lumbar muscle fiber composition. We hypothesized that subjects with low levels of fatigue would show a trend towards larger training progression than subjects with high levels of fatigue, as they were expected to be loaded more appropriate to their muscle fiber composition. Based on the results, this hypothesis is rejected. The fatigue indicator in our regression models, i.e., the proportion of strength decline between two successive isometric back strength tests separated by a dynamic set, did not show predictive value in lumbar strength progression. On the other hand, improvement of work capacity during the dynamic part of the baseline fatigue test, had some predictive value. In practice, this means that a lower back training machine could be used at baseline to assist in tailoring individual lumbar training regimes, without the additional use of an isometric-strength testing module.

\section{Recommendations for conducting clinical trials on exercise therapy in LBP} In plenary presentations and discussions of an expert panel that was held at the Amsterdam Forum VIII on Low Back Pain Research in Primary Care, important issues regarding the design, conduct and reporting of exercise trials were highlighted to provide suggestions for improvement in each domain. This led to the following recommendations: (1) Specify a theoretical framework for exercise therapy for designing the intervention and selecting appropriate treatment efficacy measures; (2) To overcome blinding problems in exercise trials, include sham interventions and assess the success of blinding; (3) Evaluate the role of patient-provider interactions through the use of self-report or observational measures; (4) To assure quality of treatment, use exercise interventions that reference existing exercise guidelines; (5) Use subgroup analyses to identify 
subgroups of patients most likely to benefit; (6) In reporting exercise trials, include an extensive description of the study population, a detailed exercise protocol (e.g., mode, intensity, duration, and frequency), and some measure of patient compliance; and (7) Trial registration and publication of detailed study protocols may help to strengthen the evidence base concerning exercise therapy for low back pain. 


\section{Samenvatting}

In dit proefschrift zijn drie delen te onderscheiden. In het eerste deel wordt in drie gerandomiseerde, gecontroleerde studies (trials) de effectiviteit van geïsoleerde lagerugtraining onderzocht in een groep werknemers met niet-acute, aspecifieke lagerugklachten (hoofdstuk 2-5: vraagstelling 1). Het tweede deel behandeld een analyse van prognostische factoren voor een gunstig beloop van de rugklachten op middenlange termijn (hoofdstuk 6 : vraagstelling 2 ). In het derde deel wordt allereerst van een vermoeidheidstest van de lage rug de predictieve waarde voor trainingsprogressie onderzocht in een groep jonge mannen zonder rugklachten (hoofdstuk 7). Daarnaast worden vanuit een expert panel aanbevelingen gegeven voor verbetering van het design, de uitvoering en de rapportage van klinische trials naar oefentherapie bij lagerugklachten (hoofdstuk 8). Voor elk van de drie delen worden hieronder de belangrijkste bevindingen samengevat.

\section{Effectiviteit van geïsoleerde lage-rugtraining}

In de drie trials van dit proefschrift is de effectiviteit van geïsoleerde lage-rugtraining vergeleken met die van andere 'behandelinterventies', namelijk met die van een laagintensieve (mobiliserende) variant van het trainingsprogramma, met reguliere fysiotherapie binnen de Koninklijke Landmacht dat voornamelijk bestaande uit oefentherapie en met een wachtlijstgroep. Effectiviteit is hoofdzakelijk gemeten in termen van (verbetering in) functionele beperkingen als gevolg van de rugklachten.

In de eerste trial is de effectiviteit van een 12 weken durend hoogintensief - want progressief - trainingsprogramma voor de geïsoleerde lage rug afgezet tegen die van een laagintensief programma, bij een groep van 81 werknemers met chronische, aspecifieke lagerugklachten. De resultaten geven aan dat de twee behandelprogramma's tot vergelijkbare verbeteringen op alle uitkomstmaten leiden, behalve voor isometrische rugspierkracht en bewegingsangst als gevolg van de rugklachten. De hoogintensieve groep laat ten opzichte van de laagintensieve groep in 9 maanden follow-up meer progressie in rugspierkracht zien, maar een kleinere afname in bewegingsangst. De hoogintensieve groep 
laat daarnaast een wat grotere therapietrouw zien, evenals een wat hogere deelname aan oefenprogramma's na afloop van de behandelperiode. De uitkomsten van deze studie (percentage non-respons, effect sizes) zijn vergelijkbaar met die van andere gerapporteerde studies naar uitgebreidere vormen van oefentherapie van lagerugklachten.

In de tweede trial is bij 65 werknemers met chronische, aspecifieke lage-rugklachten de effectiviteit vergeleken van een 8 weken durend hoogintensief en laagintensief trainingsprogramma voor de geïsoleerde lage rug ten opzichte van elkaar en ten opzichte van een wachtlijstgroep. De meeste uitkomsten op zelfervaren verandering in klachten, functionele beperkingen, gezondheidsgerelateerde kwaliteit van leven, bewegingsangst en rugspierkracht geven puntschattingen die niet duidelijk in het voordeel van de twee behandelprogramma's spreken. Direct na de interventieperiode scoort de hoogintensieve groep gunstiger op zelfervaren verandering in klachten ten opzichte van de laagintensieve groep. Datzelfde geldt voor de scores op kwaliteit van leven, maar dit verschil verdwijnt na langere follow-up.

In onze multicenter trial hebben we een 10 weken durend trainingsprogramma voor de geïsoleerde lage rug vergeleken met de reguliere oefentherapie binnen de Koninklijk Landmacht bij een groep van 127 werknemers met niet-acute, aspecifieke lage-rugklachten. Beide interventie-armen laten een gunstige ontwikkeling in klachten zien: korte-termijn effecten blijven aanwezig of verbeteren zelfs licht na 1 jaar follow-up. Er worden echter geen significante verschillen gevonden in de resultaten voor beide behandelprogramma's op korte termijn en lange termijn.

Samengevat geven de trials een consistent beeld, namelijk dat een hoogintensief trainingsprogramma voor de geïsoleerde lage rug geen duidelijke meerwaarde heeft boven de andere onderzochte behandelprogramma's.

\section{Prognostische factoren voor niet-acute, aspecifieke lage-rugklachten}

In secundaire analyses van een (vanuit de drie trials samengestelde) database is de prognostische waarde onderzocht van diverse baseline patiëntkarakteris- 
tieken voor klinisch relevante functionele verbeteringen op de korte (direct na behandelperiode) en langere termijn ( $1 / 2$ jaar follow-up), bij een groep van 273 werknemers met niet-acute, aspecifieke lagerugklachten. Op zowel korte als langere termijn zijn de volgende vier prognostische factoren gevonden: functionele beperkingen, bewegingsangst, psychologische distress en behandeltype. De factoren 'duur van de klachten' en 'sociale support van de baas' completeren het korte-termijn model, de factoren 'sociale support collega's' en 'pijnuitstraling' het lange-termijn model. De resultaten van deze analyses impliceren dat informatie vooraf over (vooral) de mate van functionele beperkingen en bewegingsangst bij de patiënt voor de zorgverlener van waarde kan zijn in het behandelplan.

\section{Predictieve waarde vermoeidheidstest voor de lage rug}

In een explorerende deelstudie is bij een groep van 28 ongetrainde jonge mannen onderzocht of een vermoeidheidstest voor de lage rug predictieve waarde heeft voor de mate van progressie in een 8-weeks trainingsprogramma gericht op krachtuithoudingsvermogen van de lage rug. Met behulp van een lagerugmachine waarmee de rugspierkracht geïsoleerd kan worden getraind en gemeten, is een vermoeidheidsprotocol ontwikkeld bestaande uit drie opeenvolgende fasen: twee isometrische-krachtmetingen van de lage rug in de volledige range of motion, met ertussen een dynamische set tot falen. De hypothese was dat deelnemers met een laag vermoeidheidsniveau (krachtsafname tussen 1e en $3 e$ fase) een tendens richting grotere trainingsprogressie zouden laten zien dan deelnemers met een hoog vermoeidheidsniveau, omdat het trainingsprogramma beter zou aansluiten bij hun rugvezeltypering. Op basis van de analyses wordt deze hypothese verworpen. De (procentuele) krachtsafname van de twee isometrische tests voor de lage rug heeft in de regressiemodellen geen predictieve waarde voor trainingsprogressie. De arbeid geleverd in de dynamische set heeft daarentegen wel voorspellende waarde. In de trainingspraktijk zou een lagerugmachine van waarde kunnen zijn in de individualisering van trainingsprogramma's, zonder gebruikmaking van een module dat de isometrische rugspierkracht meet. 


\section{Aanbevelingen voor het uitvoeren van klinische trials naar oefentherapie bij}

\section{lagerugklachten}

In plenaire presentaties en aansluitende discussies van een expert panel tijdens het "Amsterdam Forum VIII on Low Back Pain Research in Primary Care" is een aantal aanbevelingen naar voren gekomen voor het opzetten, uitvoeren en rapporteren van klinische trials naar oefentherapie bij lage-rugklachten, De volgende aanbevelingen zijn geformuleerd: (1) maak op basis van een theoretisch model voor de werkzaamheid van oefentherapie een keuze voor de studie- design en uitkomstmaten; (2) teneinde problemen rondom blindering te voorkómen: gebruik placebo-interventies en evalueer het succes van de blindering; (3) evalueer de rol van interacties tussen patiënt en behandelaar met hulp van zelfrapportage- of observatie-instrumenten; (4) teneinde de behandelkwaliteit te garanderen: gebruik oefentherapetische behandelvormen conform bestaande richtlijnen; (5) pas subgroepanalyses toe om subgroepen van patiënten te identificeren die het meest gebaat zijn bij oefentherapie; (6) zorg in de rapportage van trials over oefentherapie voor een uitgebreide beschrijving van studiepopulatie, trainingsprotocol (bv. type, duur, frequentie en intensiteit) en therapietrouw; (7) trialregistratie en publicatie van een designartikel draagt bij aan de bewijsvoering van oefentherapie bij lagerugklachten. 


\section{Dankwoord}

Hoewel de voorkant van dit proefschrift slechts één naam vermeldt, is alles wat zich tussen de twee kaften bevindt het noeste werk van velen geweest. Graag wil ik die velen op deze plaats danken en eren, een paar mensen in het bijzonder.

Chris (drs. C.C. Harts), als eerste wil ik jou bedanken. Vanaf dag één hebben we dit onderzoek samen getrokken en geduwd. Tot het bittere einde - als paranimf bij de verdediging - heb je me terzijde gestaan. Zonder jou was er geen onderzoek en ook geen promotie geweest; zonder je directe betrokkenheid was het een aanmerkelijk moeizamer traject geworden. Dank voor je verlegenmakende loyaliteit, je professionaliteit en je fijne persoon.

Rob (prof.dr. R.A. de Bie), niemand had dit onderzoek met zo'n schwung kunnen begeleiden als jij dat kunt. Dank dat je het onderzoeksproject zag zitten en deze mogelijkheid voor een externe promotieplaats bij je capaciteitsgroep hebt geboden. Je autoriteit op het gebied van de evidence-based physiotherapy, maar ook je doortastendheid en je humor - 'die jongens van het leger' enzo - maakten het proces vele malen lichter.

Bart (dr. J.B. Staal), toonbeeld van de nuchterheid die bij goede onderzoekers hoort, dank voor die talloze keren dat je met één correctie, een referentie die je ergens vandaan toverde of een opmerking-die-niets-aan-duidelijkheid-te-wensen-overliet, al onze wilde, onhaalbare of tamelijk nutteloze ideeën weer even tot de juiste proporties terugbracht. Dank ook voor het feit dat ik, ondanks de afstand, altijd bij je terecht kon. Ik had me geen betere 'dagelijks begeleider' kunnen wensen.

Frank (kol-arts b.d. F.M. Bertina), Godfather van TGTF en in het bijzonder van het concept van 'geïsoleerde lagerugtraining'. Jouw niet aflatende enthousiasme, nieuwsgierigheid en geloof in de waarde van de sportgeneeskunde in de behandeling van rugklachten vormden het gewapende fundament van dit onderzoek. De eindeloze load curve adjustment discussies en nonmuscular torque paperclipmetingen zijn klassiekers in het onderzoeksrariteitenkabinet van TGTF geworden. 
Jos (drs. M.J. van Dijk), dank voor de ruimte die je me gaf om dit onderzoek naast de overige werkzaamheden tot een goed einde te brengen. Je scherpe visie, heldere analyses en je unieke vermogen om problemen te modelleren hebben een belangrijke bijdrage geleverd aan het uiteindelijke resultaat.

Overige (ex-)collega's van TGTF, dank voor jullie steun en belangstelling gedurende het onderzoekstraject. Ik voel me vereerd deel uit te mogen maken van zo'n geëngageerde club professionals. Een speciaal woord van dank aan Marc voor het ter beschikking stellen van lijf en leden aan de wetenschap (lees: kaft).

René (kol-arts b.d. R. Roelofs) en Kees (kol-arts C. IJzerman), bijzondere dank voor de mogelijkheid die jullie ons vanuit jullie toenmalige functie geboden hebben om dit onderzoek bij TGTF uit te voeren en door te trekken naar een promotietraject. Hartelijk dank ook aan de overige leden van de begeleidingscommissie (prof.dr. H. van Mameren, kol-arts b.d. E. Hendriks) voor de kritische procesbegeleiding gedurende de moeilijke fasen van het onderzoek.

Colette, Irene, Helma, Edward, Frank, Frans, John, Marcel, Paul, Rob en alle andere fysiotherapeuten van de militaire gezondheidscentra die betrokken waren bij de ALBATRoS studie: dank voor jullie inzet en professionaliteit bij de begeleiding van de deelnemers. En ook natuurlijk voor al dat vervelende (rood-witgele) papierwerk dat onvermijdelijk hoort bij het doen van onderzoek.

Hans (dhr. H. de Haas), zonder jouw professionele ondersteuning en creatieve brainwaves had het proefschrift er nooit zo prachtig vormgegeven uit kunnen zien. Dank voor het geleverde monnikenwerk.

Pap en mam, ik wil dit boekje aan jullie opdragen, aan jullie onvoorwaardelijke liefde en zorg. Ik weet hoe jullie hebben meegeleefd en hoe me dit gesteund heeft door alle fasen van de promotie heen. Familie, schoonfamilie en vrienden, dank voor jullie belangstelling en morele ondersteuning. Het is fijn werken als je weet altijd te kunnen terugvallen op de mensen die je liefhebt. Tot slot: Lars, paranimf for life, dank voor al het onbeschrijfbare. 


\section{About the author}

Pieter Helmhout was born on July 8th, 1969 in Rheden, The Netherlands.

After obtaining his secondary school diploma in 1987 at the Christelijk Lyceum Arnhem, he started his study at the Faculty of Health Science of the Katholieke Universiteit Nijmegen. In 1993 he graduated with a major in human movement science with subsidiaries in epidemiology and biostatistics. From September 1993 till July 1994 he attended military service as a reserve-officer with academic degree at the Department of Training Medicine and Training Physiology (TGTF) of the Royal Netherlands Army. He signed on for another year and from September 1995 to date, he has joined the TGTF team as a civil member. As a senior researcher, he is currently working on topics related to military healthrelated fitness, health education \& promotion, and evidence-based medicine. 

